\title{
Adjoint tomography of the Hikurangi subduction zone and the North Island of New Zealand
}

\author{
by \\ Bryant Chow
}

\begin{abstract}
A thesis
submitted to Victoria University of Wellington

in fulfillment of the requirements for the degree of

Doctor of Philosophy in Geophysics
\end{abstract}

School of Geography, Environment and Earth Sciences

Victoria University of Wellington

Wellington, New Zealand

2021 

"The greatest value of a picture is when it forces us to notice what we never expected to see." - John Tukey 



\section{Abstract}

Seismic tomography is a powerful tool for understanding Earth structure. In New Zealand, velocity models derived using ray-based tomography have been used extensively to characterize the complex plate boundary between the Australian and Pacific plates. Advances in computational capabilities now allow us to improve these velocity models using adjoint tomography, an imaging method which minimizes differences between observed and simulated seismic waveforms. We undertake the first application of adjoint tomography in New Zealand to improve a ray-based New Zealand velocity model containing the Hikurangi subduction zone and the North Island of New Zealand.

In support of this work we deployed the Broadband East Coast Network (BEACON), a temporary seismic network aimed at improving coverage of the New Zealand permanent network, along the east coast of the North Island. We concurrently develop an automated, open-source workflow for full-waveform inversion using spectral element and adjoint methods. We employ this tool to assess a candidate velocity model's suitability for adjoint tomography. Using a 3D ray-based traveltime tomography model of New Zealand, we generate synthetic seismic waveforms for more than 10000 source-receiver pairs and evaluate waveform misfits. We subsequently perform synthetic checkerboard inversions with a realistic New Zealand source-receiver distribution. Reasonable systematic time shifts and satisfactory checkerboard resolution in synthetic inversions indicate that the candidate model is appropriate as an initial model for adjoint tomography. This assessment also demonstrates the relative ease of use and reliability of the automated tools.

We then undertake a large-scale adjoint tomography inversion for the North Island of New Zealand using up to 1800 unique source-receiver pairs to fit waveforms with periods 4-30 s, relating to minimum waveform sensitivities on the order of 
$5 \mathrm{~km}$. Overall, 60 geographically well-distributed earthquakes and as many as 88 broadband station locations are included. Using a nonlinear optimization algorithm, we undertake 28 model updates of $\mathrm{V}_{p}$ and $\mathrm{V}_{s}$ over six distinct inversion legs which progressively increase resolution. The total inversion incurred a computational cost of approximately 500000 CPU-hours. The overall time shift between observed and synthetic seismograms is reduced, and updated velocities show as much as $\pm 30 \%$ change with respect to initial values. A formal resolution analysis using point spread tests highlights that velocity changes are strongly resolved onland and directly offshore, at depths above $30 \mathrm{~km}$, with low-amplitude changes (>1\%) observed down to $100 \mathrm{~km}$ depth. The most striking velocity changes coincide with areas related to the active Hikurangi subduction zone.

We interpret the updated velocity model in terms of New Zealand tectonics and geology, and observe good agreement with known basement terranes, and major structural elements such as faults, sedimentary basins, broad-scale subductionrelated features. We recover increased spatial heterogeneity in seismic velocities along the strike of the Hikurangi subduction zone with respect to the initial model. Below the East Coast, we interpret two localized high-velocity anomalies as previously unidentified subducted seamounts. We corroborate this interpretation with other work, and discuss the implications of deeply subducted seamounts on slip behavior along the Hikurangi margin. In the Cook Strait we observe a low-velocity zone that we interpret as a deep sedimentary basin. Strong velocity gradients bounding this low-velocity zone support hypotheses of a structural boundary here separating the North and South Islands of New Zealand. In the central North Island, low-velocity anomalies are linked to surface geology, and we relate seismic velocities at depth to crustal magmatic activity below the Taupō Volcanic Zone.

This new velocity model provides more accurate synthetic seismograms and additional constraints on enigmatic tectonic processes related to the North Island of New Zealand. Both the velocity model itself, and the underpinning methodological contributions, improve our ever-expanding understanding of the North Island of New Zealand, the Hikurangi subduction zone, and the broader AustralianPacific plate boundary. 


\section{Acknowledgments}

I am incredibly grateful to my supervisors Yoshi Kaneko and John Townend, for their guidance, patience, and encouragement. They have been excellent role models, and are the reason my $\mathrm{PhD}$ has been such a positive and fulfilling experience. Thanks to Yoshi for instigating this ambitious project, for setting me off on my current scientific trajectory, and for constantly demonstrating what passionate dedication to good science looks like. Thanks to John for setting a high standard of quality in my research, and always making sure I was enjoying the finer days. I am thankful to my colleagues Carl Tape and Ryan Modrak for helping me understand the intricacies of tomography, and reminding me that what we do is exciting and important.

I am grateful to all those who have supported my work. Thank you to Donna Eberhart-Phillips for her seminal contributions to tomography; without the New Zealand Wide seismic velocity model this work would not have been possible. I acknowledge GeoNet for providing seismic data, because without data there is no tomography. Similarly I thank Stephen Bannister, and the SAHKE team, for additional waveform data. I am grateful to the New Zealand eScience Infrastructure for helping me simulate many thousands of earthquakes on their cluster Māui, and to Alex Pletzer for helping us optimize our codes there. During the interpretation phase of my work, I had enlightening conversations regarding tectonics, geology, and the power of hand-waving with: Calum Chamberlain, Finn Illsley-Kemp, Stephen Bannister, Nick Mortimer, Carolyn Boulton, Laura Wallace, Susan Ellis, Phil Barnes, Tim Little, and Carl Tape. Thanks to Calum for always answering my Python-related questions, and to Lauren Vargo, Hubert Zal, and Katie Woods for proofreading parts of this thesis. Thank you to my thesis committee, Martha Savage, Dan Bassett, and Qinya Liu, for providing thorough, critical feedback on this thesis, and for the insightful discussion during my defense. 
I would like to acknowledge everyone that assisted with the Broadband East Coast Network (BEACON). In particular: Yoshi, Jonathan Hanson, and Rory Hart, who each helped make me a more competent field scientist. Thanks also to Hubert Zal, Dani Lindsay, Jesse Kearse, and Conrad Burton for joining me in lugging batteries around Hawke's Bay. Thank you to Bill Fry and Emily Warren-Smith for lending us some of those batteries to lug around. I would like to express gratitude to all the land owners who allowed us to set up seismometers on their property, and who graciously welcomed us back each servicing trip.

I am grateful for the financial support I've received. Thank you to the Royal Society of New Zealand Te Apārangi for funding this work, and the New Zealand Ministry of Business, Innovation and Employment for additional funding. Thank you to the Geoscience Society of New Zealand for support through the Jim Ansell Geophysics Scholarship. Using their Young Researcher Travel Grant, I was able to spend a lovely week in Corsica learning about wave physics.

To all the friends in Cotton building that have made this a wonderful experience: Lauren, Hubert, Chet, Kostas, Katie, Dani, Kenny, Dom, Abhi, Calum, Jamey, Katelyn, Jesse, Weiwei, Andrea, Finn, Jürgen, Marcel, Matt, Marjo, Alanna - you all rock. I'm grateful to have been friends with many of the other SGEES postgrads and staff, who made this department feel like a family. Thanks to my office mates past and present: Kostas, Dom, Pasan, Abhi, Hamish, Levan, and Laura. To my flatmates on Atlanta Grove: Matt, Kristyn, Angie, Colin, Tom, Gabriel, Eru thanks for making home a nice place.

Finally to my family: my parents Monica and Steven, and my sister Yvonne - you are the constant in my life, and with your support I feel like I can achieve whatever I put my mind to. Thank you for everything. 


\section{Contents}

Abstract

Acknowledgments vii

Contents ix

List of figures $\ldots \ldots \ldots \ldots \ldots \ldots \ldots \ldots \ldots$

List of tables .....................

1 Introduction 1

1.1 Motivation and objectives ................ 1

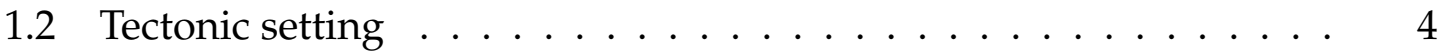

1.3 Seismic tomography . . . . . . . . . . . . . . . . . 10

1.3.1 Ray tomography ................. 12

1.3.2 Finite-frequency tomography ............. 12

1.3.3 Full-waveform tomography ............. 13

1.4 Tomography in New Zealand . . . . . . . . . . . . . . . . 15

1.5 Thesis structure $\ldots \ldots \ldots \ldots \ldots \ldots$

2 Methods and data 19

2.1 Methods ........................ 19

2.1.1 The forward problem ............. 20 
2.1.2 The spectral element method . . . . . . . . . . . 21

2.1 .3 Meshing ....................... 22

2.1.4 The inverse problem .................. 24

2.1.5 The adjoint-state method ............ 25

2.1.6 The L-BFGS algorithm . . . . . . . . . . . . . . 27

2.1.7 Regularization .................. 29

2.2 Data . . . . . . . . . . . . . . . . . . . 29

2.2 .1 Study area . . . . . . . . . . . . . . . . 29

2.2.2 Earthquake sources ................ 30

2.2 .3 Receivers....................... 31

2.3 The Broadband East Coast Network (BEACON) . . . . . . . . 32

2.3.1 Deployment .................... 34

2.3.2 Data availability ................. 39

2.3 .3 Instrument response $\ldots \ldots \ldots \ldots$

2.3.4 Long-period data issues . . . . . . . . . . . . 40

3 An automated workflow for adjoint tomography 47

3.1 Introduction ....................... 48

3.2 Misfit assessment of an initial 3D velocity model . . . . . . . . 51

3.2.1 Simulation framework . . . . . . . . . . 52

3.2.2 Misfit assessment . . . . . . . . . . . . . 5 54

3.3 Automated inversion workflow . . . . . . . . . . . 58

3.3 .1 SeisFlows .................... 61

3.3 .2 Pyatoa ............................. 62

3.4 Synthetic inversions . . . . . . . . . . . . . 66 
3.4.1 Inversion setup . . . . . . . . . . . . . 66

3.4 .2 Computational expense ............... 68

3.4.3 Resolution and parameter testing . . . . . . . . . 69

3.5 Discussion ......................... 74

3.5.1 Choice of workflow tools ............. 74

3.5.2 Potential applications of an automated inversion workflow $\quad 74$

3.5.3 Towards a full-scale North Island inversion . . . . . . . . . 75

3.6 Conclusions ...................... 76

4 Adjoint tomography of the North Island of New Zealand 79

4.1 Introduction . . . . . . . . . . . . . . . 80

4.2 Data .................................. 81

4.2.1 Study area and starting model . . . . . . . . . . . 81

4.2 .2 Earthquake sources . . . . . . . . . . . . 82

4.2 .3 Receivers .................... 83

4.3 Methods ............................. 85

4.3.1 Forward problem, meshing, misfit quantification . . . . 85

4.3.2 Inverse problem, nonlinear optimization, regularization . . 87

4.3 .3 Inversion legs . . . . . . . . . . . . 88

4.4 Inversion results . . . . . . . . . . . . . . . . . 90

4.4.1 Velocity changes ................ 91

4.4.2 Waveform improvement .............. 96

4.4 .3 Bulk misfit assessment . . . . . . . . . . . . 98

4.4 .4 Computational expense . . . . . . . . . . . . 102

4.5 Resolution analysis . . . . . . . . . . . . . . . . 102 
4.5.1 Point spread function volumes . . . . . . . . . . . 103

4.5.2 Point-localized perturbations ............. 104

4.6 Conclusions ........................ 106

5 Tectonic and geologic interpretations of an adjoint tomography velocity model of the North Island of New Zealand

5.1 Introduction . . . . . . . . . . . . . . . . 108

5.2 Discussion ............................. 108

5.2.1 Comparisons with geology and tectonics . . . . . . . . 108

5.2.2 Along-strike crustal heterogeneity . . . . . . . . . . 113

5.2.3 East Coast high-velocity anomalies . . . . . . . . . . 115

5.2.4 Cook Strait velocity gradient . . . . . . . . . . . . . . 121

5.2.5 Taupō Volcanic Zone velocity anomalies . . . . . . . . . . . 125

5.2.6 Implications of strong velocity changes . . . . . . . . . 129

5.3 Conclusions ....................... 130

6 Synthesis 133

6.1 Revisiting research objectives ................ 134

6.2 Potential for future work . . . . . . . . . . . . . . . . 136

6.2.1 A New Zealand adjoint tomography model . . . . . . . . . . 136

6.2.2 Targeted geophysical studies ............. 138

6.2.3 Earthquake relocation ............... 141

6.3 Concluding statement $\ldots \ldots \ldots \ldots \ldots$. . . . . . . . . . . . . .

Appendices 143

A Windowing parameters ................... 143

B Moment tensor preprocessing . . . . . . . . . . . . . 145 
C Supplementary material for Chapter $2 \ldots \ldots . . . . . . . .147$

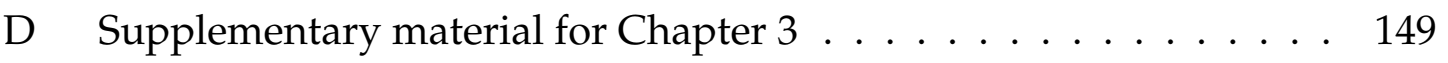

E Supplementary material for Chapter $4 \ldots \ldots$. . . . . . . 155

F Inversion parameter tables . . . . . . . . . . . . . . . 159

Bibliography 167 


\section{List of Figures}

1.1 Tectonic setting of the North Island, New Zealand . . . . . . . . . 5

1.2 New Zealand geology $\ldots \ldots \ldots \ldots$ 7 . . . . . . . .

1.3 Geodetic observations of the Hikurangi subduction zone . . . . . 9 9

1.4 The finite-frequency banana-doughnut paradox $\ldots \ldots \ldots$

2.1 An example of spectral convergence ............ 22

2.2 1D spectral element decomposition example . . . . . . . . 23

2.3 Hexahedral spectral element mesh chunks . . . . . . . . . 24

2.4 Construction of an event sensitivity kernel through adjoint methods 28

2.5 Gradient smoothing through convolution with a 3D Gaussian . . . 30

2.6 Station map of permanent and temporary networks used in inversion 33

2.7 BEACON station locations . . . . . . . . . . . . . 36

2.8 Example BEACON seismometer installation . . . . . . . . 38

2.9 BEACON operations timeline . . . . . . . . . . . . 41

2.10 Power spectral densities for BEACON stations . . . . . . . . . 42

2.11 BEACON long-period data issue . . . . . . . . . 45

2.12 BEACON and GeoNet amplitude ratios for teleseismic earthquakes 46

3.1 Source-receiver map for synthetic inversions . . . . . . . . . . 50

3.2 Initial velocity model (M00) in $\mathrm{V}_{p}$ and $\mathrm{V}_{s} \ldots \ldots \ldots$ 
3.3 Synthetic waveforms using a ray-based tomography model . . . . . 56

3.4 Misfit histograms assessing a ray-based tomography model . . . . 59

3.5 Diagrammatic adjoint tomography workflow . . . . . . . . . . 60

3.6 Pyatoa example, misfit assessment for one source-receiver pair . . 64

3.7 Synthetic inversion summary figure . . . . . . . . . . . 67 67

3.8 Synthetic inversion convergence behavior . . . . . . . . . 71

3.9 Misfit histograms for synthetic inversions . . . . . . . . . . . 72

3.10 Synthetic inversion waveform improvement $\ldots \ldots \ldots$

3.11 Available data for full-scale North Island inversion . . . . . . . . . . 77

4.1 Inversion source-receiver map . . . . . . . . . . . . . . 84

4.2 Coarse and fine mesh resolution comparison . . . . . . . . 86

4.3 Full-scale inversion convergence plot . . . . . . . . . . . . . . 90

4.4 $\mathrm{V}_{s}$ comparisons of initial (M00) and final (M28) velocity models . . 92

4.5 Select $V_{s}$ net model updates at $5 \mathrm{~km}$ depth . . . . . . . . . . 94

4.6 Select $V_{p}$ net model updates at $5 \mathrm{~km}$ depth $\ldots \ldots$

4.7 Comparison of initial and final $\mathrm{V}_{p} / \mathrm{V}_{s}$ models at $5 \mathrm{~km}$ depth . . . 97

4.8 Representative waveform improvement figures . . . . . . . . . . 99

4.9 Inversion and post-hoc misfit histograms . . . . . . . . . . . . 101

$4.10 \mathrm{~V}_{S S}$ point spread function volumes . . . . . . . . . 105

5.1 Velocity model comparison with New Zealand geology . . . . . . . 109

5.2 Comparisons with geologic cross sections . . . . . . . . . . 112

5.3 Along-strike heterogeneities in $\mathrm{V}_{p} / \mathrm{V}_{s} \ldots \ldots \ldots \ldots$

5.4 East Coast high-velocity anomalies and point spread tests . . . . . 117

5.5 Evidence for deeply subducted seamounts below the East Coast . . 119 
5.6 Cook Strait velocity gradient $\ldots \ldots \ldots$. . . . . . . . . . . . .

5.7 Potential tectonic origin of Cook Strait velocity gradient . . . . . . . 124

5.8 Taupō Volcanic Zone velocity anomalies . . . . . . . . . . . . . . 127

6.1 An extended North Island mesh . . . . . . . . . . . . . . . . 139

6.2 Available data for a South Island inversion . . . . . . . . . . . . . . 140

C.1 Select PPSD comparisons for BEACON and GeoNet short-periods . 148

D.1 3D vs.1D model synthetic seismogram comparison . . . . . . . . 150

D.2 1D velocity model misfit histograms . . . . . . . . . . . . 151

D.3 Radial and transverse data-synthetic comparisons . . . . . . . . 152

D.4 Pyatoa + SeisFlows workflow flowchart . . . . . . . . . . . 153

D.5 Cross-correlation vs. multitaper misfit synthetic convergence plot . 154

E.1 $\mathrm{V}_{p}$ comparisons of initial (M00) and final (M28) velocity models . . 156

E.2 $\mathrm{V}_{p} / \mathrm{V}_{s}$ comparisons of initial (M00) and final (M28) velocity models 157

E.3 $\mathrm{V}_{P P}$ point spread function volumes . . . . . . . . 158 


\section{List of Tables}

2.1 BEACON station information $\ldots \ldots \ldots \ldots \ldots$

2.2 BEACON response file parameters $\ldots \ldots \ldots \ldots \ldots$

A.1 Pyflex windowing parameters . . . . . . . . . . . . . 144

F.1 Overview of inversion leg parameters and results . . . . . . 160

F.2 Descriptions of 60 earthquakes used in inversion . . . . . . . 162

F.3 Descriptions of 60 earthquakes used in post-hoc analysis . . . . 164

F.4 GeoNet stations used in inversion $\ldots \ldots \ldots \ldots$

F.5 Temporary network stations used in inversion . . . . . . . 166 


\section{Chapter 1}

\section{Introduction}

\subsection{Motivation and objectives}

Like taking an X-ray of the Earth, seismic imaging, or tomography, is one of the best tools for illuminating the ground beneath our feet. This knowledge is important for many areas of Earth sciences, including geophysics, geochemistry, and geology, which require accurate descriptions of material properties and structure within the Earth. By improving understanding of Earth structure, seismic tomography not only refines fundamental understanding of the planet's interior, but also derives deeper insights into the driving forces behind natural disasters at its surface, like earthquakes, tsunamis, and volcanic eruptions.

Since the 1950s, seismologists have been devising models of Earth structure based on seismic arrival times (e.g. Bullen, 1950; Gutenberg, 1951). By the 1970s, global seismic networks and the advent of the modern computer allowed us to make use of larger datasets to create more accurate models (e.g. Aki and Lee, 1976; Woodhouse and Dziewonski, 1984; Ekström and Dziewonski, 1998). In step with, and often driving, advancements in computational power and complexity of numerical techniques, the modern practice of seismic tomography (Aki Christofferson and Husebye, 1977, Dziewonski et al., 1977) has produced depictions of the Earth at large scales. These models illuminated sections of Earth's interior, including the lithosphere (Ekström and Dziewonski, 1998: Simons and van der Hilst, 2003), mantle (e.g. Romanowicz, 1991; Zhang and Tanimoto, 1993; Li and Romanowicz, 1996: van der Hilst and Kárason, 1999), ascending mantle plumes (e.g. Bijwaard 
and Spakman, 1999; Ritsema et al., 1999; Su et al., 1992), descending subducted slabs (e.g. Bijwaard et al., 1998; Kárason and Van Der Hilst, 2000; Fukao et al. 2001), and continental roots (Ekstrom et al., 1997; Gung et al., 2003). Since the early 2000s, the progressing development of supercomputers and advanced imaging techniques have allowed for exploitation of more of the seismic wavefield. Yet again these advancements allow us to throw more data and bigger computers at the task of understanding Earth structure, at ever higher resolutions, accuracy, and ever smaller scales (e.g. Chen et al., 2007a; Fichtner et al., 2009; Tape et al., 2010; Zhu et al., 2015; Krischer et al., 2015a; Tao et al., 2018).

In New Zealand, ray-based seismic tomography has produced detailed images of an active convergent plate boundary (Eberhart-Phillips et al., 2005; EberhartPhillips and Reyners, 2012; Eberhart-Phillips and Bannister, 2015; Eberhart-Phillips et al., 2020a). These tomographic models have been used extensively in characterizing the structure, material properties, and slip behavior of the Hikurangi subduction zone (e.g. Reyners et al., 2017; Williams and Wallace, 2018; Henrys et al., 2020). They have similarly improved understanding of the potential for large, megathrust earthquakes, which pose significant risk to New Zealand and surrounding regions (e.g. Eberhart-Phillips and Reyners, 1999; Darby and Beavan, 2001; Nicol and Beavan, 2003; Wallace et al., 2004; Douglas et al., 2005; Eberhart-Phillips et al. 2005: Cochran et al., 2006; Henrys et al., 2006; Reyners et al., 2006; Wallace and Beavan, 2006; Litchfield et al., 2007; Barker et al., 2009; Wallace et al., 2009; Fagereng and Ellis, 2009; Wallace et al., 2014; Kaneko et al., 2018). Despite their wide utility, these models are derived using the simplifying ray theory, where seismic waves are approximated as 2D geometrical rays (Stein and Wysession, 2009). This has been shown to lead to ambiguous interpretations of tectonic features (Section 1.3.1; Marquering et al., 1999; Zhao et al., 2000; Dahlen et al., 2000; Hung et al., 2001; Dahlen and Baig, 2002).

Adjoint tomography is a method for imaging structure which 1) simulates seismic waves by solving the (an)elastic wave equation (Tromp et al., 2005; Fichtner et al. 2006a b; Tape et al., 2007), 2) iteratively improves numerical models using the adjoint-state method (Section 2.1.5, Tarantola, 1984), and 3) in seismology, has historically focused efforts on inverting for high-frequency $(f<0.5 \mathrm{~Hz})$ earthquakegenerated surface waves (e.g. Tape et al., 2010; Fichtner et al., 2010; Krischer et al., 2015a; Zhu et al., 2015; Tao et al., 2018). By solving the seismic wave equation, 
adjoint tomography honors the intrinsic physics of wave propagation, overcoming limitations inherent in ray theory (Section 1.3.2, e.g Montelli et al., 2004a). The transition to such "full-waveform" approaches has been accelerated by the advent of modern supercomputers, and the development of numerical methods with which to accurately simulate seismic wave propagation at a wide range of scales (e.g. Komatitsch et al. 2002). Despite increased accuracy, the computational complexity of adjoint tomography has limited its applicability to long-wavelength crustal and mantle structure (e.g. Fichtner et al., 2010; Krischer et al., 2015a; Zhu et al., 2015; Bozdağ et al., 2016; Tao et al., 2018), or regional settings with sufficient seismicity, and network coverage (e.g. Tape et al. 2010). New Zealand, characterized by high levels of seismicity and a long-standing national seismic network, is one such regional candidate.

In this thesis we leverage modern computational power and numerical techniques to develop and interpret a high-resolution velocity model of the North Island of New Zealand using adjoint tomography (Section 4). This is the first application of adjoint tomography to the New Zealand region. Application of adjoint tomography to other regions has resulted in large velocity changes with respect to initial models, and increased resolution of crustal scale features. For example, Tape et al. (2010) observe $\pm 30 \%$ velocity changes with respect to an initial southern California ray-based traveltime tomography model. To facilitate this research, we deploy a temporary seismic network to improve data coverage in our imaging efforts (Section 2.3), and develop a set of procedures and automated tools to perform adjoint tomography in New Zealand and in other settings (Section 3.3). A primary focus of this thesis is to understand material heterogeneities along the complex Hikurangi subduction margin, as well as its associated tectonic features, and its potential for megathrust slip behavior (Section 5).

In pursuit of these overarching goals, we address the following research objectives:

1. What are the best practices for performing adjoint tomography in New Zealand?

2. How does the application of adjoint methods improve the current ray-based tomography model of New Zealand?

3. Do the resulting variations in seismic velocities show similar changes to other applications of adjoint tomography? 
4. What velocity anomalies are revealed that were not resolvable with previous models and what are the physical interpretations of such anomalies?

5. What are the structural differences, manifest as seismic velocities, between the northern and southern Hikurangi margins, and what are their physical interpretations?

This thesis was conducted under the auspices of a Rutherford Discovery Fellowship, entitled 'Structural controls on earthquake behaviour in the Hikurangi subduction mega-thrust (PI: Yoshihiro Kaneko)', awarded by the Royal Society of New Zealand Te Apārangi. This larger project aims to understand the variation in slip behavior along the Hikurangi subduction interface, and consequently funded the deployment of the temporary seismic network BEACON, described in Section 2.3

\subsection{Tectonic setting}

The Hikurangi subduction zone is a convergent plate boundary at which the Pacific plate subducts westward beneath the Australian plate at a rate of $\sim 40 \mathrm{~mm}$ $\mathrm{yr}^{-1}$ (Figure 1.1; Lewis and Pettinga, 1993; DeMets et al., 1994; Collot et al., 1996; Nicol et al., 2007; Barnes et al., 2010). This region presents itself as a unique tectonic setting in which there exists drastic along-strike differences in structure and interseismic coupling (DeMets et al., 1994; Wallace et al., 2004, 2009; Barnes et al. 2010). Subduction terminates below the northern South Island, where plate convergence becomes dominantly strike-slip along the Alpine Fault (Berryman et al. 1992; Leitner et al., 2001; Norris et al., 2007; Sutherland et al., 2007; Wallace et al. 2007), after a complex transition from subduction to transpression in the Cook Strait and through the Marlborough Fault System (Walcott, 1978; Little and Jones, 1998; Barnes et al., 1998; Eberhart-Phillips and Bannister, 2010). The tail-end of the Tonga-Kermadec-Hikurangi extensional back-arc system terminates below the central North Island in the Taupō Volcanic Zone (TVZ; Wilson et al., 1995, 2009; Barker et al., 2020). Geologically, New Zealand is commonly separated into lithologically distinct basement terranes, overprinted by more modern tectonic processes (Figure 1.2, Mortimer, 2004).

The Hikurangi margin exhibits varying differences in along-strike properties (Wal- 


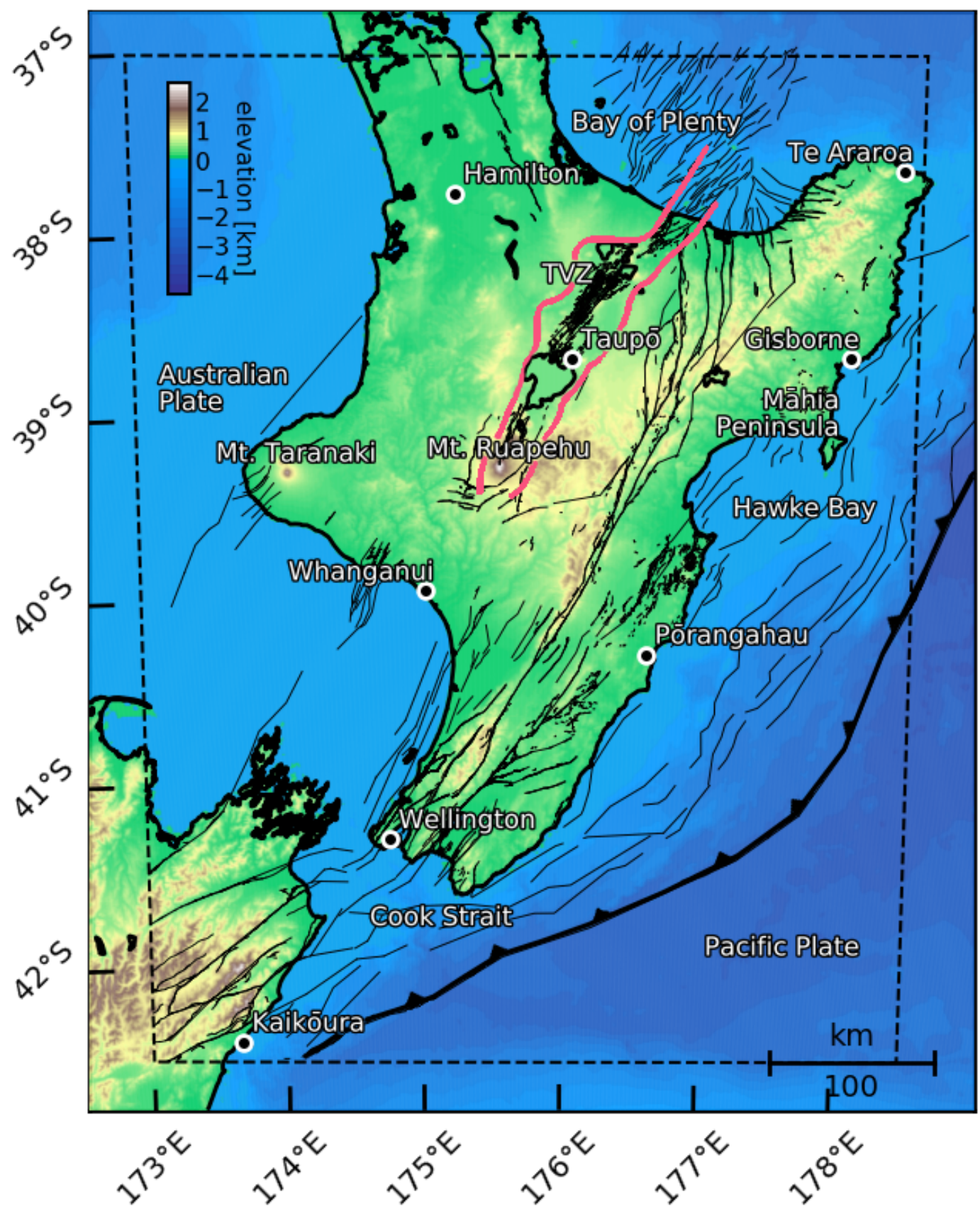

Figure 1.1: Tectonic setting of the North Island, New Zealand. New Zealand active onshore and offshore faults plotted as thin black lines (Litchfield et al. 2014). Color represents elevation values defined by SRTM-30P (Becker et al. 2009). The same elevation values are used to define topography and bathymetry of the numerical mesh (Section 2.1.3). Geographic and tectonic landmarks labelled, with select cities marked by black circles. The solid red lines represent the approximate location of the Taupō Volcanic Zone (TVZ). The tomographic simulation domain is shown by the dashed black line. 
lace et al., 2009). In the northern section of the margin (latitude $<40^{\circ} \mathrm{S}$ ), plate convergence is trench-normal at a rate of roughly $50 \mathrm{~mm} \mathrm{yr}^{-1}$ (Wallace et al., 2009). At the northern Hikurangi margin, the Pacific plate is characterized by the Hikurangi Plateau, a Cretaceous large igneous province (Mortimer and Parkinson, 1996: Taylor, 2006) that is considerably thicker than the subducting oceanic crust further north (Davy et al., 2008; Mochizuki et al., 2019), and dotted with offshore seamounts (Bell et al., 2010; Barnes et al., 2010). Offshore sediment cover and accretionary wedge characteristics vary considerably along-strike as well (Barnes et al. 2010). In the North, sedimentary cover is less than $1 \mathrm{~km}$ thick, and the accretionary wedge is narrow at $\sim 20 \mathrm{~km}$ width. Further south, sedimentary cover thickens to around $5 \mathrm{~km}$ and the accretionary wedge is more well-developed, extending 30$70 \mathrm{~km}$ in width. South of $\sim 40^{\circ} \mathrm{S}$, convergence becomes oblique at more than $40^{\circ}$ to the trench, with a convergence rate of less than $40 \mathrm{~mm} \mathrm{yr}^{-1}$ (Wallace et al. 2004).

Due to buoyancy of the subducting plate, much of the Hikurangi forearc above the shallow part of the plate interface is exposed sub-aerially (Litchfield et al., 2007; Nicol et al., 2007). The Hikurangi trench is consequently nearer to the coastline than in most other well-studied subduction settings, at distances between 40 and $120 \mathrm{~km}$ (Figure 1.1). The plate interface below land is located at $\sim 15 \mathrm{~km}$ depth below the east coast of the North Island (Figure 1.3; Williams et al., 2013). At the southern margin the Pacific plate is characterized by the thick $(\sim 23-26 \mathrm{~km})$ continental crust of the Chatham Rise (Eberhart-Phillips and Reyners, 1997; Reyners et al. 2017). In the Cook Strait and Marlborough Fault System, the plate boundary undergoes a complex transition from oblique subduction to oblique transpression (Pondard and Barnes, 2010; Reyners et al., 2017).

In the North Island, a system of dextral strike-slip faults runs along the eastern edge of the island, thought to accommodate $\sim 6 \mathrm{~mm} \mathrm{yr}^{-1}$ of the total convergence in the north, to $\sim 20 \mathrm{~mm} \mathrm{yr}^{-1}$ in the south (Van Dissen and Berryman, 1996; Beanland, 1995: Beanland and Haines, 1998; Nicol and Beavan, 2003). Further west, in the central North Island, back-arc extension occurs in the Taupō Volcanic Zone (TVZ; Figure 1.1: Wilson et al., 1995, 2009), characterized by high heat flow and geothermal activity, extensional faulting, and corresponding seismicity. The maximum rate of extension in the TVZ is $20 \mathrm{~mm} \mathrm{yr}^{-1}$ (Villamor and Berryman, 2006). Tomographic images of this region show a low-velocity, highly attenuating crust and mantle (Eberhart-Phillips et al., 2020a), while residual gravity maps show low- 


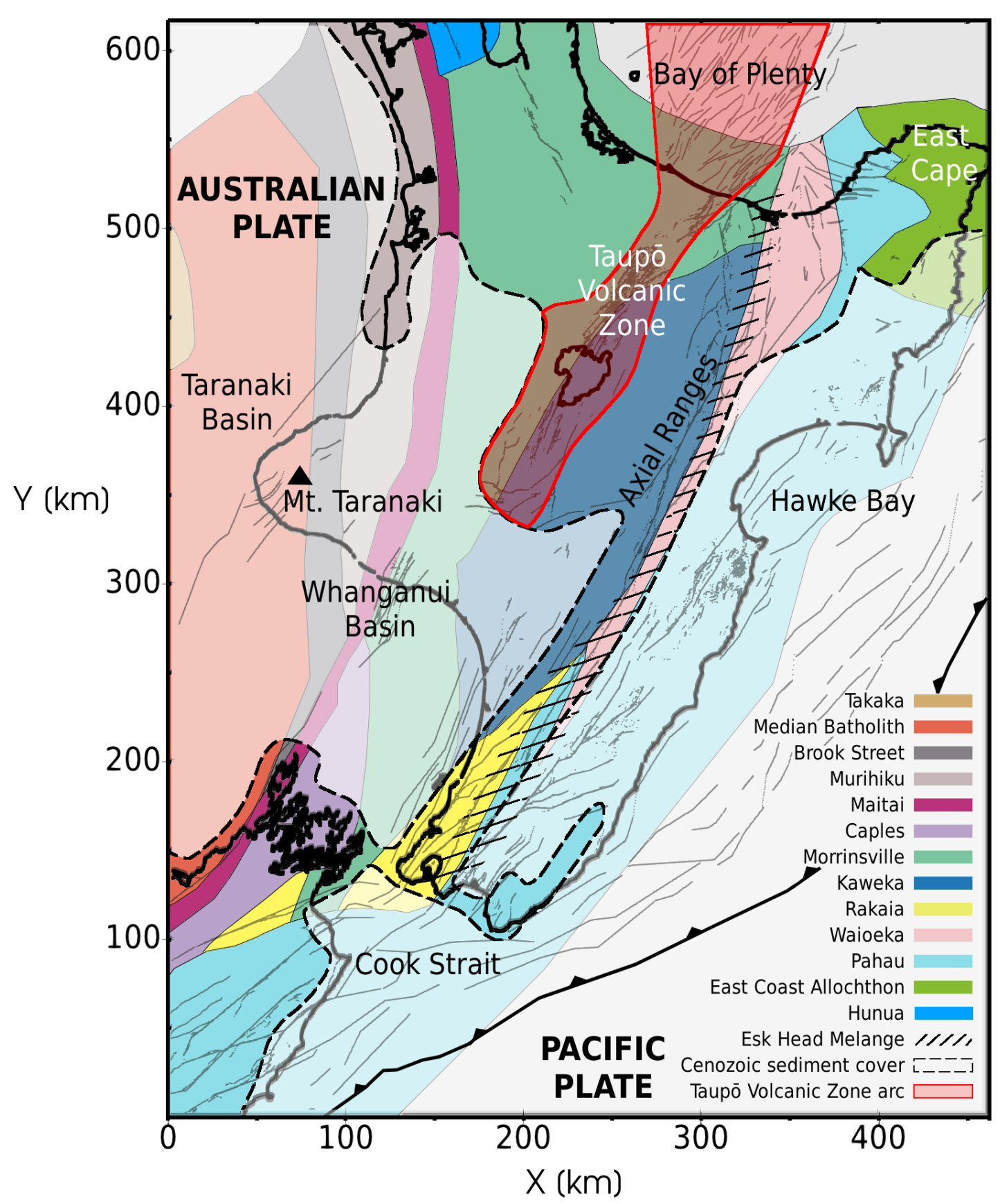

Figure 1.2: New Zealand geology after Mortimer (2004); Edbrooke et al. 2015), shown as basement terranes (solid colors) overlain by volcanic and sedimentary cover (transparent masks). Active onshore and offshore faults from Litchfield et al. (2014). 
density volcanic infill of ancient calderas throughout the region (Stagpoole et al. 2020), as well as a roughly triangular shaped high gravity anomaly bounding this rift zone, referred to as the Central Volcanic Region (CVR; Stern, 1985; Cole et al. 1995). Further west, volcanism is also seen at Mt. Taranaki (Figure 1.1: Neall et al. 1986), which has been noted as unusual in both its location and eruptive composition (Price et al., 1999; Sherburn and White, 2006; Sherburn et al., 2006). North and south of Mt. Taranaki are two large onshore-offshore sedimentary basins, the Taranaki basin (e.g. King and Thrasher, 1996) and Whanganui basin (Figure 1.2 e.g. Carter and Naish, 1998).

Combined seismic and geodetic inversions have been used to determine slip rate deficit along the plate interface (Figure 1.3; Wallace et al., 2012b; Wallace, 2020). These results show that the southern Hikurangi margin is geodetically locked, while the northern portion is freely slipping; the transition between the two styles of slip occurs in the southern Hawke's Bay region (Figure 1.3 B). Geodetic inversions reveal slow slip events (SSEs) in the northern margin occurring at depths of 5-15 km (Figure 1.3A; Wallace, 2020). In contrast, the southern region of the Hikurangi margin is locked to roughly $30 \mathrm{~km}$ depth and experiences SSEs at depths of 30-45 km (Figure 1.3B). Anomalously, a relatively shallow $(<15 \mathrm{~km})$ slow slip event has been observed south of Pōrangahau, within the inferred locked portion of the plate interface (Figure 1.3 $\mathrm{B}$; Wallace et al. 2012b). Based on their findings, Wallace et al. (2009) propose that the geodetically locked southern portion of the margin may be capable of producing a megathrust event as large as $\mathrm{M}_{\mathrm{w}} \sim 8.2-8.7$.

Seismic activity associated with the Hikurangi subduction zone is frequent and varied in terms of faulting mechanism and location (Ristau, 2008; Townend et al. 2012). Subduction seismicity is characterized by intraplate events in the subducting Pacific plate, and interplate seismicity along the principle décollemont. In the upper plate, seismicity is observed in extensional faulting of the central North Island (Darby et al. 2000; Villamor et al., 2017), and dextral strike-slip faulting along the length of the margin (Nicol and Beavan, 2003). In the northern South Island, the $2016 \mathrm{M}_{\mathrm{w}} 7.8$ Kaikoura earthquake produced one of the most complex multifault rupture patterns observed (Hamling et al., 2017; Holden et al., 2017), with an extensive aftershock sequence providing dense seismicity throughout this region. To the north, the $1947 \mathrm{M}_{\mathrm{w}} 7.0$ Gisborne tsunami earthquake generated one of the largest tsunamis in New Zealand history (Bell et al., 2014). 

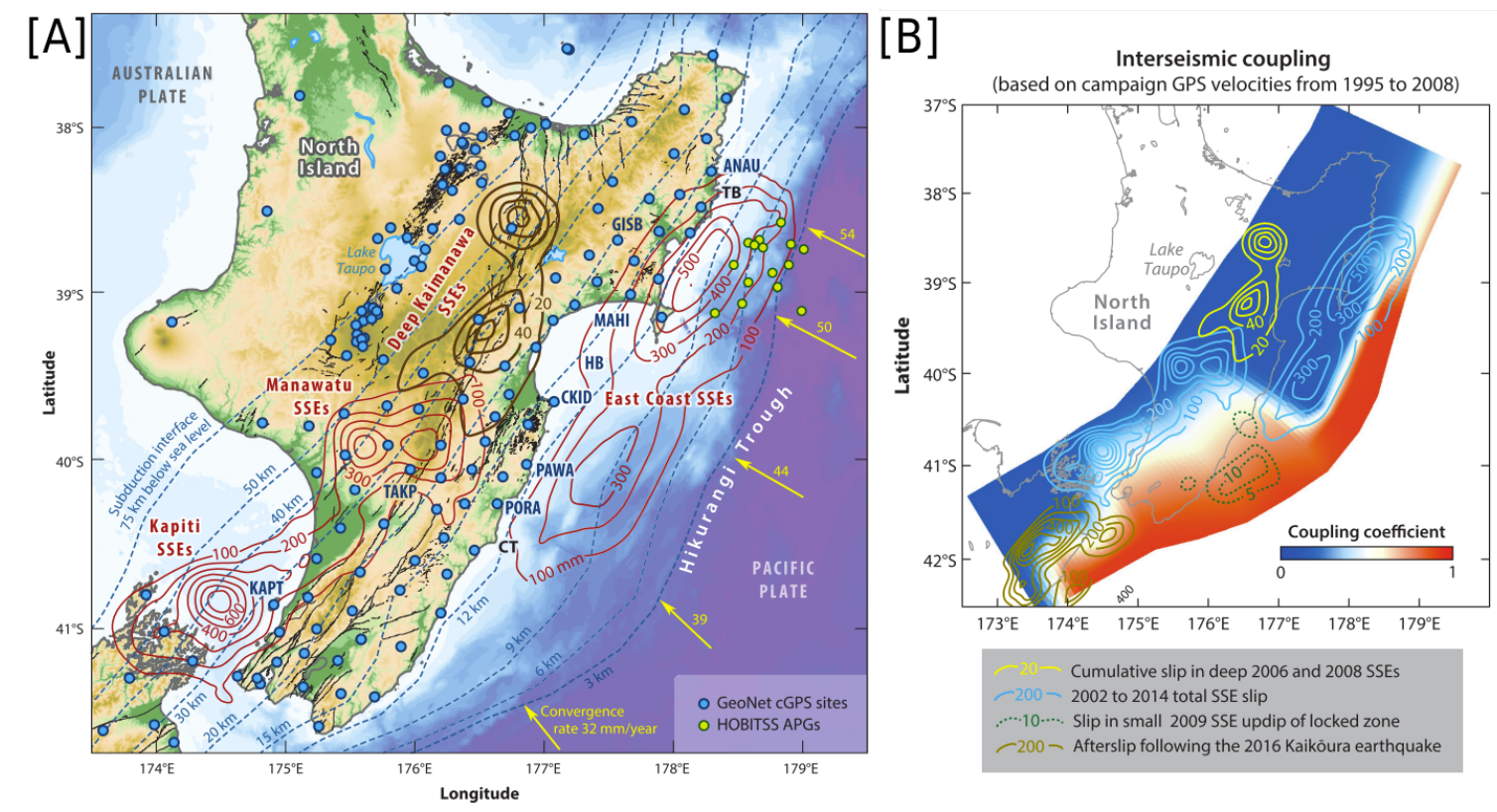

Figure 1.3: An overview of geodetic observations at the Hikurangi subduction zone, after Wallace (2020). A) Cumulative slow slip between 2002 and 2014. Red contours show large (100 mm contours) slow slip events, while brown contours show small $(20 \mathrm{~mm}$ contours), deep SSEs. Blue dots represent GeoNet continuous GPS sites. Green dots show absolute pressure gauges deployed between 2014 and 2015. Plate interface model shown as dashed lines from Williams et al. (2013). B) Interseismic coupling based on campaign GPS from 1995-2008. Colors show plate coupling coefficient, or the ratio of long-term averaged slip rate over short-term creep rate. A value of 0 corresponds to a freely slipping interface, while 1 corresponds to a region of locked coupling. 
Seismic tomography has been an excellent tool for trying to understand and interpret the along-strike variations of slip-behavior observed on the Hikurangi interface. Variations in pore fluid pressure (King Hubbert and Rubey, 1959), temperature (Sibson, 1982), structure (Reyners, 1998), and frictional properties (Moore and Rymer, 2007), have been inferred from seismic velocities imaged through tomographic studies, and proposed as potential explanations for the occurrence and spatial distribution of SSEs in New Zealand (Wallace et al., 2009; Bassett et al. 2014; Reyners et al., 2017; Heise et al., 2017; Warren-Smith et al., 2019; Wallace, 2020). Most SSEs occur on subduction interfaces at depths corresponding to the lower limit of the seismogenic zone (Hirose et al. 1999; Dragert et al., 2001; Ohta et al., 2006; Wallace and Beavan, 2010; Radiguet et al., 2012, Dixon et al., 2014), and so explanations for their occurrence are intrinsically tied to the variability in slip rate deficit observed along the Hikurangi margin.

High-resolution images of crustal structure beneath the North Island will undoubtedly provide additional constraints and evidence for determining differences in physical properties between the northern and southern Hikurangi margins. Additionally they will provide more detailed images of the surrounding tectonic features, such as the Taupō Volcanic Zone, onshore and offshore sedimentary basins, and the interplay of geologic features in the North Island.

\subsection{Seismic tomography}

Tomography is a technique used to generate images of a solid object through the use of penetrating waves (Herman, 2009). In seismic tomography, seismic waves from passive (e.g. earthquakes) or active (e.g. explosions) sources, are used to image structure at many spatial scales (Liu and Gu, 2012). Tomography works by comparing differences between observations and predictions (synthetics) from a given model. By adjusting the model and its resultant outputs to agree with observations, tomography searches for a best-fitting model which most accurately predicts observations. This section provides a brief overview of earthquake-based seismic tomography. Adjoint tomography will be discussed further in Section 2.1.5. A detailed history of seismic tomography can be found in Liu and Gu (2012), and references therein.

Tomography involves finding solutions to the forward and inverse problems. In 
the forward problem, a model of a system is used to make predictions of measurements. In the inverse problem, measurements are used to characterize a system and create a model that can predict data. Here we follow the methods of solving the inverse problem laid out by Tarantola (2005). In the forward problem, characterizing a system allows one to map from a model $\mathbf{m}$, to predicted measurements $\mathbf{d}$, through the generalized system: $\mathbf{G}(\mathbf{m})=\mathbf{d}$. In seismic tomography, $\mathbf{m}$ often characterizes the Earth in terms of physical parameters such as seismic velocities, while $\mathbf{d}$ is typically comprised of seismic data like arrival times or seismic waveforms (Tarantola, 2005). The forward operator $\mathrm{G}$ is a physical principle that links the two, such as ray theory (Section 1.3.1) or the seismic wave equation (Section 1.3.3. In the related inverse problem, we seek a corresponding map to derive a model $\mathbf{m}$, using data $\mathbf{d}$. To do this we need to solve for the inverse of $\mathbf{G}$, or the generalized inverse operator. Note that depending on the complexity of $\mathbf{G}$, finding its inverse may be as trivial as taking $G^{-1}$. If $G$ is not invertible, then finding the generalized inverse $\mathrm{G}^{-g}$ can be considerably more difficult (Tarantola, 2005).

In general, the solution to the forward problem is unique, because it is a theoretical prediction based on physical laws. The solution to the inverse problem is non-unique, because there may be infinitely many models $\mathbf{m}$ which can reproduce the observed data $\mathbf{d}_{\text {obs }}$ (Tarantola, 2005). Additionally, errors in the forward problem are intrinsically mapped to the inverse problem through the generalized inverse $\mathbf{G}^{-g}$, therefore careful construction of $\mathbf{G}$, and the use of apriori information (e.g. choice of starting model), are important for constraining the inverse problem. Efforts to suppress non-uniqueness often require techniques which impose smoothness in an inversion procedure (Section 2.1.7, Modrak and Tromp, 2016). Much of tomography is centered on posing the forward and inverse problems correctly, such that the solutions that arise are as close to reality as possible (Tarantola. 2005).

We note that other forms of geophysical imaging besides tomography are commonly employed. Refraction seismology for example, estimates thicknesses and velocities of layers in the Earth using traveltime curves and ray theory, however this method is inherently two-dimensional, and typically requires the Earth to be a layered model, breaking down with increasingly complex structure (Stein and Wysession, 2009). Similarly, reflection seismology can produce detailed sections of Earth structure by taking advantage of reflected seismic waves, but shares many 
of the inherent difficulties of refraction seismology (Stein and Wysession, 2009). Interfometric techniques such as ambient noise tomography, which make use of large scale passive sources like ocean-generated waves, are elegant in that they only require information from seismographs (Snieder et al., 2010). However, resolution with these methods is limited due to the dominance of low frequencies from natural sources. Historically, seismic tomography has been the primary method for providing detailed 3D images of Earth structure (Liu and $\mathrm{Gu}, 2012$ ).

\subsubsection{Ray tomography}

Solving the elastic wave equation in complex media is computationally exhaustive, and early seismic tomography efforts starting in the 1980s were impeded by limitations in computational power and storage (Liu and Gu, 2012). To simplify this, forward modeling of the wave equation was first accomplished using ray theory (Aki and Lee, 1976), a high-frequency approximation based on Snell's law (Descartes and Laurence, 1631). Ray theory tracks a point on the propagating wavefront, approximating seismic waves as geometrical lines connecting source and receiver. Traveltimes are therefore related to the summation of seismic wavespeeds along the geometrical ray path (Liu and $\mathrm{Gu}, 2012$ ). The forward problem can be solved by "shooting" or "bending" rays through a model to predict traveltimes (e.g. Julian et al. 1977), while the inverse problem perturbs the velocities along the geometrical ray path for many source receiver pairs simultaneously, to reduce differences between observed and predicted traveltimes (e.g Thurber. 1993). With ray theory, the length-scale of heterogeneities within the medium must be larger than the seismic wavelength such that the Earth is approximated as smoothly varying media. The assumptions in ray theory are consequently no longer valid for complex, heterogeneous media (Stein and Wysession, 2009). Nevertheless, ray theory greatly simplifies analysis, and has typically provided a good approximation for seismic waves (Stein and Wysession, 2009).

\subsubsection{Finite-frequency tomography}

In ray theory, the sensitivity of seismic waves depends only upon the wave speeds along the geometrical ray path (e.g. Marquering et al., 1999). Its "sensitivity kernel", which relates perturbations in seismic velocities to changes in the predicted 
traveltimes, is confined to the geometric ray. However, ray theory is an infinitefrequency approximation, and finite-frequency wave propagation effects like scattering and diffraction should extend sensitivity to 3D structure off the geometrical ray path. In the famous "banana-doughnut paradox", Marquering et al. (1999) showed that the sensitivity kernel of a finite-frequency teleseismic $S$ wave was actually zero along the geometric ray path, and resembled the shape of a hollow banana (Figure 1.4). When these "finite-frequency sensitivity kernels" were incorporated into tomographic inversions (e.g. Marquering et al., 1999; Dahlen et al., 2000) the resultant images showed departure from earlier tomographic models, for example through inferred images of hot rising mantle plumes (e.g. Montelli et al., 2004a). It should be noted that some have argued over the quality of improvement from finite-frequency effects, and the need to account for them in ray-based tomography (Dahlen and Nolet, 2005: De Hoop and van Der Hilst, 2005; Montelli et al., 2006: Van Der Hilst and De Hoop, 2005).

The reason for requiring finite-frequency effects lies in the physics of wave propagation. In ray theory, waves retain the time shifts caused by velocity anomalies along their path. With finite frequencies however, diffracted waves can fill in or heal irregularities along the wavefront caused by strong anomalies, a phenomenon known as "wavefront healing" (Nolet and Dahlen, 2000; Hung et al., 2001; Malcolm and Trampert, 2011). This means that finite-frequency effects interfere with traveltime measurements, leading to inconsistencies in the inverse problem stemming from ray theory approximations (Montelli et al., 2004b). Ultimately, if the scale lengths of velocity heterogeneities are comparable to the wavelengths of the seismic waves being used for tomography, finite-frequency effects become important and should be considered (Montelli et al., 2004b).

\subsubsection{Full-waveform tomography}

Classical tomography efforts usually invert for traveltime data of a few prominent seismic phases that are easily picked from the waveform. However, the full broadband seismic waveform is rich with information. It seems intuitive that we would find improved accuracy and resolution if we include measurements from the "full" seismic waveform within an appropriate frequency band. We use the term fullwaveform tomography (FWT) to refer to any imaging efforts which measure all or part of the time-dependent seismic waveform, rather than point measurements 

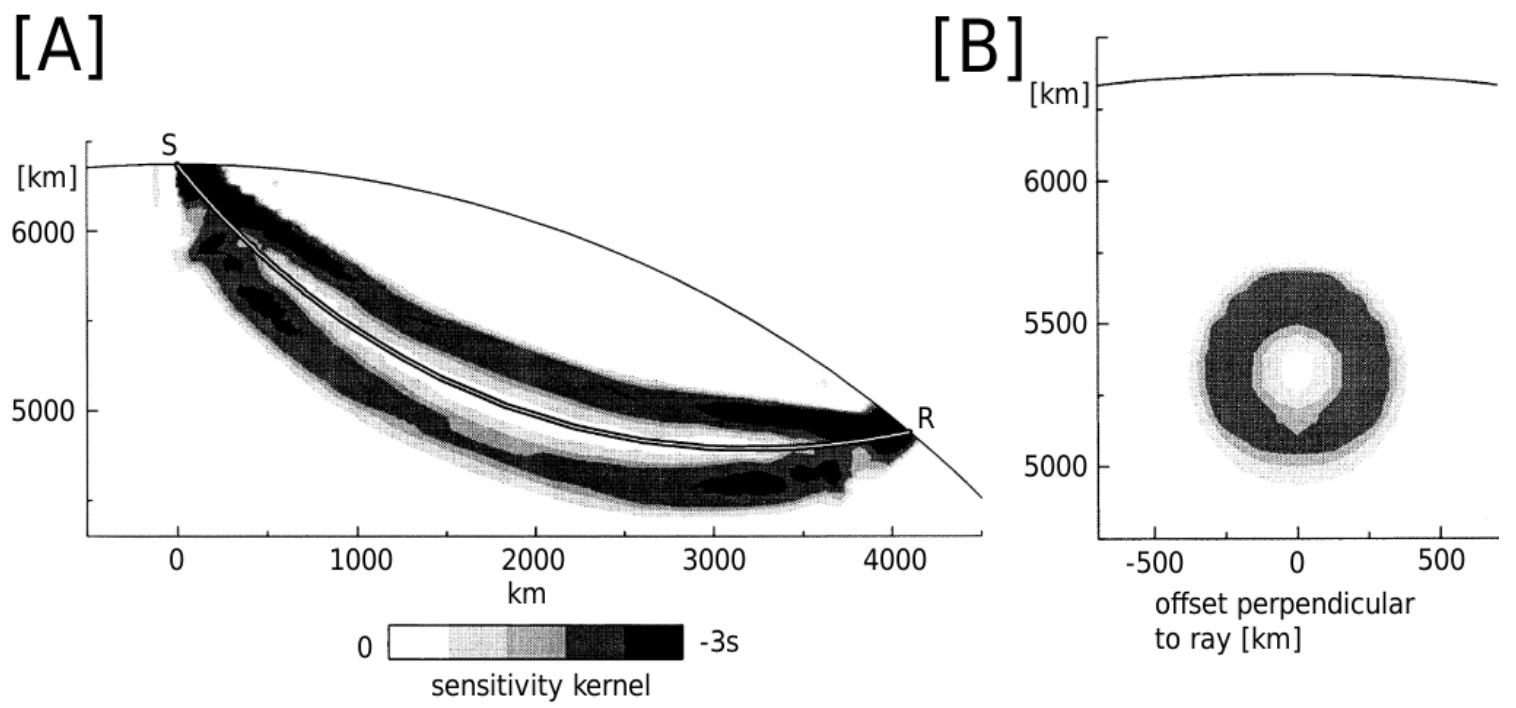

Figure 1.4: The banana-doughnut paradox, after Marquering et al. (1999). This 3D sensitivity kernel shows off-axis sensitivity, and zero sensitivity along the geometrical raypath, for a 17-100 s period $S$ wave at an epicentral distance of $40^{\circ}$. A) Ray-plane cross-section through the source (S), receiver (R), and center of the Earth, i.e. the "banana". B) Perpendicular cross-section through the turning point at $20^{\circ}$, i.e. the "doughnut".

like traveltime or amplitude differences. Adjoint tomography consequently constitutes a subset of FWT. Full-waveform methods approximate solutions to the wave equation, as opposed to the previously discussed ray approximations (Liu and $\mathrm{Gu}, 2012$ ).

With the advent of high-performance computers and improvement in numerical modeling techniques, it became feasible to solve the elastic wave equation using numerical methods for realistic problems. Full 3D simulations of seismic wave propagation in complex models have been realized since the early 2000s (Komatitsch and Tromp, 2002a). The resulting benefits of full-waveform tomography are numerous, and were listed explicitly by Tape (2009). Firstly, solving the wave equation means the complexities of wave propagation, such as finite-frequency effects, are implicit. Second, the entire waveform can be used for misfit measurements, as opposed to solely travel times. This allows for an increase in the number of measurements, and for the use of exotic seismic phases such as diffracted surface waves (Tape et al. 2010). Third, any part of the waveform can be used to make measurement, e.g. phases, amplitudes, or combinations thereof. This is in contrast to ray tomography, which can readily predict arrival times but not amplitude information (Stein and Wysession, 2009). 
The drawbacks of full-waveform tomography are primarily related to accessibility: at present, complex numerical solvers and supercomputers are required to simulate 3D seismic wave propagation in complex, heterogeneous media, and derive meaningful velocity models. Fortunately, various numerical solvers exist to tackle the simulation of seismic wave propagation (Komatitsch and Vilotte, 1998; Komatitsch and Tromp, 2002a b;: Gokhberg and Fichtner, 2016; Afanasiev et al., 2019). Similarly, data storage of models, kernels, waveforms etc., necessary to solve the forward and inverse problems, push the limits of modern clusters, while complexity scales as tomography implements larger domains, higher resolutions, and increased data. Nevertheless, the trade-offs of full-waveform methods are attractive given the physical accuracy they provide, and much of the current research in the field is focused on optimizing the forward and inverse problems through novel approaches such as wavefield adapted meshes (Thrastarson et al., 2020), or dynamic batching (van Driel et al., 2020; van Herwaarden et al., 2020).

\subsection{Tomography in New Zealand}

The largest tomographic imaging effort in New Zealand has been performed by Donna Eberhart-Phillips and colleagues. Most recently, their work has led to the NZ-Wide2.2 velocity model (Eberhart-Phillips et al., 2020b), developed using raybased traveltime tomography, and improved in areas with joint inversions of Rayleighwave group velocity maps (Eberhart-Phillips and Fry, 2017), and joint inversions with teleseismic surface waves (Eberhart-Phillips and Fry, 2018). The data used in the inversion include earthquake $P$ and $S$ arrival times as well as a small number of offshore active source arrivals. This $3 \mathrm{D}$ velocity model defines $\mathrm{V}_{p}, \mathrm{~V}_{p} / \mathrm{V}_{s}$, density and attenuation $\left(Q_{p}, Q_{s}\right.$; Eberhart-Phillips et al., 2015, 2017, 2020a) for the entire New Zealand region. In addition to these NZ-Wide tomography models, ambient noise tomography has been employed to image structure in New Zealand, both at the regional scale (Lin et al., 2007), and specifically targeting the Taupō Volcanic Zone (Behr et al., 2011). This thesis is the first application of adjoint tomography in New Zealand. 


\subsection{Thesis structure}

This thesis is divided into six chapters, including this introduction. Chapter 2 pertains to the numerical methods and seismic data used in this thesis, and contains a section on the Broadband East Coast Network (BEACON), a temporary seismic deployment specifically designed to improve coverage for our seismic imaging efforts.

Chapters 3, 4, and 5 were written with the intent of standalone publication in peer-reviewed journals. To reflect the contribution of multiple coauthors in these manuscripts, these chapters are written in the first-person plural, "we". For consistency throughout the thesis, the rest of the chapters also use the first-person plural ("we"), however the work in the thesis was performed by myself, Bryant, unless explicitly stated otherwise.

Chapter 3 was published in Geophysical Journal International with the following title: "An automated workflow for adjoint tomography — waveform misfits and synthetic inversions for the North Island, New Zealand". This chapter details the development and verification of a set of automated programmatic tools for adjoint tomography. These tools are used to assess a ray-based 3D velocity model of New Zealand for suitability in adjoint tomography. In the corresponding publication, all work and writing was performed by myself, Bryant. The named co-authors contributed technical and scientific direction throughout the research, and critical feedback of the manuscript.

Chapters 4 and 5 were written with intent for publication. As of August 2021, work based on the results presented in these chapters is under peer review as a set of companion manuscripts:

1. Bryant Chow, Yoshihiro Kaneko, Carl Tape, Ryan Modrak, Nick Mortimer, Stephen Bannister, John Townend, Strong upper-plate heterogeneity at the Hikurangi subduction margin (North Island, New Zealand) imaged by adjoint tomography, Journal of Geophysical Research: Solid Earth

2. Bryant Chow, Yoshihiro Kaneko, John Townend, Evidence for deeply-subducted lower-plate seamounts at the Hikurangi subduction margin: implications for seismic and aseismic behavior, Journal of Geophysical Research: Solid Earth 
All analysis and writing in these manuscripts was performed by myself, Bryant. The named co-authors on each manuscript contributed scientific direction throughout the research, and critical feedback on the manuscripts themselves.

Chapter 4 details a full-scale, high-resolution tomographic inversion of velocity structure for the North Island of New Zealand. The data and methodologies are largely similar to previous chapters, but there is some repetition so we can be explicit about the exact inversion inputs.

Chapter 5 provides an interpretive look at the updated velocity model. We discuss the most striking velocity changes with respect to the initial model, and compare revised velocity structure to known New Zealand tectonics and geology. We make tectonic interpretations of these features and discuss their implications.

Chapter 6 closes the thesis by revisiting the objectives laid out in this introduction, and proposing potential avenues for future work. 


\section{Chapter 2}

\section{Methods and data}

In the following chapter we discuss the methodologies and data used in this thesis. We start with methods, as the approach of adjoint tomography will motivate our choice of data. Section 2.1 provides a detailed look at the components of adjoint tomography, while Section 2.2 outlines our study area, and the selection of earthquakes and seismic stations within it. Section 2.3 provides a detailed look at the temporary seismic network, BEACON.

\subsection{Methods}

The underlying goal of seismic tomography is to fit observations of seismic data to predictions of the data. These predictions are generated through approximations of physical laws (e.g. ray theory, wave equation), using a model that estimates the structure which gave rise to the data. Through targeted changes, or perturbations, of the starting model, one can better fit predictions, and consequently generate a new model that is a more accurate image of real structure.

Adjoint tomography can be divided into multiple sub-problems, each requiring its own specialized tools and theory. Tomography typically involves performing many tedious mathematical computations and consequently the name of the game here is computational efficiency; methods are chosen for their ability to solve a given problem efficiently. 


\subsubsection{The forward problem}

One of the limiting factors in tomographic inversions is the accuracy of synthetics; accurate forward modeling is essential for accurate imaging. Solving the wave equation as a basis for forward modelling allows for the implicit honoring of complex wave propagation effects (Section 1.3.3). In adjoint tomography, we often seek solutions to the three-dimensional wave equation, which is a second-order linear partial differential equation of the form:

$$
\nabla^{2} \cdot \mathbf{u}(\mathbf{x}, t)=c^{2} \cdot \frac{d^{2} \mathbf{u}(\mathbf{x}, t)}{d t^{2}}+f(\mathbf{x}, t)
$$

where $\mathbf{u}$ is a measure of motion in the form of displacement or its derivatives at some point $\mathrm{x}$ and time $t$. The quantity $c$ represents velocity, describing the medium through which a wave travels, and $f$ is an optional forcing term. In theory, it is possible to generate analytical solutions to the three-dimensional wave equation (e.g. Kennett, 2009), but these methods require simplifying and often limiting assumptions. If $c$ is allowed to vary arbitrarily with space, as it does in true Earth structure, then numerical methods are required to attain solutions to the wave equation (Igel, 2017).

In this work, we use the term "simulation" to describe finding numerical approximations of $\mathbf{u}(\mathbf{x}, t)$, or time and space dependent solutions to Equation 2.1. Our synthetic earthquakes are represented by $f$, and result in a simulated wavefield $\mathbf{u}$, which propagates through a model defined by $c$. In a simulation, the seismic wavefield is defined at all points in the domain $x$, and at all times $t$. Retaining the full wavefield at each point in $\mathbf{x}$ and $t$ is possible, but requires massive storage capacity. For efficiency, we follow pragmatism and store the wavefield $\mathbf{u}(\mathbf{x}, t)$ only at a given set of synthetic station locations, which we can then compare with corresponding observations.

Equation 2.1 dictates that any differences between our observed waveforms and synthetic waveforms can only be introduced through differences in the forcing term $f$, and velocity model $c$. In this work, we assume the seismic moment tensor defined by $f$ to be correct, and that all differences must be attributed to differences between the velocity model $c$, and true Earth structure. 


\subsubsection{The spectral element method}

The spectral element method (SEM) is a numerical approximation schema first derived for fluid dynamics (Patera, 1984), and later formulated for elastic wave problems (Priolo et al., 1994; Seriani and Priolo, 1994; Faccioli et al., 1996). In this work we use the spectral element method to find solutions to the seismic wave equation (Equation 2.1). Although a full derivation is outside the scope of this thesis, we will highlight some of the inherent strengths in the method as motivation for why it was chosen for this work. The information presented here is summarized from Igel (2017). We re-iterate that in the forward problem, our goal is to find solutions to the seismic wave equation (Equation 2.1), which involves calculating time and space derivatives of a function $\mathbf{u}(\mathbf{x}, t)$.

The "spectral" part of spectral elements comes from the fact that this numerical method solves equations in the spectral, or Fourier, domain. The spectral element method approximates the wave equation using a set of basis functions (e.g. Lagrange polynomials) which correspond exactly to the function being approximated, at a specific set of collocation points (Figure 2.1). The power of exact derivatives, or spectral convergence, comes from the implementation of Fourier transforms (Gazdag, 1981), which allows for high accuracy of derivative calculations. By choosing a unique set of points to interpolate our function, such as GaussLobatto-Legendre (GLL) points from Komatitsch and Vilotte (1998) (Figure 2.1), we are able to massively simplify the amount of computation necessary to find solutions to the wave equation. In mathematical terminology, we are able to diagonalize a large matrix before taking its inverse, greatly reducing the amount of calculations required to approximate the wave equation. In other numerical methods common to seismology (e.g. finite element method), a non-diagonal mass matrix requires inverting a large, sparsely populated matrix, which is computationally prohibitive.

The "element" in spectral elements comes from the finite element method (Zienkiewicz and Taylor, 1989); element-based methods can form arbitrary element shapes by dividing the full domain into elements which behave identically and independently (Figure 2.2; Igel, 2017). These elements must only communicate at their contact points, allowing for complex geometries to be realized, including topography and internal discontinuities (Figure 2.3). As a bonus, the free-surface bound- 

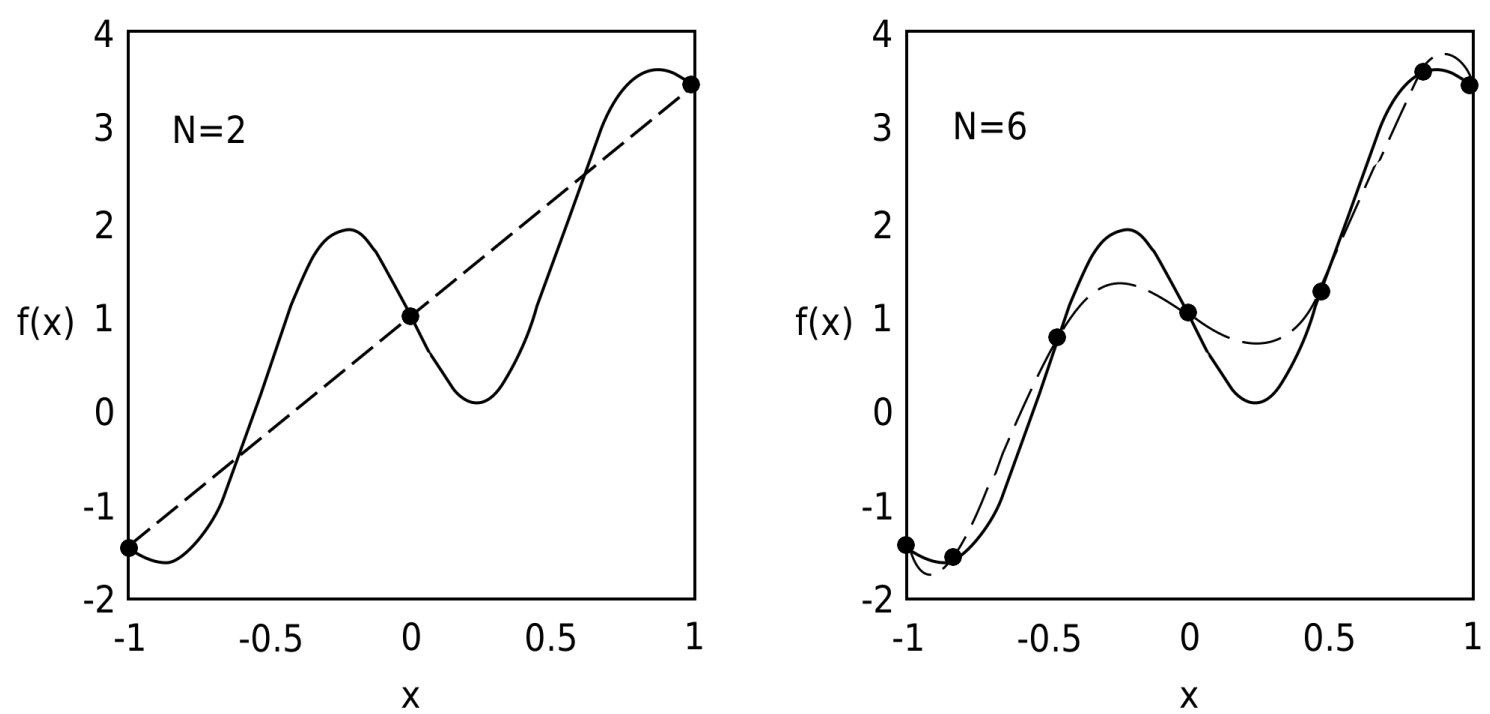

Figure 2.1: Polynomial interpolation and spectral convergence; figure modified from Igel (2017). Interpolation of a curve (black lines) using Lagrange polynomials (dashed lines) for $\mathrm{N}=2$ GLL points (left) and N=6 GLL points (right), corresponding to 3 and 7 grid points, respectively. Interpolation is exact at the GLL points (black circles). Spectral element simulations run using SPECFEM typically use N=5 GLL points.

ary condition is implemented in the derivation of this method, implicitly allowing for topography, something that is difficult to implement in methods which require rigid definitions of grid spacing such as the classical finite-difference method (Igel. 2017).

One drawback of this element-based approach is that the numerical grids, or meshes, are computationally complex, and require extra care to be generated properly. However the pros listed heavily outweigh this con, and have made the spectral element method the de facto numerical method for computational seismology. In this work we use the spectral element code SPECFEM3D Cartesian ${ }^{1}$ (SPECFEM) to simulate wave propagation (Komatitsch and Tromp, 2002a b).

\subsubsection{Meshing}

"Mesh" is the name given to the skeleton of ordered grid points upon which we discretize the continuous world (e.g. Figure 2.2). A numerically accurate mesh is required for accurate synthetics, and consequently a large effort in solving the forward problem is devising a mesh that accurately represents the domain. Due to the

\footnotetext{
${ }^{1}$ https://geodynamics.org/cig/software/specfem3d/
} 
Element 1

Element 2

Element 3

Element 4

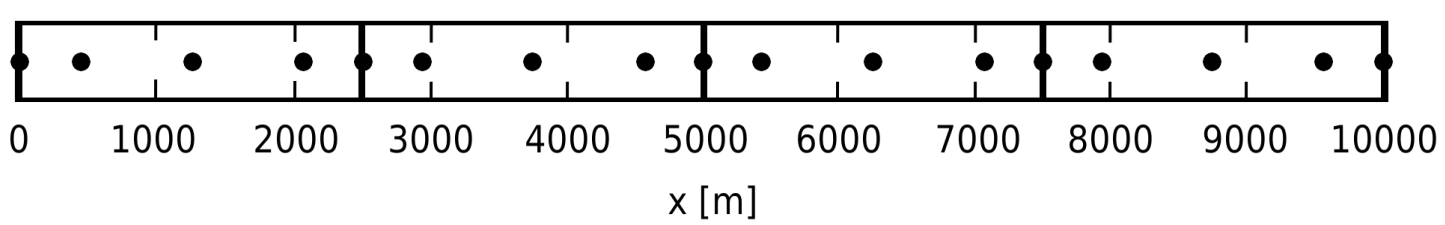

Figure 2.2: 1D spectral element domain decomposition, after Igel (2017). The entire physical domain $x \in[0,10000] \mathrm{m}$ is decomposed into $\mathrm{N}=4$ elements. Each element is further subdivided by a polynomial of order 4 ( 5 grid points representing GLL points). Elements only need to communicate at their edges (collocation points), drastically reducing computational overhead.

Courant-Friedrichs-Lewy condition (Courant et al., 1967), sampling high resolutions in space requires similarly high resolution in time to achieve numerical stability. Similarly, the Nyquist theorem (Shannon, 1949), requires at least two samples per dominant wavelength to accurately recreate the wavefield. These two conditions mandate that for a numerical mesh to accurately simulate high-frequency waves, it requires both small grid spacing, and frequent time steps, factors which increase computational cost.

Some steps can be taken to optimize meshes for the problem at hand. From $f=c / \lambda$, we can see that smaller wavespeeds $c$, relate to smaller wavelengths $\lambda$ for a fixed frequency $f$. Consequently, the controlling factor for resolution, grid spacing, and time step is the slowest wavespeed, normally found in the shallowest regions of a model (e.g. sedimentary basins). In the Earth, average wavespeeds generally increase with depth, allowing grid spacing to increase while retaining resolution. For this reason our numerical meshes have the smallest element sizes at the top, and feature interfaces at depth where elements double in horizontal size, and triple in vertical size (Figure 2.3). This reduces the number of elements in the mesh, while maintaining numerical and physical stability.

To generate meshes we use Meshfem3D, the internal meshing software of SPECFEM. Meshfem3D allows users to define mesh dimensions, element sizes, doubling and tripling layers, material properties, and interface topography. Interface topography can be used to define both surface values (topography, bathymetry) as well as internal surfaces (e.g. Moho geometry). Figure 2.3 shows a chunk of a Meshfem3D hexahedral mesh which features topography and internal coarsening layers. In 

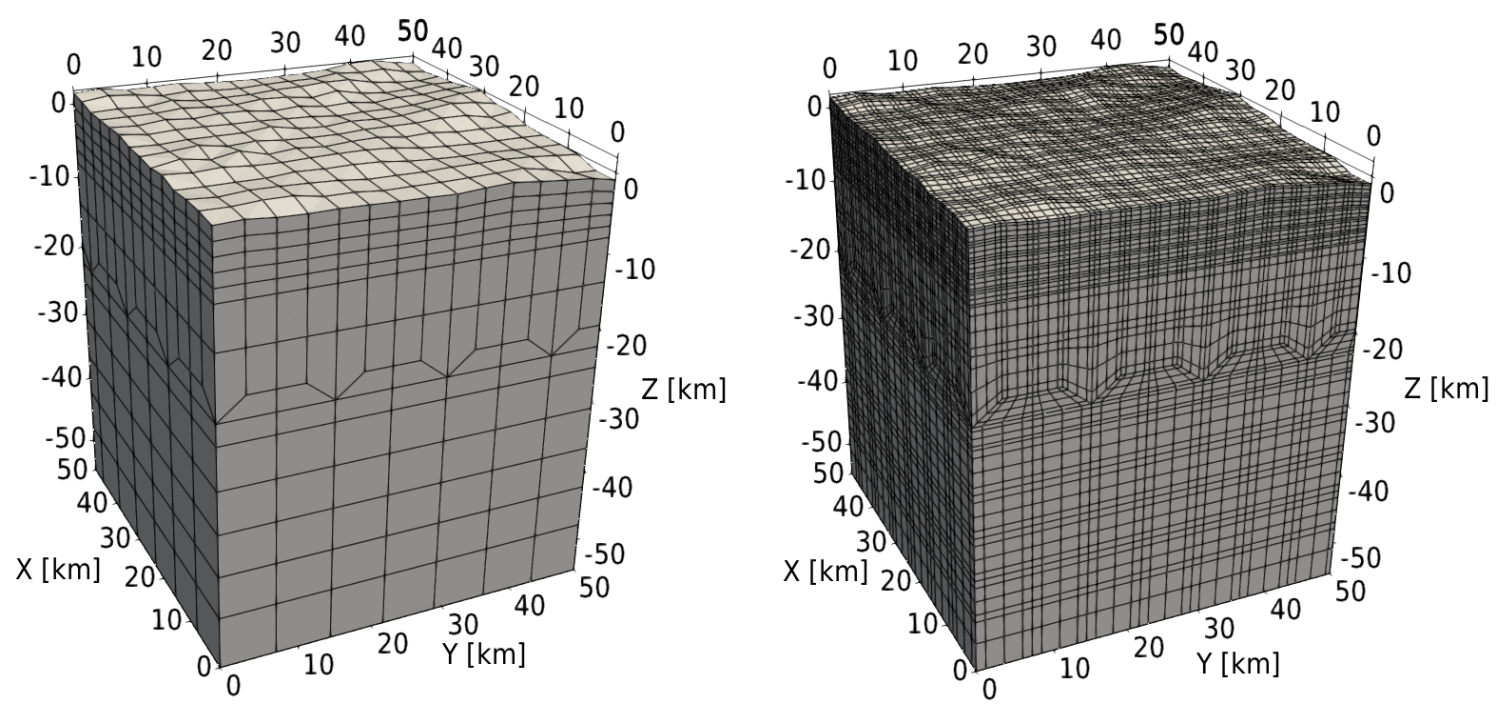

Figure 2.3: $\mathrm{A} \sim 50 \mathrm{~km}^{3}$ chunk of a spectral element mesh. The two sides show the same mesh, but the left side shows mesh element boundaries, and the right side shows Lagrange interpolation points (NGLL=5). Element sizes at the top are approximately $3.6 \times 3.8 \times 2 \mathrm{~km}$ in size, and show varying vertical element spacing due to the implementation of topography. At $8 \mathrm{~km}$ depth, a vertical tripling layer is visible where the vertical extent triples in dimension. Below $10 \mathrm{~km}$ depth, a horizontal doubling layer is visible where the lateral element dimension doubles. By $\sim 25 \mathrm{~km}$ depth, mesh coarsening is complete. The relationship between element boundaries and interpolation points is also shown in Figure 2.2 for a 1D example.

this work we define topography using SRTM-30P (Becker et al., 2009), while internal interfaces remain planar. The two parts of Figure 2.3 illustrate the relationship between element boundaries, and internal discretization by polynomial interpolation, which is shown for a 1D example in Figure 2.2 .

\subsubsection{The inverse problem}

The objective of our inverse problem is to minimize the misfit between data and synthetics, for example by reducing waveform or traveltime differences (Nolet. 1987; Tarantola, 2005). Given a starting model $\mathbf{m}$, we seek a perturbed model $\mathbf{m}+\delta \mathbf{m}$, which will minimize an objective function $\chi(\mathbf{m}+\delta \mathbf{m})$, quantifying datasynthetic misfit. We follow Tape et al. (2007) to formulate the inverse problem, and start by taking a quadratic Taylor expansion of the objective function $\chi(\mathbf{m}+\delta \mathbf{m})$ :

$$
\chi(\mathbf{m}+\delta \mathbf{m}) \approx \chi(\mathbf{m})+\mathbf{g}(\mathbf{m})^{T} \delta \mathbf{m}+\frac{1}{2} \delta \mathbf{m}^{T} \mathbf{H}(\mathbf{m}) \delta \mathbf{m}
$$


where the gradient vector, also known as the Fréchet derivative, is defined in terms of the first derivative of the objective function,

$$
\mathbf{g}(\mathbf{m})=\left.\frac{\partial \chi}{\partial \mathbf{m}}\right|_{\mathbf{m}},
$$

and the Hessian matrix is defined in terms of second derivatives of the objective function

$$
\mathbf{H}(\mathbf{m})=\left.\frac{\partial^{2} \chi}{\partial \mathbf{m} \partial \mathbf{m}}\right|_{\mathbf{m}} .
$$

The ' $\left.\right|_{\mathbf{m}}$ ' dependence is used to show that the preceding variable is evaluated at model $\mathbf{m}$ (Tape et al., 2007). The gradient of Equation 2.2 with respect to $\delta \mathbf{m}$ can be written

$$
\mathbf{g}(\mathbf{m}+\delta \mathbf{m}) \approx \mathbf{g}(\mathbf{m})+\mathbf{H}(\mathbf{m}) \delta \mathbf{m},
$$

which can be set to zero to obtain the local minimum of Equation 2.2

$$
\mathbf{H}(\mathbf{m}) \delta \mathbf{m}=-\mathbf{g}(\mathbf{m}) .
$$

We can find an updated model $\mathbf{m}+\delta \mathbf{m}$ with or without the Hessian $\mathbf{H}$; classical tomography is defined as inversions which can calculate the gradient and Hessian directly (Tape et al. 2007). When these terms are known, $\mathbf{m}+\delta \mathbf{m}$ can be obtained directly, and methods with access to the gradient and Hessian are known as Newton methods. When access to the gradient is available, but access to the Hessian is computationally prohibitive, one can use iterative techniques (Section 2.1.6) to minimize the objective function using only the gradient. These are known as gradient methods (Tape et al., 2007).

\subsubsection{The adjoint-state method}

Set by the formulation of our inverse problem (Section 2.1.4), our task is to find the gradient of an objective function (Equation 2.3). In seismic tomography, the model vector $\mathbf{m}$ is long, and therefore brute-force calculations of the derivatives in Equation 2.3 are generally computational infeasible (Tromp et al., 2005); we must turn to other methods to find the gradient.

The adjoint-state method (adjoint methods) is a numerical method for efficiently calculating the gradients of a function (Tarantola, 1984; Tromp et al., 2005; Fichtner et al. 2006a). The "state" in adjoint-state refers to the state equations, which 
are simply the equations that define the problem (i.e. the forward problem). The state equations relate the objective function to the model parameters (state variables); for example, the wave equation relates seismic wave speeds to predicted wavefields (Plessix, 2006). We are therefore interested in finding the derivatives of the state variables with respect to the model parameters (Fréchet derivatives; Equation 2.3). The "adjoint" refers to the idea that the method uses the adjoint of the state equations (conjugate transpose matrix) to determine the gradient. Adjoint methods consequently change the scale of the derivative calculation, from one proportional to the number of model parameters, to one proportional to the number of receivers, which is much more computationally tractable (Menke, 2018). We forgo a detailed derivation of the adjoint-state method, and instead refer the reader to Tromp et al. (2005); Plessix (2006); Fichtner et al. (2006a b); Tape et al. (2007); Menke (2018). Here we instead summarize, and point out some important aspects of, adjoint methods.

Adjoint methods are similar to the concept of "time-reversal mirrors" in the medical imaging field (Fink, 1992, 1997; Fink et al., 1989), where residuals recorded at receivers are time-reversed and re-transmitted, focusing at the original source location or at locations of model inaccuracies (Liu and Gu, 2012). In adjoint tomography, an "adjoint source" is a time-reversed signal re-transmitted from station locations; this is best illustrated visually, as in Figure 2.4. Similar to the definition of "simulation" (Section 2.1.1), an "adjoint simulation" describes finding solutions to an adjoint equation, which involves calculating time and space integrals for interactions between a forward wavefield $\mathbf{u}(\mathbf{x}, t)$, and an adjoint wavefield $\mathbf{u}^{\dagger}(\mathbf{x}, T-t)$ (where the adjoint wavefield is time reversed for a given simulation time $T$ ). During the adjoint simulation, the interaction between a forward wavefield, propagating forward in time, and the adjoint wavefield propagating backwards in time, produces the Fréchet derivatives, or the gradient of the objective function (Figure 2.4: Equation 2.3: Tromp et al., 2005).

When the adjoint source is proportional to synthetics only, then the summation of these Freéchet derivatives is the same as banana-doughnut finite-frequency kernels; to re-iterate Section 1.3.2, banana-doughnut kernels, or sensitivity kernels, relate predicted traveltimes to model perturbations (Tromp et al., 2005; Liu and Tromp, 2006). When the adjoint source is dependent on measurements between data and synthetics (i.e. the misfit), then the resulting sensitivity kernels illumi- 
nate parts of the model in which the data-synthetic misfit is sensitive to model perturbations (Tromp et al., 2005; Tape et al. 2007). These data-dependent kernels are termed "event kernels", and summations of event kernels are termed "misfit kernels". In adjoint tomography we produce event and misfit kernels, which represent the gradient of the data-synthetic misfit function (Equation 2.3).

In practice, the calculation of the gradient using adjoint methods requires two simulations per event: one forward simulation to calculate synthetics, and one adjoint simulation to generate the event kernel (Tromp et al., 2005). Individual event kernels can then be summed to represent the gradient of the misfit function. The cost of adjoint tomography therefore scales with the number of events $\mathrm{N}_{\mathrm{e}}$, requiring $2 \times \mathrm{N}_{\mathrm{e}}$ simulations to calculate the gradient. A single adjoint wavefield is composed of the summation of adjoint wavefields from all receivers for a given source, making this method independent of the number of receivers (Tromp et al. 2005). More importantly, adjoint methods are independent of the number of model parameters, which is not always the case when solving for Fréchet derivatives (Plessix, 2006). This allows adjoint tomography to honor much of the complexity of true Earth structure, at manageable computational costs.

\subsubsection{The L-BFGS algorithm}

The calculation of the Hessian for a 3-D reference model is generally not an option, and consequently one must turn to iterative, gradient-based algorithms (Tape et al. 2007). Nonlinear optimization algorithms involve finding extrema (minimum, maximum, or saddle) of objective functions, given a set of constraints (Modrak and Tromp, 2016); we consider the nonlinear regime because our objective function is nonlinear (Equation 2.2). A nonlinear optimization algorithm is designed to return a search direction and step length in which to perturb a given model, and defines a set of criteria to determine how many iterations should be required to find an extrema.

In this work we use the Limited-memory Broyden-Fletcher-Goldfarb-Shanno (LBFGS) algorithm for nonlinear optimization (Liu and Nocedal, 1989). During an inversion, the L-BFGS algorithm forms a quadratic model of the objective function, with each new gradient evaluation providing additional information. This "quasiNewton" method therefore approximates the Hessian using successive calcula- 

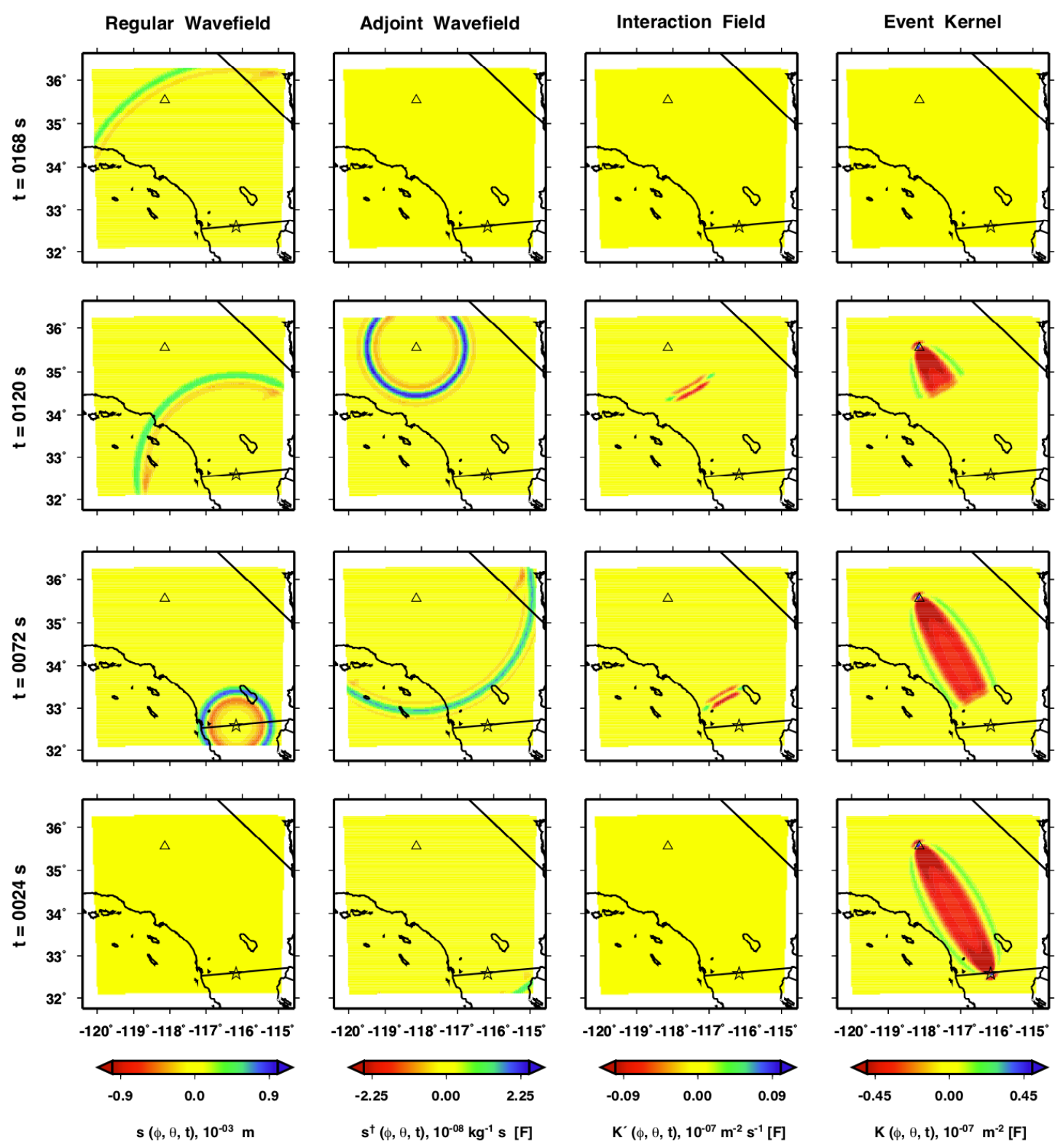

Figure 2.4: Construction of a cross-correlation event kernel K(x), from Tape et al. (2007). Each row represents a distinct time-step. The event kernel (fourth column) is constructed as the interaction (third column) of the forward, or regular, wavefield (first column), and the adjoint wavefield (second column). Source shown as a star, receiver shown as a triangle. Note the adjoint wavefield (second column) is propagated from receiver to source, and backwards in time. The "event kernel" illuminates the part of the model in which perturbations of model parameters affect misfit between data and synthetics. Summations of event kernels for many source-receiver pairs are called "misfit kernels" (Tape et al. 2007). 
tions of the gradient, as opposed to Newton methods which can calculate the Hessian directly. L-BFGS only retains the most recent gradient evaluations, hence the "limited-memory", and is shown to be more computationally efficient than other commonly used nonlinear optimization algorithms (Modrak and Tromp, 2016). The L-BFGS algorithm is stated explicitly in Modrak and Tromp (2016, Appendix A). Practically, we implement the L-BFGS optimization algorithm using SeisFlows (Section 3.3.1, Modrak et al., 2018).

\subsubsection{Regularization}

Regularization is the term given to measures taken to suppress non-uniqueness in an optimization problem (Modrak and Tromp, 2016). These measures are usually in the form of imposed smoothness, or direct constraints on the model or objective function. Regularization is used to guide, or steer optimization towards global minima. In this work, we regularize through convolution of the gradient with a 3D Gaussian function, which smooths the gradient over chosen length scales. This is accomplished practically with internal tools of SPECFEM.

Mathematically, the 1D Gaussian can be expressed as $G(x)=\left(2 \pi \sigma^{2}\right)^{-1 / 2} e^{-x^{2} /\left(2 \sigma^{2}\right)}$, where $\sigma$ is the half-width of the Gaussian; the full width of the Gaussian is given as $\Gamma=\sigma \sqrt{8}$. In 3D Cartesian coordinates, $\sigma$ can be expressed in the orthogonal directions $x, y$, and $z$. In this work we choose one horizontal scale-length $\sigma_{h}=\sigma_{x}=\sigma_{y}$ ( $h$ for horizontal), while the vertical scale-length $\sigma_{v}$ is typically smaller than $\sigma_{h}$ because of the varying sensitivity of surface waves to vertical and horizontal directions (e.g. Stein and Wysession, 2009). In practice, we choose smoothing lengths based on dominant wavelength, through qualitative tests with various trial smoothing lengths (Figure 2.5), and through comparisons with previous studies that make use of similar waveform bandpass and domain size (e.g. Tape et al. 2010).

\subsection{Data}

\subsubsection{Study area}

Our study area defines a region consisting of the central and southern North Island, and the northern South Island (Figure 2.6), with domain edges chosen pri- 

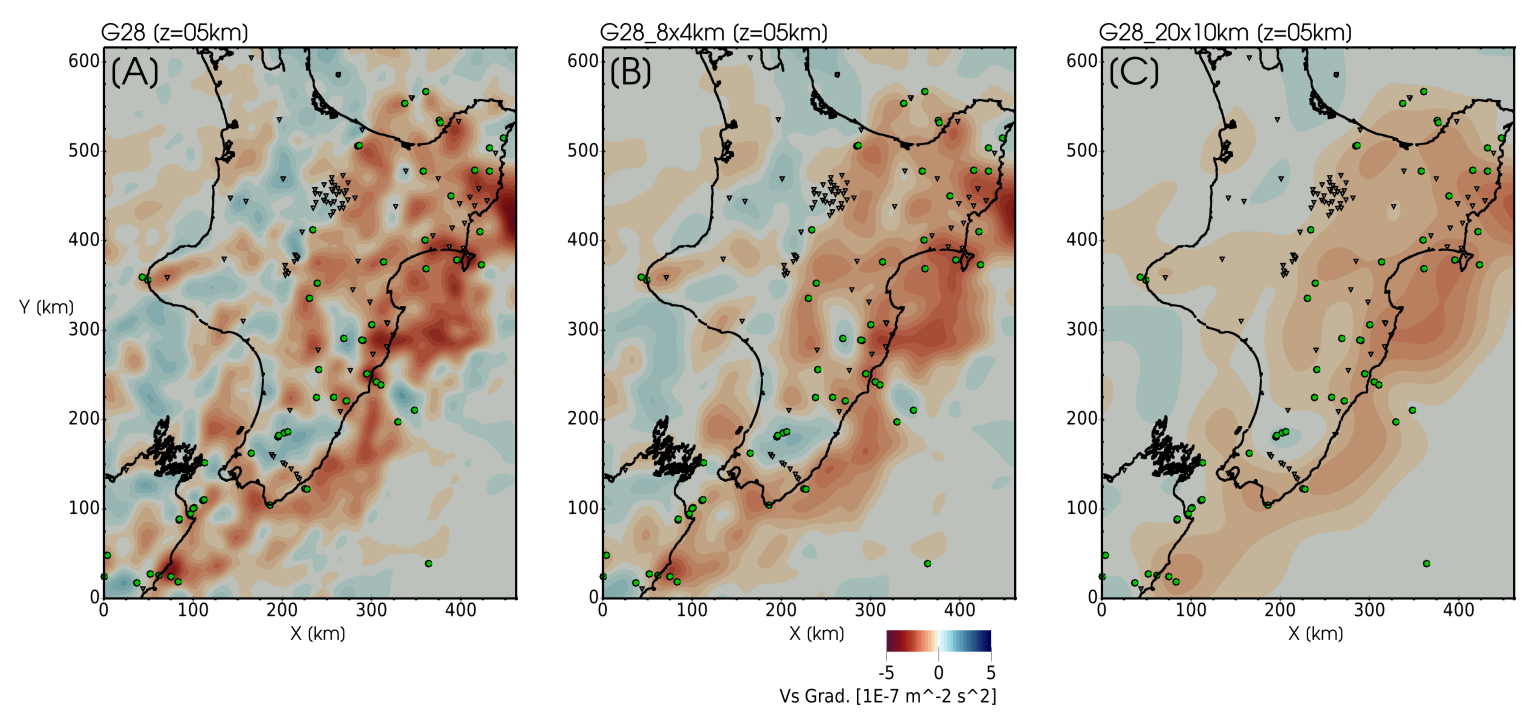

Figure 2.5: The effect of regularization by convolution with a 3D Gaussian, for an example gradient at $5 \mathrm{~km}$ depth. A) Raw gradient derived using 4-30 s waveforms and 1800 source-receiver pairs; sources and receivers shown as green circles and inverted triangles, respectively. B) Smoothing of the gradient in (A) with a 3D Gaussian with $\sigma_{h}=8 \mathrm{~km}$ and $\sigma_{v}=4 \mathrm{~km}$. C) Smoothing of the gradient in (A) with a 3D Gaussian with $\sigma_{h}=20 \mathrm{~km}$ and $\sigma_{v}=10 \mathrm{~km}$.

marily based on source and receiver locations, as well as concerns for computational expense. Limited station coverage, and a lack of large magnitude $\left(M_{w}>4\right)$, shallow (depth> $60 \mathrm{~km}$ ) events ruled out including regions north of Auckland $\left(37^{\circ} \mathrm{S}\right)$. The eastern boundary $\left(178.5^{\circ} \mathrm{E}\right)$ is limited to the sub-aerial extent of the North Island, chosen to minimize the amount of deep-ocean model space with limited to negligible data coverage. The southern $\left(42.5^{\circ} \mathrm{S}\right)$ and western $\left(173^{\circ} \mathrm{E}\right)$ boundaries were chosen to include a number of aftershocks and related seismicity from the $2016 \mathrm{M}_{\mathrm{w}} 7.8$ Kaikōura earthquake (Hamling et al., 2017; Holden et al. 2017). In this thesis, all locations are converted to, and shown in, the UTM 60S coordinate system. Our domain defines a rectangular grid covering an approximate size of $450 \mathrm{~km}$ in the X-direction (approximately east-west) by $600 \mathrm{~km}$ in the Y-direction (approximately north-south), by $400 \mathrm{~km}$ in the Z-direction (positive Z up).

\subsubsection{Earthquake sources}

For our tomographic inversion, we aim to select earthquakes with high signalto-noise ratio surface waves. Surface waves are preferred as they maximize the number of measurements with strong sensitivity to shallow crustal structure. We 
gather an initial catalog of events with magnitudes ranging $4.5 \leq \mathrm{M}_{\mathrm{w}}<6.0$, and depths shallower than $60 \mathrm{~km}$. High signal-to-noise ratio waveforms from events with magnitudes $\mathrm{M}_{\mathrm{w}}<4.5$ are most likely not recorded by all stations in the domain, limiting their usefulness in our regional scale inversion. Events with $\mathrm{M}_{\mathrm{w}} \geq 6.0$ are ruled out because our simulations represent earthquakes as point sources, and large magnitude events often require unique source time functions or finite-fault solutions for accurate synthetic waveforms. If we were to consider these larger events, any errors stemming from source characterization would arise as errors in our structural inversion. Moment tensors in New Zealand are routinely calculated by GeoNet ${ }^{2}$ (Ristau, 2008) and are historically available for regional earthquakes since 2003.

An initial catalog was gathered, consisting of roughly 400 suitable events ranging in time from 2004-2020. We manually performed quality control to compare observed and synthetic waveforms for GeoNet broadband stations, to ensure adequate signal-to-noise ratios, and sufficient initial waveform fit in the 10-30 s period range (Section 3.3.2). This step approximately halved the suitable events in the catalog to 250 (Figure 3.11). We select events from this catalog based on distributions in space and magnitude, to perform synthetic inversion (Section 3.4.1), the full-scale tomographic inversion, and a post-hoc waveform improvement validation (Chapter 4). Gathering and preparation of moment tensors is described in Appendix B

\subsubsection{Receivers}

Broadband seismic data were collected for 88 three-component broadband stations, consisting of 38 permanent network (GeoNet) and 55 temporary network stations in New Zealand (Figure 2.6). In total, 1800 unique source receiver paths are used, with temporary network data providing roughly $8 \%$ of the initial dataset. Here we detail the networks and data used. Waveform processing is described in detail in Section 3.3 .

The permanent seismic network of New Zealand is operated by GeoNet. To gather GeoNet data, raw waveforms and station metadata were collected for each earthquake in our catalog via the IRIS FDSN web services (Incorporated Research In-

\footnotetext{
${ }^{2}$ https:/ / www.geonet.org.nz/
} 
stitutions for Seismology International - Federation of Digital Seismographs Network). In practice, this was accomplished with Python using ObsPy (Beyreuther et al., 2010; Krischer et al., 2015b), and automated using Pyatoa (Section 3.3.2).

In addition to the permanent network of New Zealand, temporary seismometer deployments throughout the North Island are used to enhance coverage of the permanent network (Figure 2.6). In the southern North Island, the Seismic Array Hikurangi Experiment (SAHKE) transect consisted of a line of short-period seismic receivers deployed perpendicular to the trench to capture offshore shots and image plate interface characteristics (Figure 2.6: Henrys et al., 2013). Some of these sensors were collocated with broadband receivers, seven of which are included in our dataset. Raw waveform data and station metadata for the SAHKE experiment are publicly available through IRIS. Two additional deployments of seismometers, one focused on the Taupō Volcanic Zone, the other on the Gisborne region in the eastern North Island, were deployed by Stephen Bannister and colleagues (Figure 2.6). This increased our dataset by an additional 36 station locations.

In southern Hawke's Bay, the BEACON deployment targeted improving resolution over the shallow plate interface (Figure 2.6). In the next section we discuss deployment and operation of BEACON.

\subsection{The Broadband East Coast Network (BEACON)}

The Broadband East Coast Network, hereon referred to as BEACON, was a temporary deployment of broadband seismometers totalling 22 unique station locations. The network was deployed between July 19, 2017 and May 5, 2019, primarily in the southern Hawke's Bay region, with some stations in the eastern ManawatūWhanganui region. During operations, up to 18 stations were deployed at any one time. Network deployment and subsequent servicing was conducted by the following people: Yoshihiro Kaneko, Bryant Chow, Jonathan Hanson, Dan Whitaker, Rory Hart, Conrad Burton, Garth Archibald, Dan Bassett, Kris O’Brien, Tim McDougall, Hubert Zal, Danielle Lindsay, and Jesse Kearse. Here we provide an

\footnotetext{
${ }^{3}$ As of August 2021, BEACON waveform data and metadata are in the process of being uploaded to the Incorporated Research Institutions for Seismology Data Management Center (IRIS DMC) under the network code 2P. When available, data can be accessed through the following link: http://www.fdsn.org/networks/detail/2P_2017/ (last accessed Aug. 20, 2021).
} 


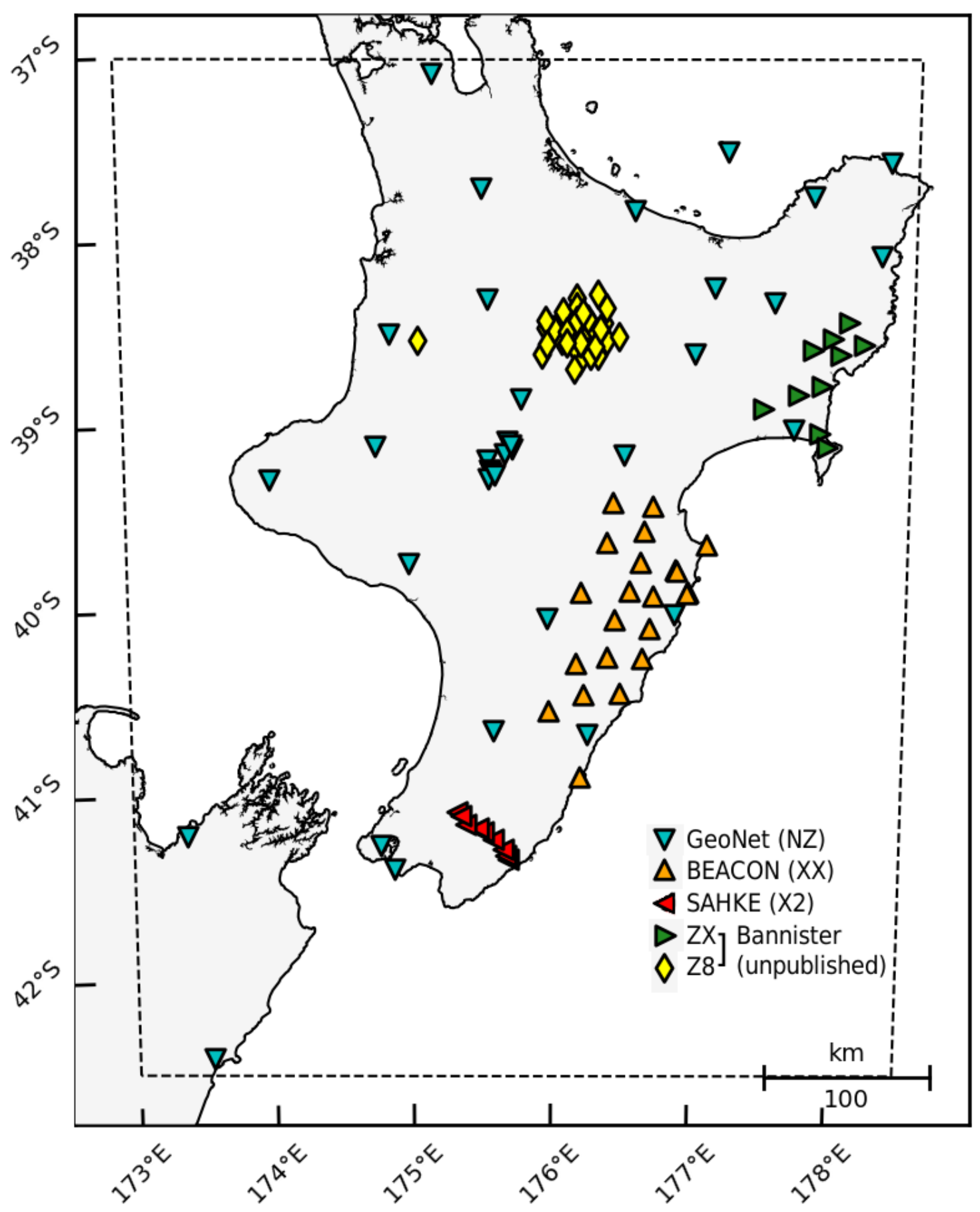

Figure 2.6: A receiver map showing the various networks used in this study. The dashed black line shows the simulation domain. Only GeoNet stations have continuous recording for all events in our catalog. All other networks are temporally limited. BEACON was deployed as a component of this thesis (Section 2.3). 
explanation of the network deployment and servicing efforts, network characteristics, and a data quality issue that was encountered.

\subsubsection{Deployment}

BEACON seismometers were a mix of Guralp 40T 30S and Guralp 40T 60S 3component broadband seismometers (Table 2.1). The use of broadband seismometer was motivated by the necessity of capturing long-period surface wave signals from regional earthquakes for adjoint tomography. Instruments were connected to Nanometrics Taurus dataloggers with 16 GB SD flashcards, and GPS antennae for precise timing. Dataloggers were set to $100 \mathrm{~Hz}(\mathrm{dt}=0.01 \mathrm{~s})$ sampling rate. Due to considerations for site deployment time, total network operation time ( $\sim 1.5$ years), and operations cost, no solar panels were used in the stations; power to stations was initially comprised of two 12 V HAZE solar gel batteries. At deployment time, it was estimated that two new batteries could last approximately two months before requiring recharge. Consequently, network servicing was to be performed once every two months, over the course of four days (4-5 stations per day). Partway through the deployment (late June, 2018), a third battery was added to each site to increase station uptime. Data cards had capacity for more than six months, and were estimated to far outlast battery capacity for a given servicing interval.

\section{Site selection}

The broad target region of BEACON was chosen to provide additional coverage over the geodetically identified locked-to-creeping transition zone (Figure 1.3). Site locations were selected based on current and previous GeoNet station locations (short-period seismometers or continuous GPS) (Table 2.1). This choice was based on the assumption that these areas had previously been scouted and vetted by GeoNet for accessibility and data quality. Additionally, in the case of co-located short-periods, GeoNet sites could be used to check timing and data quality. Scouting consisted of locating existing GeoNet installations, and searching the immediate vicinity for exposed bedrock; solid bedrock contact is important for seismic observation, as it equates to good coupling of the instrument to the ground. In some cases no bedrock could be found, and sites were installed directly in topsoil (Table 2.1). All stations were installed well away from cultural noise such as roads or townships, usually in farm paddocks. More than half of the sites required a 
light utility vehicle for access.

\section{Site installation \& servicing}

Sites were installed following standard temporary network installation best practices (Bormann, 2012). At each potential site location, multiple holes were dug until bedrock was found at a suitable depth $(\sim 1.5 \times$ height of seismometer). If no bedrock was found, sites were located near the closest rock outcrop. The bottom of the hole was filled with quick-drying concrete, and a stone paving slab was sunk into the still-wet concrete and levelled to provide a stable platform for the instrument (Figure 2.8A). The seismometer was then placed on the slab and levelled using the onboard bubble level (Figure 2.8B). The datalogger and batteries were hooked up and the station was powered on to ensure nominal performance, including: stomp test, GPS lock check, levelling, power, and storage capacity. Once checked, the seismometer was held firmly in place and covered with coarse sand, filling the hole; the sand was meant to provide additional coupling, and shielding from elements. The datalogger and GPS were stored inside a rugged case for protection, and placed atop the now-filled hole (Figure 2.8 C). A fish bin, or heavy plastic box, was placed on top of the entire assembly (datalogger, batteries, seismometer hole), and staked down at six points along its edge (Figure 2.8D).

Once every two months, network servicing was required to replace batteries and datacards. Each field session was performed over the course of four days, targeting four to five stations per day. At each site, the fish bin was removed and the data logger was checked. If running, a power down was initiated. The batteries were then disconnected and replaced, and a fresh 16GB datacard was installed. Two separate sets of data cards were used such that cards that were already written on would not be re-deployed during the same servicing period. We only had enough batteries to cover one day of field work, including one to two spares (10-12 batteries total), so we had to leapfrog our batteries; discharged batteries collected during the day were charged overnight, and then installed at sites the next day. With new batteries installed, we powered on the site and checked for nominal operation before replacing the fish bin. 


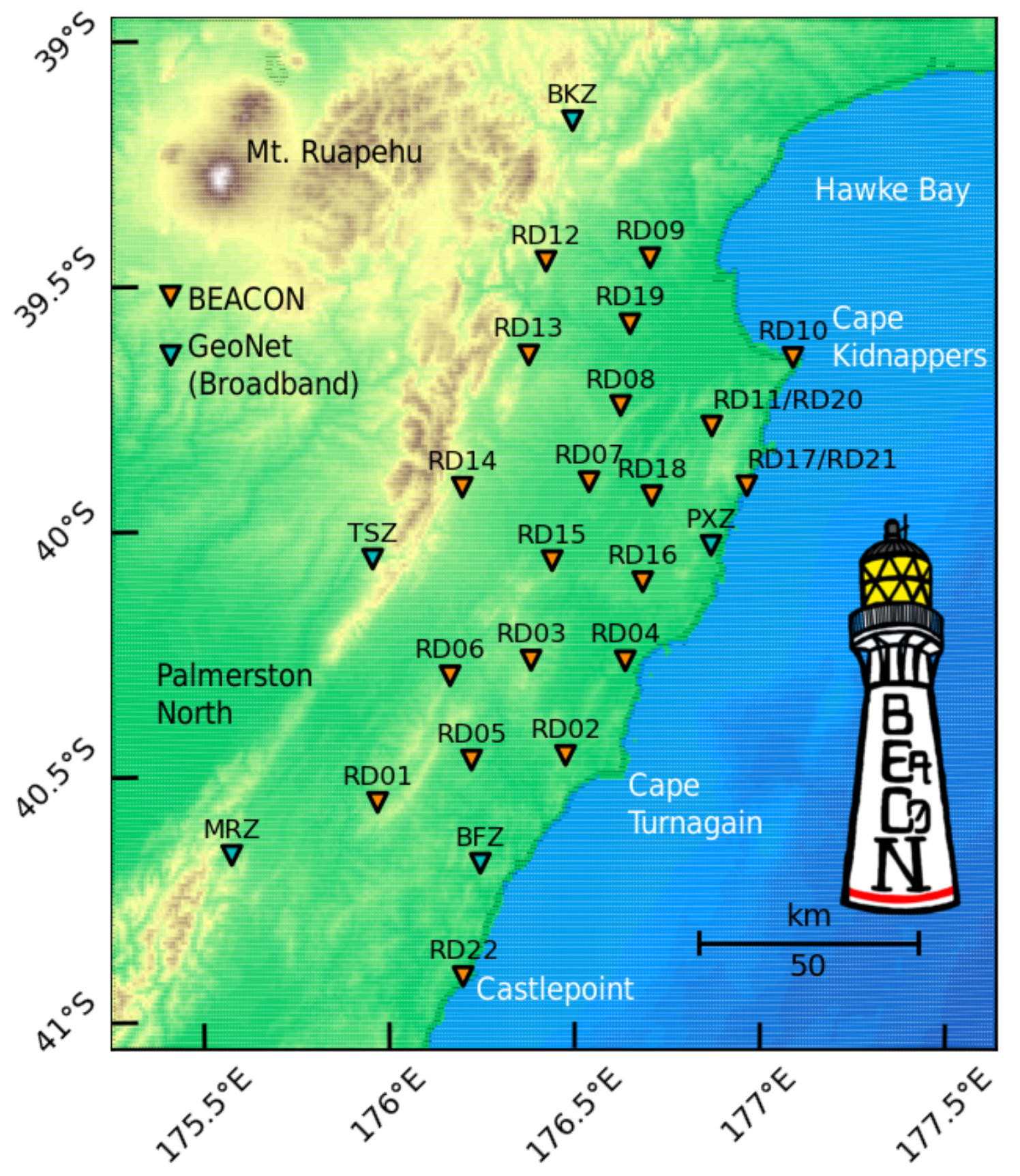

Figure 2.7: Station map centered on southern Hawke's Bay, showing BEACON station locations (orange inverted triangles), and surrounding GeoNet broadband stations (inverted blue triangles). BEACON logo inspired by Castlepoint lighthouse. 


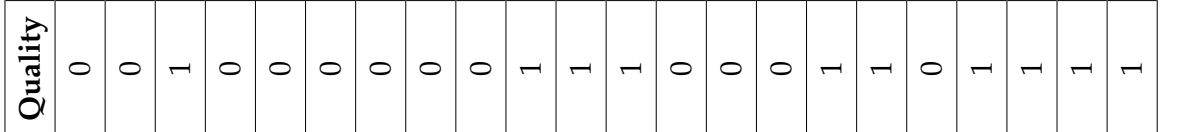

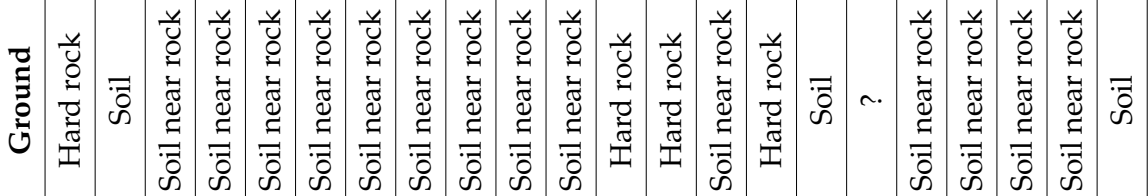

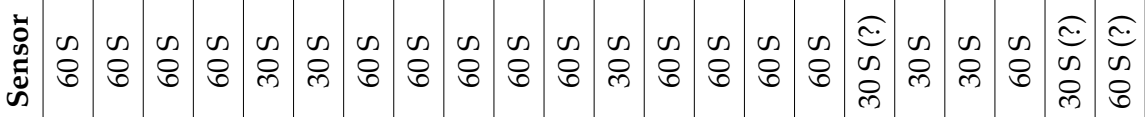

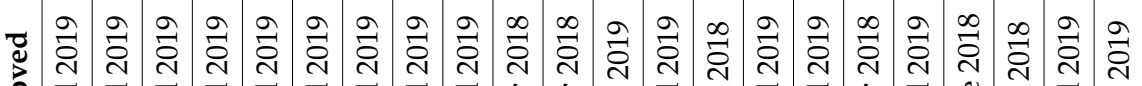

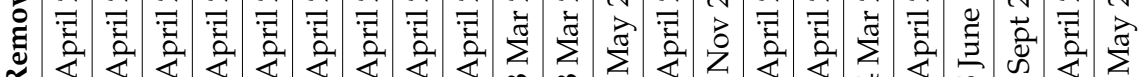

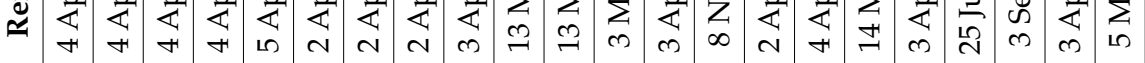

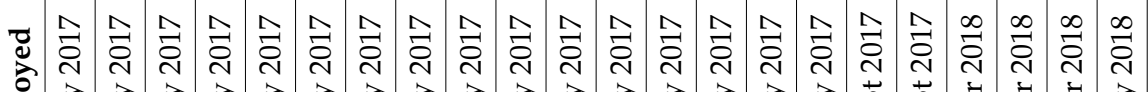

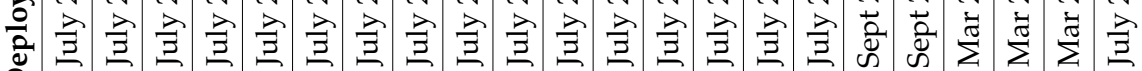

๑

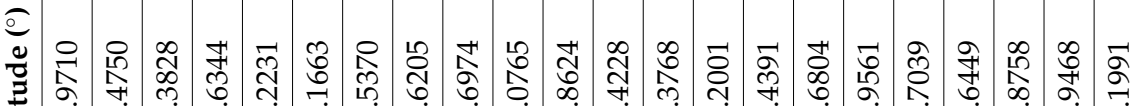

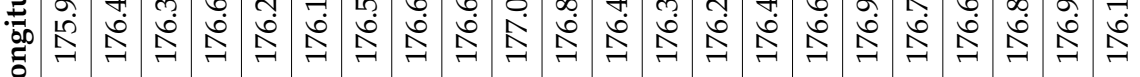

1

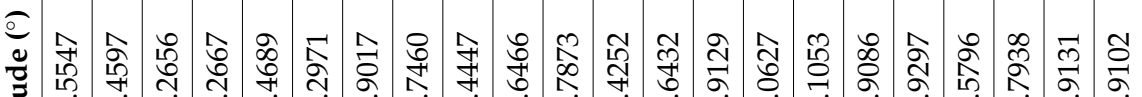

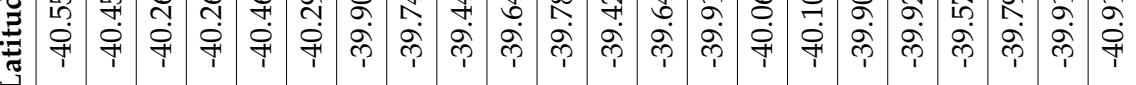

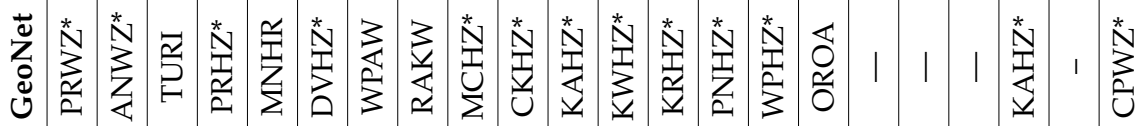

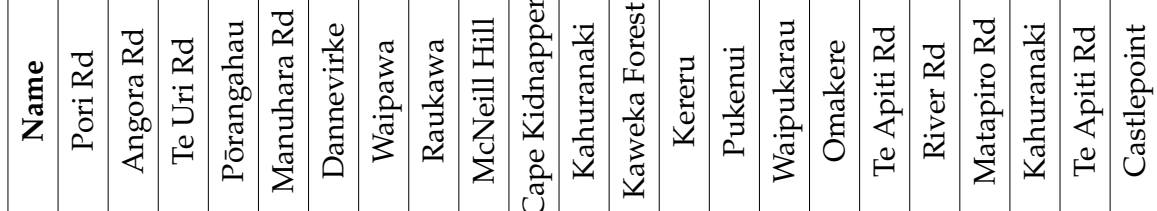

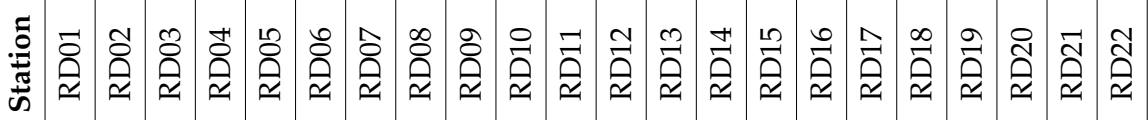

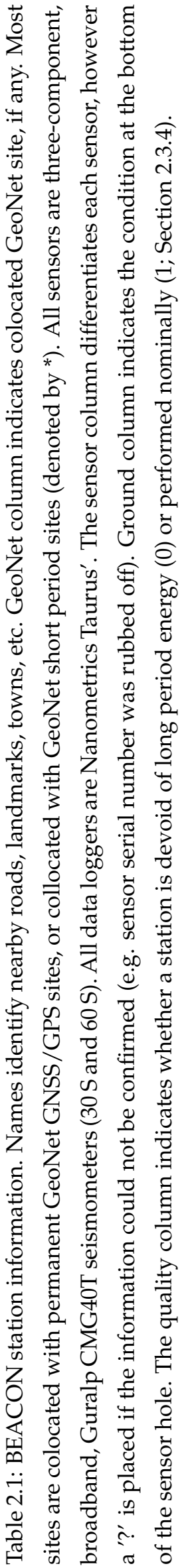




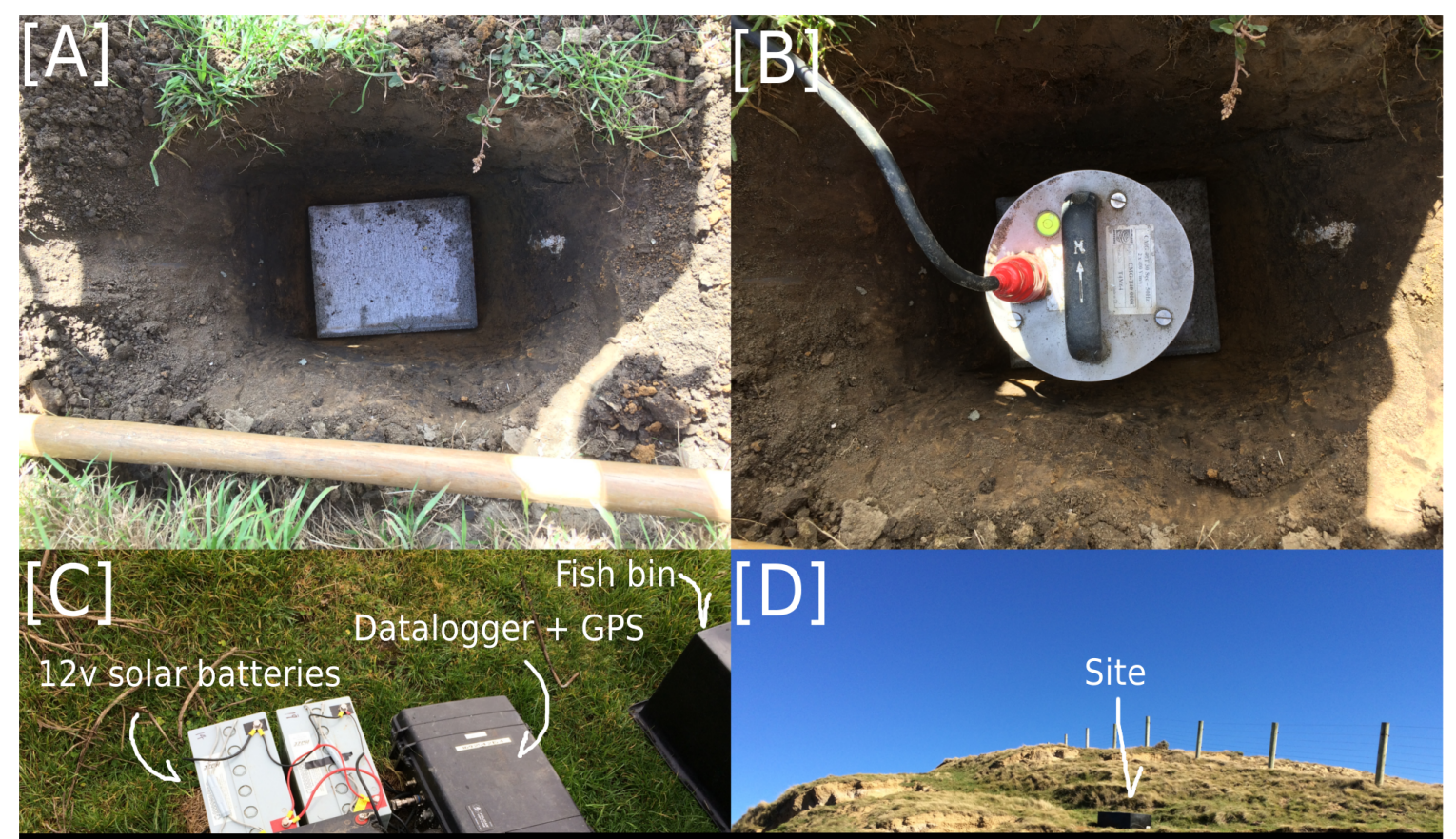

Figure 2.8: A BEACON example site installation. A) Top down view of hole, and paving slab concreted to bottom of hole for good coupling. B) Seismometer placed on top of paving stone and levelled using onboard bubble level. C) Complete site installation, showing seismometer buried in sand, covered with datalogger and GPS inside a rugged case, alongside $312 \mathrm{~V}$ solar gel batteries. The corner of the fish bin can be seen, which is secured on top of this configuration. D) Installed site (RD21, Castlepoint) from afar with only the fish bin visible. 


\subsubsection{Data availability}

About half a year into the deployment (beginning of 2018), we noticed that most sites had lost power by servicing (Figure 2.9). We diagnosed the problem as battery degradation linked to inevitable damage during servicing trips due in-part to the weight of the battery; one or two batteries even developed cracks and audible leaking. Additionally, fully discharging batteries each field session led to sulphation, or build up of lead sulphate in the batteries, lowering the batteries' ability to hold charge. Since we had to leapfrog the batteries, we were not able to bring faulty units back from the field to diagnose problems. To mitigate this problem, we ran "rejuvenation" modes on our battery chargers to break down sulphation, marked bad batteries to ensure they were preferentially rotated out of the network, and introduced a third battery at each site at the end of June, 2018. Despite these efforts, battery health gradually declined. Because field servicing intervals could not be reduced, data gaps of $>10$ days are visible before each servicing period throughout 2018. Additionally, New Zealand's lambing season in late 2018 meant we were not allowed access to land for up to two months. This is visible in the latter half of 2018 as a network-wide data gap. We were able to service the four sites that are recording during this gap, prior to the start of New Zealand's lambing season.

Probabilistic power spectral densities (PPSD) are a useful method for estimating the noise levels of seismic stations (McNamara and Boaz, 2006). PPSD plots were used to estimate relative noise levels of stations, and identify noisy stations that could be shifted to secondary locations (Figure 2.10). It was assumed that coastal sites would be noisier due to ocean noise from the secondary microseism (e.g. Friedrich et al., 1998). PPSD plots indicated a few candidates that showed high noise levels in the period range of interest (10-30 s), and so these stations were removed and reinstalled during subsequent field servicing (Figure 2.9). New sites were given new station codes, regardless of distance moved. For some sites, instruments were moved $<1 \mathrm{~km}(\mathrm{RD} 11 \rightarrow$ RD20, RD17 $\rightarrow$ RD21; Figure 2.11; Table 2.1), while others were relocated to completely new locations (RD19, RD22). This is noted in Figure 2.9 and Table 2.1.

One site was prematurely uninstalled during the November 2018 field servicing. Site RD14 (Pukenui) was"found in shambles" with equipment scattered around a 
$10 \mathrm{~m}$ radius, and power and data cables torn through. We conjectured that strong winds through the valley had blown the fish bin cover off and scattered the equipment, and that a curious animal had chewed through the cables. Since the network was to be dismantled early the next year (2019), a decision was made to not replace the equipment with available spares.

\subsubsection{Instrument response}

After each servicing, data were downloaded onto GNS servers using the Nanometrics software, Apollo $4^{4}$. The data were archived in SEED format ${ }^{5}$ (Standard for the Exchange of Earthquake Data). Time series data were stored in miniSEED format $t^{6}$ As standard in SEED, files were separated by day in coordinated universal time (UTC), and by channel (North-South, East-West, Vertical). Output data were sampled at $100 \mathrm{~Hz}(\mathrm{dt}=0.01 \mathrm{~s})$.

Instrument response files were created using the Nominal Response Library (NRL), which is IRIS's repository of recommended manufacturer response information? Initially the response files were created using IRIS's Portable Data Collection Center software (PDCC), which is a Java-based application for creating response files 8 At a later stage, response file creation was shifted to ObsPy's NRL client? Both PDCC and ObsPy access NRL, so the response is expected to be the same for a given set of inputs. Table 2.2 provides sensor and datalogger specifications used to generate response files, with specific keys provided by GeoNet. The sensor response files differ slightly between $30 \mathrm{~s}$ and $60 \mathrm{~s}$ instruments, but the datalogger keys are consistent throughout the network.

\subsubsection{Long-period data issues}

We observed that a number of the BEACON stations are devoid of long-period energy ( $\mathrm{T}>2 \mathrm{~s})$ in all three components. A number of attempts were made to find a root cause but ultimately no satisfactory solution was found. This has been identified as a sensor-specific issue. The consequence is that 12 of the 22 stations are

\footnotetext{
${ }^{4}$ https://www.nanometrics.ca/products/software/apollo-server-data-acquisition

${ }^{5}$ https://ds.iris.edu/ds/nodes/dmc/data/formats/seed/

${ }^{6}$ http:/ /ds.iris.edu/ds/nodes/dmc/data/formats/miniseed/

${ }^{7}$ http:/ /ds.iris.edu/NRL/

${ }^{8}$ https://ds.iris.edu/ds/nodes/dmc/software/downloads/pdcc/

${ }^{9}$ https://docs.obspy.org/master/packages/obspy.clients.nrl.html
} 
$2017.186-2017.365$

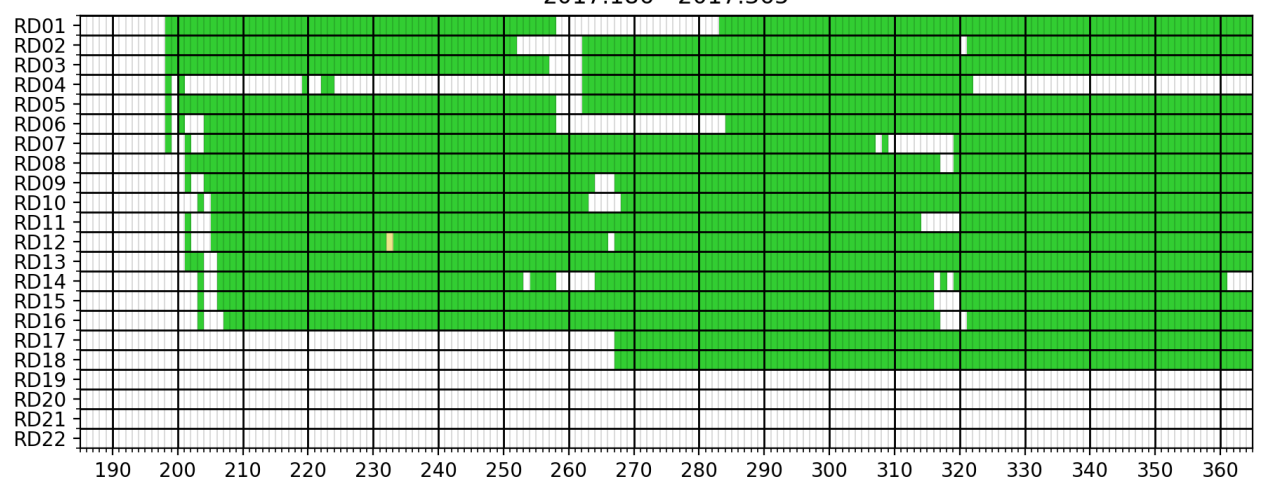

2018.001-2018.185
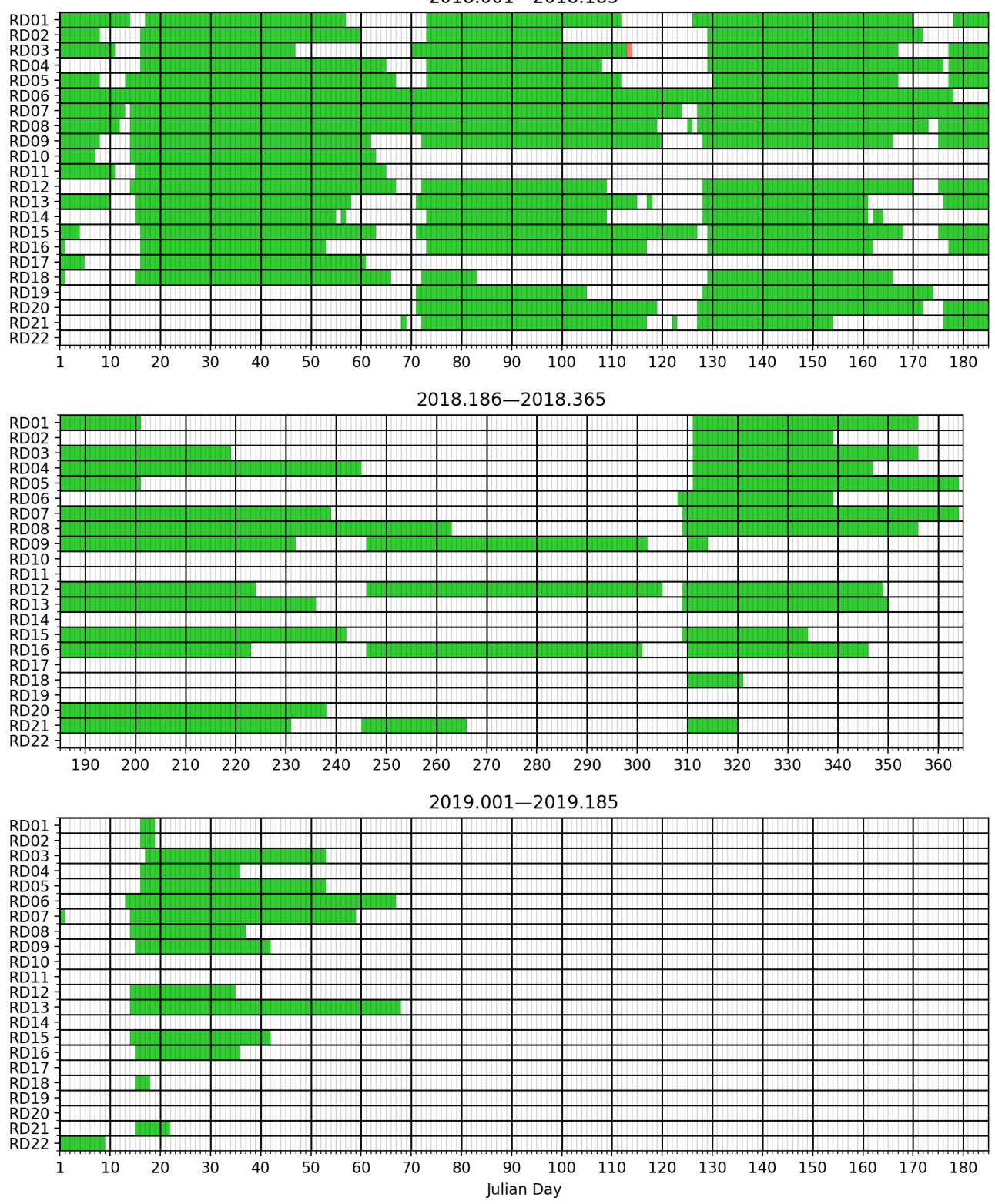

Figure 2.9: BEACON operations timeline. Data gaps visible at roughly 1-2 month intervals due to power loss prior to station servicing. An almost network-wide data gap is visible between 2018.230 and 2018.310, which coincided with the New Zealand lambing season. Green, yellow, and red blocks refer to days with only 3, 2, and 1 available component(s), respectively. 


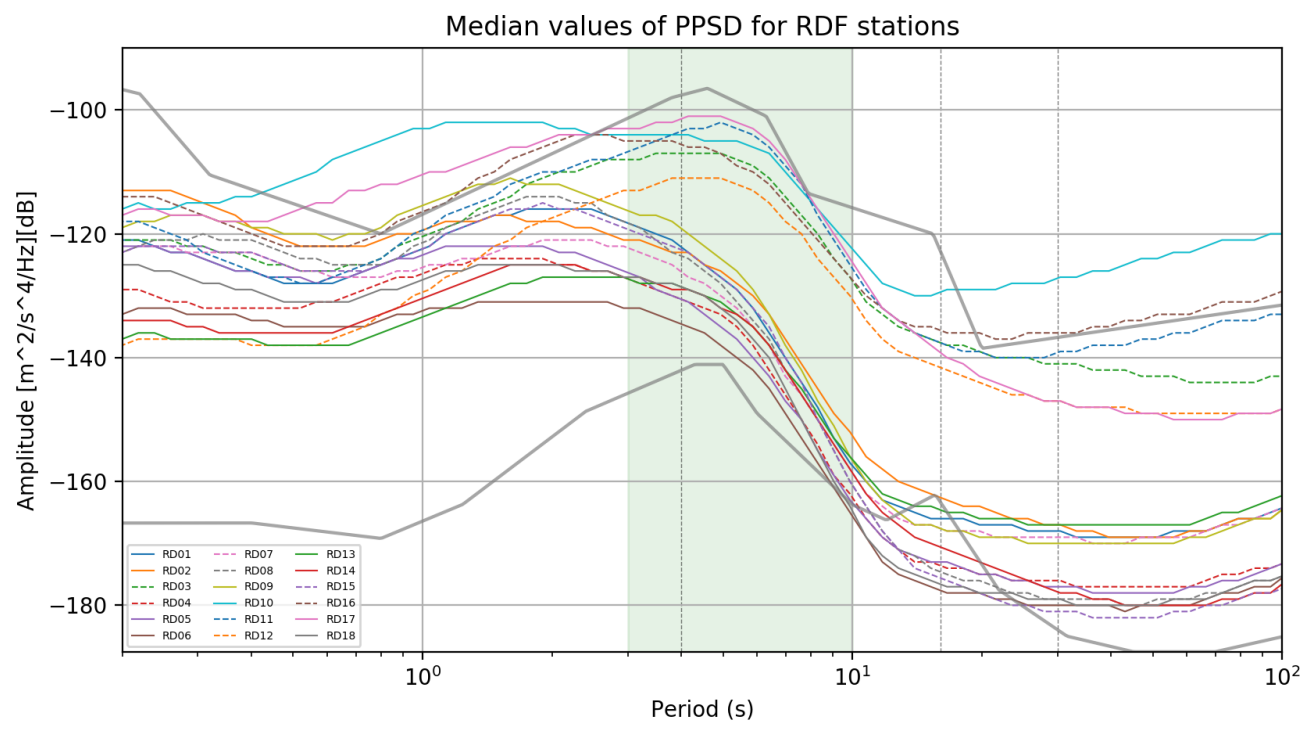

Figure 2.10: Probabilistic power spectral density (PPSD) plots for BEACON stations RD01-RD18. Gray lines represent the New Low Noise Model (bottom) and the New High Noise Model (top) (McNamara and Buland, 2004). Green shaded region shows the initial period range of interest for adjoint tomography (10-30 s). High noise at a subgroup of stations was used to identify candidate stations for relocations.

\begin{tabular}{|c|c||c|c|}
\hline Datalogger & Keys & Sensor & Keys \\
\hline \hline Manufacturer & Nanometrics & Manufacturer & Guralp \\
\hline Model & Tarus & Model & CMG-40T \\
\hline Input Range (Gain) & $16 \mathrm{Vpp}$ (1) & Natural Period (30s) & $30 \mathrm{~s}-50 \mathrm{~Hz}$ \\
\hline Input Impedance & Low (default) & Natural Period (60s) & $60 \mathrm{~s}-50 \mathrm{~Hz}$ \\
\hline DC Removal Filter & Off & Sensitivity & 800 \\
\hline Sample Rate (Hz) & 100 & - & - \\
\hline
\end{tabular}

Table 2.2: Nominal Response Library keys for BEACON sites. Datalogger keys are the same for all stations in the network. Sensor keys differ on the natural period of the sensor (Table 2.1) 
not usable for the tomographic efforts they were deployed to assist. Subsequent discussion with Emily Warren-Smith at GNS Science, who deployed this same network configuration in the southern alps (DWARFS) following the BEACON deployment, confirms this issue. We note that this issue is confined to long-period energy only, and high-frequency components are recorded nominally for all BEACON stations - the entire network can be used earthquake studies that do not rely on long-period waveforms.

During automated data-synthetic comparisons, we noticed a large percentage of BEACON stations were not returning any measurements for recorded earthquakes. Upon further inspection, it was observed that for the given passband (10-30 s) most BEACON stations were returning noise, with observed amplitudes 1-2 orders of magnitude smaller than synthetic amplitudes (Figure 2.11). This was surprising as the maximum data-synthetic amplitude differences for GeoNet stations were usually less than a factor of two. What was more surprising was that this problem was not pervasive through the network but seemed to affect stations at random. As seen in Figure 2.11A, station RD01 captures the earthquake in the raw waveform, with negligible differences to the raw waveforms from station RD10 (Figure 2.11C). However, when filtered for long periods (10-30 s), the amplitude on RD01 drops very close to the noise level (Figure 2.11B), while RD10 shows nominal recovery of long-period waves (Figure 2.11D).

The initial thought was that the issue lie in incorrect response files. However, changing any of the keys in Table 2.2 only served to decrease amplitudes. We sought to determine if this was an amplitude-only issue, or if it affected phase as well; if amplitude-only, we could find some acceptable scaling coefficient, because we are not concerned with amplitude information in our tomographic study. We analyzed large $\left(M_{\mathrm{w}}>7\right)$ teleseismic events with the idea that incoming teleseismic surface waves would arrive coherently across the network, removing ambiguity from near-source effects. Nearby GeoNet broadband stations were used as baseline for acceptable phase and amplitude. By looking at amplitude ratios we found a bimodal behavior in which some stations required scaling and others did not (Figure 2.12). This was used to determine which stations were effected (Table 2.1). Through this analysis, it was clear that certain instruments were not recording long-period energy (Figs. 2.10, C.1), and therefore any signal at long periods was 
suppressed well below the noise level; the incoming phase of seismic energy is convolved with the noise signal, ultimately leading to unusable data. At this point the decision was made that no additional time should be committed to this issue, and stations identified as being devoid of long-period energy were removed from our tomographic inversion (Table 2.1).

This issue affects roughly $60 \%$ of the BEACON dataset; in this thesis we use the remaining $40 \%$ of (good quality) waveform data from BEACON for the tomographic inversion described in Chapter 4 . 


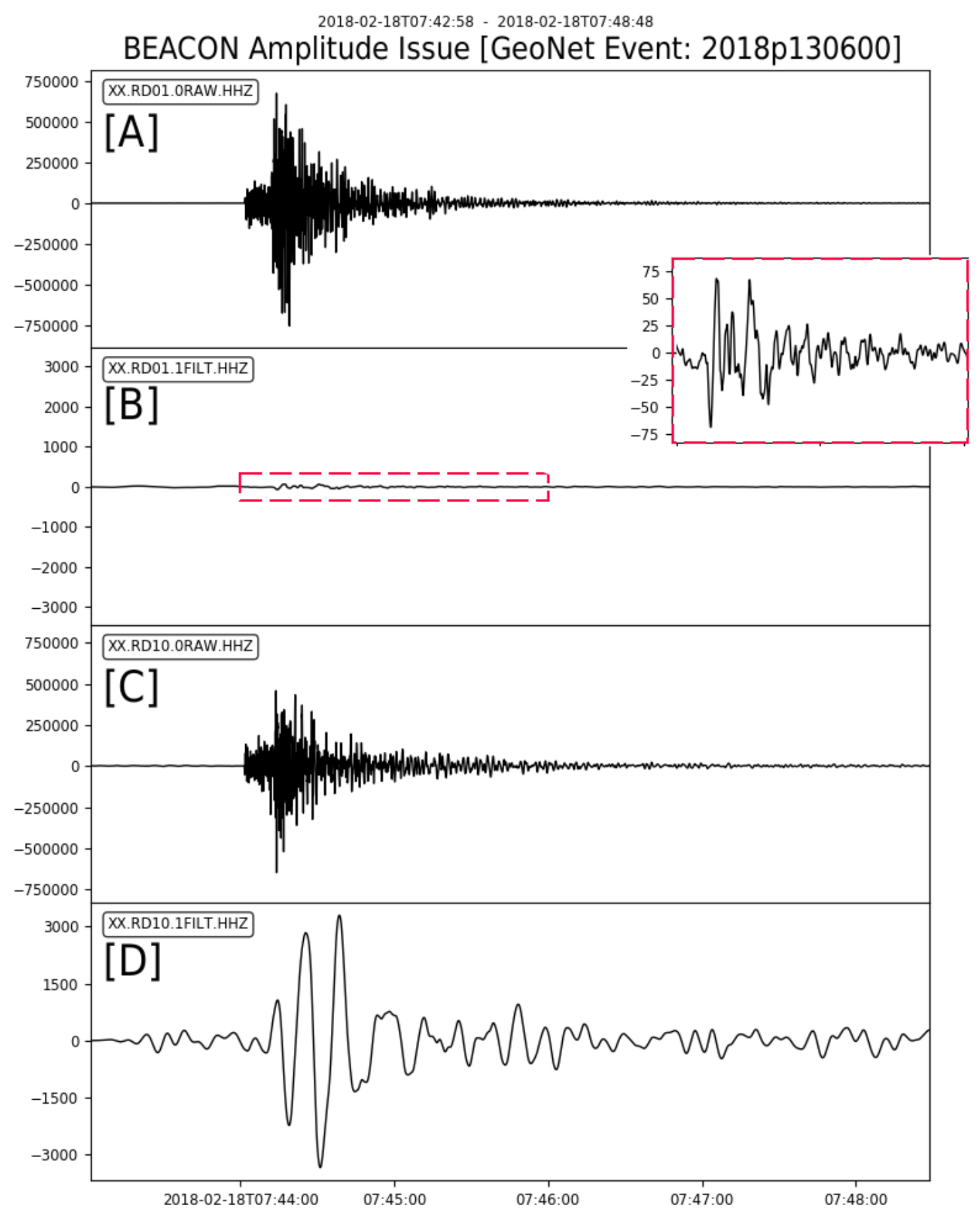

Figure 2.11: Example of BEACON long-period data issue for an earthquake in southern Hawke's Bay at 20 km depth (GeoNet ID: 2018p130600). A) Raw waveform data for the vertical component of RD01. B) RD01 waveform filtered at 10-30 s periods. Red dashed box corresponds to inset which shows earthquake arrival scaled to actual data bounds. C) Raw vertical component waveform data for RD10. D) RD10 waveform filtered at 10-30 s, identical processing as in (B). Units given in counts. 


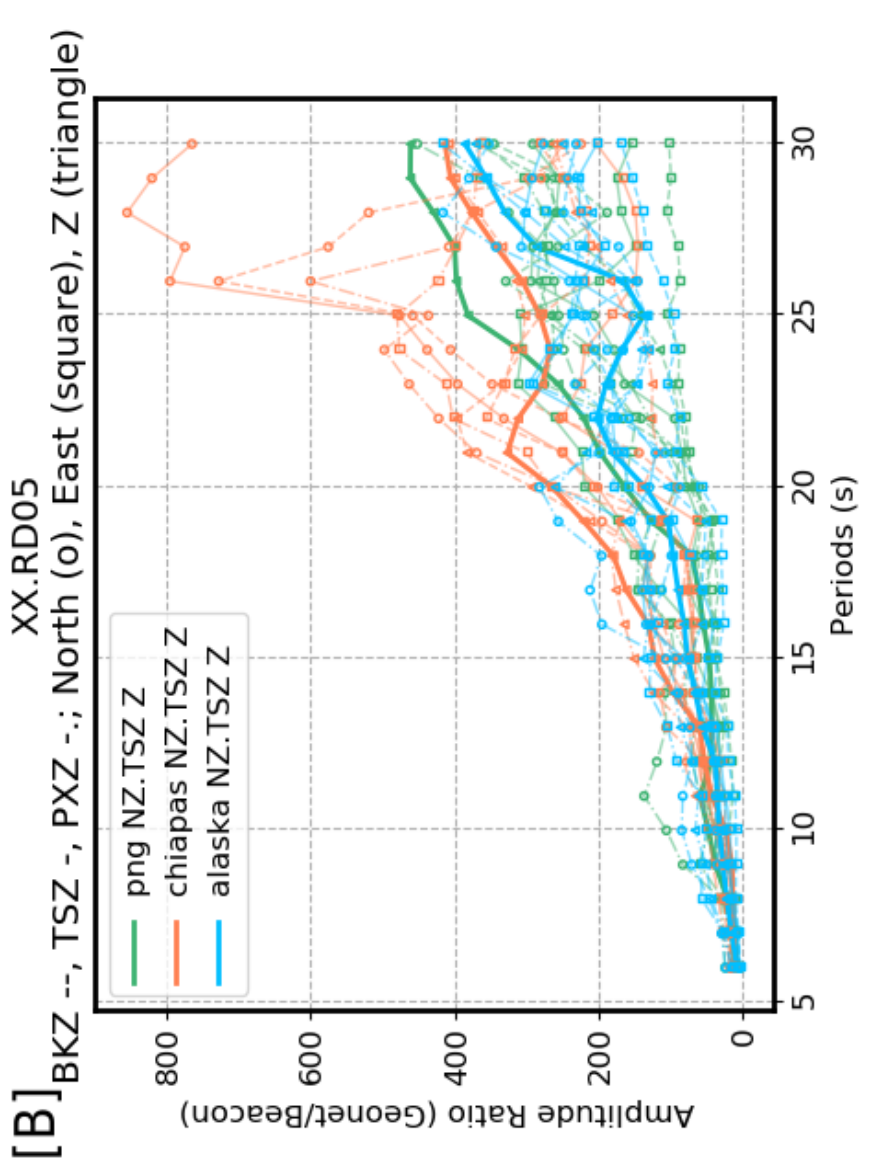

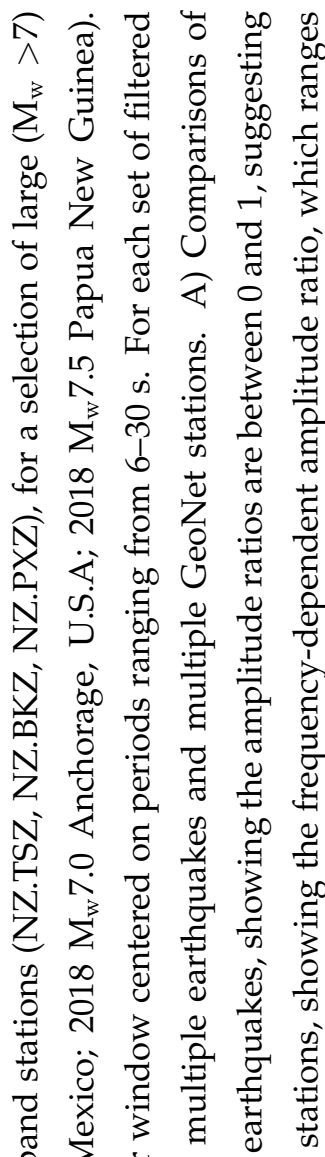
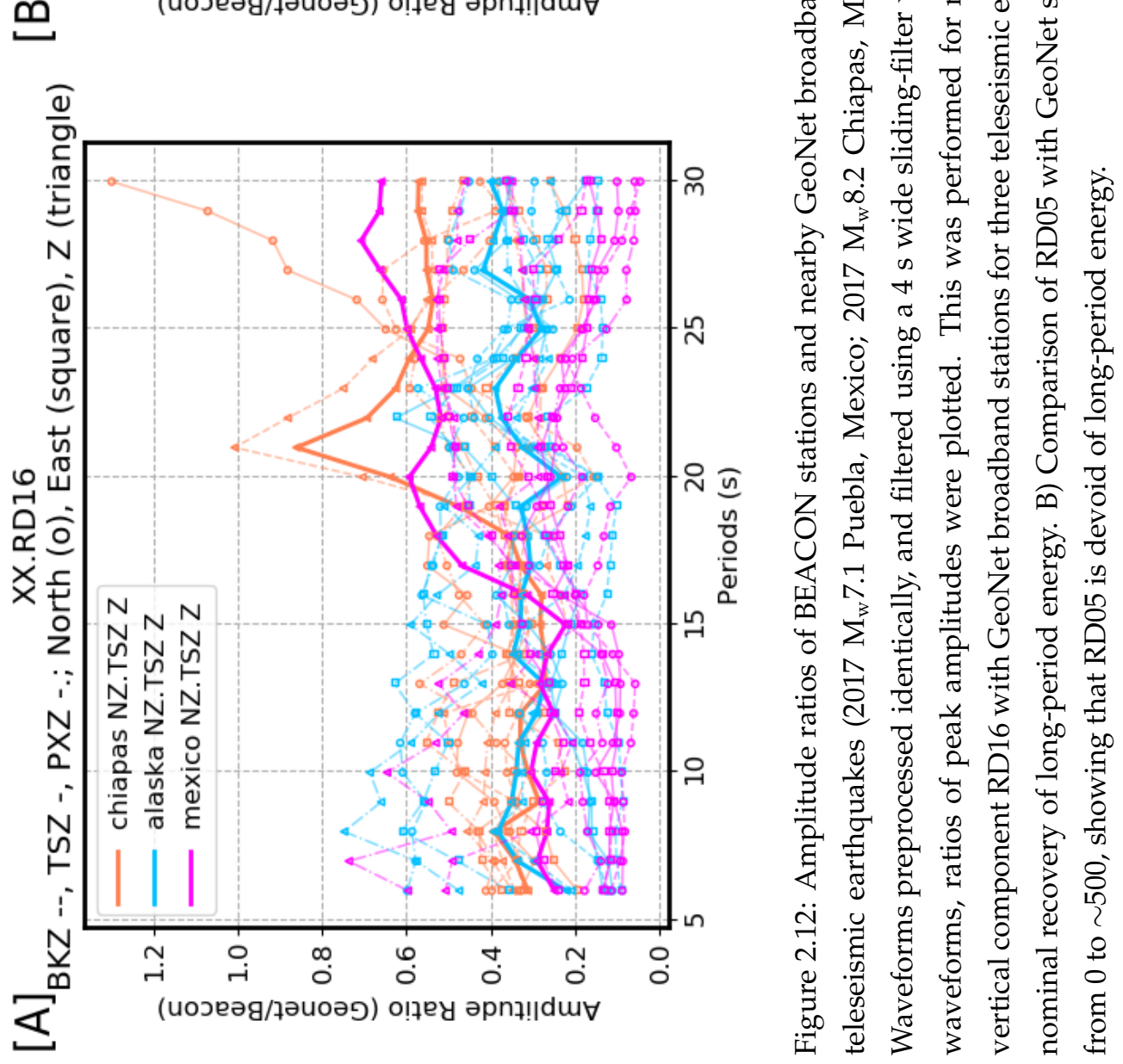


\section{Chapter 3}

\section{An automated workflow for adjoint tomography}

We develop and verify an automated workflow for full-waveform tomography based on spectral element and adjoint methods. We choose the North Island, New Zealand as a study area because of its high seismicity, extensive seismic network, and the availability of a candidate ray tomography starting model. To assess the accuracy of this model, we simulated 250 regional earthquakes using a spectral element solver, and compared the resulting synthetics with recorded waveforms. In a 10-30 s passband, reasonable cross-correlation phase and amplitude misfits exist between data and synthetics, whereas at 2-30 s, waveform misalignment is severe enough that meaningful cross-correlation measurements are no longer possible. To improve the velocity model at these short periods, we created an automated inversion framework based on existing tools for signal processing, phase measurement, nonlinear optimization, and workflow management. To verify the inversion framework, we performed a realistic synthetic inversion for 3D checkerboard structure and analyzed model recovery, misfit reduction, and waveform improvement. The results of this analysis show that the source-receiver distribution within the chosen domain is capable of resolving velocity anomalies in regions of sufficient data coverage, and of magnitudes comparable to those expected in a real

\footnotetext{
${ }^{1}$ Bryant Chow, Yoshihiro Kaneko, Carl Tape, Ryan Modrak, John Townend, An automated workflow for adjoint tomography-waveform misfits and synthetic inversions for the North Island, New Zealand, Geophysical Journal International, Volume 223, Issue 3, December 2020, Pages 1461-1480, https://doi.org/10.1093/gji/ggaa381
} 
seismic inversion. Along with this finding, the relative ease of use and reliability of the workflow motivates future efforts targeting a high-resolution (2-30 s), largescale ( $>50,000$ measurements) seismic inversion for the North Island. Updated models from such an inversion are expected to improve ground motion predictions, constrain complex velocity structures, and advance understanding of New Zealand tectonics.

\subsection{Introduction}

Seismic tomography offers a powerful tool for understanding the dynamics and evolution of our planet. Computational advances in the 2000s opened the door for advanced tomographic methods, including full-waveform modeling and inversion based on spectral element (Komatitsch and Vilotte, 1998; Komatitsch et al. 2002) and adjoint methods (Tarantola, 1984; Talagrand and Courtier, 1987; Luo and Schuster, 1991). Nevertheless, such inversions remain challenging not only in terms of computational cost, but also due to the complex software required to perform them.

Full-waveform adjoint tomography has led to detailed tomographic images from regional (e.g. Chen et al., 2007a; Tape et al., 2010; Lee et al., 2014b; Miyoshi et al. 2017), to continental (e.g. Fichtner et al., 2009, 2013; Zhu et al., 2015; Chen et al. 2015), to global scales (e.g. Bozdağ et al., 2016). In this study we define regional scale as domains spanning less than $10^{\circ}$. Inversions at continental scales and larger typically fit long-period waveforms (e.g. $>8 \mathrm{~s}$ as in Fichtner et al., 2013), require spherical meshes to honor Earth structure, and examine upper mantle and longwavelength crustal structure. In comparison, regional scale inversions typically employ Cartesian meshes and examine shallow crustal structure related to shortperiod waveforms ( $>2 \mathrm{~s}$ as in Tape et al., 2010). One constraint that limits the applicability of adjoint tomography is the assumption that the starting velocity model be close enough to true Earth structure to avoid spurious local minima in the inversion procedure. Assessments of candidate models, based on waveform fits, can be carried out to judge their suitability in this respect (Bao and Shen, 2016, Gao and Shen, 2015; Lee et al., 2014a; Lin et al., 2011; Taborda et al., 2016). Additionally, sufficient data coverage within the domain is required, as tomographic models from earthquake-based inversions can be spatially biased due to uneven 
distributions of sources and receivers. Ambient noise tomography (e.g. Shapiro et al., 2005; Lin et al., 2007, 2008; Yao et al., 2010; Rawlinson et al., 2016) can improve upon these difficulties, but typically limits shortest periods to $>5 s$ to capture surface wave dispersion. Therefore, to attain high-resolution images of shallow crustal structure and complex tectonic features in seismically active regions, it is crucial to develop modern tools to address the unique challenges presented by earthquake-based adjoint tomography.

The domain encompassed by the central and southern North Island and the northeastern South Island, New Zealand (Figure 3.1) is a prime candidate for performing regional-scale adjoint tomography. The domain exhibits high seismicity rates due to active subduction of the Pacific plate below the Australian plate (Reyners. 1998: Wallace et al., 2004, 2012b), in a region known as the Hikurangi subduction zone. The Hikurangi trench is directly offshore $(\sim 100 \mathrm{~km})$ and the plate interface below land is shallow, at roughly $15 \mathrm{~km}$ below the east coast of the North Island (Wallace et al., 2009; Barnes et al., 2010; Williams et al., 2013). Intraplate seismicity within the Pacific slab, interplate seismicity along the principal décollement, and upper plate seismicity associated with volcanism in the central North Island and strike-slip faulting along the length of the Hikurangi margin, collectively provide a geographically and kinematically diverse set of earthquake sources. More than a decade of earthquake moment tensors (Ristau, 2008) and broadband seismic data are available through the permanent seismic network of New Zealand (GeoNet). Additionally, many years of research by Eberhart-Phillips and colleagues have produced a detailed 3D velocity model (Eberhart-Phillips et al., 2010, 2014, 2015. 2017, 2020a; Eberhart-Phillips and Fry, 2017, 2018) that can be used to generate synthetic seismograms for the region. These qualities make this area a good choice for developing methods for adjoint tomography, and applying them to a region of complex structure and substantial seismic hazard (Stirling et al., 2012).

In this chapter, we present a methodological framework for full-waveform tomography using adjoint methods. The purpose of this work is three-fold:

1. To use 3D wavefield simulations to evaluate a candidate starting velocity model of New Zealand;

2. To expand the capabilities of an open-source automated workflow package for adjoint tomography (SeisFlows), by providing a modular interface for 


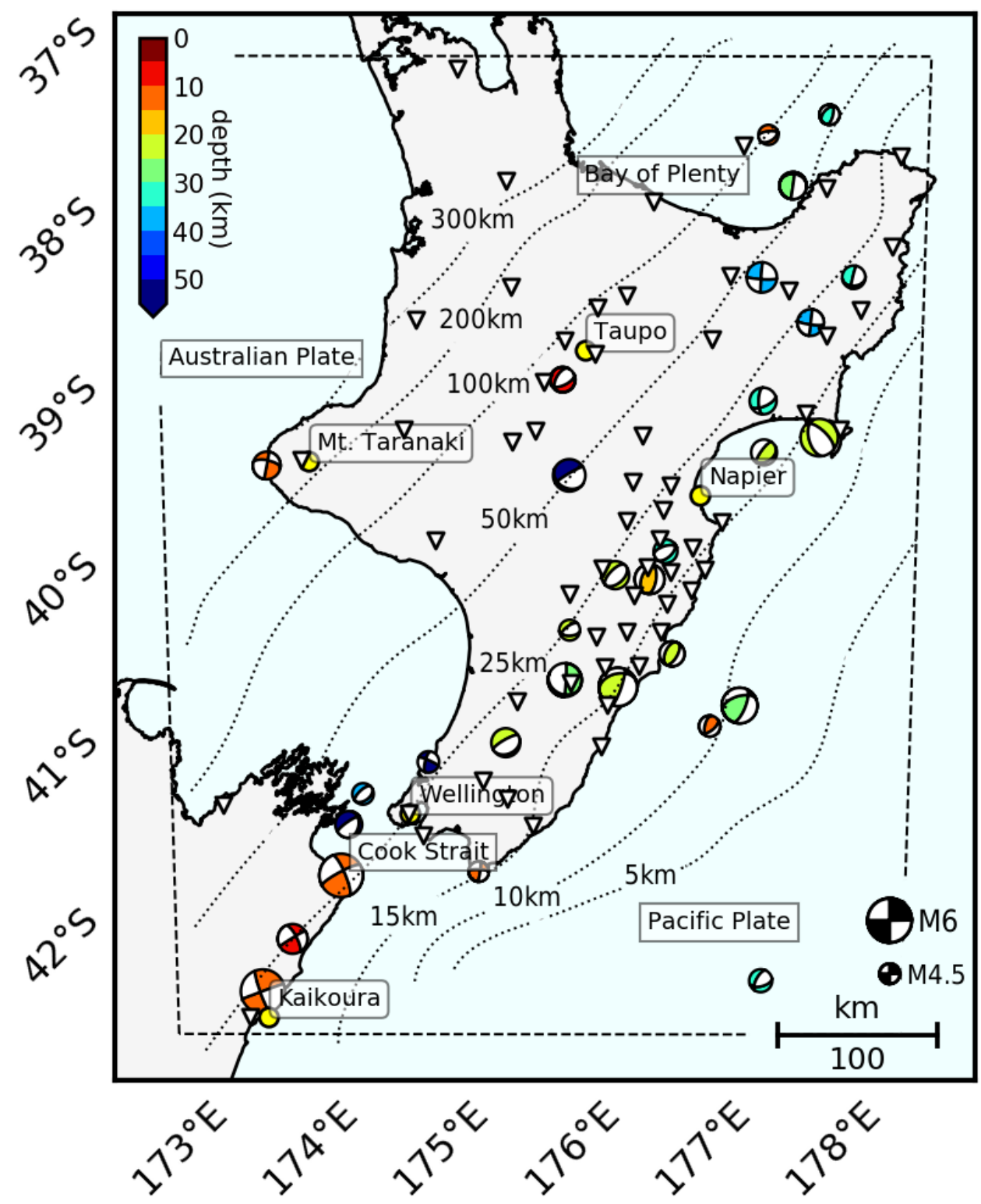

Figure 3.1: Map view of the study region, containing the central and southern North Island and the northeastern tip of the South Island, New Zealand. 30 earthquakes and 58 receivers used in synthetic inversions are shown as focal mechanisms and inverted triangles, respectively. Earthquakes are chosen to provide a varied representation of available focal mechanisms within the domain. Beachball size corresponds to magnitudes $4.8 \leq \mathrm{M}_{\mathrm{w}}<6$; depth information coded by color. The simulation domain is outlined by the dashed black line. Select cities or landmarks are shown as yellow circles. Named regions and tectonic plates are shown with square name plates. Dotted lines represent the approximate depth contours of the subduction interface, with depths denoted by inline annotations (Williams et al. 2013). 
misfit assessment (Pyatoa); and

3. To demonstrate the potential of this software suite for large-scale adjoint tomography through complete synthetic inversions using New Zealand station and earthquake distributions.

In developing an automated workflow, we aim to promote transparency and reproducibility in seismic tomography, and encourage community development through expansion of existing open-source software packages. We start with analysis of synthetics computed using the latest ray tomography model of New Zealand (Section 3.2). We then introduce and describe an automated inversion workflow to be used in current and future inversions (Section 3.3). This is followed by an application of the workflow to realistic synthetic inversions (Section 3.4), designed to illustrate the capability for resolution testing and assessment of methodological choices in large-scale inversions. The chapter concludes with a discussion looking toward full-scale adjoint tomography of the North Island, New Zealand, and applications elsewhere (Section 3.5).

\subsection{Misfit assessment of an initial 3D velocity model}

We evaluate a New Zealand velocity model to assess its applicability as a candidate starting model for adjoint tomography. Using ray-based body-wave traveltime tomography, Eberhart-Phillips et al. (2010) jointly computed earthquake hypocenters and seismic velocities $\left(\mathrm{V}_{p}, \mathrm{~V}_{p} / \mathrm{V}_{s}\right)$ in a New Zealand-wide threedimensional velocity model ("NZ-wide"). These velocity models incorporate traveltime measurements from local earthquake data and shots from active seismic experiments, recorded on permanent and temporary seismic stations throughout New Zealand. Portions of the model have since been improved through inversions for Rayleigh-wave group velocity maps (Eberhart-Phillips and Fry, 2017), and joint inversions of local earthquake and teleseismic data (Eberhart-Phillips and Fry, 2018). Attenuation models $\left(Q_{p}, Q_{s}\right)$ have also been constructed by fitting spectral decay for body waves from local earthquakes (Eberhart-Phillips et al., 2014, 2015, 2017, 2020a).

In this study, we use a portion of the NZ-wide velocity model, which we refer to as "NZ-North". Figure 3.2 shows map views and cross sections of the NZ-North 
$\mathrm{V}_{p}$ and $\mathrm{V}_{s}$ models. Tectonic features of the North Island and the Hikurangi subduction interface (Wallace et al., 2004, 2009; Clark et al., 2019) are identifiable by the stark velocity contrasts. The lowest velocities within the model are found in the accretionary prism of the subduction forearc, which is akin to a sedimentary basin, shown to exhibit extremely long durations of ground motion following seismic events (Kaneko et al., 2019). In the central North Island, the modern (2 Myr) portion of the volcanic arc, the Taupō Volcanic Zone, exhibits high heat flow and geothermal activity, extensional faulting, and associated seismicity, underlain by low-velocity, highly attenuating crust and mantle (Wilson et al., 1995, 2009; Behr et al. 2011; Rowland et al., 2010; Eberhart-Phillips et al., 2020a). Cross sections show the subducting Pacific plate and overlying Australian plate, as well as low velocity sediments in the forearc region. The northeastern extent of the South Island is included in this domain, as seismicity there is high in the southward transition of the subduction margin from oblique convergence to strike-slip behavior. The southwest corner of NZ-North contains the $2016 \mathrm{M}_{\mathrm{w}} 7.8$ Kaikōura earthquake (Hamling et al., 2017; Holden et al., 2017) and its extensive aftershock sequence.

\subsubsection{Simulation framework}

We use the NZ-North velocity model to carry out spectral element wave propagation simulations to assess its applicability for adjoint tomography based on waveform fits. To generate synthetic seismograms, we use the spectral element solver SPECFEM3D Cartesian (Komatitsch and Tromp, 2002a b) to simulate ground displacement from local earthquakes. Using the SPECFEM3D internal mesher, a rectangular mesh was created with roughly 600000 elements and a minimum element spacing of $1 \mathrm{~km}$. Topography and bathymetry, necessary for accurate propagation of surface waves, are interpolated from SRTM-30P (Becker et al., 2009). A water layer is not used in this mesh. Mesh boundaries are chosen to maximize the number of local earthquakes within the domain, while limiting unused domain space. A mesh depth of $400 \mathrm{~km}$ is chosen to avoid numerical reflections from the bottom boundary.

Our earthquake catalog consists of 250 moment tensors from local earthquakes of moment magnitudes $4.4 \leq \mathrm{M}_{\mathrm{w}}<6.0$ that occurred between January 2005 and November 2019 at depths shallower than $60 \mathrm{~km}$. The depth and lower magnitude bound are chosen to discard events that will not excite strong surface wave 

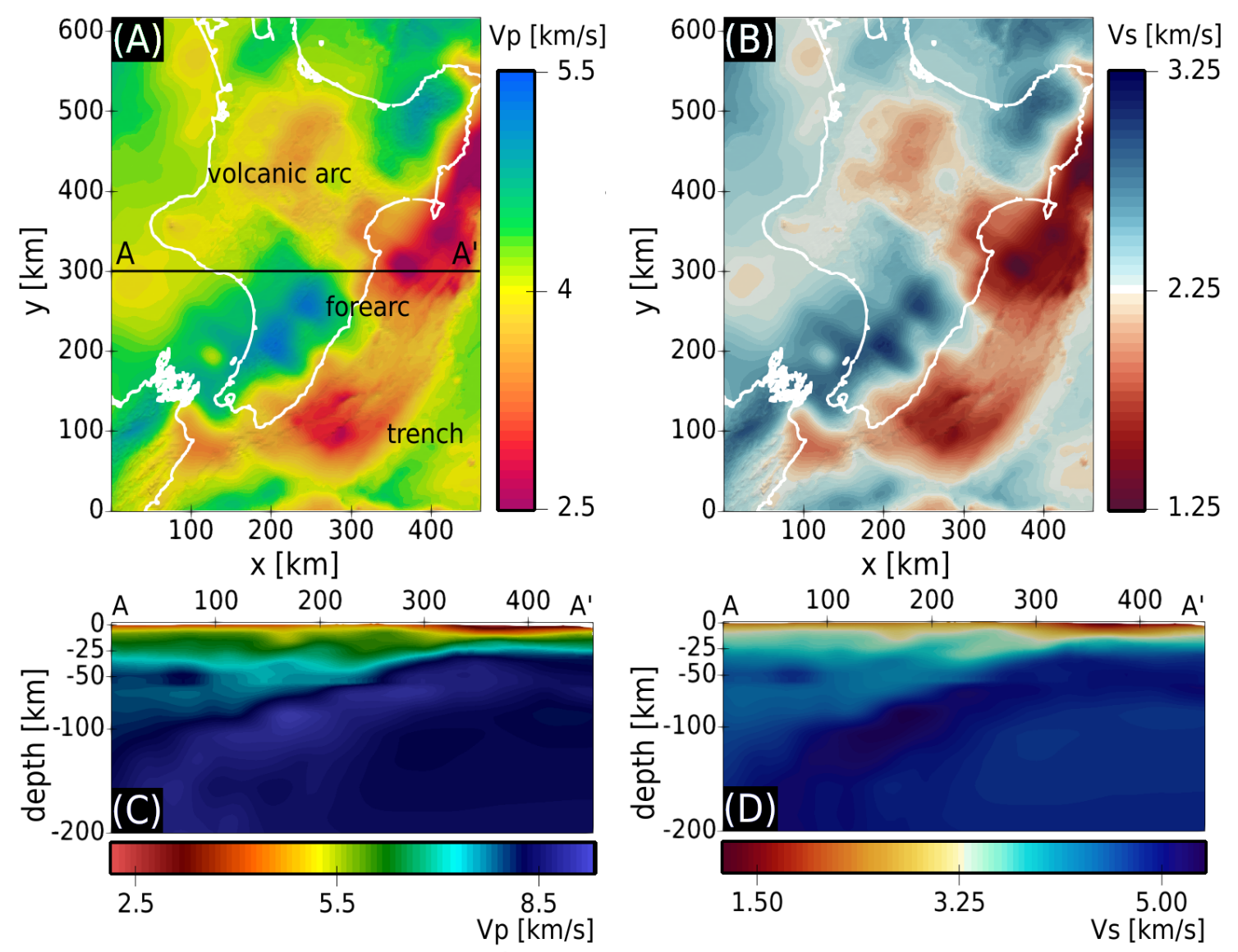

Figure 3.2: A) Map view of the NZ-North $\mathrm{V}_{p}$ model. Coastline shown as white line. Line $\mathrm{A}-\mathrm{A}^{\prime}$ corresponds to the surface trace of cross sections in (C-D). Text labels provide general locations of Hikurangi subduction zone features. B) Map view of the NZ-North $\mathrm{V}_{s}$ model. C-D) Cross sections of the $\mathrm{V}_{p}$ and $\mathrm{V}_{s}$ models, corresponding to the line A- $\mathrm{A}^{\prime}$ in $(\mathrm{A})$. 
signals, while the upper magnitude bound is set to avoid the need for finite-fault source representations. Moment tensors are routinely calculated by GeoNet for the New Zealand region (Ristau, 2008, 2013) using a time domain moment tensor inversion code (Dreger, 2003) and New Zealand-specific 1D velocity models. These regional moment tensors have been shown to compare well with Global CMT solutions (Ekström et al., 2012) and first motion focal mechanisms (Robinson and Webb, 1996). We calculate synthetic seismograms at locations corresponding to 45 GeoNet broadband stations.

\subsubsection{Misfit assessment}

We assess the degree to which the NZ-North velocity model generates synthetic seismograms which accurately reproduce seismic observations. We do this by performing forward simulations for all events in our catalog and quantifying waveform accuracy using a time windowing algorithm, which compares similar sections of waveforms and excludes noise or poor-fitting data. Here, time windows are chosen using the FLEXWIN algorithm (Maggi et al., 2009), which identifies wave-group like signals using a short-term average/long-term average (STA/LTA) ratio. The selection algorithm identifies acceptable time windows based on waveform fit, characterized by peak cross-correlation, time shift, and amplitude ratio. Amplitude ratio is defined by Dahlen and Baig (2002) as

$$
\Delta \ln (A)=\ln \left(\frac{A_{\text {obs }}}{A_{\text {syn }}}\right)=0.5 \ln \left[\frac{\int d^{2}(t) d t}{\int s^{2}(t) d t}\right],
$$

where $d$ and $s$ are the data and synthetic waveforms, respectively. User-defined parameters allow control over the quality of waveforms contained within the chosen windows. For this region, we find adequate window selection when absolute time shift does not exceed $8 \mathrm{~s}$, absolute amplitude ratio is less than 2, and peak cross-correlation is greater than 0.7. For reference, a window around two identical waveforms has time shift and amplitude ratio equal to 0 , and peak crosscorrelation equal to 1 . A summary of windowing parameters used in this study is provided in Table A.1.

\section{Selected waveforms}

Here we show a representative set of average and below-average vertical-component waveforms from various source-receiver paths to provide a sense of the varying 
degrees of misfit for the candidate velocity model. Waveforms are shown for a period band of 10-30 s (Figure 3.3 $\mathrm{A}-\mathrm{C}$ ) and 2-30 s (Figure 3.3 $\mathrm{A}^{\prime}-\mathrm{C}^{\prime}$ ), corresponding to the labelled source-receiver paths in Figure 3.3D. Window annotations provide a reference for misfit through values of time shift (dT), amplitude ratio (dA), and peak cross-correlation (cc). By convention, a positive time shift corresponds to synthetic phases arriving early with respect to observed phases $\left(\mathrm{dT}=T_{\mathrm{obs}}-T_{\mathrm{syn}}\right)$, and a positive amplitude ratio corresponds to synthetic amplitudes that are smaller than observed amplitudes.

In Figure 3.3. waveform A at 10-30 s represents average misfit. Cross-correlation is high for both misfit windows, however time shift is large for the later window. At 2-30 s periods, a stark increase in misfit is illustrated by low cross-correlation values and no selected windows around the later arriving phases. This average misfit may be explained by the raypath crossing an area of minimal data coverage. Waveform B of Figure 3.3 traverses the Taupō Volcanic Zone, characterized by average time shift but large amplitude difference at 10-30 s periods. At 2-30 s periods, waveform peak amplitudes are more than a factor 10 different, contributing to a short selected time window. This large amplitude difference may be due to inaccuracies in the attenuation model for this complex volcanic region. Waveform C of Figure 3.3 traverses parallel to the Hikurangi forearc region. Observations of surface waves in this area show increased durations of ground motions due to the basin-like resonance effects of the low-velocity accretionary prism. Inaccuracy in the initial velocity model is observable in both period bands, where synthetic seismograms do not fully capture the resonant waveforms shown in the observations.

The same analysis for radial and transverse components (Figure D.3) show similar waveform behavior and window selections. Synthetics are also generated using a North-Island-specific 1D velocity model (Ristau, 2008). Comparisons between the resultant synthetic seismograms (Figure D.1) show that NZ-North is more capable of capturing accurate waveform fits and travel times, especially for later arrivals. Although waveform amplitudes are better fit with the 1D velocity model of Figure D.1, this is deemed less important due to our intended use of phase-based objective functions (Section 3.4.3). 
$10-30 \mathrm{~s}$
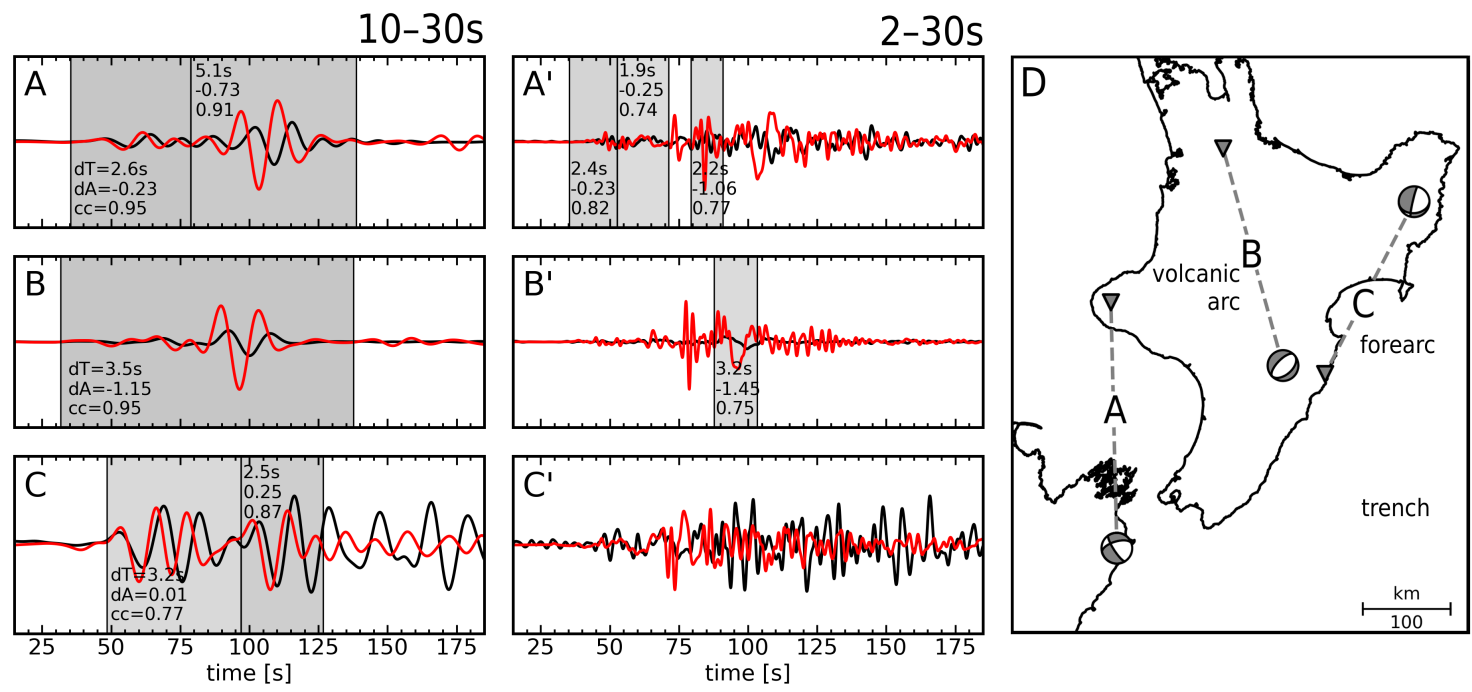

Figure 3.3: A representative selection of vertical-component data-synthetic comparisons. A-C) Misfit comparisons for the 10-30 s period band. Observed waveforms are shown in black, synthetic waveforms in red. The gray boxes represent misfit windows chosen by Pyflex. Measured time shift (dT), amplitude ratio (dA), and peak cross-correlation (cc) are indicated within each window. Waveforms are shown in units of displacement. $\mathrm{A}^{\prime}-\mathrm{C}^{\prime}$ ) The same waveforms as (A-C), but filtered at a period band of 2-30 s, and windows chosen with slightly altered windowing parameters (Table A.1. D) Map of source-receiver locations with raypaths, focal mechanisms, and labels corresponding to general subduction zone features. 


\section{Bulk misfit analysis}

We analyze misfit in bulk for all collected measurements at a period band of 10$30 \mathrm{~s}$. Misfit histograms detail time shift, amplitude ratios, peak cross-correlation, and relative start time for 250 events recorded on three components of 45 broadband stations, with a total of 26676 measurements.

Time shifts (Figure 3.4A) show that, on average, synthetic phases arrive $2.0 \pm 3.65 \mathrm{~s}$ earlier than observed phases, meaning the model velocities are slightly fast with respect to observations. Amplitude ratios (Figure $3.4 \mathrm{~B}$ ) show a mean and standard deviation of $-0.07 \pm 0.68$, meaning that synthetic amplitudes are approximately $7 \%$ larger than observed amplitudes. Peak cross correlation (Figure 3.4C) shows a roughly uniform distribution of waveform fits up to a value of 0.85 , with progressively lower counts above that. Figures 3.4D-F show time shifts for vertical, radial, and transverse components, respectively. Radial and vertical components show a comparable number of measurements and average time shift, while a lower number and larger average time shift for the transverse component suggest less accurate synthetics for transverse-sensitive phases like $\mathrm{SH}$ and Love waves.

Figures $3.4 \mathrm{G}-\mathrm{I}$ relate to the relative start time of measurement windows with respect to the event origin time. The median start time is $86 \mathrm{~s}$, which suggests that a majority of measurements are made for direct arrivals and small source-receiver distances. Cross correlation values for measurements made before $100 \mathrm{~s}$ start time show a relatively uniform distribution, while measurements made after $100 \mathrm{~s}$ show progressively lower counts for cross-correlation values greater than 0.8 . Phases associated with later arrivals, i.e. surface waves and large source-receiver distances, show increased misfit, which can be interpreted either as accumulation of misfit for large propagation distances, or high misfit for surface waves stemming from a candidate velocity model primarily derived using body waves.

The overall low time shifts and amplitude ratios, high peak cross correlation values, and even distribution of measurements among components, suggest that NZNorth is a reasonable starting model for adjoint tomography. This is further evidenced through comparisons with bulk misfit analysis of synthetics generated using a 1D North Island velocity model (Figure D.2). Despite less restrictive windowing parameters for the 1D synthetics (Table A.1), only 9834 measurements could be made, with considerably larger average time shifts $(6.11 \pm 5.03 \mathrm{~s})$, and 
amplitude ratios $(0.55 \pm 0.73)$, illustrating significant improvements gained using the region-specific 3D velocity model. Additionally, analysis of the ray-based 3D starting model of southern California by Tape et al. (2010) show comparable overall time shifts $(1.06 \pm 1.43 \mathrm{~s})$ and amplitude ratios $(0.34 \pm 0.39)$ for 36553 measurements at 6-30 s periods. We argue that the larger average misfit for NZ-North is attributed more to the complicated nature of New Zealand tectonics with respect to southern California, rather than any substantial differences in accuracy for their respective starting models. The improvements in waveform fit seen in Tape et al., as well as the accuracy gained using NZ-North, in contrast to a 1D velocity model, provides optimism that this is a reasonable candidate starting model for adjoint tomography.

\subsection{Automated inversion workflow}

Adjoint tomography consists of many near-identical processes manipulating large amounts of data in an iterative procedure. It therefore benefits strongly from automation, parallelization, and structured organization. Here we present our workflow, which is comprised of new and existing open-source tools that address and automate each step of an earthquake-based adjoint tomography workflow. In this section we briefly outline the components of a typical workflow (Figure 3.5), followed by explanations of the individual software packages comprising our automated workflow.

Adjoint tomography typically begins with data gathering, characterized by selection of a region and collection of seismic data consisting of moment tensors, waveforms, and source and receiver metadata. An initial velocity model is generated for the region using external information, and the forward problem is solved by simulating seismic wave propagation for each source. The resultant synthetic waveforms are compared to corresponding seismic data through some objective function, in a misfit quantification procedure, or function evaluation. Here, time windowing can be used to restrict the data evaluated in the objective function, preferable as data quality can not always be well controlled in an automated framework. To solve the inverse problem, we seek to minimize the objective function through perturbations of either velocity model or seismic source parameters. This is accomplished by determining the gradient of the objective function, with respect to 

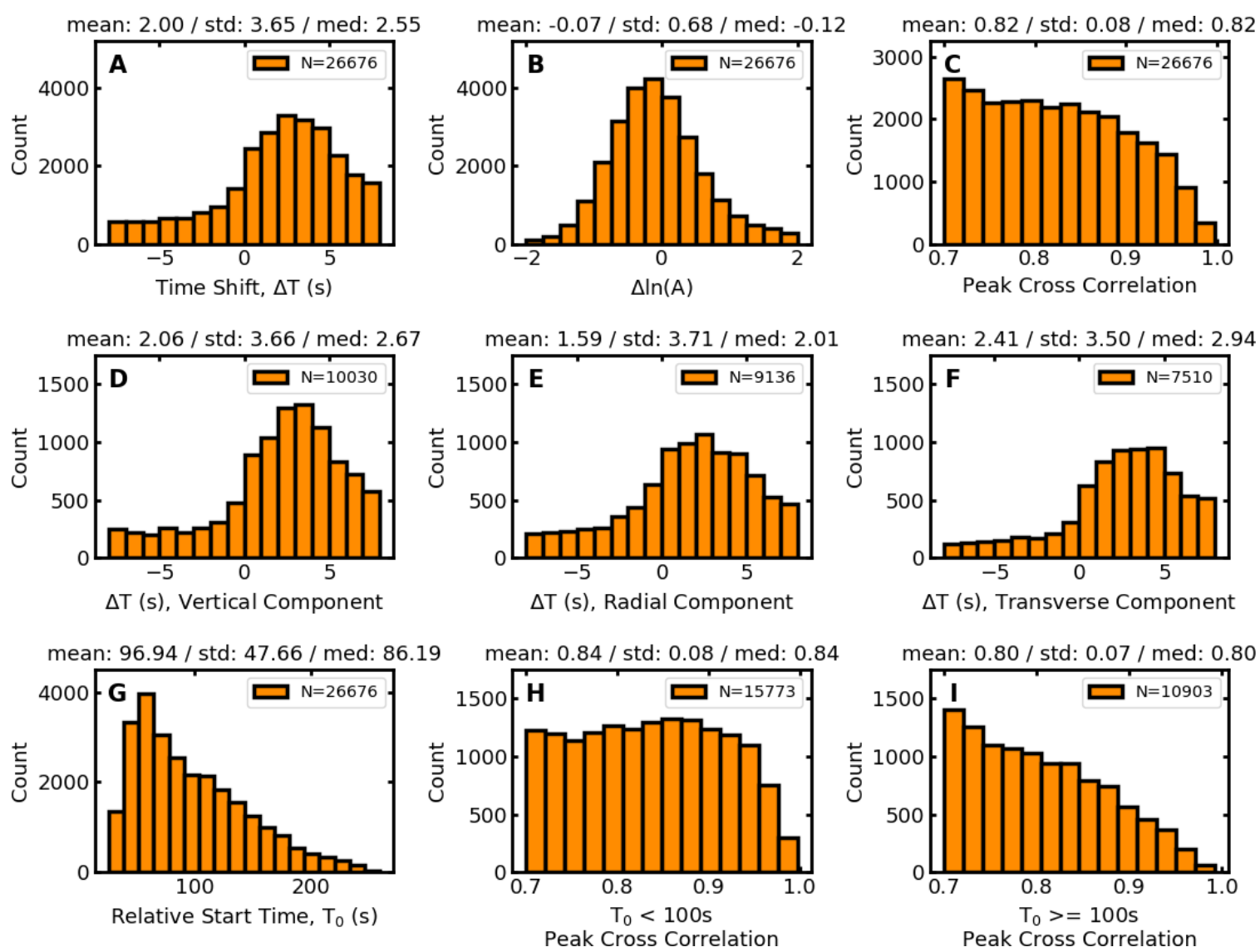

Figure 3.4: Misfit histograms for forward simulations using the NZ-North velocity model. Measurements from 250 events recorded on three components of 45 broadband stations, with 26676 total measurements. Mean, median, and standard deviation are given in the titles of each histogram. The number of measurements for each histogram is provided in the respective legend. A) Time shift corresponding to peak cross correlation between data and synthetics, in units of seconds. B) Data-synthetic amplitude difference $\Delta \ln (A)$ (Equation 3.1). C) Peak cross correlation value. D-F) Time shift for vertical, radial, and transverse component measurements. G) Measurement start time relative to event origin time. H) Peak cross correlations for measurements made before $100 \mathrm{~s}$ relative start time. I) Peak cross correlations for measurements made at or after $100 \mathrm{~s}$ relative start time. 


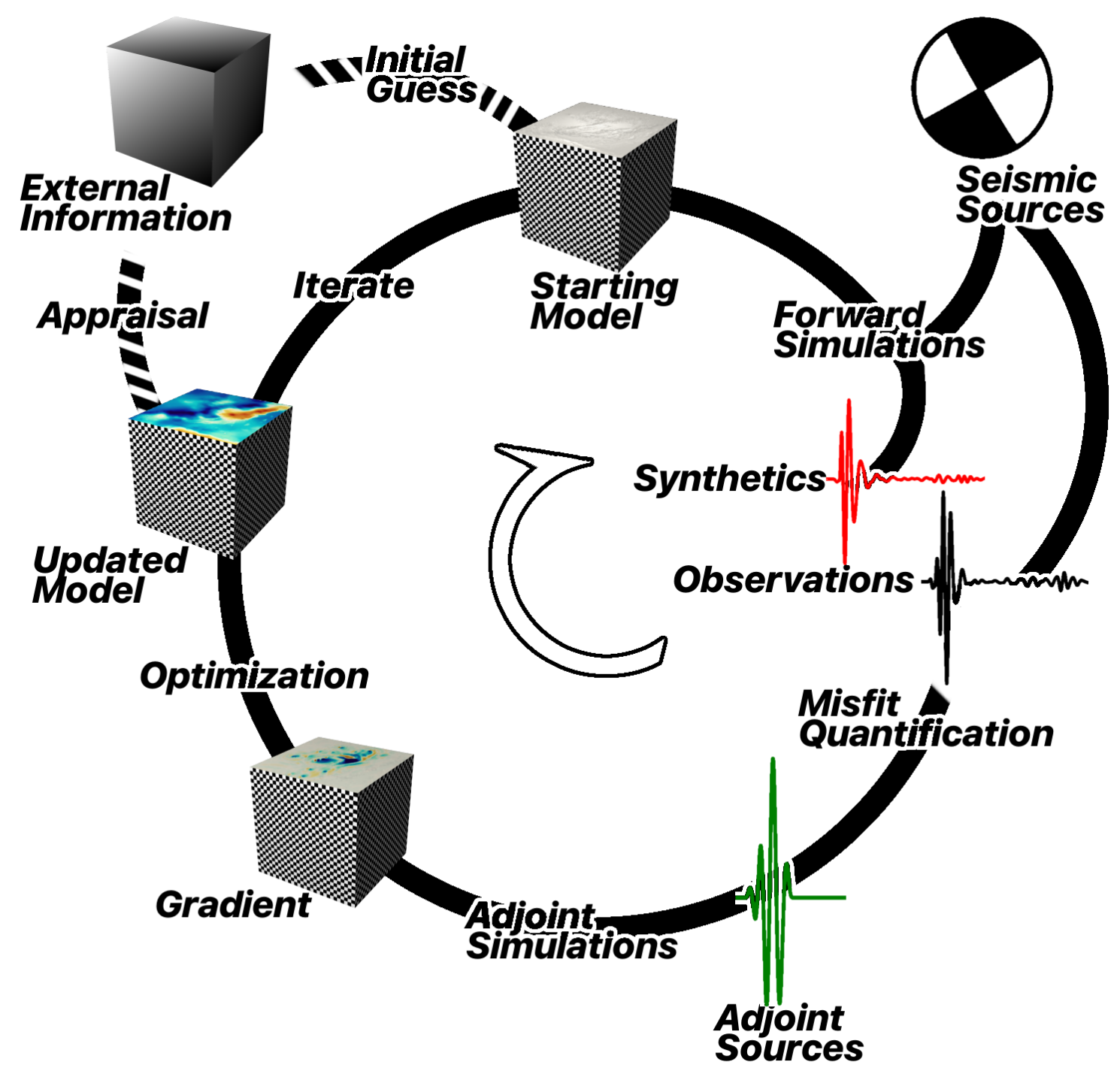

Figure 3.5: A diagrammatic illustration of the adjoint tomography workflow, adapted from Figure 2 of Liu and $\mathrm{Gu}$ (2012). The workflow tools introduced in this chapter are capable of automating all components of the adjoint tomography workflow, except for the initial guess, creation of the starting model, and the appraisal of the final model using external information. Inversions can be run fully-automated, or with various levels of manual intervention introduced throughout the inversion. 
these parameters, using the adjoint-state method (Tarantola, 1984). In the adjoint simulation, the forward wavefield interacts with a secondary (adjoint) wavefield to produce the gradient of the objective function for a given source-receiver pair. This adjoint wavefield is produced by an adjoint source, which is a source time function, input at receiver locations, weighted by misfit for a given source-receiver pair. Using gradient information from many source-receiver pairs, a nonlinear optimization algorithm searches for a local minima in the objective function through perturbations of model or source parameters. The forward problem is solved again with the perturbed model or source and evaluated for misfit reduction. This process repeats iteratively until some pre-defined convergence criteria are met. Updated models can be appraised through resolution tests, and comparisons with external information such as known geology, tectonics, or tomographic models. For more detailed explanations of adjoint tomography, see e.g. Tromp et al. (2005); Tape et al. (2007, 2010); Fichtner et al. (2009); Liu and Gu (2012).

Our aim in automating the adjoint tomography workflow is to increase the speed and efficiency with which an inversion can be initialized and performed, while thereby reducing the possibility of user error. We emphasize open-source code development to promote scientific reproducibility and increased transparency in seismic inversions. Desirable aspects of such a workflow include having as few programming languages as possible, the ability to implement varying degrees of manual control, and a flexible design that can be applied to a wide range of scales and systems. In the following sections we describe our chosen workflow tool, SeisFlows, as well as its complement package for earthquake tomography, Pyatoa.

\subsubsection{SeisFlows}

For automation of the adjoint tomography workflow, we employ SeisFlows (Modrak et al., 2018), a flexible waveform inversion package written in the Python programming language. SeisFlows provides custom routines for seismic inversions with modules that address, for example, workflow management, compute system interactions, and nonlinear optimization. Additionally, it integrates the functionalities of external numerical solvers, like SPECFEM3D Cartesian, using generalized wrapper functions. SeisFlows automates all components of a seismic inversion, from job submissions on high performance computing (HPC) systems to model updates, and has been used for active source full-waveform inversions (e.g. Smith 
et al. 2019) and synthetic inversion studies (e.g. Rusmanugroho et al., 2017; Matharu and Sacchi, 2018; Borisov et al., 2018; Tromp and Bachmann, 2019a b). SeisFlows has yet to be used for earthquake-based inversions, and therefore important aspects of earthquake tomography, such as seismic data handling and detailed misfit quantification, are underdeveloped with respect to the package's more robust components. This has led to the development of Pyatoa, a complementary package to enhance SeisFlows' capabilities for earthquake-based adjoint tomography.

\subsubsection{Pyatoa}

We have developed Python's Adjoint Tomography Operations Assistant (Pyatoa), an open-source tool designed to handle seismic data within a seismic inversion workflow. It primarily addresses the misfit quantification procedure, through gathering and processing of seismic data, and evaluation of objective functions. Pyatoa is built around the widely-used seismological processing package, ObsPy (Beyreuther et al., 2010; Krischer et al., 2015b), and benefits from the seismic data handling and gathering capabilities contained therein. Using custom methods and data structures, Pyatoa abstracts, or simplifies, routines defined by ObsPy and other existing Python packages to facilitate automation of misfit evaluation in a seismic inversion. Pyatoa additionally provides a highly-structured data organization scheme, and easy-to-use measurement aggregation tool, to allow for efficient storage, retrieval, and assessment of inversion data. The aim in developing Pyatoa is to codify the typically custom-made, hard-coded subroutines that are accessory, but nonetheless essential, when performing earthquake-based adjoint tomography. We provide a general explanation of the structure and functionalities of Pyatoa here, and leave a more technical explanation for the documentation 2 . A flowchart detailing the role of Pyatoa within a SeisFlows workflow is given in Figure D.4.

\section{Structured data storage}

Data gathered at each function evaluation are stored in custom file formats defined by the Adaptable Seismic Data Format (ASDF, Krischer et al., 2016). ASDF data sets are structured file formats built to store large amounts of seismic data.

\footnotetext{
${ }^{2}$ github.com/bch0w/pyatoa
} 
The use of data sets removes the need for cumbersome and error-prone directory structures commonly used in inversion workflows. This facilitates storage, transport, and analysis of inversion data, and increases the potential for reproducible seismic inversions. ASDF data sets are self-describing, as they contain both data and metadata. For Pyatoa, this means data sets provide all necessary information to understand and repeat previous function evaluations. ASDF data sets are built to store seismic waveforms and metadata as ObsPy objects, removing the need for data conversion in the transition from storage to processing. Pyatoa provides additional functionality to ASDF, allowing for storage of misfit windows, adjoint sources, and configuration parameters. Some pertinent applications of this added functionality: re-using previously derived misfit windows in a new function evaluation, or quickly re-evaluating misfit using new windowing parameters or objective functions.

\section{Misfit quantification}

At each function evaluation and for each source-receiver pair, observations and synthetics must be standardized, preprocessed, cut into time windows, and evaluated using an objective function. Pyatoa streamlines and automates these operations through custom workflow and data structures. Figure 3.6 shows an example end product of a Pyatoa function evaluation. Here we refer to standardization as the process of equating two time series, for example by start time and sampling rate, while preprocessing refers primarily to instrument response removal and frequency domain filtering. Through a custom data object which relates matching waveforms and metadata, Pyatoa wraps the processing functionalities of ObsPy to automatically standardize and preprocess related waveform data. A series of internal checks ensure that inappropriate waveforms are not included in the function evaluation, for example, in the presence of data gaps.

Time window selection is accomplished using FLEXWIN, an automatic selection algorithm which identifies distinct energy arrivals using an STA/LTA ratio waveform, and isolates waveform similarities using a comprehensive set of rejection criteria. Pyatoa employs the FLEXWIN algorithm through wrapping functions of the Python port, Pyflex (Krischer, 2015b). Additional windowing criteria, such as weights based on geographic location (e.g Ruan et al., 2019), can readily be inserted into the windowing procedure via Pyatoa. In Figure 3.6, the gray STA/LTA 


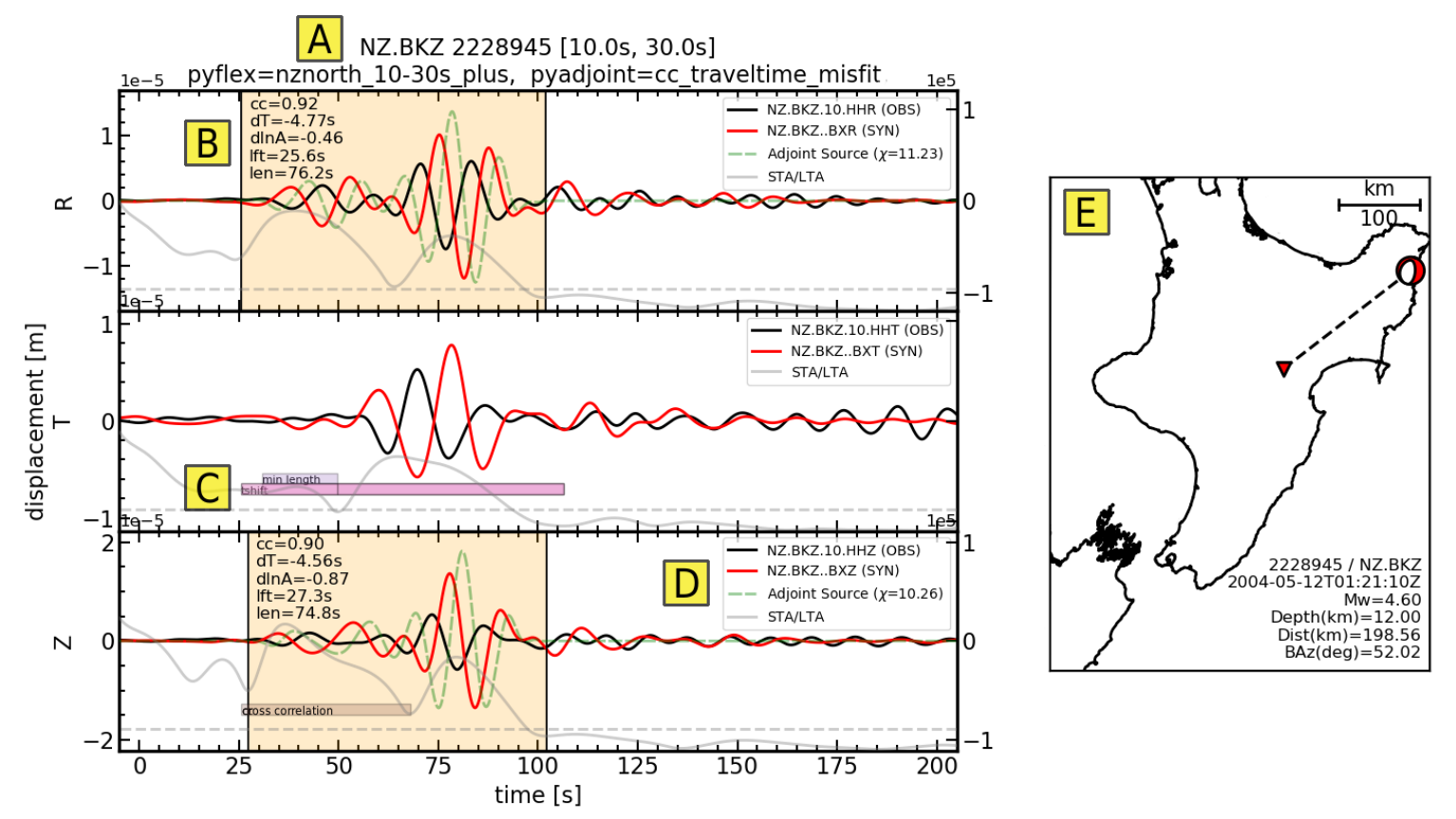

Figure 3.6: Misfit assessment for one source-receiver pair, generated using Pyatoa. Yellow labels are added for descriptive purposes. A) Waveform title, which displays relevant information like processing parameters. B) Time windows are shown with measurement information for quick assessment of waveforms and misfit. By default, cross-correlation (cc), time shift in seconds (dT), amplitude anomaly (dlnA), window start time in seconds (lft), and window length in seconds (len) are provided. C) Rejected time windows are shown as color-coded bars. Here preliminary time windows are rejected for unacceptable time shift (tshift) and minimum length (min length). D) The legend provides component identification and total calculated misfit for a single component $(\chi)$. The gray short-term-average over long-term-average waveform (STA/LTA) is used to determine preliminary windows, and is shown alongside a waterlevel (dashed grey) used for the internal rejection criteria. E) A corresponding map with useful information pertaining to the given source-receiver pair. 
waveforms and associated time windows in orange show the outputs of Pyflex. Here the peaks of the STA/LTA waveform highlight distinct arrivals in the synthetic waveform. The annotations displayed in the time windows provide useful information for misfit assessment. Time windows that were discarded due to the FLEXWIN internal rejection criteria are also shown (Figure 3.6C), giving the user a more informed understanding of waveform misfit in the absence of selected time windows.

To evaluate objective functions and generate adjoint sources, Pyatoa abstracts the functionalities of Pyadjoint (Krischer, 2015a). Within chosen misfit windows, Pyadjoint is used to evaluate the objective function and derive adjoint sources; the green dashed waveforms in Figure 3.6 are examples of adjoint sources. Pyadjoint provides a template structure that allows for the use of any objective function relating data and synthetics to be used to define misfit. Pyatoa provides an additional level of organization for Pyadjoint results to facilitate assessment of misfit in bulk. Using the functionality of Pyadjoint, adjoint sources can be stored in ASDF data sets, and/or exported into SPECFEM3D-ready file formats for use in adjoint simulations. Pyatoa provides additional utility functions to produce auxiliary files required by SPECFEM3D to launch a subsequent adjoint simulation.

\section{Assessment of inversion results}

Seismic inversions produce a large amount of data which are prohibitively timeconsuming to manually parse, organize, and understand. To help address this, Pyatoa makes use of the Python-based data analysis library Pandas (Wes McKinney, 2010; Pandas Development Team, 2020). Time windows and associated misfit values are collected and organized, at each iteration, into a useful spreadsheet-like data structure, providing an efficient tool for aggregating measurements and assessing misfit at all scales, from the single time window, to event- or station-based comparisons, to bulk analysis of each iteration. Additional automated routines allow Pyatoa to visualize waveforms, time windows, and adjoint sources (e.g. Figure 3.6), while pre-defined statistical plots summarize bulk assessment of waveform quality, window choice, and misfit characteristics. These tools are designed to inform the user on inversion behavior and to facilitate the assessment of inversion quality. 


\subsection{Synthetic inversions}

We perform realistic synthetic inversions to demonstrate the workflow, provide resolution information, and evaluate methodological choices through repeated testing. A large-scale real-data inversion has not yet been undertaken and is the subject of on-going research; however, the aim of the synthetic experiments is to lay the foundation for a real-data inversion and provide confidence that methodological choices work well for our study area.

\subsubsection{Inversion setup}

We perform a synthetic 3D inversion using a realistic numerical mesh and target perturbation checkerboard model (Figure 3.7). The checkerboard consists of $80 \mathrm{~km}$ wide, Gaussian-tapered vertical columns, with alternating peak central amplitudes of $\pm 20 \%$ perturbation. A width of $80 \mathrm{~km}$ was chosen as a reasonable size for resolvable features for the target period band of 10-30 s. The perturbation amplitude is chosen to be $20 \%$ following the results of Tape et al. (2010), who observed maximum absolute velocity changes of $30 \%$ with respect to their initial ray-based tomography model of southern California. The perturbations are chosen to be columnar in depth, since the test data (10-30 s surface waves) are mostly sensitive to lateral variations. The perturbation checkerboard is overlain on the NZ-North velocity model and used to create realistic synthetic data for 30 events gathered at 58 receiver locations (Figure 3.1).

In the iterative inversion procedure, model updates are computing using the LBFGS algorithm (Liu and Nocedal, 1989), which involves a local quadratic approximation of the objective function. We use a safeguarded backtracking line search (Modrak and Tromp, 2016) to determine the step length of model perturbation. At each iteration, $V_{p}$ and $V_{s}$ are updated, but density is not estimated due to the limited sensitivity of surface waves to density structure (Nazarian and Stokoe, 1984). Although adjoint source-parameter inversion (Kim et al., 2011) is feasible within the context of the workflow, it is not addressed, and misfit between data and synthetics is attributed to inaccuracies in the velocity models only.

Smoothing the gradient by convolution with a Gaussian is used to suppress nonuniqueness in the updated models. Based on the perturbation scales and target bandpass, 

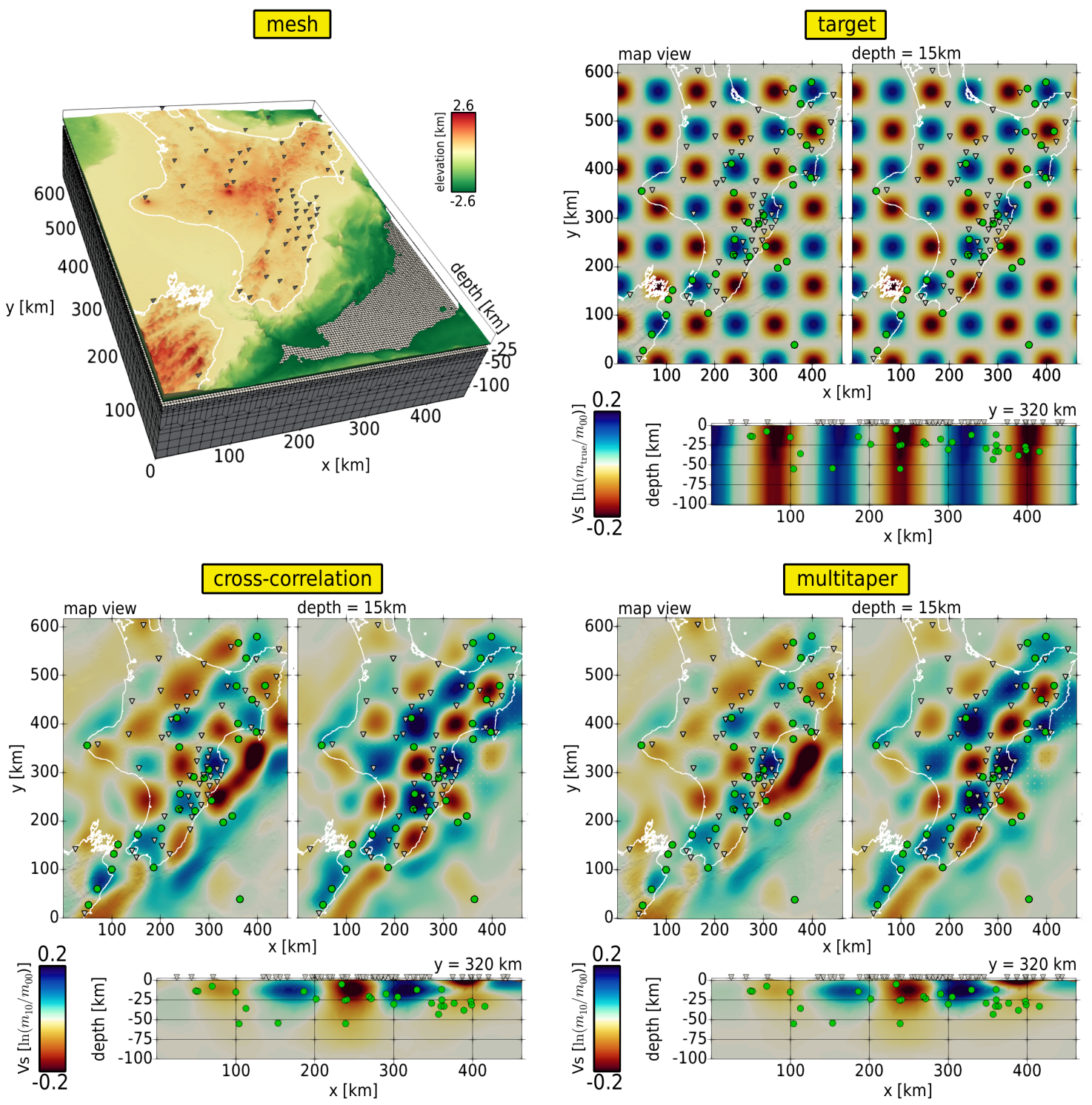

Figure 3.7: Summary figure showing setup and results of the synthetic inversions. The Cartesian numerical mesh is shown in the upper-left. In the top portion, elevation is coded by color; below, the numerical mesh is shown from depths of 20 to $100 \mathrm{~km}$. Bathymetry is purposely cut off below $-2.6 \mathrm{~km}$ to reveal the smallest elements with a size of $2 \mathrm{~km}$. Two coarsening layers can be seen at approximately 25 and $50 \mathrm{~km}$ depths, with a largest element size of $40 \mathrm{~km}$. Though not shown, the mesh continues to $400 \mathrm{~km}$ depth. The target velocity perturbations (upper-right) and recovered velocity perturbations (bottom row) are shown in map view, with a horizontal slice at $15 \mathrm{~km}$ depth, and in cross section. Cross sections are shown at a fixed Y-axis value of $320 \mathrm{~km}$, which traverses through the center of one set of $80 \mathrm{~km}$ wide checkers, where perturbations of the NZNorth velocity model are at their maximum. Colors represent net model update of $\mathrm{V}_{s}$, which saturates at the largest perturbation values of \pm 0.2 . In map view and horizontal slice, green circles show event epicentral locations. In cross section, green circles show event depths. Receiver locations are shown as inverted triangles. 
we choose horizontal and vertical half-widths of the Gaussian to be $17.5 \mathrm{~km}$ and $4 \mathrm{~km}$, respectively. Additionally, misfit windows are re-evaluated at each function evaluation, allowing the objective function to change with each iteration. Because the introduction of misfit windows is gradual, changes in the misfit function are accounted for over multiple L-BFGS updates. Finding that L-BFGS always returned a descent direction and noting that L-BFGS restarts can be quite costly (Modrak and Tromp, 2016), we refrained from restarting the optimization algorithm. To be shown, the model converges in a well-behaved manner, suggesting these are acceptable choices for this specific inversion scenario.

Updated models are analyzed based on recovery of perturbations, waveform improvement, and overall reduction in the objective function. For a given model $i$, perturbation recovery is shown relative to the starting model as the net model update $\ln \left(\mathrm{m}_{i} / \mathrm{m}_{00}\right)$, which, to first order, approximates fractional differences $\left(\mathrm{m}_{i} / \mathrm{m}_{0}-\right.$ 1). For large differences, the logarithmic misfit provides a more suitable representation of compounding changes accrued during an iterative inversion.

\subsubsection{Computational expense}

To provide a sense of computational scale for these synthetic inversions, we outline the demands entailed by our preliminary analysis. To limit the overall computational expense, inversions are stopped after 10 iterations with each simulation lasting $300 \mathrm{~s}$ seismogram length. The numerical mesh described in Section 3.2.1 is accurate to $2 \mathrm{~s}$ periods, which is over-sampled for our target period band of 10-30 s. We consequently remake the mesh with an accuracy of $10 \mathrm{~s}$, which corresponds to 124000 elements and a minimum element spacing of $2 \mathrm{~km}$ (Figure 3.7). Each simulation requires roughly 10 minutes on 80 physical cores of the New Zealand eScience Infrastructure's (NeSI) Cray XC50 supercomputer, named Māui. In the first few iterations, additional forward simulations are required by the L-BFGS algorithm to properly scale the step length, which typically results in three to five simulations per event per iteration. Once the search direction is well scaled, iterations typically require only two simulations (one forward, one adjoint) per event. An additional smoothing operation is required per iteration. Overall, a 10 iteration inversion required roughly 8000 CPU-hours, with a walltime of approximately 20 hours. After the initial manual tasks of mesh generation and parameter setup, all tasks ran fully-automated until the end of the inversion. 


\subsubsection{Resolution and parameter testing}

The synthetic inversions performed are used not only as a form of resolution testing, but also to illustrate the capability of our workflow to quantitatively guide methodological decisions for full-scale inversions. Choices of, for example, starting model, nonlinear optimization algorithm, bandpass corners, windowing criteria, smoothing lengths, and objective function, will have significant effects on the behavior, efficiency, and robustness of an inversion. Our automated workflow facilitates the use of synthetic inversions to test the effects of various input configurations on inversion behavior. Here, as an example, we show the results of inversions run using two different objective functions. One objective function is the cross-correlation traveltime misfit,

$$
\chi(\mathbf{m})=\frac{1}{2}\left[\frac{T^{o b s}-T(\mathbf{m})}{\sigma}\right]^{2},
$$

where $\mathrm{T}^{o b s}$ is the observed traveltime, $\mathrm{T}(\mathbf{m})$ the corresponding synthetic traveltime for a model $\mathbf{m}$, and $\sigma$ an optional weighting of the measurement uncertainty (Tromp et al., 2005). The other objective function is the multitaper misfit,

$$
\chi(\mathbf{m})=\frac{1}{2} \int_{0}^{W} W_{P}(\omega)\left|\frac{\tau^{d}(\omega)-\tau^{s}(\omega, \mathbf{m})}{\sigma_{P}(\omega)}\right|^{2} d w
$$

where $\tau^{d}(\omega)$ is an observed frequency-dependent phase measurement, $\tau^{s}(\omega, \mathbf{m})$ is the synthetic phase measurement for model $\mathbf{m}, \sigma_{P}$ is a measurement uncertainty, and $W_{P}$ is a tapered frequency-domain window (Tape, 2009). Both objective functions are defined for a single time window. Although we only show results from two very-similar synthetic inversions, we emphasize that this capability can be extended to compare the effects of any range of input parameters.

\section{Recovered perturbations}

We qualitatively analyze perturbation recovery for the two inversions. Figure 3.7 shows the target $V_{s}$ perturbations compared with final recovered perturbations using the cross-correlation traveltime misfit and multitaper misfit in map view, a horizontal slice at $15 \mathrm{~km}$, and in cross section. Both synthetic inversions are able to recover amplitudes and spatial distribution of perturbations for areas with sufficient data coverage. Visually, the two objective functions exhibit near-identical model recovery. As expected, areas with no source-receiver coverage show little 
to no change from the starting model. Features are resolved at the surface with pronounced smearing due to the uneven source-receiver distribution and the use of primarily surface-wave derived measurements. Features are more sharply resolved at $15 \mathrm{~km}$ depth, which we attribute to enhanced coverage from events, which have an average depth of roughly $25 \mathrm{~km}$. Cross sections across a single row of checkers show that $\mathrm{V}_{s}$ perturbations are recovered adequately to $25 \mathrm{~km}$ depth, and best below land. Recovery in the oceanic forearc region is constrained mostly to the upper $10 \mathrm{~km}$. As expected, resolution is worse on the western half of the North Island where source and receiver coverage is more sparse. Recovered perturbations suggest that the source-receiver distribution in the chosen domain is capable of recovering features on land and immediately offshore, with depth coverage mostly constrained to the upper $25 \mathrm{kms}$.

\section{Misfit reduction}

Convergence behavior for each model is shown in Figure 3.8. For both objective functions, after 10 iterations, the total misfit reduces by roughly a factor of 3 with respect to the initial model. The cross-correlation inversion shows negligibly better misfit reduction compared to multitaper. Figure 3.8 also shows the total number of measurements made for each model. Approximately 5000 measurements would be expected if one time window was chosen for each of the three components of every source-receiver pair. Roughly 7000 initial measurements are made for the initial model, which increases by approximately 700 measurements at model 10 . Both objective functions see a similar increase in total number of measurements over the course of the inversion. For this specific synthetic inversion, the convergence behavior of multitaper and cross-correlation misfits is almost identical. This is unsurprising, however, for two phase-based objective functions, evaluated for a long-period bandpass over a limited number of measurements.

Two additional, identical inversions were performed, where a fixed set of windows, chosen for the initial model, were re-used throughout the entire inversion. With respect to the case shown above, the resultant convergence plot and mapviews of recovered perturbations show similar comparative behavior between the two objective functions (Figure D.5). Although misfit reduction is more constrained and surface features less sharply resolved, this can primarily be explained by the restricted number of measurements. We emphasize, however, that the results 


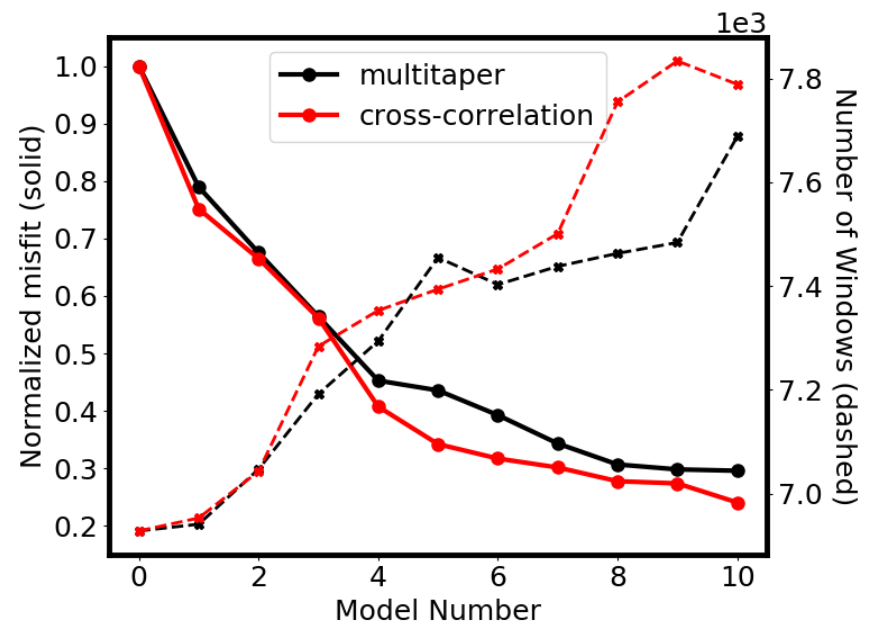

Figure 3.8: Synthetic inversion convergence behavior comparing the traveltime crosscorrelation misfit and multitaper misfit objective functions. Misfit normalized to start at 1. Comparative behavior is almost identical for this specific synthetic test case, unsurprising for a comparison of two phase-based objective functions. New misfit windows are evaluated at each function evaluation, showing an on-average increase in measurement number throughout the inversion. This is interpreted as a sign of increasing waveform improvement for each model update.

shown here are unique to our specific synthetic-inversion scenario, and that the effect of objective function, windowing parameters, etc., on inversion behavior remains contingent on initial conditions.

\section{Waveform improvement}

Misfit histograms are used to document overall measurement improvement after 10 iterations, from the initial (M00) to the final model (M10). Figure 3.9 shows results for the cross-correlation traveltime misfit inversion only, as the multitaper inversion results are very similar. Average time shift (Figure $3.9 \mathrm{~A}$ ) is reduced from $-0.7 \pm 2.1 \mathrm{~s}$ to $0.0 \pm 1.0 \mathrm{~s}$. Amplitude differences are also reduced in mean and standard deviation; amplitude information is not considered in the phase-based objective function, so we would not expect amplitude differences to reduce to zero. Peak cross correlations are seen to shift towards a peak value of 1.0, and relative start times of measurements increase mostly in measurements made after $100 \mathrm{~s}$, suggesting that an increased number of later arrivals, and/or large propagation distances, are chosen using the final model.

A representative example of waveform improvement over the course of the inver- 

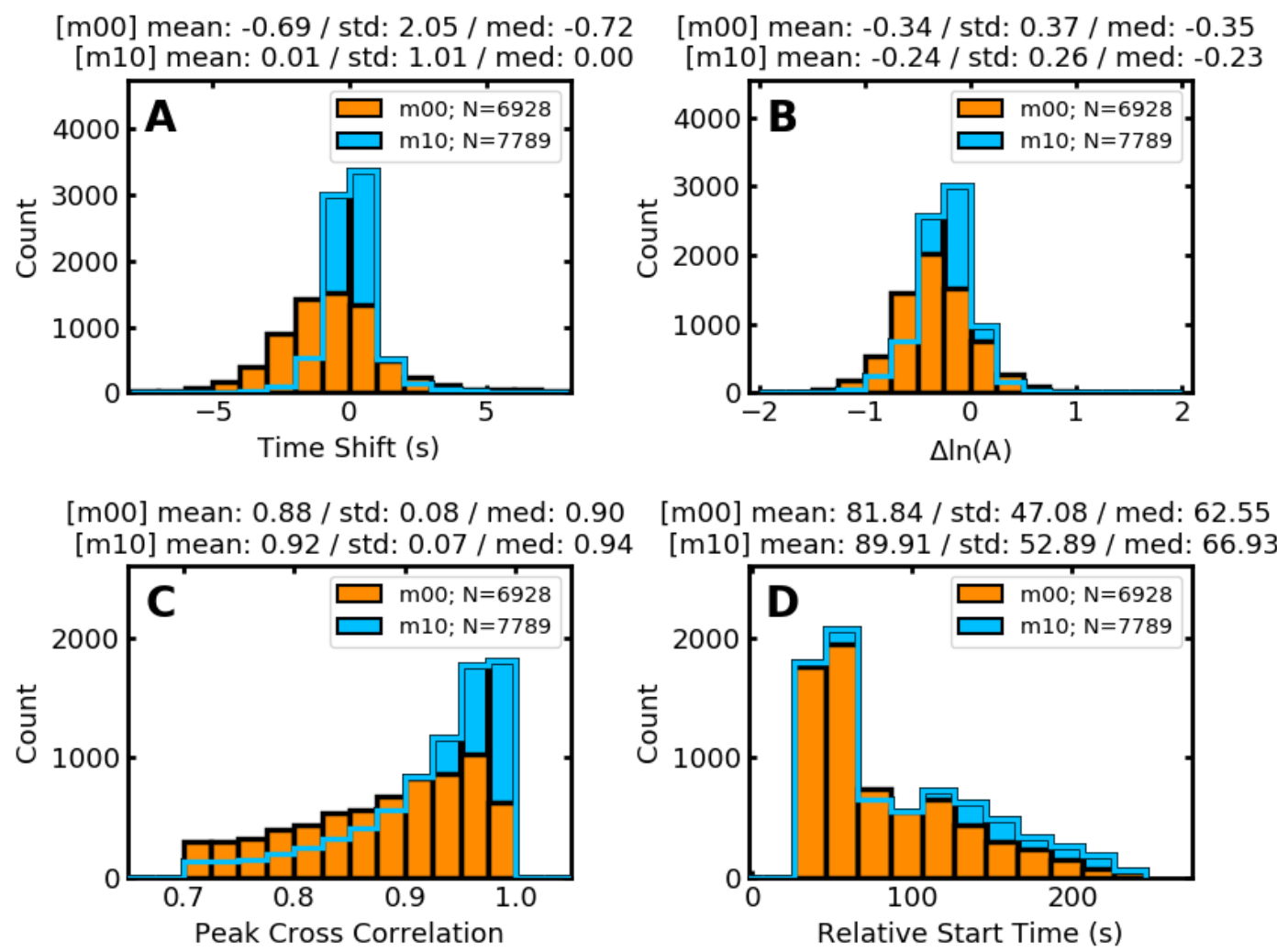

[m00] mean: $81.84 /$ std: $47.08 /$ med: 62.55

[m10] mean: $89.91 /$ std: $52.89 /$ med: 66.93

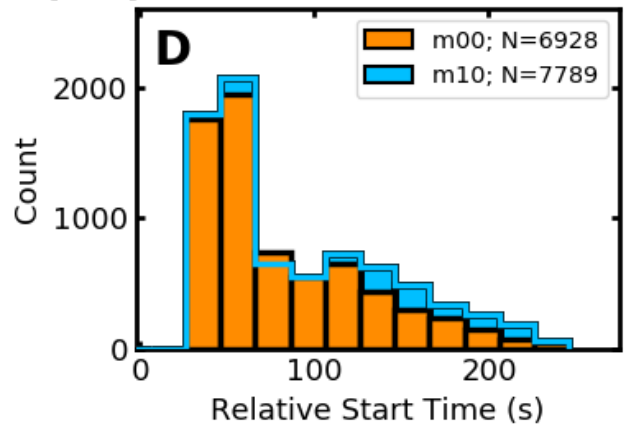

Figure 3.9: Misfit histograms for a synthetic inversion using the cross-correlation traveltime misfit function. Comparisons are made between the initial model M00 (orange) and final model M10 (blue). A) Cross correlation time shift in seconds. B) Amplitude ratio dlnA (Equation 3.1). C) Peak cross correlation value. D) Window start time relative to event origin time. Mean, standard deviation, and median for each quantity and model are provided in the title. The number of measurements for each model is given in the respective legends. Histograms illustrate an overall improvement in waveforms. Results are similar for the multitaper inversion. 

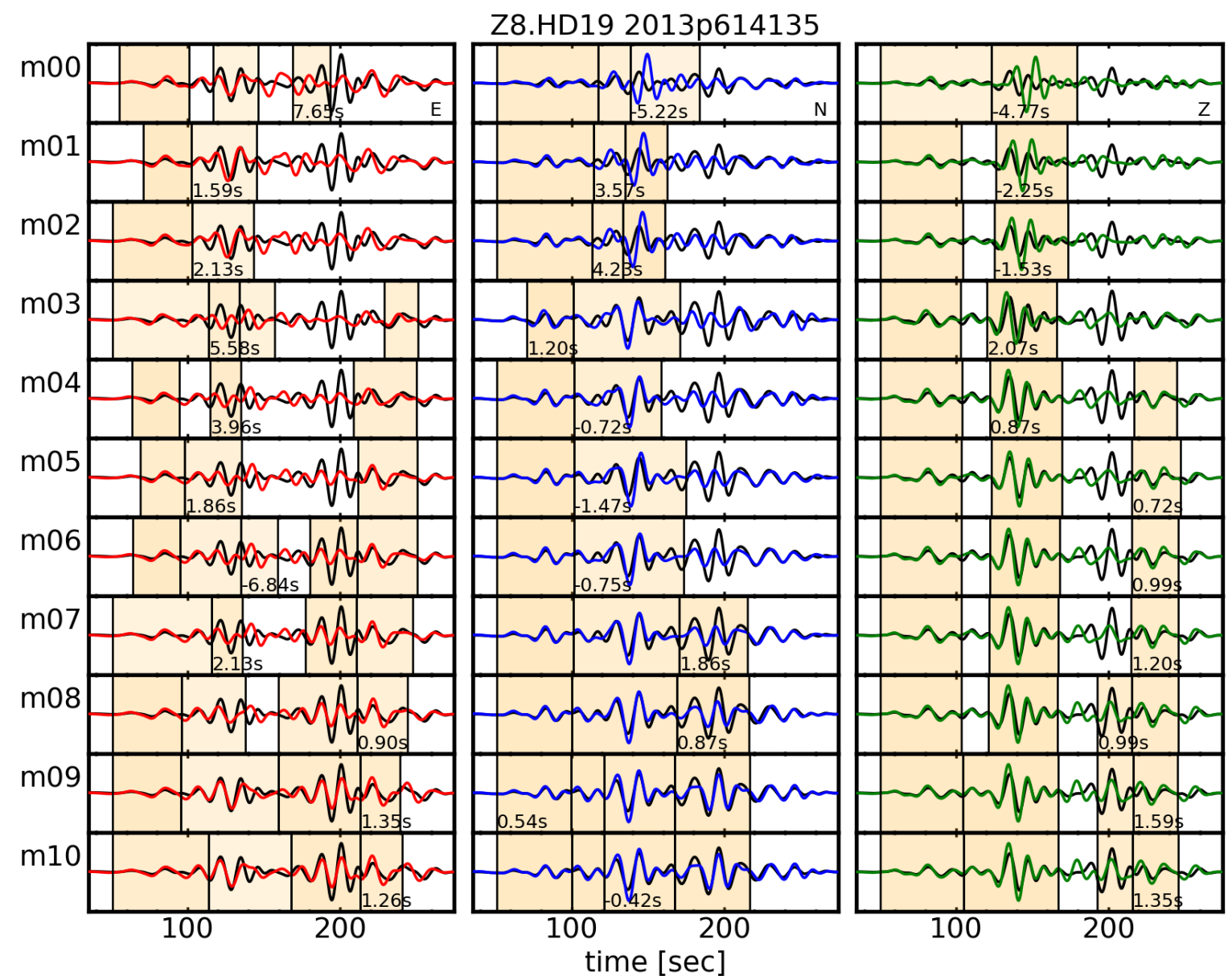

Figure 3.10: An example of synthetic inversion waveform improvement for the crosscorrelation traveltime misfit function. Waveforms are shown from the initial model (M00) to the final model (M10) for three components: east-west (E), north-south (N), and vertical $(Z)$. Observation waveforms shown in black, synthetic waveforms shown in color. Waveforms shown in units of displacement. Time windows chosen by Pyflex are shown as orange windows. For simplicity, only the maximum time shift for each pair of waveforms is shown. Time windows are re-evaluated for each new model.

sion is shown for three components of a source-receiver pair in Figure 3.10. Observed and synthetic waveforms, along with misfit windows and maximum time shift values are shown for each progressive model update. Large initial waveform differences, characterized by large time shifts and amplitude ratios, are improved significantly throughout the inversion. By model 10, almost all available sections of the waveforms are windowed and aligned, and maximum time shifts are small, or found in the latest arrivals ( $>200 \mathrm{~s}$ ). 


\subsection{Discussion}

\subsubsection{Choice of workflow tools}

Our choice of workflow tools is based on the requirements of our earthquake tomography problem. We use existing tools like SPECFEM3D, because they are accurate, efficient, and available. SeisFlows has been chosen as the optimization tool in preference to other contenders such as SEISCOPE (Métivier and Brossier, 2016), because it is written in Python and provides built-in integration with SPECFEM2D, 3D Cartesian, and 3D Globe. For misfit quantification and workflow management, we considered LASIF (Krischer et al., 2015a), which provides a framework for large-scale inversions, however attractive capabilities such as automated launching of simulations on HPC systems, or an accompanying optimization library for model updates, are not featured. The Salvus software suite (Afanasiev et al. 2019) provides an all-encompassing approach to full-waveform inversion but contains components that are currently closed-source. Similarly, inversion frameworks used in various published studies (e.g. Chen et al., 2007b; Lee et al., 2014c) are not currently available. To meet the requirements of the earthquake tomography problem, we have opted to design our own toolbox. We envision the flexible nature of SeisFlows, enhanced with the detailed measurement capabilities of Pyatoa, forming a toolchain applicable to future earthquake tomography problems.

\subsubsection{Potential applications of an automated inversion workflow}

The methodological framework developed here is general and should be applicable, with minor modifications, to other regions with suitable data coverage and candidate velocity models. The comparison of synthetic inversions in this study is a simple example to illustrate the powerful capacity of an automated inversion workflow to inform and assess any choice of input parameters for a seismic inversion. The synthetic checkerboard inversion is a useful method for quantifying the resolving power of the source-receiver distribution for a given region, although it should not be seen as a replacement for rigorous quantitative analyses of model resolution, such as the point spread function of Fichtner and Trampert (2011b). Though not implemented, the capability for adjoint moment tensor inversions (Kim et al. 2011) is feasible within the context of this workflow, and it remains 
a potential avenue for future efforts towards joint velocity model and source parameter inversions.

As with the 2D synthetic comparisons of optimization schema by Modrak and Tromp (2016), the ability to easily repeat inversions with slight modifications in input parameters opens up a wide range of possibilities for assessing inversion behavior. For example, with the availability of various objective functions for seismic inversions, such as instantaneous phase differences and envelope ratios (Bozdağ et al., 2011), or amplitudes, attenuation, and differential traveltimes (Tromp et al. 2005), more detailed analyses can be performed to understand the impact of objective functions on tomographic inversions.

\subsubsection{Towards a full-scale North Island inversion}

One overarching goal of this work is to set the foundation for a full-scale seismic inversion. Given our analysis of the NZ-North velocity model, as well as the results of our synthetic inversions, we expect a full-scale inversion to result in a substantial overall reduction in full-waveform misfit between data and synthetics, and improved accuracy in crustal images for the North Island. Our efforts will focus towards better resolution of shallow crustal structure, and deeper understanding of the Hikurangi subduction zone, Taupō Volcanic zone, and North Island tectonics. If $2 \mathrm{~s}$ waveforms can be fit, then we should expect waveform sensitivity in the shallow region of approximately $1-2 \mathrm{~km}$, corresponding to the lowest wavespeeds $\left(\sim 1 \mathrm{~km} \mathrm{~s}^{-1}\right)$ in our starting model. Interpretations will be directly compared with results of active seismic experiments, geologic profiles, and tectonic studies of the area.

Our full-scale inversion will begin by fitting large scale features corresponding to long-period waveforms, subsequently moving to shorter periods with a target period band of 2-30 s. Data are available for 250 earthquakes recorded on up to 100 broadband seismic stations, including temporary seismic networks throughout the North Island (Figure 3.11). Smoothing at large spatial scales may be necessary in early iterations to promote resolution of large scale structures before attempting to image finer details. If necessary, empirical preconditioners can be used to down weight the contribution of near-source and near-receiver structure to model updates, as in Fichtner et al. (2009). Additionally, data-category and geographical 
weighting of measurements can assist with the uneven source-receiver distribution in our domain (Ruan et al., 2019). A subset of our event catalog will not be included in the inversion, and retained for post-hoc analysis of the inversion's ability to resolve true Earth structure. Full-scale resolution testing, using a point spread function, Hessian kernels, and/or full-scale checkerboard inversion including vertical variations, can be performed to quantify the resolving capabilities of the full source-receiver distribution. Forward simulations on the numerical mesh described in Section 3.2.1 require 25 minutes on 160 physical cores of Māui. Basing our estimates on the synthetic inversions of Section 3.4.2, with a conservative expectation of 20 iterations to reach convergence, we estimate a full-scale inversion using 200 events to require on the order of 1000000 CPU-hours.

\subsection{Conclusions}

We present an automated inversion workflow for earthquake-based tomography using adjoint methods. The workflow is constructed from both newly-developed and existing Python tools built around the widely used numerical solver SPECFEM3D Cartesian, providing end-to-end capability for adjoint tomography. We verify the workflow through automatic recovery of 3D checkerboard perturbations via iterative model updates on an HPC system. We also use these synthetic inversion results to carry out resolution analysis and illustrate parameter testing capabilities. Additionally, synthetic seismograms generated using a candidate 3D velocity model of New Zealand show reasonable misfit with respect to observed waveforms, illustrating the potential for improvement of the velocity model using adjoint methods. Comparisons with a 1D North Island velocity model and similarly sized study regions provide optimism that NZ-North provides a strong candidate starting velocity model for adjoint tomography. The success of the synthetic inversions and the reasonable misfit of the candidate velocity model, provide confidence moving toward a large-scale seismic inversion of the North Island, New Zealand. Synthetic resolution tests highlight the potential for substantial model improvement both onshore and offshore, with well-resolved velocity changes extending from the surface to roughly $25 \mathrm{~km}$ depth. The automated and modular nature of the workflow means that a wide range of input parameters may be tested and chosen, to efficiently guide convergence of the inversion. A full-scale high-resolution seismic inversion of the North Island, New Zealand, using spec- 


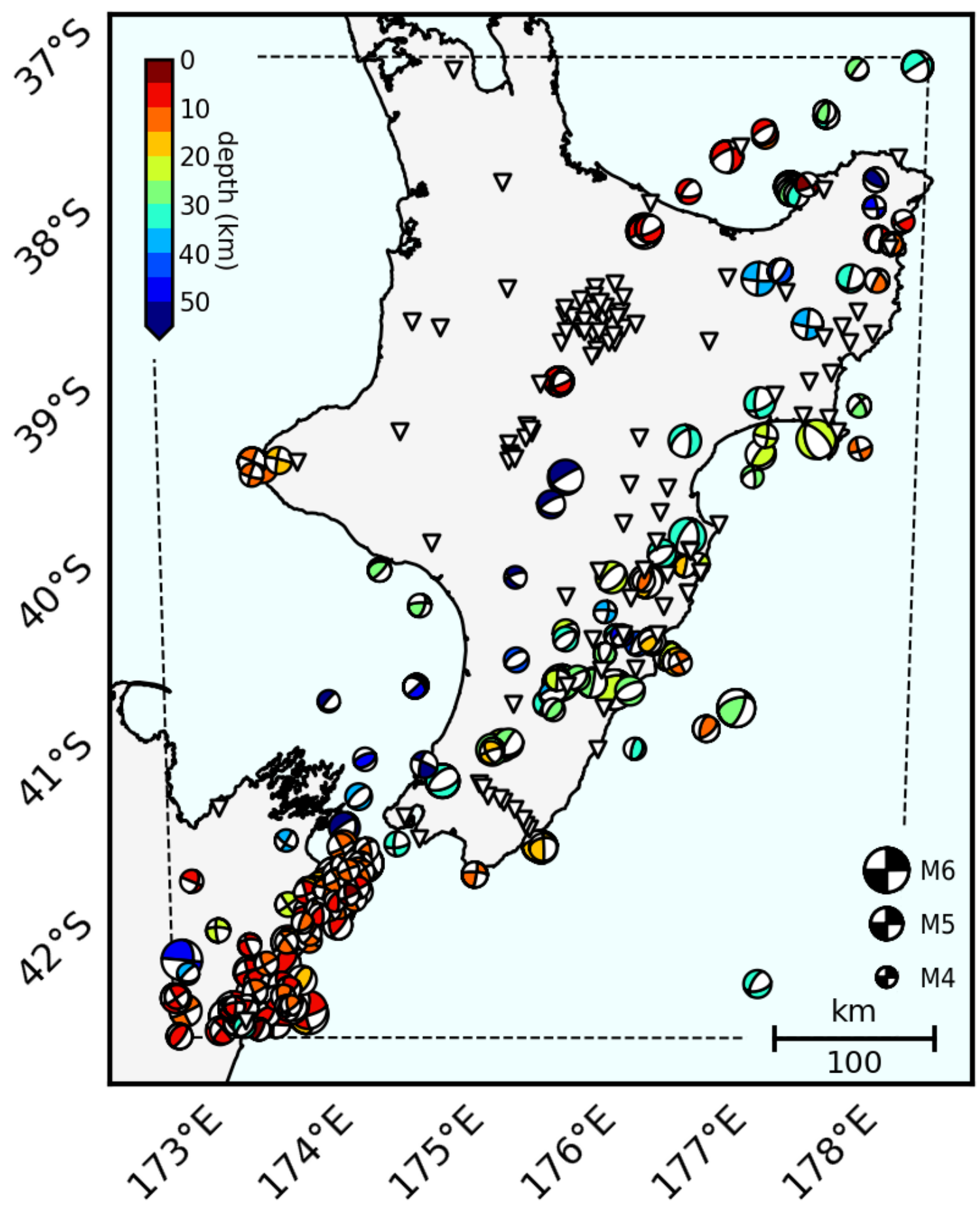

Figure 3.11: Potential setup for a full-scale inversion within the NZ-North domain (dashed line). 250 earthquakes with magnitudes $4.4 \leq \mathrm{M}_{\mathrm{w}}<6.0$ are shown as moment tensors, scaled by size with color coded by depth. 100 broadband seismic station locations, consisting of New Zealand's permanent seismic network and temporary networks throughout the North Island, are shown as inverted triangles. The simulation domain used in this study is shown as a dashed black line. 
tral element and adjoint methods, is the subject of on-going research. 


\section{Chapter 4}

\section{Adjoint tomography of the North Island of New Zealand ${ }^{[}$}

We undertake the first application of adjoint tomography in New Zealand to improve an existing 3D velocity model containing the Hikurangi subduction zone and the North Island of New Zealand. The chosen study area offers a unique opportunity for imaging material properties near an active subduction zone, due to the availability of well-recorded earthquakes in close proximity to the plate interface. We perform an iterative inversion using spectral element and adjoint methods. Data include up to 1800 unique source-receiver pairs used to fit waveforms with periods 4-30 s, relating to minimum waveform sensitivity on the order of 5$10 \mathrm{~km}$. In total, 28 model updates of $\mathrm{V}_{p}$ and $\mathrm{V}_{s}$ are undertaken with an L-BFGS nonlinear optimization algorithm, totalling an approximate computational cost of 500000 CPU-hours. Overall time shift between observed and synthetic seismograms is reduced from an initial mean and standard deviation of $2.0 \pm 3.9 \mathrm{~s}$ to $0.5 \pm 3.7 \mathrm{~s}$, while $\mathrm{V}_{p}$ and $\mathrm{V}_{s}$ show up to $\pm 30 \%$ change relative to initial values. Resolution analyses suggest that velocity changes are most strongly resolved on-land

\footnotetext{
${ }^{1}$ As of August 2021, two companion manuscripts based on results presented in this chapter are under peer review with the Journal of Geophysical Research: Solid Earth: (1) Bryant Chow, Yoshihiro Kaneko, Carl Tape, Ryan Modrak, Nick Mortimer, Stephen Bannister, John Townend, Strong upper-plate heterogeneity at the Hikurangi subduction margin (North Island, New Zealand) imaged by adjoint tomography, (2) Bryant Chow, Yoshihiro Kaneko, John Townend, Evidence for deeply-subducted lower-plate seamounts at the Hikurangi subduction margin: implications for seismic and aseismic behavior
} 
and directly offshore, at depths above $30 \mathrm{~km}$, with at least $1 \%$ velocity changes seen down to $100 \mathrm{~km}$ depth.

\subsection{Introduction}

Seismic tomography is an exceedingly powerful tool for illuminating the complexity of Earth structure, from crust to core. In New Zealand, for example, ray-based tomographic images have been used to characterize the complex Hikurangi subduction zone and its associated tectonic processes (Eberhart-Phillips and Reyners. 2012; Eberhart-Phillips et al., 2015, 2017). Recent progress in the field of tomography now allows for more powerful full-waveform imaging techniques, which take advantage of modern advances in computational power to leverage large sections of the seismic waveform. Earthquake-based full-waveform tomography using spectral element and adjoint methods has been shown to improve synthetic waveforms generated using ray-based tomography models, and consequently image previously unseen velocity features (e.g. Fichtner et al., 2009; Tape et al., 2010; Zhu et al., 2015, Bozdağ et al., 2016). Despite its increased resolving power, the computational complexity of adjoint tomography, and more stringent data requirements, limit its applicability to domains with sufficient seismicity and seismic network coverage.

In Chapter 3 we demonstrated the feasibility of applying full-waveform tomography to the North Island of New Zealand, using spectral element and adjoint methods. Building upon this work, we perform adjoint tomography for the New Zealand region to improve an existing 3D velocity model, and present the results of the tomographic inversion. Our new velocity model will be defined in terms of seismic velocities $\left(\mathrm{V}_{p}, \mathrm{~V}_{s}\right)$, and their ratio $\left(\mathrm{V}_{p} / \mathrm{V}_{s}\right)$. The three main goals of this chapter are to:

1. Use the automated workflow tool developed in Chapter 3 to perform adjoint tomography for the North Island of New Zealand;

2. Present and assess an updated high-resolution velocity model generated using 4-30 s seismic waveforms;

3. Quantify the resolution of the updated velocity model and identify the most

\footnotetext{
${ }^{2}$ also published in Chow et al. (2020)
} 
striking relative velocity changes.

The chapter begins with a description of the data used in the inversion (Section 4.2), followed by an explanation of methodologies (Section 4.3). Section 4.4 presents the final velocity model M28, and accompanying resolution analysis (Section 4.5). We save interpretations and implications of velocity changes for the following chapter (Chapter 5).

\subsection{Data}

The underlying goal of adjoint tomography is to fit synthetic seismograms, generated using a starting velocity model, to earthquake observations recorded by seismic stations. The data required to perform such an inversion include observed seismic waveforms, moment tensors describing the earthquakes, and a velocity model in which to simulate seismic wave propagation. In the following section we discuss the data used in this seismic inversion. Some information may be repeated from previous chapters for clarity.

\subsubsection{Study area and starting model}

The study area in this chapter is the same as "NZ-North" defined in Section 3.3.2. In this section we provide additional information detailing the choice of domain bounds. "NZ-North" defines a region consisting of the central and southern North Island, and the northern South Island (Figure 4.1), with edges chosen primarily based on source and receiver locations, as well as concerns for computational expense. Limited station coverage, and a lack of usable events ruled out the northern regions of the North Island, north of $37^{\circ} \mathrm{S}$. The eastern boundary $\left(178.5^{\circ} \mathrm{E}\right)$ is limited to the sub-aerial extent of the North Island, chosen to minimize the amount of deep-ocean model space containing limited to negligible data coverage. The southern $\left(42.5^{\circ} \mathrm{S}\right)$ and western $\left(173^{\circ} \mathrm{E}\right)$ boundaries were chosen to include a number of aftershocks and related seismicity from the $2016 \mathrm{M}_{\mathrm{w}} 7.8$ Kaikōura earthquake. In this study, all coordinates were converted to the UTM 60S coordinate system, defining a rectangular grid covering an approximate size of $450 \mathrm{~km}$ in the $X$-direction (approximately east-west) by $600 \mathrm{~km}$ in the Y-direction (approximately north-south), by $400 \mathrm{~km}$ in the Z-direction (positive $\mathrm{Z}$ up). 
The initial velocity model used in this work is defined by the NZ-Wide2.2 velocity model of (Eberhart-Phillips et al., 2020b), developed using ray-based traveltime tomography, and improved in areas with joint inversions of Rayleigh-wave group velocity maps (Eberhart-Phillips and Fry, 2017), and joint inversions with teleseismic surface waves (Eberhart-Phillips and Fry, 2018). This 3D velocity model defines $\mathrm{V}_{p}, \mathrm{~V}_{p} / \mathrm{V}_{s}$, density and attenuation (Eberhart-Phillips et al., 2015, 2017, 2020a) for the entire New Zealand region. In this work we derive a corresponding $\mathrm{V}_{s}$ model using the NZ-Wide2.2 $\mathrm{V}_{p}$ and $\mathrm{V}_{p} / \mathrm{V}_{s}$ models. Due to the eastern edge of the domain extending further than the available domain of the starting model, a roughly triangular shaped region in the south-eastern offshore corner of the starting model was extrapolated from edge values to create a continuous starting model. In Chapter 3, we assessed waveform misfits for 250 earthquakes using this starting velocity model, and show that data-synthetic time shifts for $>25000$ measurements has a mean and standard deviation of $2.0 \pm 3.7 \mathrm{~s}$, concluding that this is a feasible candidate velocity model for adjoint tomography.

\subsubsection{Earthquake sources}

From the 250 earthquakes initially gathered in Section 3.3.2 (Figure 3.11), we select 60 earthquakes with high signal-to-noise ratio for use in our inversion (Figure 4.1). Table F.2 provides detailed source information for the events used in this inversion. Surface waves are the dominant signals for the chosen period band of waveform data (4-30 s). As previously mentioned, we choose events with magnitudes ranging $4.5 \leq \mathrm{M}_{\mathrm{w}}<6.0$, and depths above $60 \mathrm{~km}$. Waveforms from events with magnitudes $\mathrm{M}_{\mathrm{w}}<4.5$ are recorded by only several stations in the network, limiting their usefulness at the regional scale. Events with $\mathrm{M}_{\mathrm{w}} \geq 6.0$ are ruled out because our simulations represent earthquakes as point sources, and large magnitude events often require finite-fault solutions or unique source time functions for accurate synthetic waveforms. Moment tensors in New Zealand are routinely calculated by GeoNet (Ristau, 2008) and historically available for regional earthquakes since 2003.

A large number of events in the initial catalog consisted of foreshock and aftershock sequences, which densely clustered in certain regions of the domain with spatially and mechanistically similar earthquakes that produced near-identical waveforms at the period range of interest (4-30 s). Without adequate considerations 
for weighting of these events (e.g. Ruan et al. 2019), repeated contributions from these source-receiver paths were observed to have an undesired effect on the inversion. Stacked contributions from these paths would mask out more unique source-receiver paths during the inversion, leading to anomalously strong contributions in regions with dense event clustering. To down weight the contributions of such events, we perform event declustering, where events are grouped and selected based on hypocenter and magnitude, providing more uniform coverage within the domain by removing very similar events.

During the declustering procedure, we impose an additional constraint that events recorded on any temporary stations be prioritized, to preferentially include data from unique receiver locations. The final number of declustered events from the initial catalog was selected manually. We choose a final catalog size of 60 events to maximize the number of unique event locations without including too many similar locations or moment tensors (Figure 4.1). An additional 60 events ranging in time from 2005 to 2021 are set aside as a validation catalog for later post-hoc model assessment (Section 4.4.3; Table F.3).

\subsubsection{Receivers}

Broadband seismic data is collected for 88 three-component broadband stations, consisting of permanent and temporary networks in New Zealand (Figure 4.1). The permanent seismic network of New Zealand (https://www.geonet.org.nz/) is operated by GeoNet, with 38 broadband stations located within the domain. An additional 50 temporary network broadband stations are included where applicable.

In addition to the permanent network of New Zealand, temporary seismometer deployments throughout the North Island are used to enhance coverage of the permanent network. In the southern Hawke's Bay region, the Broadband East Coast Network (BEACON) was a temporary deployment of 20, broadband, threecomponent, Guralp CMG-40T stations which recorded continuously between 2017 and 2019 (Section 2.3). BEACON was deployed specifically to provide additional coverage over the locked-to-creeping transition zone on the plate interface. In the southern North Island, the Seismic Array Hikurangi Experiment (SAHKE) transect consisted of a line of short-period seismic receivers deployed perpendicular 

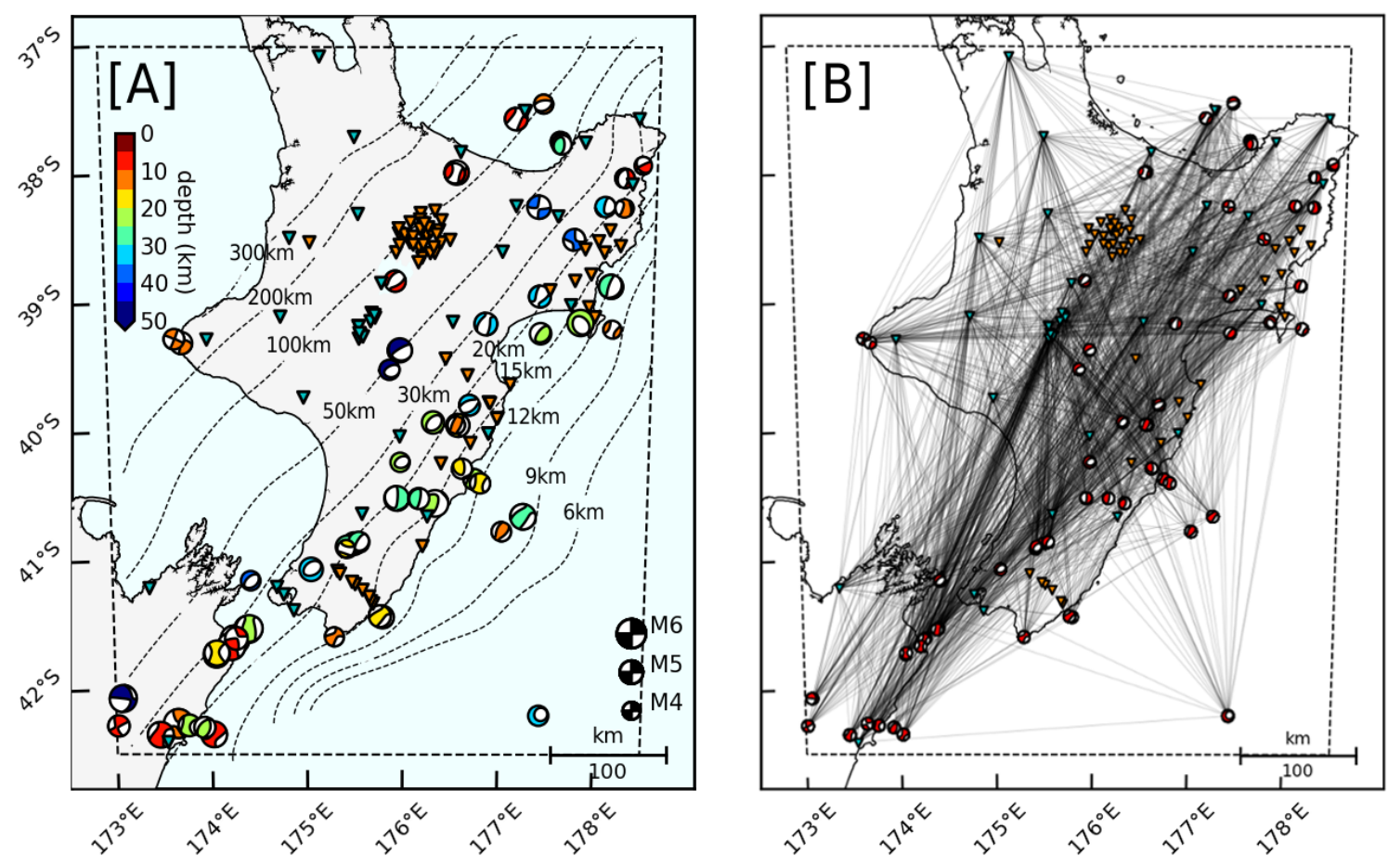

Figure 4.1: Sources and receivers included in the inversion. a) 60 earthquakes shown as focal mechanisms, color coded by depth and scaled by magnitude. 88 broadband seismic stations shown as inverted triangles, with 38 permanent network (GeoNet) stations colored blue, and 50 temporary network stations colored orange. Plate interface model of Williams et al. (2013) shown as labelled, dashed lines. b) Source-receiver ray paths for the first iteration of the inversion. Sources and receivers same as in (A). Connecting raypaths only shown for sources and receivers that have at least one measurement window. Darker patches represent areas with high ray-concentration. 
to the trench to capture offshore shots (Henrys et al., 2013) and image plate interface characteristics. A number of these sensors were colocated with broadband receivers, which are included in our dataset. Two additional deployments of seismometers, one focused on the Taupō Volcanic Zone, the other on the Gisborne region in the eastern North Island, provide additional coverage. In total, 1800 unique source-receiver paths are used (Figure $4.1 \mathrm{~B}$ ), with temporary network data providing roughly $8 \%$ of the initial dataset. Tables F.4 and F.5 provides additional details on the receivers used in this inversion.

\subsection{Methods}

We undertake adjoint tomography following the methodologies and automated workflow tools outlined in Chapter 3 . In this section we repeat some of that information to detail the specific methodological choices made during this inversion.

\subsubsection{Forward problem, meshing, misfit quantification}

The forward problem solves the seismic wave equation to generate synthetic seismograms. Numerical wave propagation simulations are performed using the opensource spectral element solver SPECFEM3D Cartesian (Komatitsch and Tromp. 2002a b). Waveform processing and misfit quantification are accomplished using the open-source package Pyatoa (Section 3.3.2), and workflow management and nonlinear optimization are addressed with the open-source package SeisFlows (Modrak et al., 2018). Simulations are performed on the New Zealand eScience Infrastructure's high performance computer, named Māui. The underlying numerical meshes are created using SPECFEM3D's internal meshing software, Meshfem3D. Our numerical domain occupies a cuboid shape with side lengths roughly 450 and $600 \mathrm{~km}$. At depth, two interfaces coarsen the mesh at approximately 10 and $50 \mathrm{~km}$, because increasing velocities with depth reduce the minimum required element size (Section 2.1.3). At each interface, horizontal element spacing doubles and vertical element spacing triples. The mesh bottom is defined at $400 \mathrm{~km}$ to avoid numerical reflections from the bottom boundary. Topography and bathymetry are included, and interpolated to $1 \mathrm{~km}$ spacing from SRTM-30P (Becker et al., 2009). Elevations at the top of the mesh range $\pm 3 \mathrm{~km}$, and no water layer is included. 

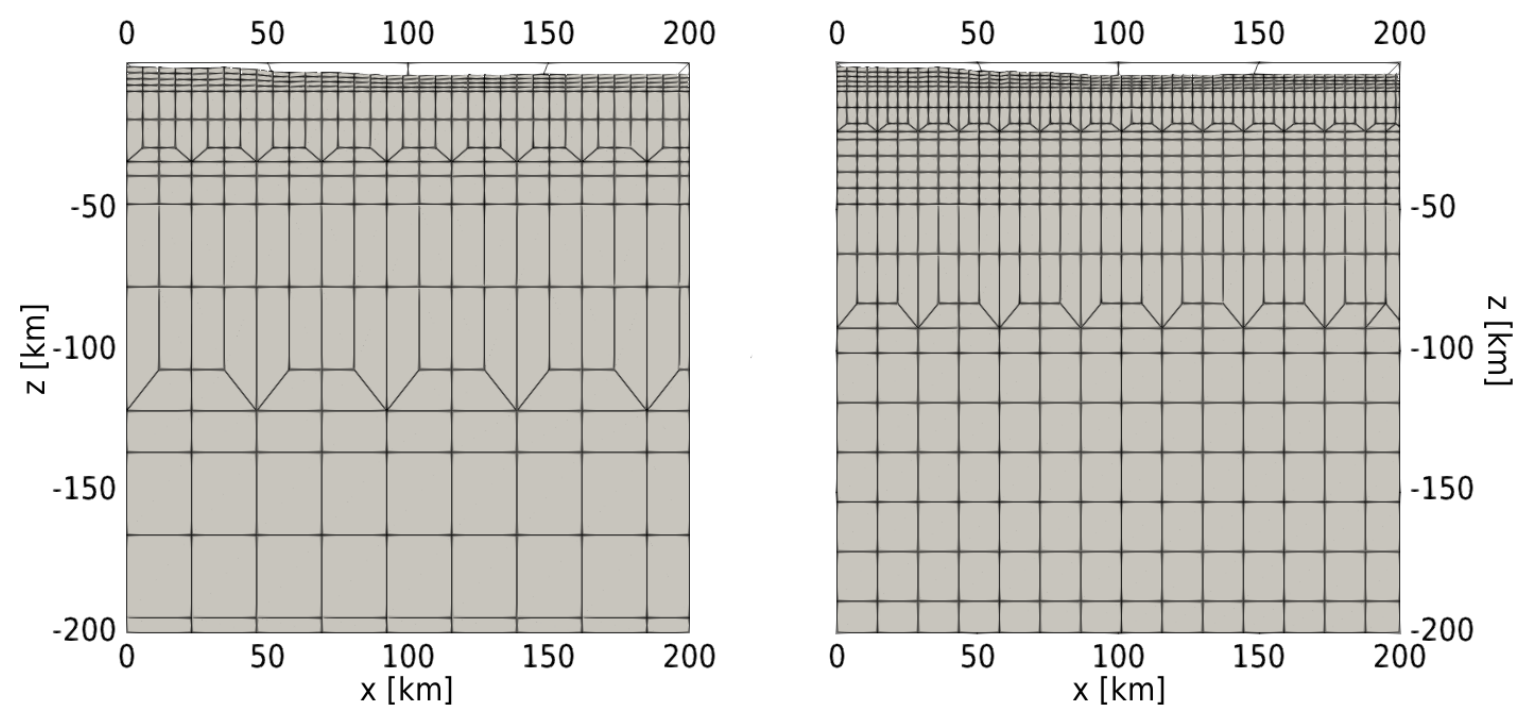

Figure 4.2: A comparison of mesh resolutions used during this inversion. (Left) Sideon view of of the coarse resolution mesh accurate to $8 \mathrm{~s}$ period with minimum element spacing of $2 \mathrm{~km}$. (Right) Side-on view of the fine resolution mesh, accurate to $2.5 \mathrm{~s}$ period with minimum element spacing of $1 \mathrm{~km}$.

The computational expense of an inversion is directly dependent on the minimum resolved period. Consequently we use different mesh resolutions at different stages of our inversion to decrease computational expense. A coarse mesh with minimum element spacing $2 \mathrm{~km}$, accurate to $8 \mathrm{~s}$ period, is used for initial longperiod waveforms (Figure 4.2). When the minimum waveform period fell below $8 \mathrm{~s}$, a similar, but finer resolution mesh with minimum element spacing of $1 \mathrm{~km}$, accurate to $2.5 \mathrm{~s}$ period, was substituted (Figure 4.2). The coarse and fine resolution meshes contained 88000 and 220000 elements, respectively, and all simulations were run for $300 \mathrm{~s}$ seismogram length. Details regarding the computational expense are provided in Section 4.4.4.

For misfit quantification, we follow the automated inversion workflow laid out in Section 3.3. To selectively restrict data included in the inversion, an automatic time-windowing algorithm is applied (Maggi et al., 2009). This procedure selects time windows in which data and synthetics are similar (i.e. high cross-correlation, low amplitude ratio), and ignores undesirable signals such as low signal-to-noise ratio observations. User-defined windowing parameters dictate the quality of input data (Table A.1). Multiple windows can be chosen for each component, and window lengths are dependent on waveform bandpass, with shorter windows used on higher-frequency waveforms to select small-wavelength features. Within 
a given time window $i$, data-synthetic misfit is calculated based on the crosscorrelation traveltime misfit function. This is provided in Equation 3.2, but we repeat it here for clarity:

$$
\chi_{i}(\mathbf{m})=\frac{1}{2}\left[\frac{T_{i}^{o b s}-T_{i}(\mathbf{m})}{\sigma_{i}}\right]^{2},
$$

where $\mathrm{T}^{o b s}$ is the observed traveltime, $\mathrm{T}(\mathbf{m})$ is the corresponding synthetic traveltime for a model $\mathbf{m}$, and $\sigma$ is a measurement uncertainty weight (Tromp et al. 2005). For each iteration, misfit defined by Equation 3.2 is averaged over all windows for a given event, and for all events in a given iteration. The objective function for a given model $\mathbf{m}$ is defined as

$$
F(\mathbf{m})=\frac{1}{2 S} \sum_{s=1}^{S} \frac{1}{N_{s}} \sum_{i=1}^{N_{s}} \chi_{i}(\mathbf{m}),
$$

where $\mathrm{S}$ is the total number of sources, and $\mathrm{N}_{s}$ is the total number of windows for a given source s (Tape et al. 2010). Equation 4.1 is used as a measure for overall data-synthetic misfit for a given velocity model.

\subsubsection{Inverse problem, nonlinear optimization, regularization}

The objective of the inverse problem is to minimize the misfit function $(\mathrm{F}(\mathbf{m})$; Equation 4.1). In order to accomplish this, we calculate the gradient of the objective function for all source-receiver pairs using the adjoint-state method (Tarantola, 1984; Tromp et al., 2005; Fichtner et al., 2006a). In more classical tomography, the Hessian of the objective function, containing second derivatives, would be used in conjunction with the gradient to determine the location of the global minimum in the objective function (e.g. Woodhouse and Dziewonski, 1984; Ritsema et al., 1999). But with a complex, heterogeneous model, calculation of the Hessian is prohibitively expensive (Tape et al., 2007). Without the second derivative information contained in the Hessian, iterative methods taking advantage of nonlinear optimization algorithms can be used to search for the global minimum of the objective function using gradient information only.

In this study we use the nonlinear L-BFGS optimization algorithm (Liu and Nocedal, 1989) to calculate an optimal step length and search direction for model perturbation. This quasi-Newton method uses previous gradient calculations to construct curvature information and inform the current gradient calculation. The 
L-BFGS algorithm been shown to be the most computationally efficient choice in seismic inversion step length calculations (Modrak and Tromp, 2016), and has been specifically tested using synthetic inversions for realistic New Zealand sourcereceiver distributions (Section 3.4). At each iteration, gradient information is acquired using the adjoint-state method, and velocity model parameters $V_{p}$ and $V_{s}$ are updated. Density remained constant throughout the inversion, due to its limited sensitivity to the surface wave measurements used (Nazarian and Stokoe. 1984). Attenuation also remained constant, due to the use of a phase-only misfit function (Equation 3.2).

Source inversions can be undertaken alongside structural inversions, to improve locations and moment tensors (e.g. Zhu et al., 2015; Tao et al., 2018). Through manual review of initial synthetic waveforms, and careful selection of the initial event catalog (Section 3.2.2), we note the initial locations and moment tensors for our events are reasonable, and consequently we do not perform source inversions in this study. All waveform differences are therefore attributed to differences between a given velocity model $\mathbf{m}$, and true Earth structure.

Regularization is often used in tomographic inversions to prevent trapping of the optimization in local minima (Modrak and Tromp, 2016). As in the synthetic inversions of Section 3.4. we smooth the gradient by convolution with a Gaussian to suppress non-uniqueness in the updated models. Horizontal and vertical halfwidths are chosen larger than the expected spatial resolution of input data to promote resolution of large-scale features in early iterations. Smoothing is also used to reduce the effects of strong near-source and receiver perturbations that appear in the gradient due to the point approximation of sources and receivers in the spectral element mesh. Waveform bandpass and gradient smoothing length are reduced gradually throughout the inversion to conservatively approach the global minimum of the objective function. Selected parameters at various stages of the inversion are shown in Figure 4.3 , and detailed in Table F.1.

\subsubsection{Inversion legs}

We perform 28 L-BFGS iterations over six distinct inversion legs (Figure 4.3), and two mesh resolutions (Figure 4.2). The start of each new inversion leg is defined by selection of new time windows, and a change of input parameters including 
some or all of: waveform bandpass, windowing algorithm parameters, gradient smoothing length (Table F.1).

As is common in full-waveform tomography, we progress from low to high frequencies over the course of the inversion to ensure convergence towards the global minimum (e.g. Fichtner et al., 2009; Tape et al., 2010; Krischer et al., 2018). The initial leg inverts for longer-period waveforms (15-30 s) with a wide-radii gradient smoothing ( $\left.\sigma_{h}=20 \mathrm{~km} ; \sigma_{v}=10 \mathrm{~km}\right)$ to resolve very broad-scale features ( $>50 \mathrm{~km}$ ). Following inversion legs decrease either the shortest period of waveform data, the smoothing radii of the 3D Gaussian, or both (Figure 4.3). The final inversion leg inverts for long-period waveforms (4-30 s) with small corresponding smoothing radii $\left(\sigma_{h}=8 \mathrm{~km} ; \sigma_{v}=4 \mathrm{~km}\right.$ ) for higher resolution imaging (5-10 km resolution). New time windows were chosen at the beginning of each leg, and remained relatively consistent throughout a given leg to ensure that misfit assessment compared a similar segment of the dataset. Time windowing parameters were modified slightly at each leg to reflect changes in input data (Table A.1).

The choice to begin a new inversion leg was motivated primarily by the behavior of the nonlinear optimization algorithm. Loss of descent direction, negligible misfit reduction, or a large number of line search step counts $(>5)$, were all taken into account in deciding to terminate a given inversion leg (Table F.1). New legs did not necessarily correspond to restart conditions in the optimization algorithm. The aforementioned factors were taken as indicators of numerical stagnation, and parameter changes were introduced to refresh or stimulate the optimization procedure. At the start of each leg, multiple trial iterations were performed with variations in windowing parameters, bandpass, and gradient smoothing length to determine a suitable set of parameters for the following leg. Selected trials retained a similar number of measurements, and waveform misfit, compared to the previous iteration.

A change of mesh was required between inversion legs D and E (Figure 4.3) to accommodate high-frequency waveforms. Due to differing mesh constructions (Figure 4.2, the change required interpolation of the velocity model from the coarse, irregular, spectral element mesh, onto a regular grid, and subsequent interpolation from the regular grid onto the fine-resolution irregular mesh. This method saved roughly $400000 \mathrm{CPU}$-hours by allowing us to perform the initial four inversion 


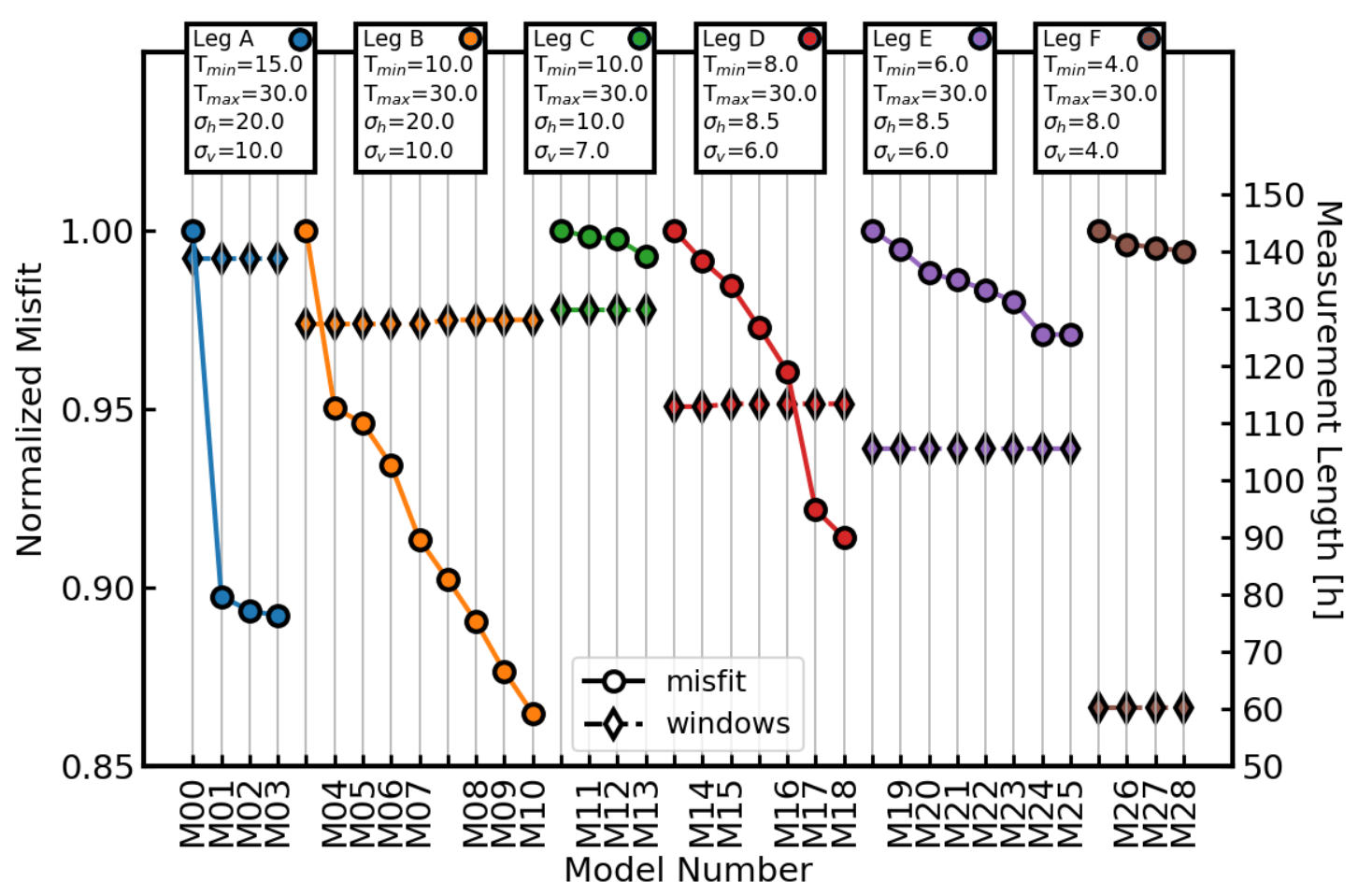

Figure 4.3: Convergence plot showing total misfit reduction over the course of the inversion. Each line represents an individual inversion leg, labelled A-F. Bandpass (T), and horizontal and vertical standard deviation of the 3D Gaussian used for gradient smoothing $(\sigma)$ annotated above each leg. Misfits plotted as circles, normalized to the starting misfit of any given inversion leg. Diamonds show cumulative window length in hours. Adjacent points that share a model number (e.g. at the transition between inversion legs) correspond to re-evaluation of the misfit using the same model, e.g. through selection of new windows, or a change of inversion parameters.

legs on a low-resolution mesh. As a result of this interpolation, mesh artefacts are visible at depths corresponding to doubling and tripling layers in the coarse resolution mesh (Figure $4.4 \mathrm{E}$, I). These artefacts arose from nearest-neighbor interpolation of the irregular grid, however they are visible only in regions with little to no resolution (e.g. southeast of Hikurangi trench), do not affect waveform propagation simulations, and are therefore deemed irrelevant for our interpretations.

\subsection{Inversion results}

We present our final velocity model, M28, alongside comparisons with the initial velocity model, M00 (Figure 4.4). Model differences are shown in terms of net 
model update $\ln \left(\mathrm{m}_{28} / \mathrm{m}_{00}\right)$, which to first order approximates the percentage difference $\left(\mathrm{m}_{28} / \mathrm{m}_{00}-1\right)$ but more reliably represents model differences over a wide range of values (Tape et al., 2007). In the following section we discuss overall velocity changes in terms of absolute $\mathrm{V}_{s}$, and the $\mathrm{V}_{p} / \mathrm{V}_{s}$ ratio (Fig 4.7). Waveform improvement for select source-receiver pairs (Figure 4.8), and in bulk (Figure 4.9), are also discussed.

\subsubsection{Velocity changes}

The final velocity model, M28, shows large-scale, heterogeneous velocity changes with respect to initial values. Figure 4.4 shows $V_{s}$ comparisons at 5, 15, and $25 \mathrm{~km}$ depth, respectively. The maximum velocity changes defined by $\ln \left(\mathrm{m}_{28} / \mathrm{m}_{00}\right)$ are +0.33 for $\mathrm{V}_{s}$ (Figure $4.4 \mathrm{C}$ ) and +0.25 for $\mathrm{V}_{p}$ (Figure E.1). The most striking heterogeneities are visible at mid-crustal depths (15 km, Figure $4.4 \mathrm{D}-\mathrm{F})$ with velocity models appearing very similar below $25 \mathrm{~km}$ (Figure $4.4 \mathrm{G}-\mathrm{I}$ ). In general, the final model is characterized by slower wavespeeds almost everywhere, with specific areas requiring substantially increased velocities. Most of these changes do not introduce new features, but rather serve to modify existing velocity features through changes in wavespeed, as well as sharpening and shifting of spatial boundaries.

At $5 \mathrm{~km}$ depth (Figure 4.4A), large scale features $(>50 \mathrm{~km}$ ) like the low-velocity accretionary wedge, the high-velocity axial ranges, and the low-velocity Taranaki and Whanganui basins (Fig 1.1), are identifiable in both initial and final models. We expect these large-scale similarities, as the longest waveform period of $30 \mathrm{~s}$ corresponds to spatial resolutions less than $100 \mathrm{~km}$ (for an average velocity of $3 \mathrm{~km} \mathrm{~s}^{-1}$ ). On average, velocity changes range in values from $\pm 5-20 \%$ in the top $30 \mathrm{~km}$ (Figures 4.5 and 4.6), with velocity changes above $\pm 1 \%$ down to $50 \mathrm{~km}$ depth. Below approximately $100 \mathrm{~km}$ depth, the initial and final models are the same due to the limited depth sensitivity of 30 s surface waves. As expected, in regions with sparse data coverage (i.e. deep ocean, offshore the west coast of the North Island), recovered velocity changes are negligible. In the remainder of this section we discuss wavespeed changes that have introduced features previously unseen in the initial model.

Consecutive net model updates at $5 \mathrm{~km}$ depths are shown in Figure 4.5, corresponding to the final iteration of each inversion leg (Figure 4.3). This provides a 


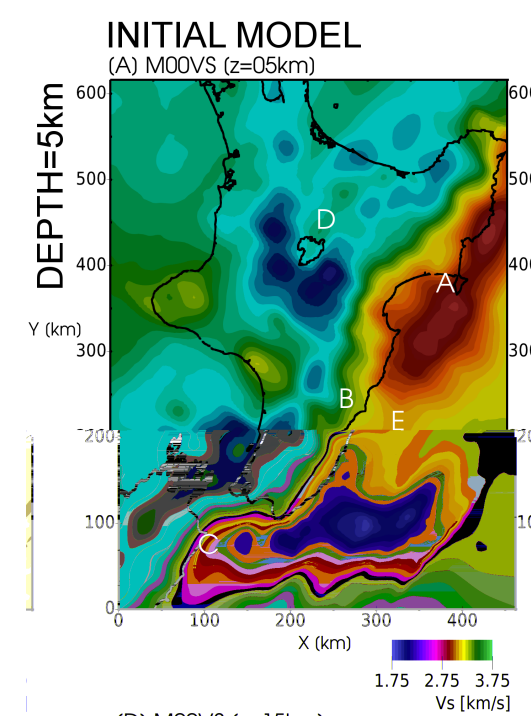

FINAL MODEL

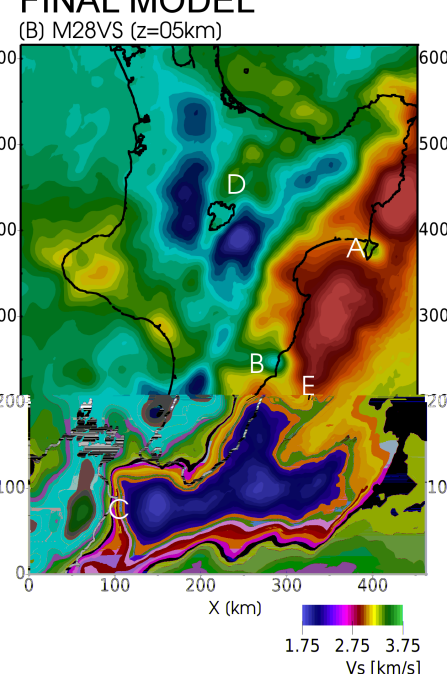

UPDATE
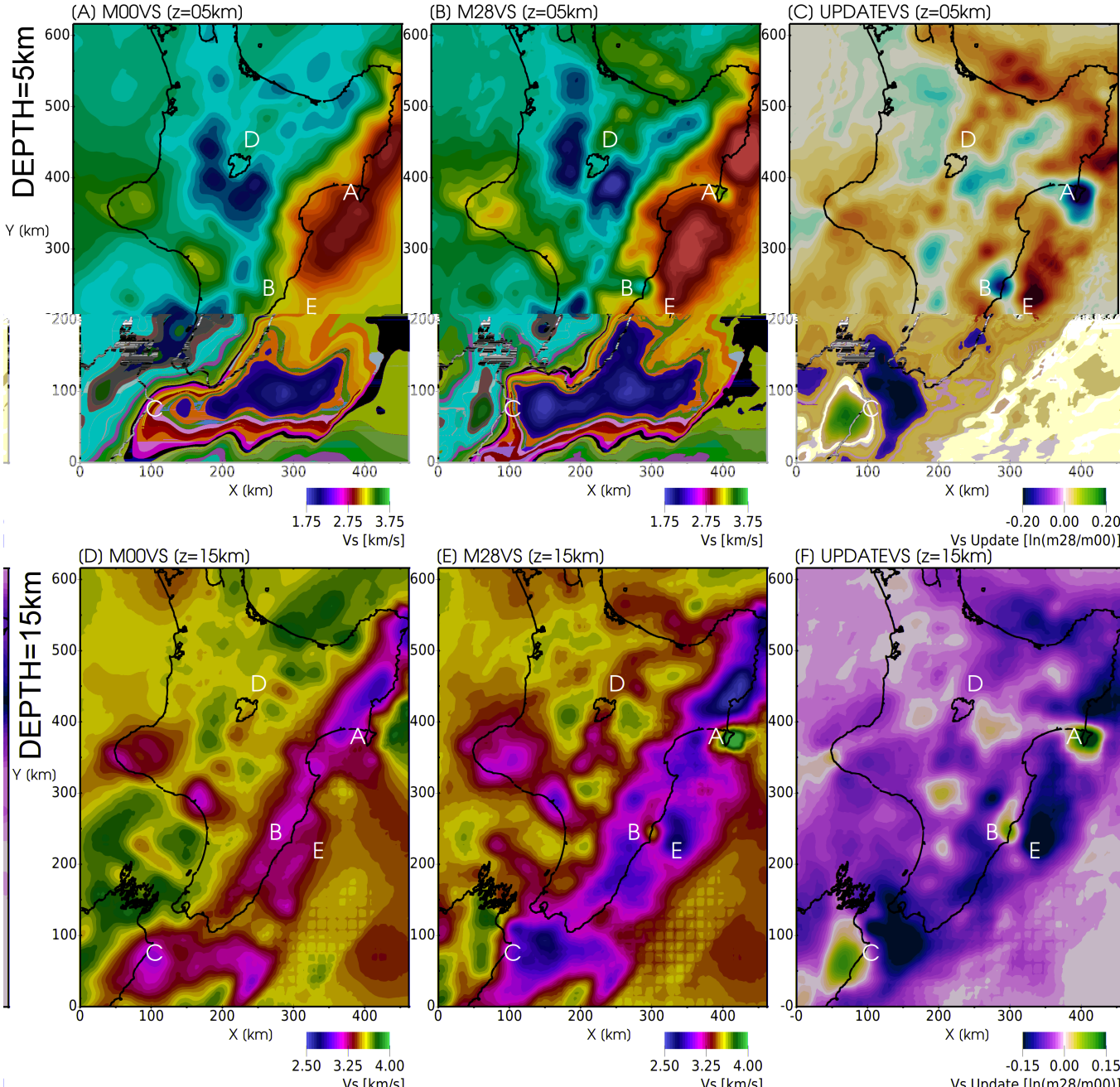

(F) UPDATEVS $(z=15 \mathrm{~km})$
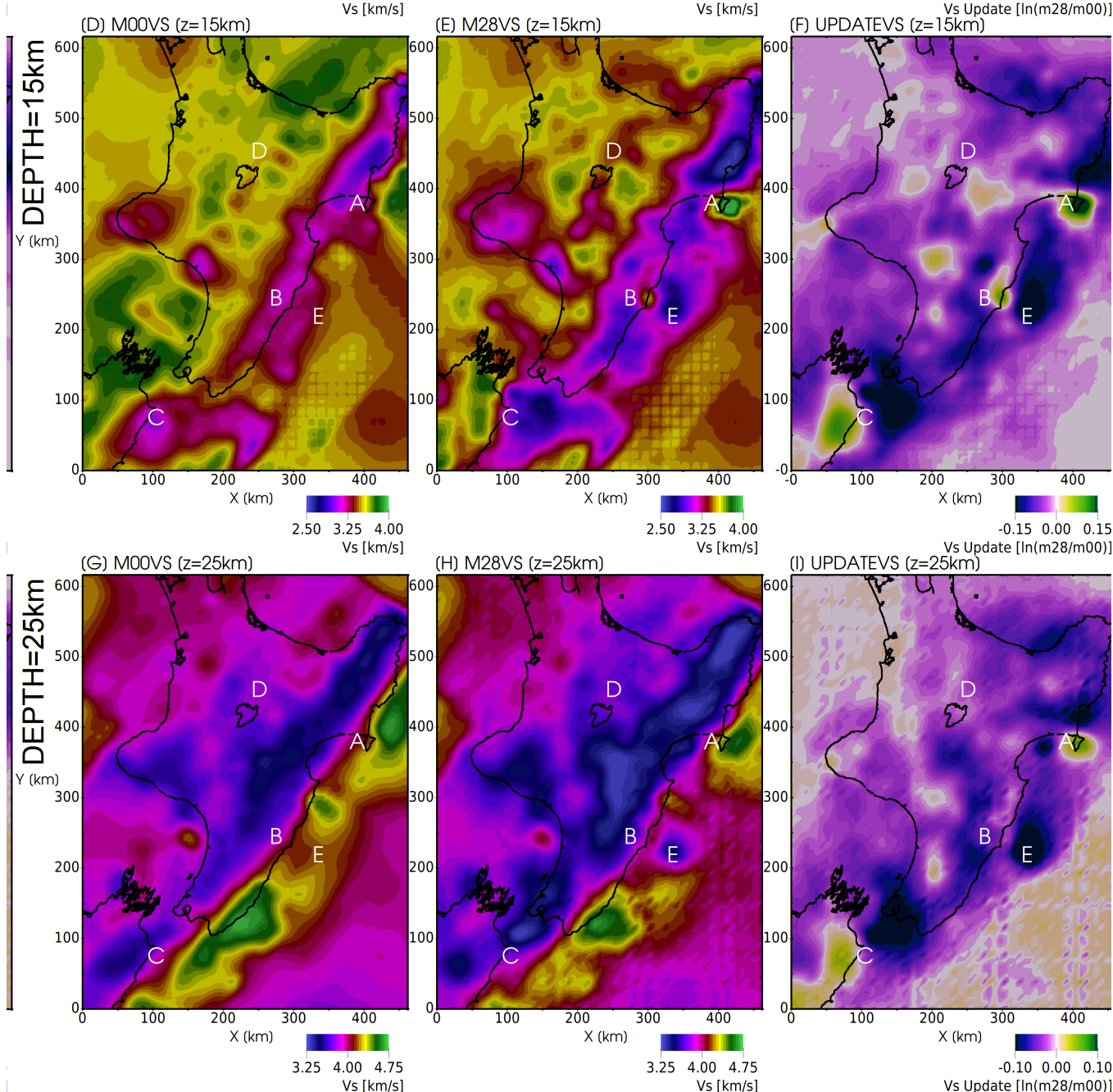

Figure 4.4: Comparisons of M00 and M28 $\mathrm{V}_{s}$ velocity models at various depth slices. Columns represent initial model M00 (left), final model M28 (center), and net model update $\ln (\mathrm{m} 28 / \mathrm{m} 00$ ) (right), while rows represent depth slices at $5 \mathrm{~km}$ (top), $15 \mathrm{~km}$ (middle), and $25 \mathrm{~km}$ (bottom). Annotated letters A-E relate to features discussed in Section 4.4 Note the differing color scales between rows and columns. Numerical artefacts are visible in D, E, F, I, J, discussed in Section 4.3.3 
qualitative look at model changes for each bandpass. As expected, the initial resolution of long-wavelength structure, evident in Figure 4.5A, gradually brings out increased detail (Figure $4.5 \mathrm{~B}-\mathrm{F}$ ). By inversion leg C (Figure $4.5 \mathrm{C}$ ), it is possible to make out most of the large scale features (features A-E) which become the basis for the most striking velocity changes seen in the M28 $\mathrm{V}_{s}$ model (Figure 4.4). With decreasing bandpass, detail increases, however large scale features do not change considerably past inversion leg C. It is interesting to note that resolution of features $\mathrm{A}-\mathrm{E}$ are already visible by the second inversion leg (Figure $4.5 \mathrm{~B}$ ), suggesting that these features were necessary to fit the initial long-wavelength data-synthetic misfit. For the most part, the last two inversion legs (Figure $4.5 \mathrm{E}, \mathrm{F}$ ) do not introduce drastic changes, but rather serve to sharpen existing features and increase detail, owing to the smaller waveform bandpass and decreased smoothing lengths used. Feature D, related to the TVZ, is one area that does show increasing detail after inversion leg $\mathrm{E}$ (Figure $4.5 \mathrm{E}$ ), and resolves the unique velocity anomalies seen in the final M28 $\mathrm{V}_{s}$ model (Figure 4.4).

Introduced heterogeneity at $5 \mathrm{~km}$ depth is dominated by three, strong ( $>20 \%$ ) velocity increases, labelled A, B and C in Figure 4.4. These perturbations introduce fast velocity anomalies in the forearc region, visible directly beneath A) Māhia Peninsula, B) Pōrangahau, and C) the northern South Island. High velocities related to feature $\mathrm{C}$ are bounded to the northwest by a strong velocity gradient with slow velocities recovered in the Cook Strait (Figure $4.4 \mathrm{~B}$ ). In the central North Island, at $5 \mathrm{~km}$ depth, slow velocities in the TVZ (feature D) are bounded by high velocities to the east and west (Figure $4.4 \mathrm{~B}$ ). This strong gradient is shallow and no longer seen at the $15 \mathrm{~km}$ depth slice (Fig $4.4 \mathrm{E})$.

Offshore Pōrangahau, feature E is a localized velocity decrease visible at each depth slice (Figure $4.4 \mathrm{~B}, \mathrm{E}, \mathrm{I})$. Feature $\mathrm{E}$ is most visible as a strong low-velocity anomaly at $25 \mathrm{~km}$ depth (Fig 4.4 ). In absolute $\mathrm{V}_{s}$, the positive perturbations below feature A (Māhia Peninsula) and feature B (Pōrangahau) are resolved as roughly point-local velocity anomalies (Figure $4.4 \mathrm{~B}$ ). These anomalies are visible down to $15 \mathrm{~km}$ depth (Figure $4.4 \mathrm{E}$ ). Similarly in Cook Strait, the strong $\mathrm{V}_{s}$ gradient related to feature $C$ is visible down to $15 \mathrm{~km}$ depth (Figure $4.4 \mathrm{E}$ ). At $25 \mathrm{~km}$ depth (Fig $4.4 \mathrm{I}$ ), large-scale features are characterized by the plate interface region, with the highvelocity Pacific plate contrasting against the slower relative velocities of the upper, Australian, plate. The most striking difference here is the low-velocity feature E. 

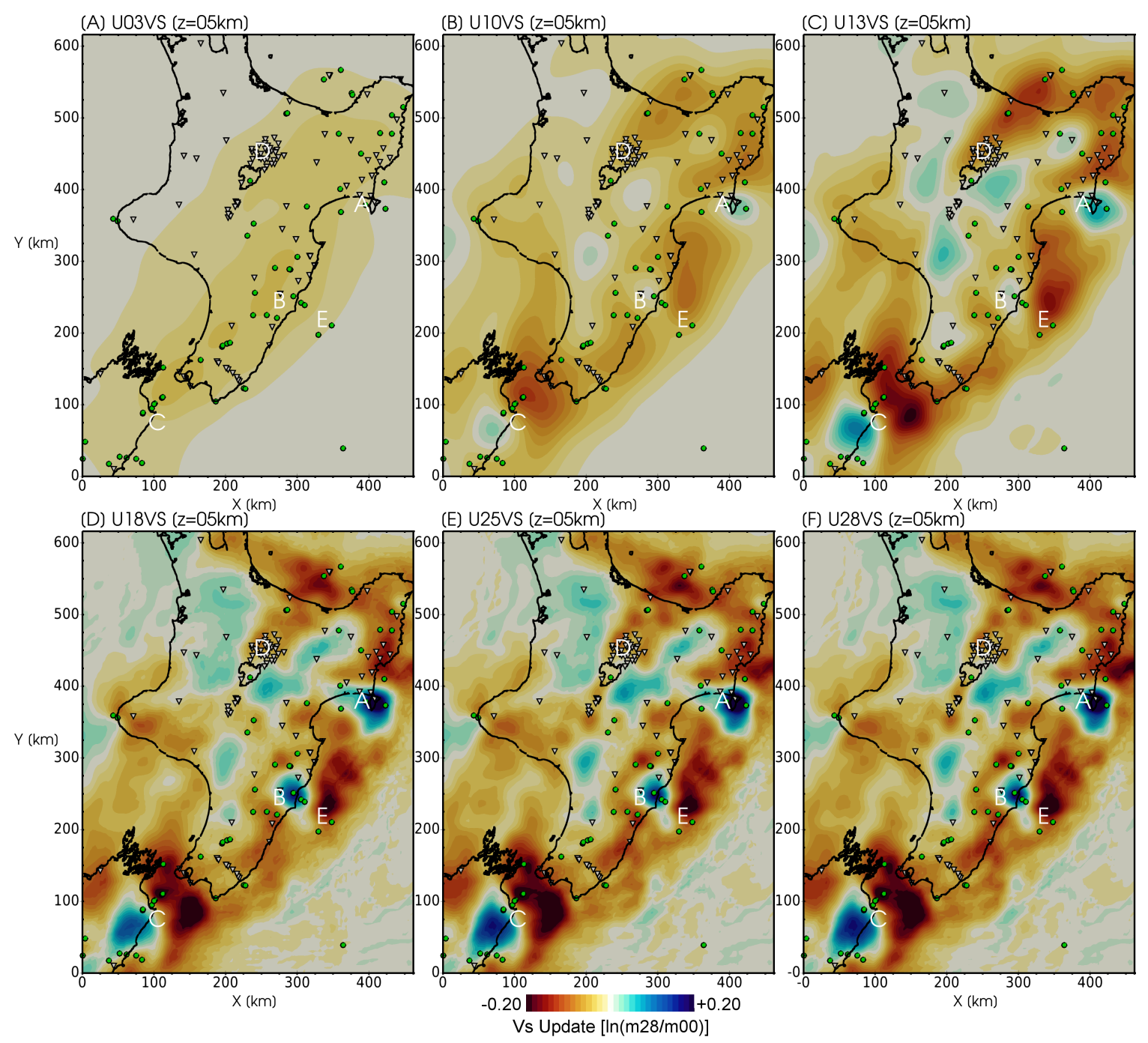

Figure 4.5: $\mathrm{V}_{s}$ net model update at $5 \mathrm{~km}$ depth slices for the final iteration of each inversion leg (Figure 4.3). Features A-E are the same as in Figure 4.4. Color scale is the same for each figure, at \pm 0.20 (approximately $\pm 20 \%$ velocity change), however maximum velocity changes by M28 are up to $\pm 33 \%$. 

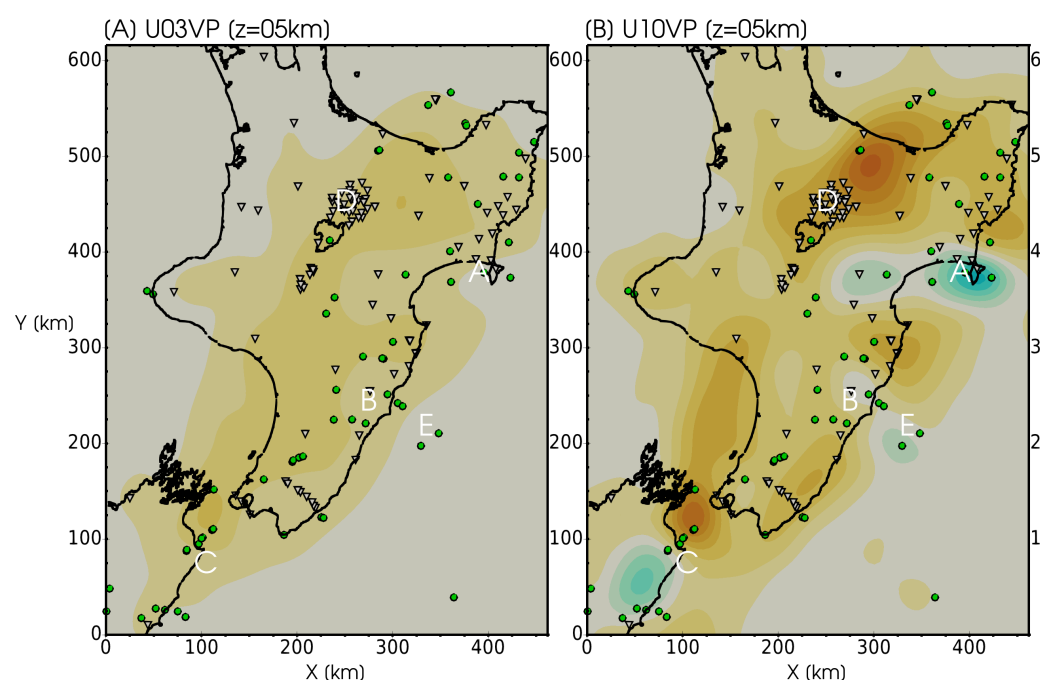

(C) U13VP (z=05km)
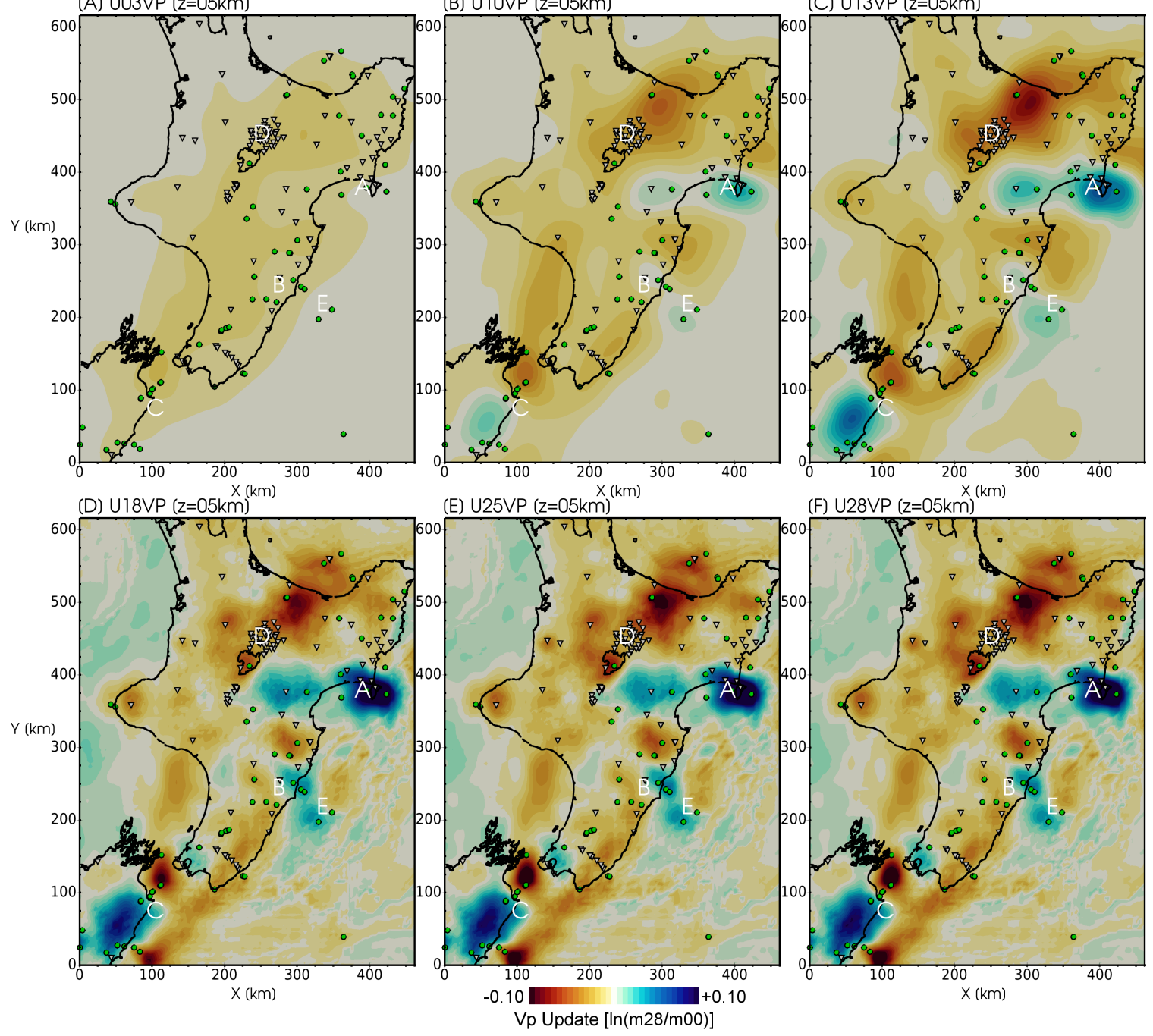

(F) U28VP (z=05km)

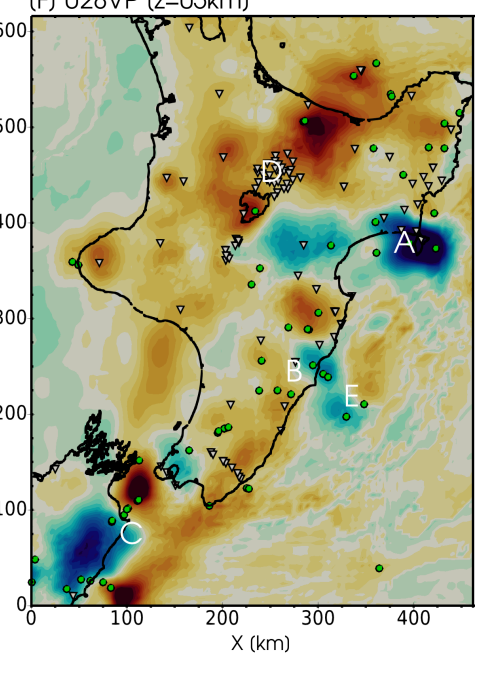

Figure 4.6: $\mathrm{V}_{p}$ net model update at $5 \mathrm{~km}$ depth slices for the final iteration of each inversion leg (Figure 4.3). Features A-E are the same as in Figure 4.4. Color scale is the same for each figure, at \pm 0.10 (approximately $\pm 10 \%$ velocity change), however maximum velocity changes by M28 are up to $\pm 25 \%$. 
The ratio of seismic velocities $\left(\mathrm{V}_{p} / \mathrm{V}_{s}\right)$ is often used in tomographic studies in conjunction with interpretations of absolute velocity; high $\mathrm{V}_{p} / \mathrm{V}_{s}$ has been observed to correlate with increased clay content in sedimentary rocks, increased porosity, highly fractured rocks, and increased fluid pressures (Eberhart-Phillips et al., 1989. 2005; Audet et al., 2009). To derive $\mathrm{V}_{p} / \mathrm{V}_{s}$ we directly divide our $\mathrm{V}_{p}$ model by our $\mathrm{V}_{s}$ model. With increased sensitivity to $\mathrm{V}_{s}$ structure through the dominant measurement of surface waves, we see dramatic changes in the recovered M28 $V_{p} / V_{s}$ model at $5 \mathrm{~km}$ depth (Figure 4.7). One thing to note is that the net model update of $\mathrm{V}_{s}$ (Figure 4.4 $\mathrm{C}$ ) and $\mathrm{V}_{p} / \mathrm{V}_{s}$ ratio (Figure 4.7C) show strong similarities, hinting that the resolved differences in $\mathrm{V}_{p} / \mathrm{V}_{s}$ are more related to changes in $\mathrm{V}_{s}$ structure, with weaker contributions from updates in $\mathrm{V}_{p}$ (Figure E.1). Although $\mathrm{V}_{s}$ and $\mathrm{V}_{p}$ both show velocity change (Figures 4.5 and 4.6), we must be cautious when interpreting $\mathrm{V}_{p} / \mathrm{V}_{s}$ derived from direct division, as apparent $\mathrm{V}_{p} / \mathrm{V}_{s}$ structures may arise from differences in resolution of $\mathrm{V}_{p}$ and $\mathrm{V}_{s}$ within our inversion.

Very large-scale structure $(>100 \mathrm{~km})$ can be correlated between initial and final models, with high $\mathrm{V}_{p} / \mathrm{V}_{s}$ in the forearc region, and low $\mathrm{V}_{p} / \mathrm{V}_{s}$ in the back-arc (Figure 4.7A, B). However, the final model introduces new, strong, $\mathrm{V}_{p} / \mathrm{V}_{s}$ heterogeneities. Features $A-E$ show corresponding signatures in $\mathrm{V}_{p} / \mathrm{V}_{s}$ (Figure 4.7). The high-velocity anomalies below A) Māhia Peninsula, and B) Pōrangahau, are characterized by localized, low $\mathrm{V}_{p} / \mathrm{V}_{s}$ (Figure 4.7B). Additionally, the strong velocity gradient observed in the Cook Strait (Figure $4.4 \mathrm{~B}$, feature $\mathrm{C}$ ) is also visible in $\mathrm{V}_{p} / \mathrm{V}_{s}$ (Figure 4.7B), as low $\mathrm{V}_{p} / \mathrm{V}_{s}$ in the northern South Island bounded to the northeast by low $\mathrm{V}_{p} / \mathrm{V}_{s}$ in the Cook Strait. In the central North Island (feature $\mathrm{D}$ ) the low $\mathrm{V}_{s}$ velocities (Figure $4.4 \mathrm{~B}$ ) in the TVZ are characterized by high $\mathrm{V}_{p} / \mathrm{V}_{s}$, bounded by low $\mathrm{V}_{p} / \mathrm{V}_{s}$ structures on all sides (Figure 4.7B). $\mathrm{V}_{p} / \mathrm{V}_{s}$ shows similar heterogeneity below $5 \mathrm{~km}$ depth, which becomes less significant below $25 \mathrm{~km}$ depth (Figure E.2).

\subsubsection{Waveform improvement}

Waveforms show considerable improvement, but data-synthetic misfits remain in the final velocity model M28 (Figure 4.8). Here we discuss waveforms at 6-30 s to emphasize longer-period surface wave signals, as waveforms at 4-30 s begin to show lower signal-to-noise ratios and are therefore less illustrative of waveform improvement. Figure 4.8 compares initial and final velocity model synthetics for 8 


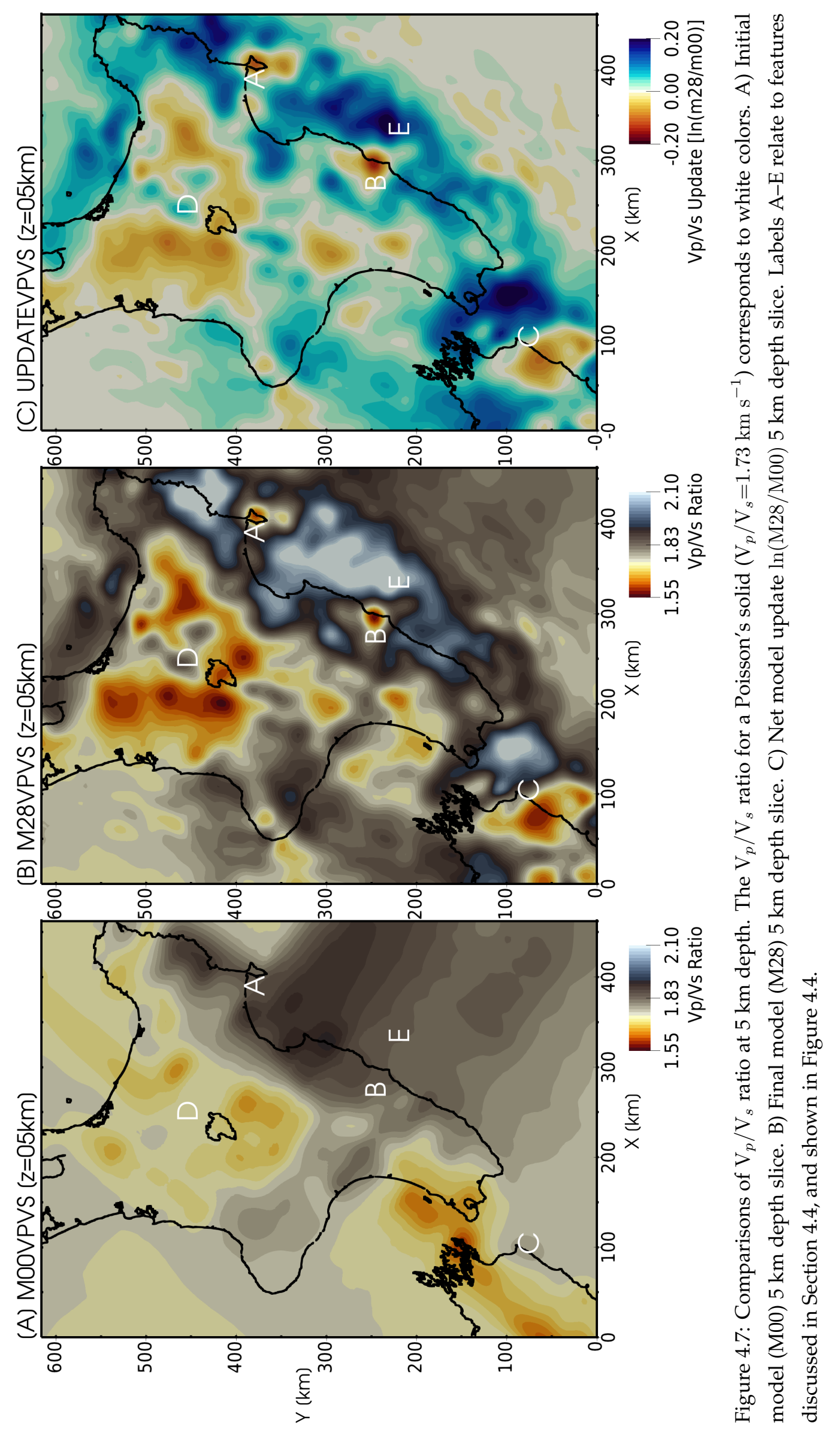


source-receiver pairs, each meant to be representative of the larger dataset. Direct arrivals are well fit by the initial model, which is expected, since it was derived using body-wave traveltime tomography (e.g. Figure 4.8A). Surface waves and later arrivals in the initial model show considerable time shift with respect to the data at this bandpass (e.g. Figure $4.8 \mathrm{~B}, \mathrm{C}$ ). Paths which pass through relatively simple crustal structure (e.g. Figure $4.8 \mathrm{E}-\mathrm{H}$ ) show good initial waveform fit, while seismic waves that travel through more complex tectonic regions such as the lowvelocity accretionary wedge (e.g. Figure $4.8 \mathrm{~B}-\mathrm{D}$ ) have large initial misfit. The high initial misfit in the offshore region (Figure 4.8D) shows the limited accuracy of the initial model away from land.

After the inversion, long-period ( $>10 \mathrm{~s}$ ) time shifts are reduced to $<1 \mathrm{~s}$ and surface waves for all waveform shown are mostly fit, but high-frequency components show varying degrees of misfit (Figure $4.8 \mathrm{E}$ ). Some synthetics show increased high-frequency components not seen in data (Figure 4.8C). Amplitude information, which was not inverted for, shows little to no improvement; in most cases amplitude differences between data and synthetics do not change. Errors in higher frequencies and amplitudes might be attributable to inaccuracies in the underlying attenuation model. The attenuation models were not updated during the inversion, hence velocity changes alone were not enough to fit the phases of high frequencies. Similarly, coda waves are left mostly unfit (e.g. Figure $4.8 \mathrm{E}$ ), hinting at the difficulty of accurately resolving smaller-scale heterogeneities, scatterers, and basin effects arising from sharp impedance contrasts (e.g. Kaneko et al., 2019).

\subsubsection{Bulk misfit assessment}

Total misfit is reduced considerably throughout the inversion. The convergence plot in Figure 4.3 shows the reduction in overall normalized misfit (Equation 4.1) over 28 iterations. The largest relative reductions in misfit occur in the initial two inversion legs (A, B), at the 15-30 s and 10-30 s bandpasses. Total window length is also maximum here at $500000 \mathrm{~s}$ or $138 \mathrm{~h}$, occupying approximately $30 \%$ of the total dataset (total dataset $\approx 450 \mathrm{~h}$; or 1800 3-component waveforms with $300 \mathrm{~s}$ seismogram length). At Leg $C$, an attempt to reduce gradient smoothing while retaining bandpass was made, but negligible misfit reduction suggests that the previous inversion leg B was capable of fitting the 10-30 s period range. At 8-30 s 

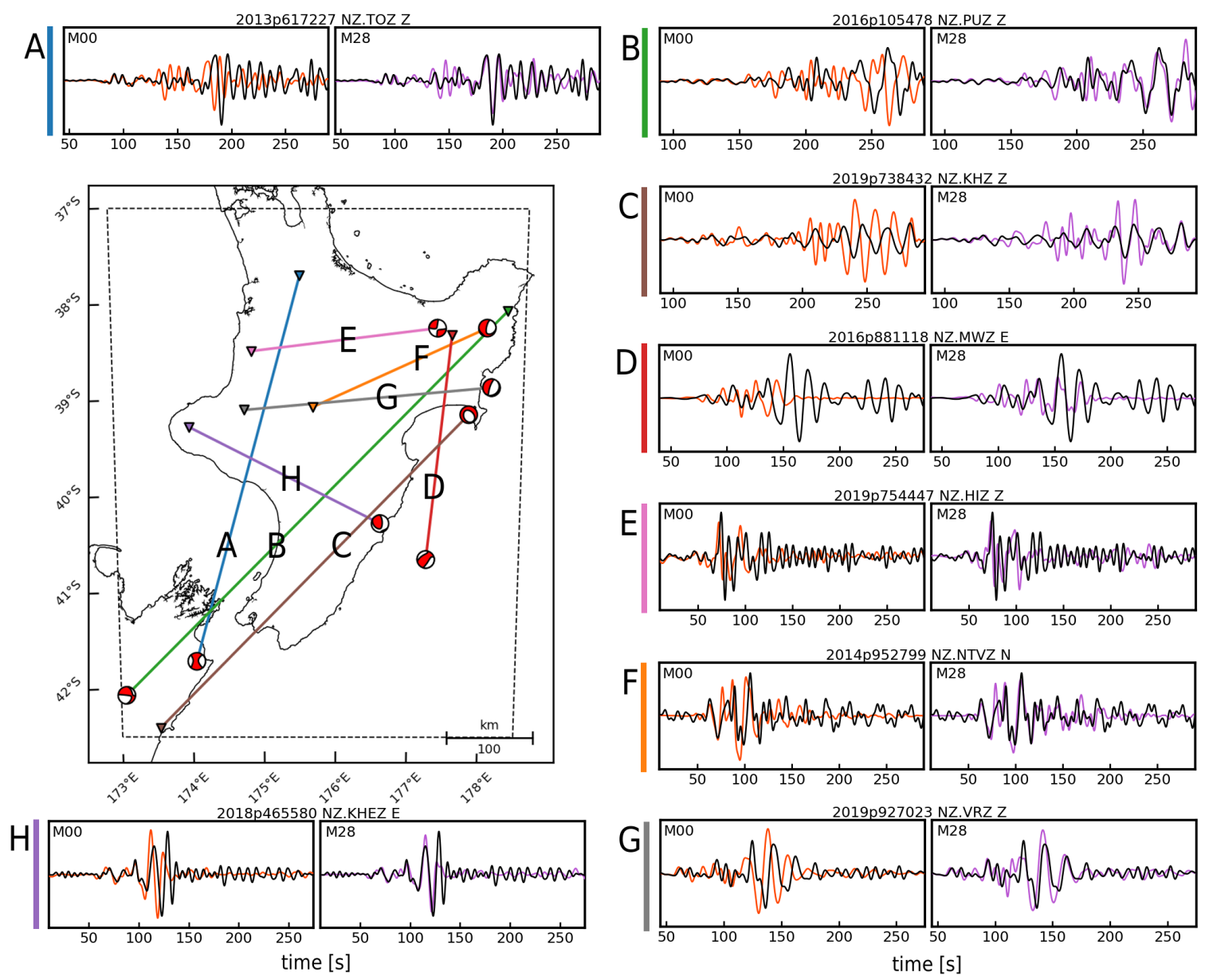

Figure 4.8: Waveform comparisons for eight unique source-receiver pairs. Each comparison (A-H) consists of two panels showing data (black) plotted against the initial model (M00) synthetics in red (left panels), and final model (M28) synthetics in purple (right panels), processed and filtered identically at $6-30$ s periods. Map shows corresponding moment tensors, receiver locations, and connecting raypaths. GeoNet event ID, station code, and waveform component annotated in the title of each panel. Y-axes in units of displacement $[\mathrm{m}]$. 
(Leg D) another large decrease in overall misfit was seen. By 6-30 s (Leg E) the behavior of the misfit reduction is more steady and less pronounced than earlier inversion legs.

For long-period inversion legs (E, F), signal-to-noise ratio increased as filter periods include signals from the secondary microseism (5-10 s), where ocean-generated noise becomes a more dominant feature in waveforms (Webb, 1998). This can be seen in the large decrease in total measurements for the 4-30 s period band, which occupies approximately $200000 \mathrm{~s}$ or $60 \mathrm{~h}$, corresponding to $15 \%$ of the total dataset. After the 28th iteration, a number of trial iterations were run on an ultra-fine resolution mesh, accurate down to $2 \mathrm{~s}$ with roughly 930000 elements. At these short periods, observed waveform signal-to-noise ratio increased dramatically, and misfit reduction was negligible with respect to previous inversion legs. At this point, a decision was made to terminate the inversion.

Histograms comparing time shifts and amplitude differences between initial and final models are shown in Figure 4.9. Amplitude differences here are defined by Equation 3.1 as $\Delta \ln A=\ln \left[\int d^{2}(t) d t / \int s^{2}(t) d t\right]$, where $d$ and $s$ are observed and synthetic waveforms, respectively. 6-30 s waveforms were generated and measured using the M00 and M28 models for the 60 events used in the inversion (Figure $4.9 \mathrm{~A}, \mathrm{~B}$ ), as well as 60 events from a separate post-hoc validation catalog (Figure 4.9 C, D). The inversion histograms show that the initial model had a mean and standard deviation time shift of $2.0 \pm 3.9 \mathrm{~s}$ which was reduced to $0.5 \pm 3.7 \mathrm{~s}$ by the final model, showing that overall waveform time shifts were improved throughout the inversion. Amplitude differences show negligible change between initial and final models, which is expected as we use a phase-only misfit function that does not incorporate amplitude information. The post-hoc validation catalog was comprised of 60 events not used in the inversion (Table F.3). These histograms show similar behavior to the inversion results, suggesting that the model improvements were not made only for events included in the dataset, but that overall improvement of the velocity model has led to resulting improvement of data not included in the inversion. 

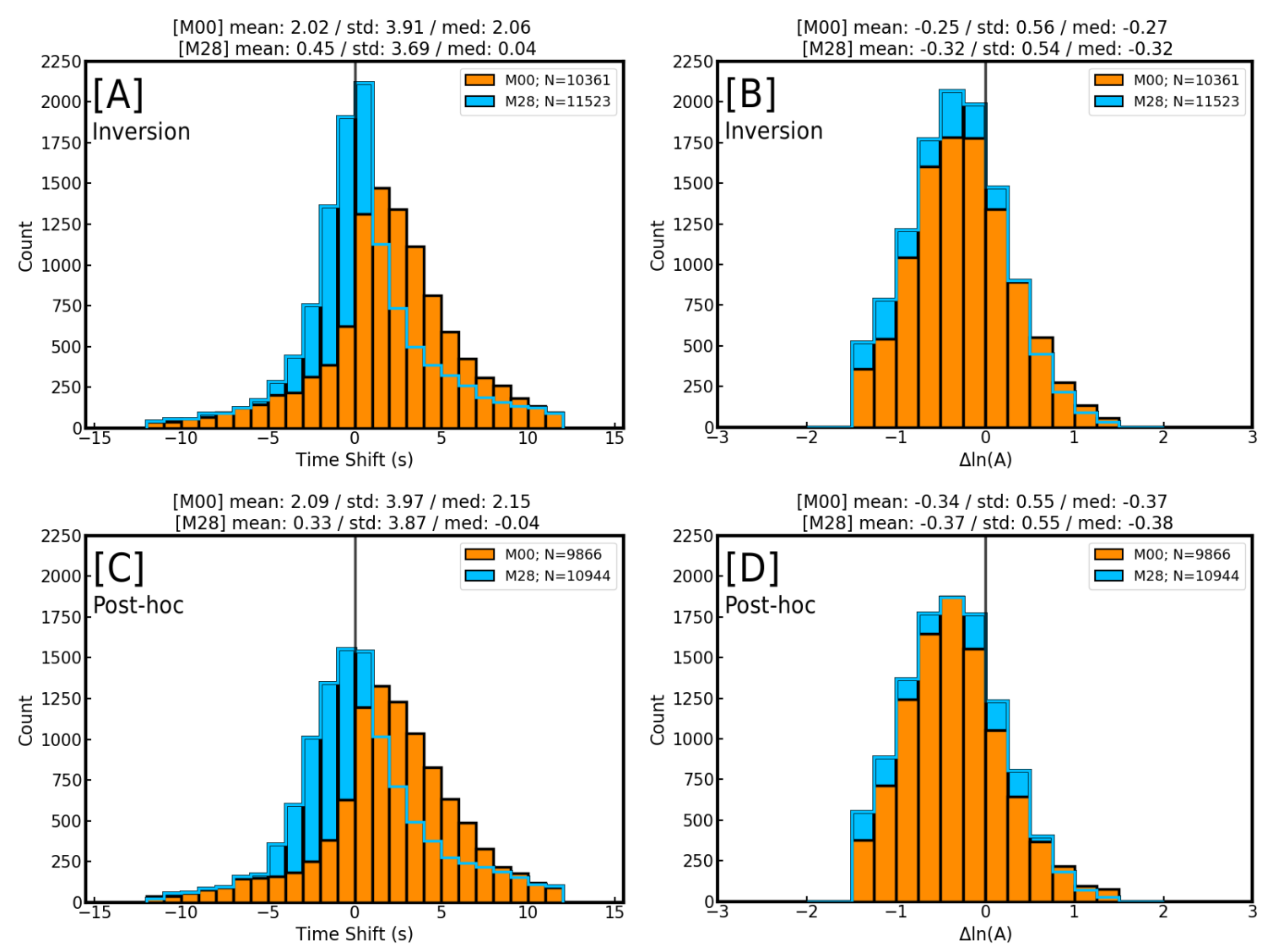

Figure 4.9: Misfit histograms detailing bulk misfit assessment between the initial model M00 (orange), and final model M28 (blue) for 60 events used in the inversion (top) and a 60 event post-hoc validation catalog (bottom). A) Time shift for 6-30 s waveforms evaluated using the initial and final models. B) Amplitude difference (Equation 3.1) for events used in the inversion. C) Time shift histogram for post-hoc validation events. D) Amplitude difference for post-hoc validation events. Mean, standard deviation, and median values for each respective histogram given in the title of each figure. Number of measurements for each histogram provided in the respective legends. 


\subsubsection{Computational expense}

The total inversion required approximately 500000 CPU-hours. Forward simulations on the coarse resolution mesh required $0.5 \mathrm{~h}$ on 40 physical cores of Māui, while the fine resolution mesh required $0.75 \mathrm{~h}$ on 80 physical cores. For each iteration, 60 forward simulations and 60 adjoint simulations were required to generate synthetics and gradient, respectively. For any given iteration, an additional $60 \times \mathrm{N}$ forward simulations were required for the optimization line search, to determine an acceptable perturbation amplitude to minimize the objective function. Here, $\mathrm{N}$ is the total number of trial steps required for the nonlinear optimization algorithm to determine an acceptable step length. $\mathrm{N}$ typically ranged between 1 and 3 (Table F.1). If $\mathrm{N}$ reached values $>5$, considerations were made to begin a new inversion leg. Gradient smoothing was performed in parallel once per iteration, while waveform preprocessing and misfit quantification were run in serial and required less than $2000 \mathrm{CPU}$-hours for the entire inversion.

\subsection{Resolution analysis}

Resolution information from tomographic inversions is important if velocity models are to be used for interpretations of Earth structure and tectonic processes. Small resolved features and sharp gradients do not automatically translate to improved resolution, and comparisons to external studies or known tectonic or geologic features are not as robust without supporting estimations of model resolution. However, traditional tomographic model assessment through methods such as analysis of posterior covariance and resolution matrices (e.g Tarantola. 2005; Nolet, 2008) are computationally infeasible with large, heterogeneous velocity models.

Point spread functions, which are a measure of how much a point-localized perturbation is smeared, or blurred, by the inversion procedure, can be used to assess image quality in full-waveform inversion (e.g. Fichtner and Leeuwen, 2015; Zhu et al., 2015; Tao et al., 2018). Calculating the action of the Hessian on a model perturbation has been shown as an elegant method for calculating an approximate point spread function (Fichtner and Leeuwen, 2015). Here we use a finite- 
difference approximation to calculate

$$
\mathbf{H} \delta \mathbf{m} \approx \mathbf{g}(\mathbf{m}+\delta \mathbf{m})-\mathbf{g}(\mathbf{m})
$$

where $\mathbf{H}$ denotes the Hessian and $\delta \mathbf{m}$ is a local model perturbation with respect to the initial model. If the final velocity model is close to converged, such that the curvature of the objective function matches the curvature around the global minimum, the "action Hessian" (Equation 4.2) acts as a conservative estimate of the point spread function (Fichtner and Trampert, 2011a b; Fichtner and Leeuwen, 2015). Zhu et al. (2015) note that the finite difference scheme used, as well as the sign and amplitude of perturbations $\delta \mathbf{m}$, leads to comparable results.

Careful choice of the input perturbation $\delta \mathbf{m}$ allow the action Hessian to be a powerful method for assessing model resolution. We use similar notation as Fichtner and Leeuwen (2015) to denote point spread functions in the form $\mathrm{V}_{X Y}$, where $X$ defines the quantity in which the perturbation is made $\left(\mathrm{V}_{p}\right.$ or $\left.\mathrm{V}_{s}\right)$, and $Y$ denotes the recovered point spread function. For example $V_{S S}$ refers to a $V_{s}$ point spread function for a perturbation in $\mathrm{V}_{s}$, whereas $\mathrm{V}_{P S}$ quantifies parameter trade-offs, and shows the effect of $a V_{p}$ perturbation on $V_{s}$ recovery.

\subsubsection{Point spread function volumes}

We calculate a volume of point spread functions for homogeneous, constant-velocity perturbations following the methods of Fichtner and Leeuwen (2015). Resulting point spread function volumes have high amplitudes in areas where heterogeneities have a strong effect on waveform misfit. The action of the Hessian on a homogeneous volume of $100 \mathrm{~m} \mathrm{~s}^{-1} \mathrm{~V}_{s}$ perturbation is shown as $\mathrm{V}_{S S}$ and $\mathrm{V}_{S P}$ in Figure 4.10. The resulting volume of point spread functions is smoothed with a 3D Gaussian $\left(\sigma_{h}=6 \mathrm{~km} ; \sigma_{v}=2 \mathrm{~km}\right)$ to remove high-frequency components that are not suitable for interpretations of our velocity model.

We recover large amplitudes and heterogeneous coverage in $\mathrm{V}_{S S}$ relating to areas of the model in which $\mathrm{V}_{s}$ heterogeneities have significant impact on the misfit (Figure 4.10A, B, E, F). As expected, areas with high ray density (Figure 4.1B) correspond to regions with high amplitudes of $\mathrm{V}_{S S}$. Regions immediately surrounding stations, most visible in the western North Island, show high $\mathrm{V}_{S S}$ amplitudes, hinting at the strong control of near-source and station velocity structure on misfit (Figure 4.10 C). As noted before, areas with no data coverage, such as the far 
offshore region to the southeast, show negligible sensitivity (Figure 4.10A).

At depth, resolution decreases and is confined mostly to the forearc region (Figure $4.10 \mathrm{~B}$ ). This is better visualized in cross sections (Figure 4.10 $\mathrm{E}, \mathrm{F}$ ), which show the strongest sensitivity in the upper $25 \mathrm{~km}$ (Figure $4.10 \mathrm{E}$ ). Amplitudes are visible down to depths of $75 \mathrm{~km}$, related to the vertical sensitivity of the longest-period (30 s) surface waves used. An east-west cross section (Figure 4.10F) shows amplitudes are largest in the eastern North Island and confined to depths above $25 \mathrm{~km}$ for the western North Island.

Parameter trade-off is quantified in $\mathrm{V}_{S P}$, which shows the effect of $\mathrm{V}_{s}$ perturbations on $\mathrm{V}_{p}$ recovery (Figure 4.10 C, D). $\mathrm{V}_{S P}$ shows that some parameter trade-off occurs in the shallow crust above $5 \mathrm{~km}$ (Figure $4.10 \mathrm{C}$ ), and diminishes considerably with depth (Figure 4.10D); we may expect the recovery of shallow $\mathrm{V}_{p}$ structure to have some dependence on shallow $\mathrm{V}_{s}$ anomalies. $\mathrm{A} \mathrm{V}_{p}$-derived volume of point spread functions $\mathrm{V}_{P P}$ shows similar spatial resolution to $\mathrm{V}_{S S}$ (Figure E.3). Amplitudes of $\mathrm{V}_{P P}$ are one order of magnitude smaller than $\mathrm{V}_{S S}$, suggesting that our misfit measurement is more sensitive to heterogeneities in $\mathrm{V}_{s}$ structure. This makes sense to us, as the largest time shifts arise from surface wave measurements which are primarily sensitive to $V_{s}$ structure.

From these results we expect depth resolution to be most strongly dependent on velocity structure in the top $25 \mathrm{~km}$, and consequently we do not interpret velocity changes below $25 \mathrm{~km}$ in this study. Similarly we confine our more targeted interpretations to the central and eastern North Island due to more limited resolution in the western North Island.

\subsubsection{Point-localized perturbations}

To probe the resolution of individual features in our velocity model, we can define the perturbation to be a local point in the model; the computation of point spread functions for all grid points would result in the recovery of the full Hessian (Fichtner and Leeuwen, 2015). Recovery using the action of the Hessian illustrates how the inversion procedure blurs and smears this point-localized perturbation. Due to the discretization of our numerical grid, and the minimum resolvable scale lengths based on the smallest periods in our waveform data, we cannot introduce true point perturbations. Instead our point perturbations are defined by a 3D Gaussian 


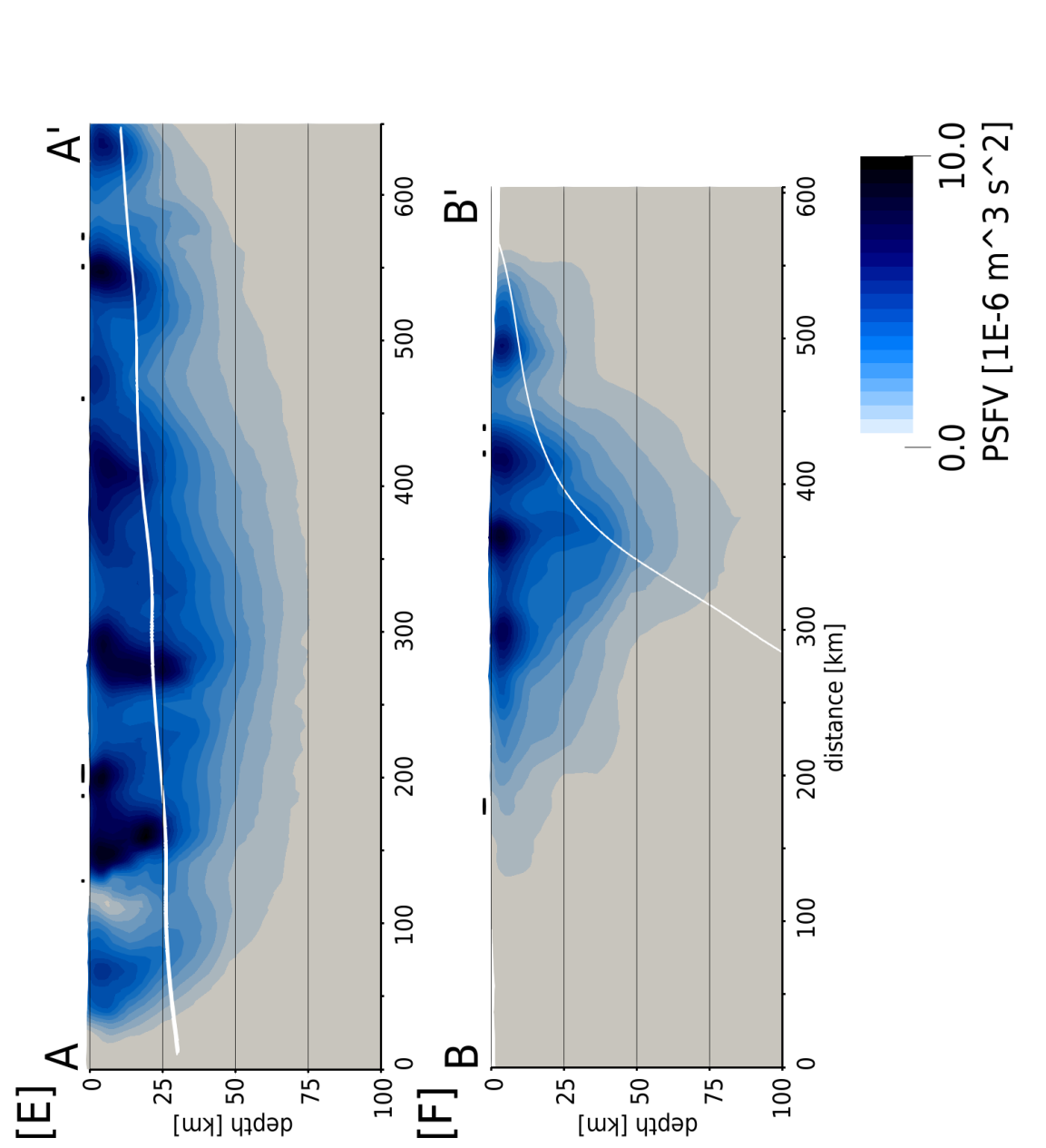

एँ $\dot{D}^{2} \omega^{2}$

उ.

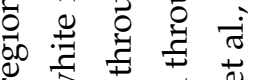

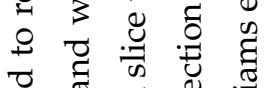

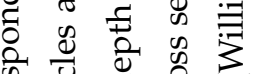

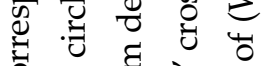

ठ छ

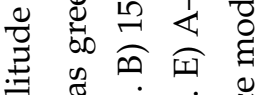

$=$ 学

छ $\sum^{\infty}>^{\infty}$

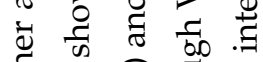

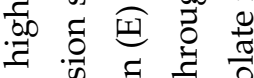

F क्षे

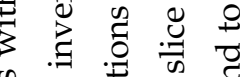

卷.

य

$>$ क 8 ह

$>$ क्षे पै

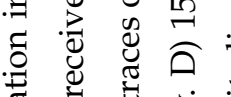

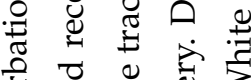

范

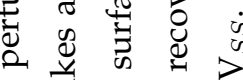

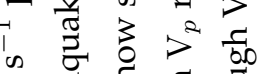
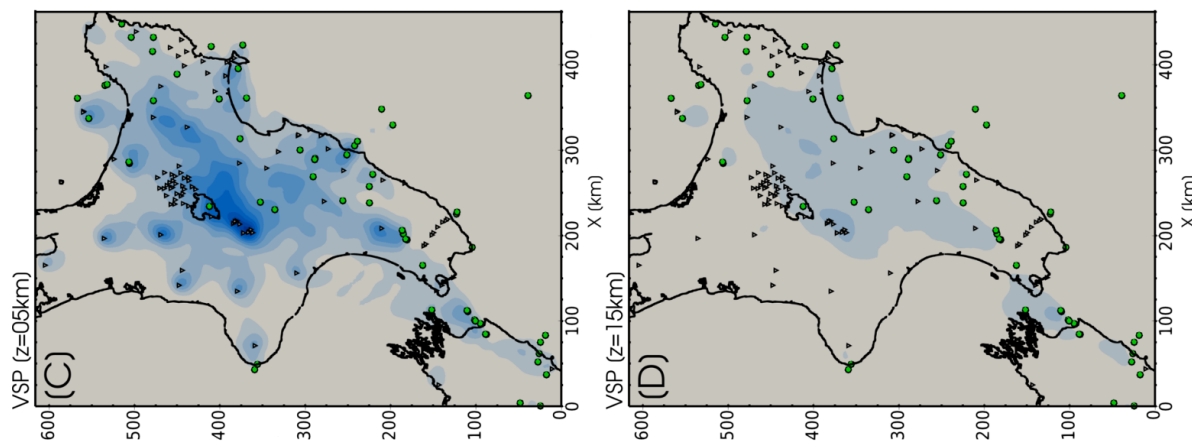

दू क

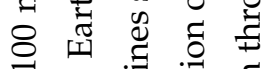

ปิ

\&

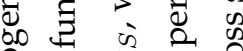

है $>^{\infty}>^{\infty}$

过苟

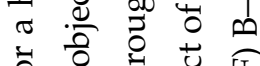

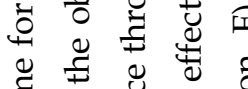

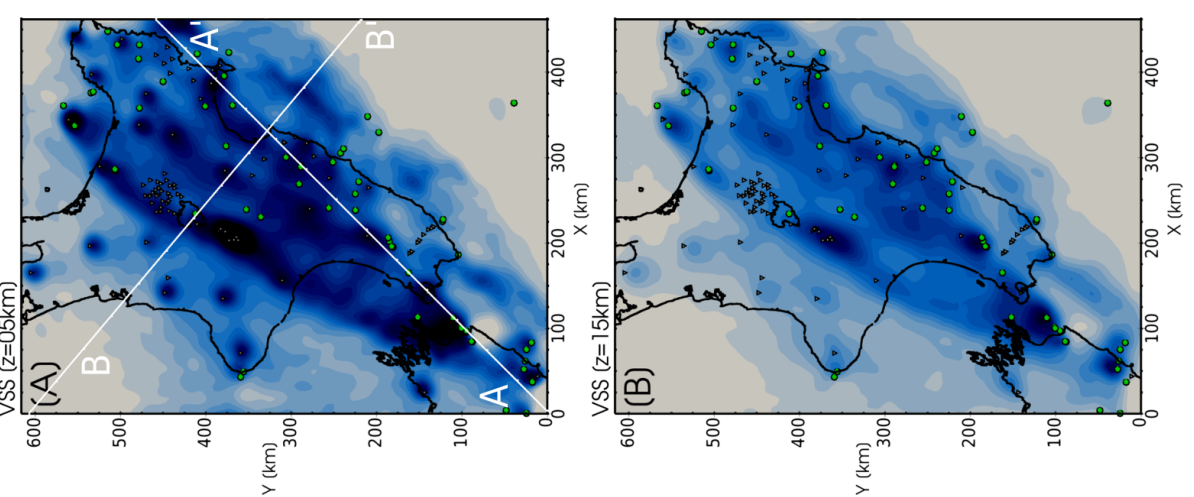

छ

$>$ 品

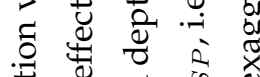

¿ $>^{\infty} 0$

इ

ฮ

के के वे

考

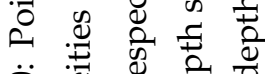

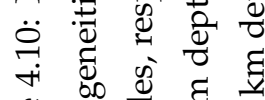

+

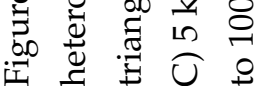


with $\sigma_{h, v}=3 \mathrm{~km}$. Perturbation amplitudes are equal to $\pm 5 \%$ of corresponding $\mathrm{M} 28 \mathrm{~V}_{s}$ model values. We place these point perturbations at various locations around the model, corresponding to the most striking velocity features discussed in Section 4.4 .

The motivation for each point-localized perturbation is to probe the robustness of these features in terms of size, shape, and location. Large-scale lateral smearing, distortion, or non-recovery of perturbations, should indicate poor resolution in corresponding regions. We perform four individual point spread function tests for features A-D labelled in Figure 4.4. The results are discussed in Chapter 5 alongside tectonic interpretations made for each feature.

\subsection{Conclusions}

We perform 28 L-BFGS iterations to improve a starting 3D velocity model of the North Island of New Zealand using spectral element and adjoint methods. Datasynthetic waveforms for 60 events recorded on up to 88 broadband seismic stations are compared in automatically selected time windows, and quantified using a traveltime cross-correlation objective function. Measurements are made on up to 1800 source-receiver pairs, for a final period range of 4-30 s. Total computational cost totalled approximately 500000 CPU-hours over the course of the inversion. Our final velocity model (M28) is defined in updated $\mathrm{V}_{p}$ and $\mathrm{V}_{s} . \mathrm{V}_{s}$ net model updates show large, heterogeneous velocity changes with respect to the initial model, up to $\pm 30 \%$. In general where resolution is available, velocities are slowed down, existing features are sharpened, and previously unseen velocity anomalies are imaged. Resolution analysis using a volume of point spread functions shows that model updates are resolved best in terms of $\mathrm{V}_{s}$ in the upper $30 \mathrm{~km}$, and around the central and eastern North Island. 


\section{Chapter 5}

\section{Tectonic and geologic interpretations of an adjoint tomography velocity model of the North Island of New}

\section{Zealand ${ }^{\mathbb{2}}$}

We interpret an adjoint tomography velocity model in terms of New Zealand tectonics and geology. Seismic velocities show good agreement with known basement terrane features and geologic cross sections. We observe that material properties are heterogeneous along-strike, contrasting previous terrane-based interpretations linking fluid distribution and terrane boundaries. We observe two localized high-velocity anomalies at plate interface depths below the East Coast, and propose they are previously unidentified deeply subducted seamounts. We corroborate our interpretation with other work and discuss the implications of subducted seamounts on slip behavior and geodetic locking along the Hikurangi margin. To the south, a strong velocity gradient is noted in the Cook Strait, supporting

\footnotetext{
${ }^{1}$ As of August 2021, two companion manuscripts based on results presented in this chapter are under peer review with the Journal of Geophysical Research: Solid Earth: (1) Bryant Chow, Yoshihiro Kaneko, Carl Tape, Ryan Modrak, Nick Mortimer, Stephen Bannister, John Townend, Strong upper-plate heterogeneity at the Hikurangi subduction margin (North Island, New Zealand) imaged by adjoint tomography, (2) Bryant Chow, Yoshihiro Kaneko, John Townend, Evidence for deeply-subducted lower-plate seamounts at the Hikurangi subduction margin: implications for seismic and aseismic behavior
} 
hypotheses of a structural boundary here separating the North and South Islands of New Zealand. In the central North Island, velocity anomalies are linked to surface geology, and potential images of crustal magmatic activity below the Taupō Volcanic Zone.

\subsection{Introduction}

Tomographic velocity models can be used to provide direct estimates of material properties within the Earth. Seismic velocities can be related to material properties through the Lamé parameters, which can be used to further constrain other physical quantities such as pressure, temperature, and composition. Seismic velocities can also be interpreted directly as tectonic and geologic features, through comparisons with known and expected regional features (e.g. Tape et al., 2010. Eberhart-Phillips and Reyners, 2012; Eberhart-Phillips and Bannister, 2015). In this chapter, we discuss possible interpretations of the most striking velocity anomalies observed in Chapter 4

The aim of this chapter is three-fold:

1. Identify and interpret the most striking velocity changes in M28;

2. Quantify the robustness of features using point spread functions;

3. Corroborate interpretations with previous studies and discuss implications.

\subsection{Discussion}

\subsubsection{Comparisons with geology and tectonics}

The broad-scale geologic setting of New Zealand is comparable to shallow, laterallyheterogeneous seismic velocities recovered in M28 (Figure 5.1). Absolute wavespeeds are often related to rock type, while ratios of seismic wavespeeds $\left(V_{p} / V_{s}\right)$ can be used to infer the presence of fluids. Similarly, strong, lateral wavespeed contrasts can be attributed to faults or major tectonic boundaries (e.g. Tape et al., 2010; Ellis et al., 2017). Heterogeneous velocity changes in M28 are recovered best in $\mathrm{V}_{s}$ (Figure 4.4 , so throughout this section we primarily discuss $V_{s}$ and $V_{p} / V_{s}$ structure. 


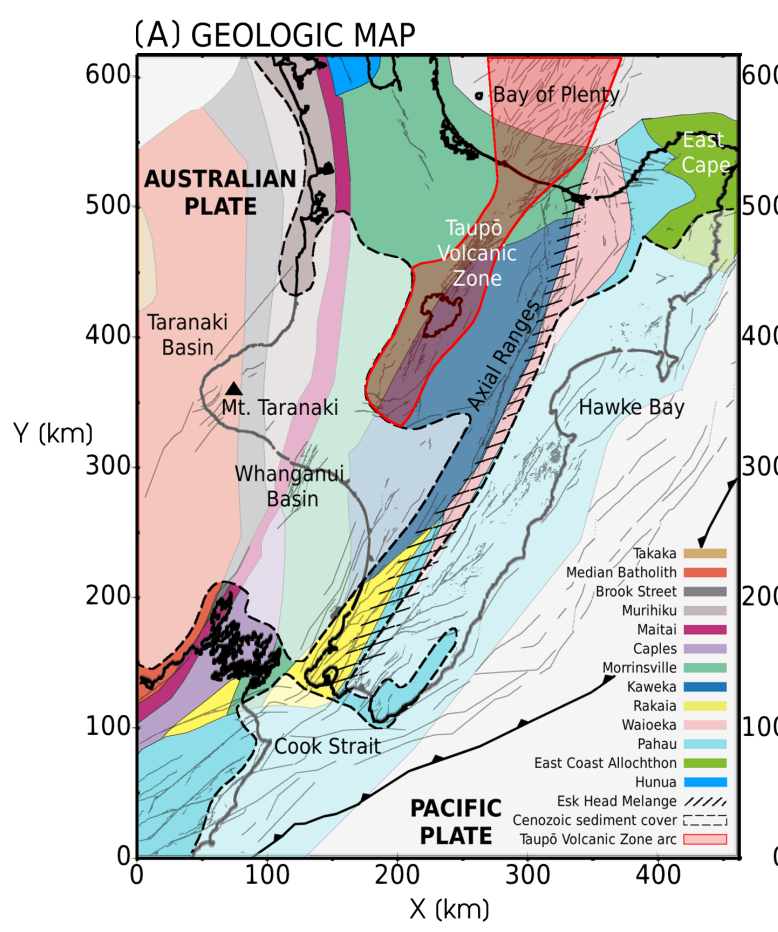

(B) M28VS (z=03km)
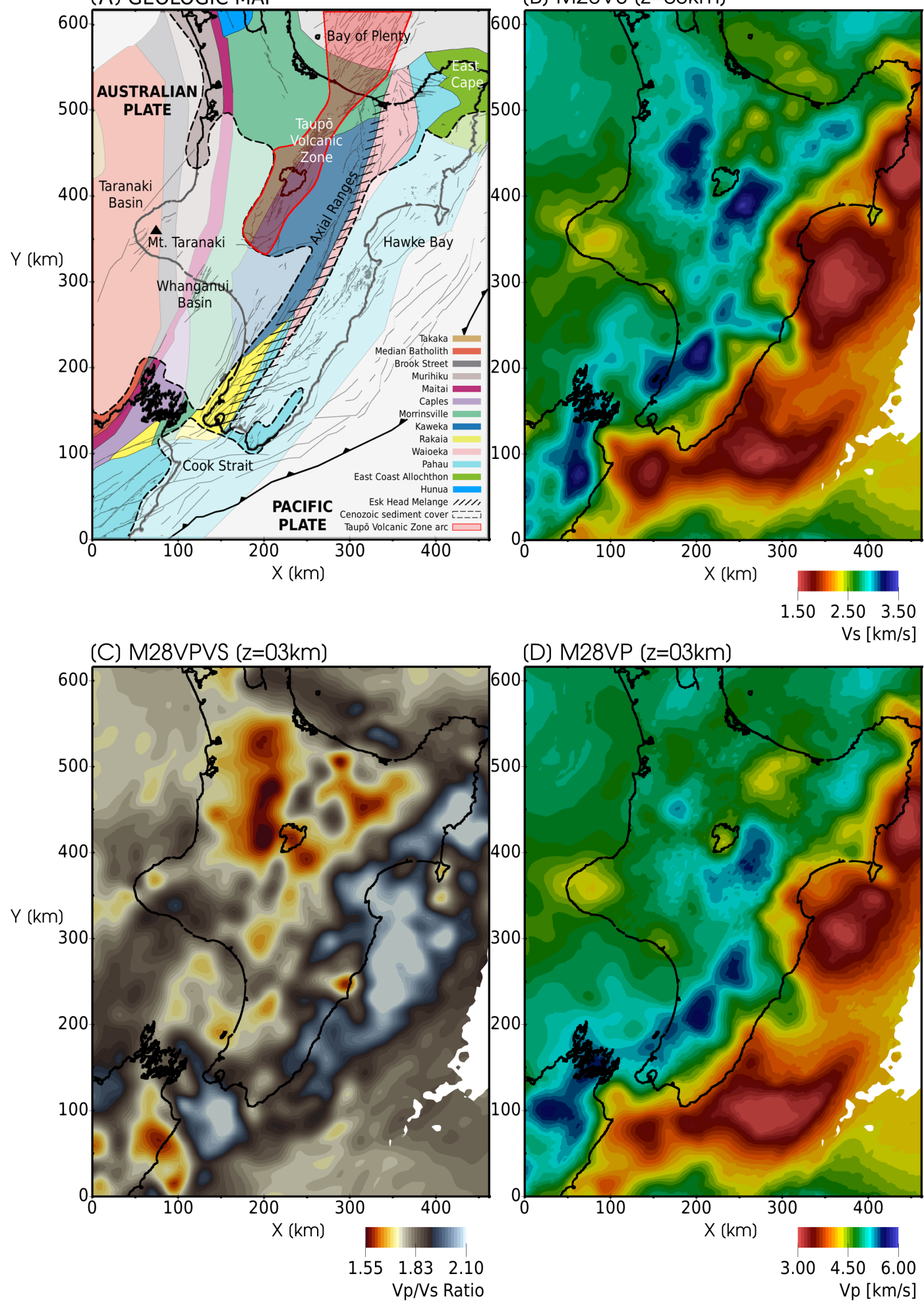

(D) M28VP (z=03km)

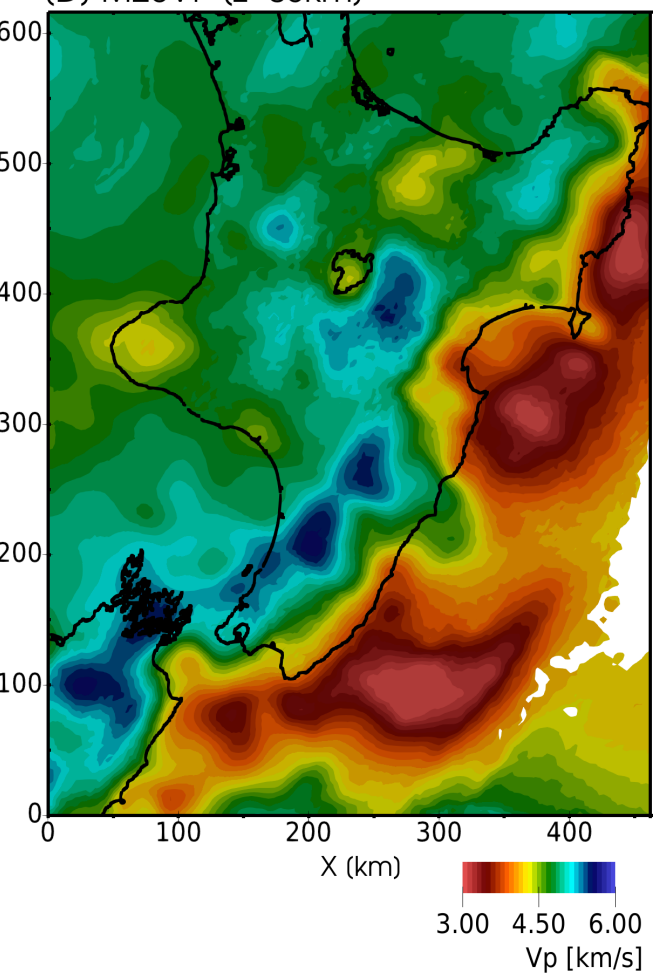

Figure 5.1: A comparison of the M28 velocity model at $3 \mathrm{~km}$ depth, with New Zealand geology. A) New Zealand geology after Mortimer (2004); Edbrooke et al. (2015). Thin gray lines show active faults (Litchfield et al. 2014). B) M28 $\mathrm{V}_{s}$ velocity model at $3 \mathrm{~km}$ depth. C) $\mathrm{M} 28 \mathrm{~V}_{p} / \mathrm{V}_{s}$ at $3 \mathrm{~km}$ depth. D) M28 $\mathrm{V}_{p}$ velocity model at $3 \mathrm{~km}$ depth. White region at the right side of the velocity models corresponds to bathymetry deeper than $3 \mathrm{~km}$, hence no velocity values are available. 
New Zealand geology can be categorized simply as a series of geologically distinct terranes separated by faults or melanges (Mortimer, 2004). A comparison of these terranes (Figure 5.1 A) and the M28 V model (Figure 5.1B) shows broadscale agreement. Shallow $\mathrm{V}_{p} / \mathrm{V}_{s}$ structure follows similar broad-scale trends as $\mathrm{V}_{s}$ (Figure 5.1 C), as does $\mathrm{V}_{p}$ structure, although with some disagreement observed in the northwest North Island (Figure 5.1D). Large-scale (100s km) shallow velocity structure is dominated by the contrast between the exposed basement terranes to the west $\mathrm{V}_{s}>2.5 \mathrm{~km} \mathrm{~s}^{-1}$, and the low-velocity forearc basin, and accretionary wedge offshore to the east $\left(\mathrm{V}_{s}<2 \mathrm{~km} \mathrm{~s}^{-1}\right)$ (Figure $5.1 \mathrm{~B}$ ). The boundary between these two tectonic features lies just inland of the East Coast and spatially correlates with the axial ranges separating the volcanic arc and forearc basins, as well as the Esk Head Melange separating the Kaweka and Pahau/Waioeka terranes (white stippled line, Figure 5.1A). In $\mathrm{V}_{p} / \mathrm{V}_{s}$ this boundary is less well defined but spatially similar, as the juxtaposition of high $\mathrm{V}_{p} / \mathrm{V}_{s}(>2)$ in the forearc region against lower $\mathrm{V}_{p} / \mathrm{V}_{s}(<1.8)$ to the west, which could be the boundary between lower-velocity, fluid-saturated forearc sediments, and exposed basement rocks of the upper plate (Figure 5.1C). Visible in both $\mathrm{V}_{s}$ and $\mathrm{V}_{p} / \mathrm{V}_{s}$ along this boundary are shallow expressions of the two high-velocity, low $\mathrm{V}_{p} / \mathrm{V}_{s}$, anomalies identified as features A and B in Chapter 4 , which will be discussed in detail in Section 5.2.3. Moderate-sized velocity features $(\sim 50 \mathrm{~km})$ correspond well to basement terranes and individual tectonic features around the North Island. High $V_{s}$ (Figure 5.1 B) and moderate to low $\mathrm{V}_{p} / \mathrm{V}_{s}$ (Figure 5.1C) below the central North Island $\left(\mathrm{V}_{s}>3 \mathrm{~km} \mathrm{~s}^{-1}\right.$; $\mathrm{V}_{p} / \mathrm{V}_{s}<1.8 \mathrm{~km} \mathrm{~s}^{-1}$ ) striking northeast-southwest show good agreement with the Waioeka, Kaweka, and Rakaia terranes (Figure 5.1A). In the northeastern corner of the domain, near East Cape, a notch of high velocities $\left(\mathrm{V}_{s}>2.5\right.$; Figure 5.1B) shows similar shape to the boundary between the Pahau terrane and the East Coast Allocthon, a body of tectonically displaced sedimentary and volcanic rock. West of the TVZ, high velocities $\left(\mathrm{V}_{s}>3.5 \mathrm{~km} \mathrm{~s}^{-1}\right)$ extends northward, spatially correlating with the western boundary of the Morrinsville terrane (Figure 5.1A). Corresponding high velocities are not seen in $\mathrm{V}_{p}$ (Figure 5.1D), potentially due to the lack of $\mathrm{V}_{p}$ resolution in this region. With sparse seismicity here, shallow surface waves recorded at western North Island stations may have enhanced the relative sensitivity of $\mathrm{V}_{s}$ structure, consequently leading to the low $\mathrm{V}_{p} / \mathrm{V}_{s}(<1.6)$ values seen here (Figure 5.1 C). In the northern South Island, the Caples terrane, overprinted 
by high-velocity schist, has previously been noted as a distinct patch of high $\mathrm{V}_{p}$ by Eberhart-Phillips et al. (2005). The northward extent of high $\mathrm{V}_{p}$ in M28 (Figure 5.1D) stops at the landward extent of the South Island, however improved offshore resolution in $\mathrm{V}_{s}$ shows a patch of high velocity $\left(\mathrm{V}_{s} \approx 3 \mathrm{~km} \mathrm{~s}^{-1}\right)$ extending offshore, generally coinciding with the offshore extent of the Caples terrane (Figure 5.1B)

Other geologic features can be seen to overly the basement terranes of the North and South Islands. In the Taupō volcanic zone, low velocity $\left(\mathrm{V}_{s}<2.5 \mathrm{~km} \mathrm{~s}^{-1}\right)$ and high $\mathrm{V}_{p} / \mathrm{V}_{s}(>1.8)$ extending from Ruapehu northwest into the Bay of Plenty (Figure $5.1 \mathrm{~B}, \mathrm{C}$ ) is likely related to magmatic processes in the active volcanic arc. Distinct low-velocity anomalies $\left(\mathrm{V}_{s} \sim 2 \mathrm{~km} \mathrm{~s}^{-1}\right)$, tens of $\mathrm{km}$ wide, make up the lowvelocity zone seen in the TVZ, and are not visible in $V_{p}$ (Figure 5.1D), resulting in resolution of high $\mathrm{V}_{p} / \mathrm{V}_{s}(>1.8)$ egg-shaped anomalies (Figure 5.1C). We discuss the TVZ in further detail in Section 5.2.5 (Figure 5.1B, C). Patches of low velocity (both $\mathrm{V}_{p}$ and $\mathrm{V}_{s}$ ) are seen along the west coast of the North Island, and are most likely associated with the ring plain of Taranaki Volcano, and low-velocity sediments in the Taranaki and Whanganui Basins (Figure 5.1A). In the southern end of the domain, strong velocity gradients are observed separating high velocity $\left(\mathrm{V}_{s}>3 \mathrm{~km} \mathrm{~s}^{-1}\right)$ of the North and South Islands, with low velocities $\left(\mathrm{V}_{s}<2 \mathrm{~km} \mathrm{~s}^{-1}\right)$ in the Cook Strait. This is discussed in more detail in Section 5.2.4, Offshore the East Coast, the low-velocity accretionary wedge dominates the landscape with average $\mathrm{V}_{s}$ values between 1 and $2 \mathrm{~km} \mathrm{~s}^{-1}$. The far offshore corners of the domain northwest of Taranaki and east of the Hikurangi trench, have no resolution and consequently show little detail in velocity structure.

We compare our M28 $\mathrm{V}_{s}$ model to two geologic cross sections along the East Coast (Figure 5.2). These geologic cross sections were derived from seismic lines and surface geology, and constrained using oil exploration well logs (Francis et al., 2004). In the two examples, crossing through Northern Hawke's Bay (Figure 5.2 C, E), and Central Hawke's Bay (Figure 5.2 D, F), the M28 V model shows good agreement with inferred shallow structure. Basin-like sedimentary features ranging from the surface down to $\sim 10 \mathrm{~km}$ depth can be observed in both Northern and Central Hawke's bay, providing a direct link between $\mathrm{V}_{s}$ velocities and direct geologic observations of crustal structure, deposition age and sedimentary composition. In these two cross-sections, velocities ranging between $1.5 \leq \mathrm{V}_{s} \leq 2.2 \mathrm{~km} \mathrm{~s}^{-1}$ relate 


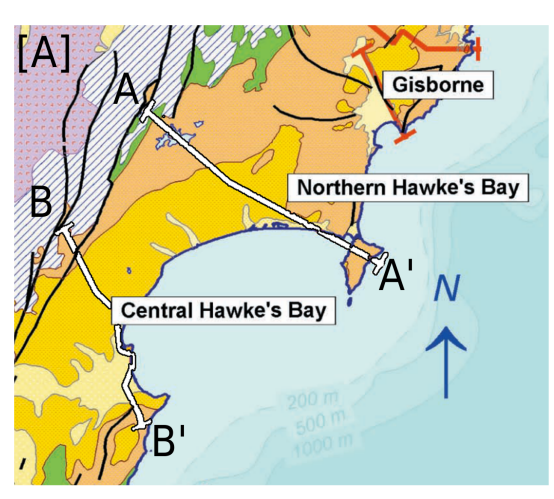

[C] Northern Hawke's Bay
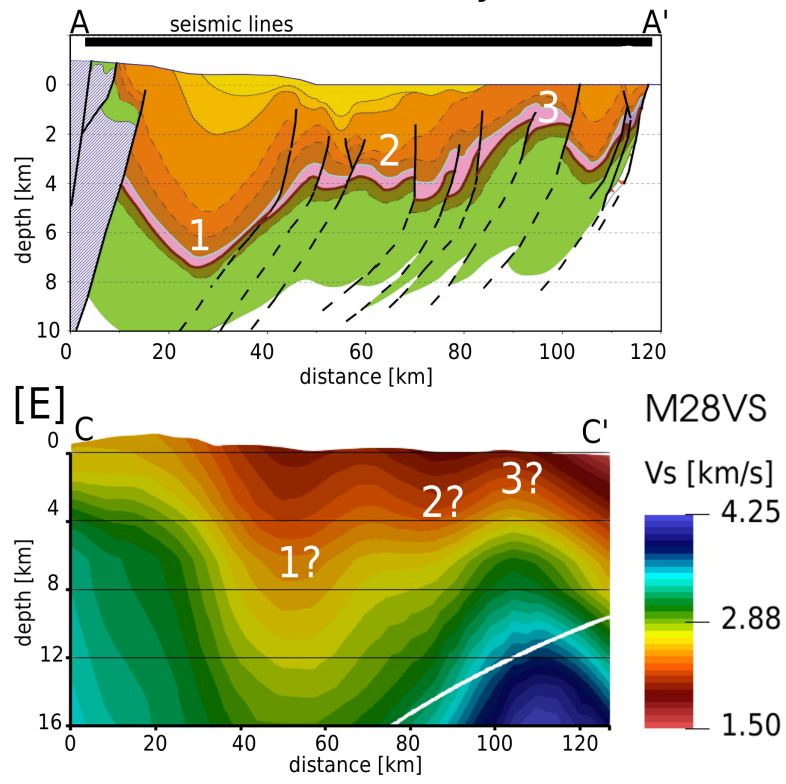
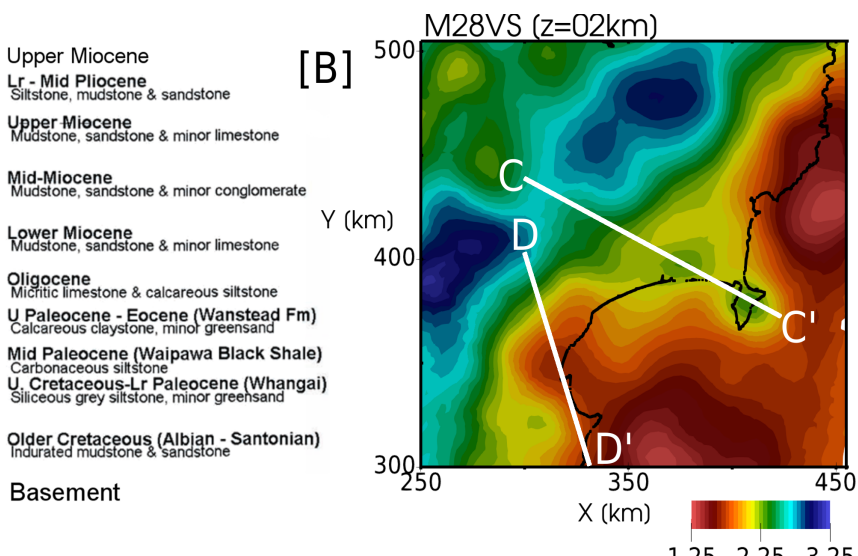

[D] Central Hawke's Bay $v_{s}[\mathrm{~km} / \mathrm{s}]$

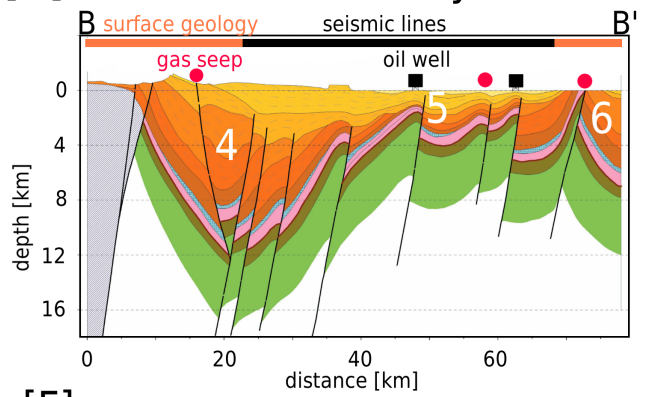

[F]

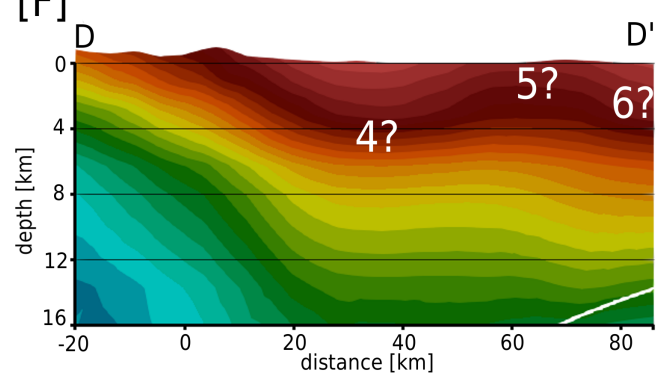

Figure 5.2: A comparison of geologic cross sections with the $M 28 \mathrm{~V}_{s}$ velocity model. A) Map view of the Hawke's Bay region, adapted from Figure 6 of Francis et al. (2004). Colors correspond to sedimentary rock types in legend. A- $\mathrm{A}^{\prime}$ and $\mathrm{B}-\mathrm{B}^{\prime}$ shows surface trace of cross section shown in (C) and (D). B) $2 \mathrm{~km}$ depth slice of M28 $\mathrm{V}_{s}$. White line $\mathrm{C}-\mathrm{C}^{\prime}$

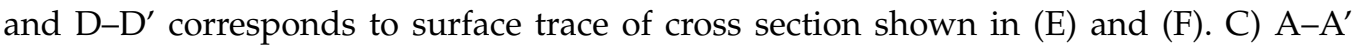
geologic cross section through Northern Hawke's Bay, adapted from Figure 9 of Francis et al. (2004), at 5x vertical exaggeration. Cross-section derived using seismic lines. Features 1, 2, and 3 used for comparisons with velocity model shown in (E). Black lines represent faults, with dashed lines referring to inferred fault continuation. D) B-B' Central Hawke's Bay geologic cross section at $2 \mathrm{X}$ vertical exaggeration, derived from surface geology and seismic lines, adapted from Figure 10 of Francis et al. (2004). Gas seeps and oil well locations shown as red circles and black squares, respectively. Features 4, 5, and 6 correspond to features in (F). E) C- $\mathrm{C}^{\prime}$ cross section through M28 $\mathrm{V}_{s}$ model at $5 \mathrm{x}$ vertical exaggeration. White solid line shows plate interface model of Williams et al. (2013). Corresponding velocity features 1,2 , and 3 from (C). F) D-D' cross section through M28 $\mathrm{V}_{s}$ model at $3 x$ vertical exaggeration. Corresponding velocity features 4,5 , and 6 from (D). 
to Miocene-age sedimentary cover, while high velocities $\left(\mathrm{V}_{s}>3.5 \mathrm{~km} \mathrm{~s}^{-1}\right)$ generally coincide with basement rocks. Velocity layers here correspond well to the composition-related delineations made on the geologic cross sections at vertical resolutions of $\sim 2 \mathrm{~km}$, and horizontal resolutions of about $\sim 20 \mathrm{~km}$, which as an indication of good shallow resolution in our $\mathrm{V}_{s}$ model.

\subsubsection{Along-strike crustal heterogeneity}

Material heterogeneity along-strike of the Hikurangi margin has been proposed as a potential explanation for the observed locked-to-creeping transition zone of the plate interface (Reyners et al., 2017). Figure 5.3 shows comparisons of the $\mathrm{V}_{p} / \mathrm{V}_{s}$ ratio for trench and strike-parallel cross-sections, characterizing along-strike seismic properties of the upper plate and plate interface region. Figure 5.3A shows a strike-parallel cross section through the $\mathrm{M} 00 \mathrm{~V}_{p} / \mathrm{V}_{s}$ model, which is identical to the NZ-Wide2.2 $\mathrm{V}_{p} / \mathrm{V}_{s}$ model of Eberhart-Phillips et al. (2020b). This model was interpreted by Reyners et al. (2017) as showing significantly higher $\mathrm{V}_{p} / \mathrm{V}_{s}(>1.8$ ) to the north where the slip rate deficit is low (blue interface line in Figure 5.3 A). They characterize lower $\mathrm{V}_{p} / \mathrm{V}_{s}(<1.7)$ southward as an increasingly dehydrated upper plate in the southward transition from subduction to continental collision (red interface line in Figure 5.3A). Using relocated seismicity they propose that along-strike fluid distributions arise from variations in permeability of the overlying basement terranes (Mortimer, 2004), postulating that the Rakaia terrane (solid yellow lines in Figure 5.3 A, B, D, E) acts as a permeability barrier in the south, surrounded by a more permeable Pahau terrane (Figure 5.1A).

In contrast to the findings of Reyners et al. (2017), our M28 $\mathrm{V}_{p} / \mathrm{V}_{s}$ model does not show the same linear southward transition from high to low $V_{p} / V_{s}$ along-strike (Figure 5.3 B). Rather, we observe strong heterogeneity in $\mathrm{V}_{p} / \mathrm{V}_{s}$, with alternating patches of high and low $\mathrm{V}_{p} / \mathrm{V}_{s}$ along-strike. If terrane boundaries are the cause of fluid distribution in the upper plate, then we may have expected to see these patches correlate spatially to terrane transitions. The yellow line on Figure $5.3 \mathrm{~B}$ denotes the approximate location of the Rakaia terrane, surrounded by the Pahau terrane (Figure 5.1A), showing little to no correlation of the terrane boundary with corresponding $\mathrm{V}_{p} / \mathrm{V}_{s}$ values. Similarly strike-parallel cross sections through the Rakaia terrane show varying values of $\mathrm{V}_{p} / \mathrm{V}_{s}$, with no strong spatial correlations between $V_{p} / V_{s}$ and the approximate location of the Rakaia terrane (Figure 5.3 D, 

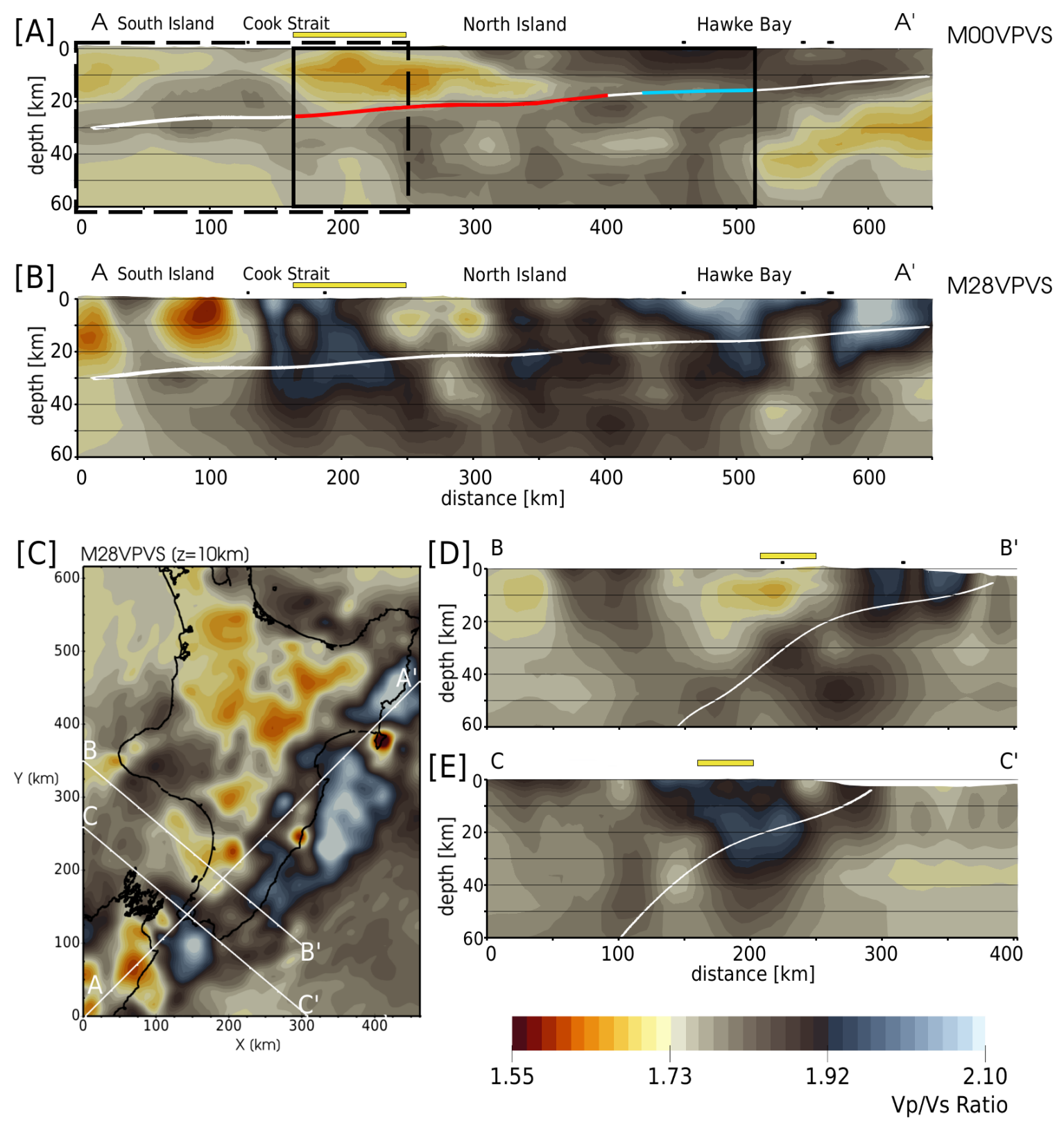

Figure 5.3: Along-strike heterogeneity of $\mathrm{V}_{p} / \mathrm{V}_{s}$. Surface traces of cross sections shown in (C). Cross sections shown to $60 \mathrm{~km}$ depth with $2 x$ vertical exaggeration; solid white line correspond to plate interface model of Williams et al. (2013); solid yellow line shows approximate location of Rakaia terrane (Figure 5.1A). A) A-A' cross section through M00 $\mathrm{V}_{p} / \mathrm{V}_{s}$ model. Solid and dashed black outlines correspond to approximate bounds of Figure 5 (solid) and Figure 6 (dashed) of Reyners et al. (2017). Approximate geographic locations annotated above. Red colors on interface denote where slip rate deficit is $>20 \mathrm{~mm} \mathrm{yr}^{-1}$, while blue colors represent where slip rate deficit is $<10 \mathrm{~mm} \mathrm{yr}^{-1}$,Wallace et al., 2012a; Reyners et al., 2017). B) A-A' cross section through M28 $\mathrm{V}_{p} / \mathrm{V}_{s}$ model. Black dots represent approximate coastline locations, used to demarcate geographic boundaries. C) $10 \mathrm{~km}$ depth slice through M28 $\mathrm{V}_{p} / \mathrm{V}_{s}$ model. Surface traces for corresponding cross sections shown as white lines. D) B-B' cross section through M28 $\mathrm{V}_{p} / \mathrm{V}_{s}$ model. E) $\mathrm{C}-\mathrm{C}^{\prime}$ cross section through $\mathrm{M} 28 \mathrm{~V}_{p} / \mathrm{V}_{s}$ model. 
E).

The M28 velocity model suggests that $\mathrm{V}_{p} / \mathrm{V}_{s}$ structure is correlated more to the interplay of a low-velocity, saturated forearc region, contrasting exposed basement terranes; $\mathrm{V}_{p} / \mathrm{V}_{s}$ reaches maximum values $(>2)$ below the Cook Strait and Hawke Bay, while remaining moderate to low $(<1.7)$ below land (Figure 5.3B). One potential explanation for the large observed discrepancies in updated $V_{p} / V_{s}$ structure may be increased sensitivity to shallow $\mathrm{V}_{s}$ structure through the inclusion of surface wave measurements, leading to improved crustal, short-wavelength resolution of $\mathrm{V}_{p} / \mathrm{V}_{s}$. If the initial model of Eberhart-Phillips et al. (2020b) is only sensitive to the very long-wavelength $(>50 \mathrm{~km})$ fluid distribution imaged by $\mathrm{V}_{p} / \mathrm{V}_{s}$, then lateral smearing of deeper structure may have influenced values at mid-crustal depths. We do observe that terrane boundaries can exert controls on upper plate heterogeneity through velocity structure, however we believe that the direct explanation of slip rate deficit by terrane boundaries may require re-interpretation with this updated velocity model, and that permeability control by upper plate composition is insufficient to explain the heterogeneous $V_{p} / V_{s}$ structure we observe.

We note that one point of concern in interpreting our $\mathrm{V}_{p} / \mathrm{V}_{s}$ model, derived from direct division of $V_{p}$ by $V_{s}$, is the possibility of apparent $V_{p} / V_{s}$ structures arising from differences in resolution of $\mathrm{V}_{p}$ and $\mathrm{V}_{s}$ within our inversion. The main cross section (Figure 5.1B) crosses through a region that is well sampled by our dataset (Figure 4.1), providing confidence that we have resolution in both $\mathrm{V}_{p}$ and $\mathrm{V}_{s}$ here, however further resolution analysis is required to quantitatively distinguish resolved $\mathrm{V}_{p} / \mathrm{V}_{s}$ structures from those that may arise from resolution differences.

\subsubsection{East Coast high-velocity anomalies}

We propose that the high-velocity anomalies below the east coast of the North Island are two previously unidentified subducted seamounts (Figure 5.4). At $12 \mathrm{~km}$ depth, where the subduction interface is roughly below the coastline (Figure 5.4A), two high-velocity, point-like features are visible below Māhia Peninsula (labelled $M$ in Figure 5.4A-D) in the north, and Pōrangahau (labelled P in Figure 5.4A-D) to the south. Cross-sections show a section of relatively high velocities extending above and below the predicted interface (Figure 5.4B-D). The velocity anomaly be- 
low Pōrangahau features a corresponding low-velocity zone further seaward that is not seen at Māhia Peninsula.

A point spread test was performed to understand the robustness of these features, and the overall distortion accompanying recovery of a point perturbation in these locations (Figure 5.4E-L). Positive velocity input perturbations were placed around interface depths below Māhia Peninsula (Figure 5.4E, G) and Pōrangahau (Figure 5.4[, K). The resulting point spread functions show acceptable recovery of input locations, but with considerable lateral smearing (Figure 5.4F, J, H, L). In depth slice (Figure 5.4F, J), lateral smearing along the low-velocity forearc region can be seen, most likely owing to the predominant use of surface waves which are laterally not well constrained in the near offshore region. In cross-section (Figure $5.4 \mathrm{H}, \mathrm{L}$ ), both features are smeared above and below the perturbation, from below the interface to the surface, and slightly landward in the horizontal direction, causing recovery of the perturbations to appear taller and wider than the input. Despite the distortion, recovery of these features is possible, but the inversion lacks vertical constraint, and consequently the high velocities seen above and below these velocity anomalies may not be physical.

We interpret these two high-velocity anomalies as previously unidentified subducted seamounts, given their depths, point-like shape, and corroboration with external evidence (Figure 5.5). To first order the velocity signatures cover a footprint on the order of tens of $\mathrm{km}$. The subducting seafloor offshore the east coast of the North Island is dotted with seamounts at various stages of subduction and under varying levels of sediment cover (Wood and Davy, 1994; Davy and Wood, 1994; Davy et al., 2008). Marine seismic reflection has provided images of seamounts below the accretionary wedge, for example correlated to low reflectively zones and positive magnetic anomalies offshore Māhia Peninsula and Gisborne (Bell et al., 2010), as well as further trenchward, linked with bathymetric features like Rock Garden and Bennett Knoll (Figure 5.5 C; Barnes et al., 2010). These offshore seamounts have similar estimated footprints on the scale of tens of $\mathrm{km}$, and heights of a few km (Barnes et al., 2010; Bell et al., 2010).

Offshore Māhia Peninsula, the Poverty Re-entrant is a large double collapse feature previously interpreted as a seamount scar that may have resulted from two consecutive seamount impacts over the last 1-2 Myr (Figure 5.5C; Lewis and Pet- 

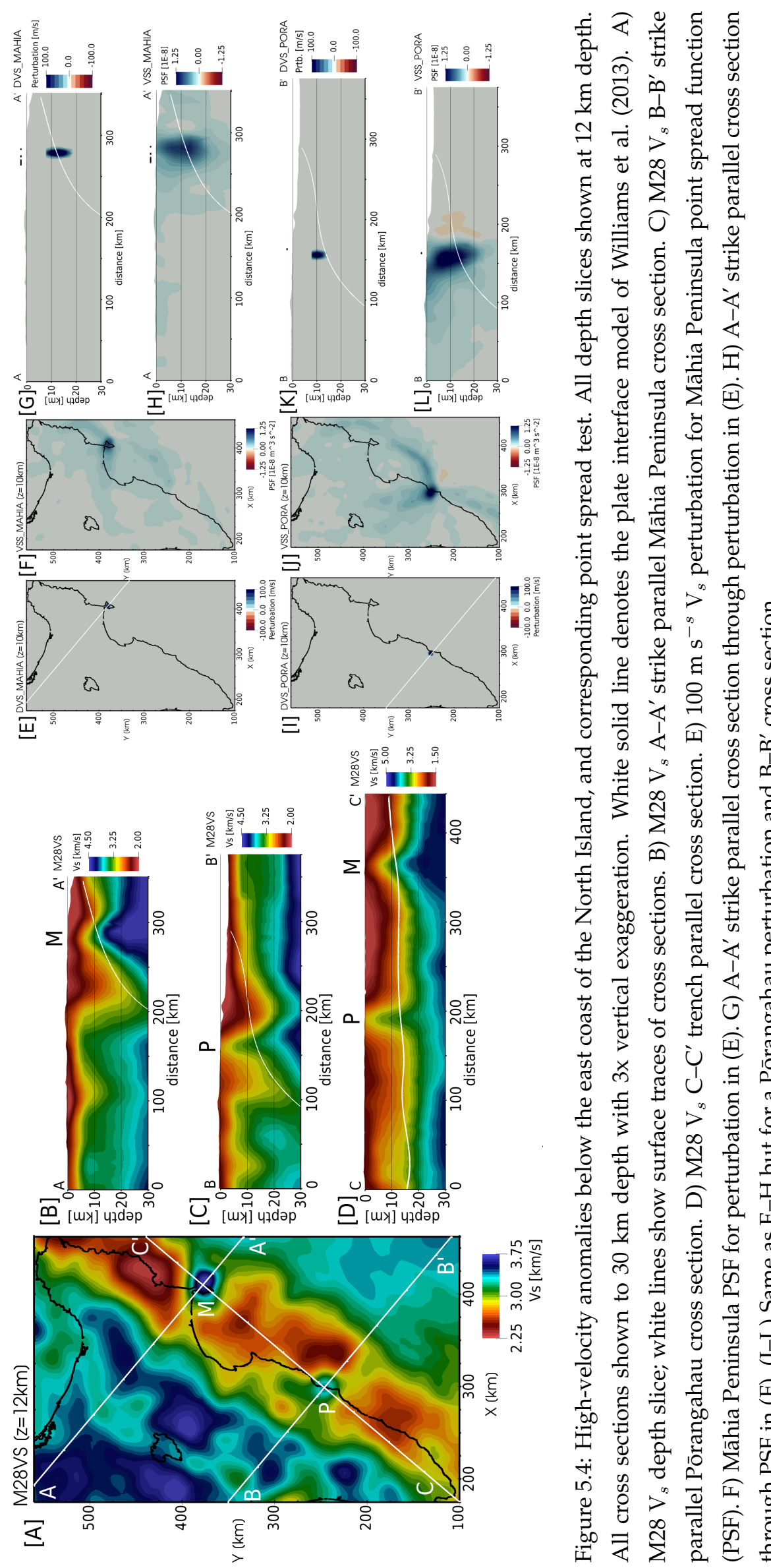

चี

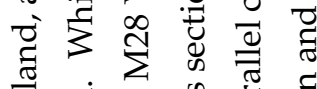
ज

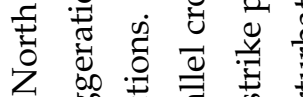

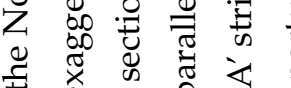
† 盛

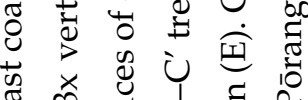
ฮै ले छ

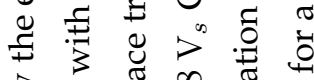

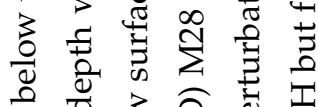
ช 曾

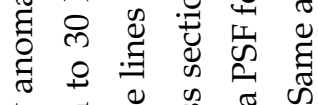

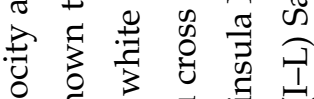

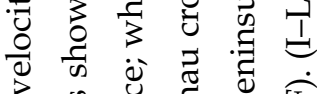
㲾

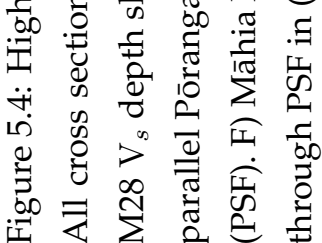


tinga, 1993; Collot et al., 1996; Lewis et al., 1998; Pedley et al., 2010). Further South, the prominent Madden Canyon sits just offshore Pōrangahau (Figure 5.5C). These features can be linked to seamount subduction through sandbox experiments, which have shown a variety of distinct, identifiable, features related to seamount subduction, including: a re-entrant in the frontal wedge, local uplift above the seamount controlled by size and shape, and subsidence in the wake of the seamount leading to mass-sliding and canyon erosion (Figure 5.5B; Dominguez et al., 1998, 2000).

At the Hikurangi subduction zone, we would expect features inferred by sandbox experiments to strike parallel to the plate convergence direction, putting Poverty Re-entrant and Madden Canyon in line with these seamounts. We propose that the collision of the imaged seamount below Māhia Peninsula with the frontal wedge is the cause of the lower, V-shaped Poverty re-entrant (solid green line, Figure 5.5 C). The wider, upper Poverty indentation (stippled green line, Figure 5.5C) may be related to local subsidence in the trailing flank of the seamount, or to another, more deeply subducted, seamount that is not resolvable in our model. Roughly $10 \mathrm{~km}$ off the coast of Māhia Peninsula, the Lachlan fault system dips landwards as a listric thrust complex that has seen almost $6 \mathrm{~km}$ vertical separation of its northern segment offshore Māhia Peninsula, with respect to its southern extent, hypothesized as upper plate response to a subducted seamount $>10 \mathrm{~km}$ below the Peninsula (Barnes et al. 2002). Seamount-derived uplift may be the origin of the anomalous, sub-aerial, topographic high of Māhia Peninsula. Similar to Poverty indentation, we propose Madden Canyon may have developed as the result of localized subsidence in the trailing flank of the seamount below Pōrangahau, which created a preference for mass transport through this area, and subsequent canyon erosion. There is no obvious re-entrant for the Pōrangahau seamount, which may be explained by the relatively shallow taper of the accretionary wedge compared to Māhia Peninsula; any corresponding re-entrant feature would be far offshore and may have become obscured by further subduction, erosion, trench fill, or other seamount impacts.

Further evidence points to anomalous geophysical behavior at these two locations. The locked-to-creeping transition zone occurs near Pōrangahau, with the southern extent of shallow slow slip patches on the Hikurangi mega-thrust terminating here; the highest slip patches of these shallow SSEs anomalously show segmen- 


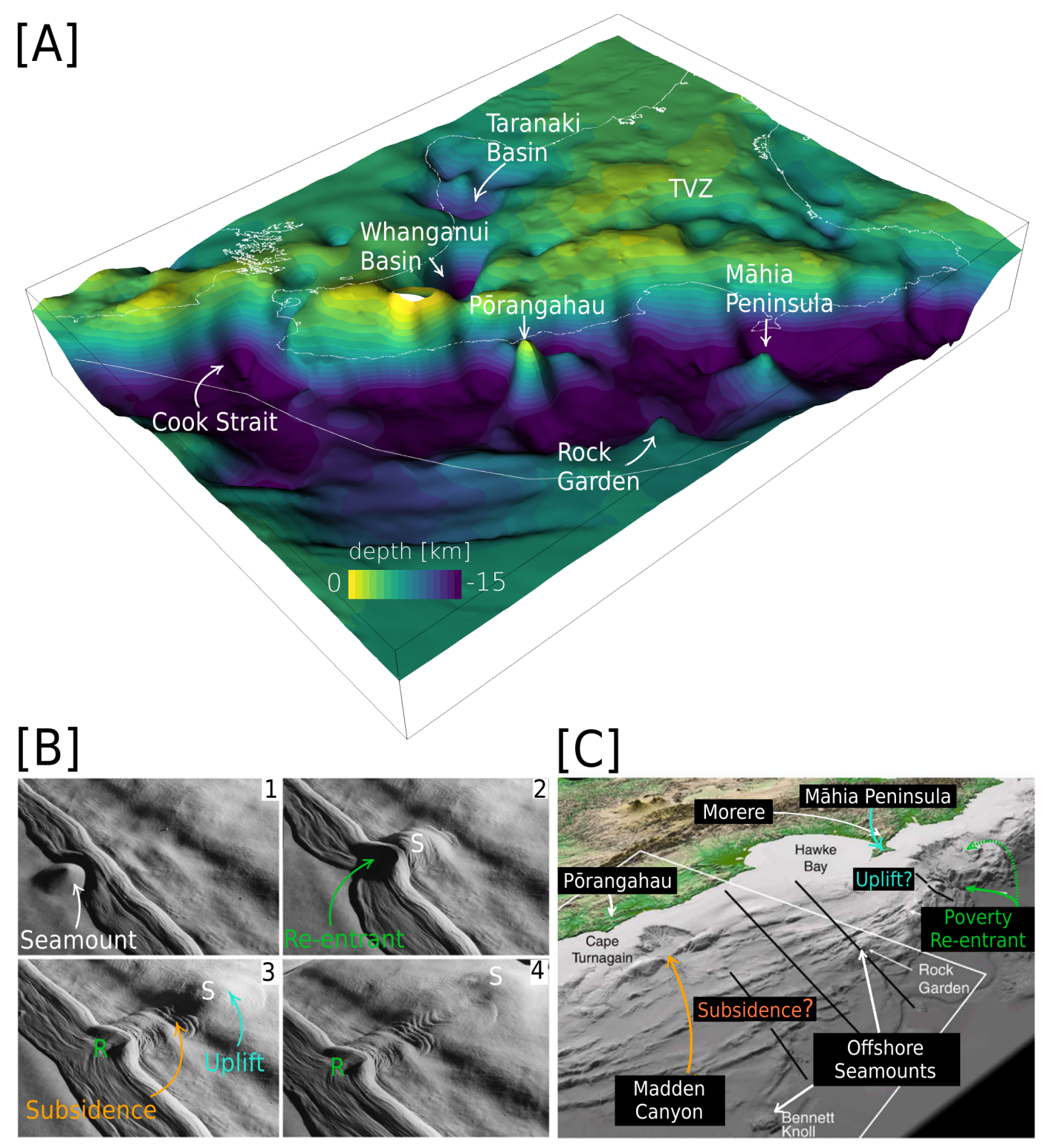

Figure 5.5: Evidence for deeply subducted seamounts. A) $\mathrm{V}_{s}=3 \mathrm{~km} \mathrm{~s}^{-1}$ isocontour through the M28 velocity model, colored by corresponding depth values, looking to the northwest, or strike parallel. Thin white lines correspond to coastline and trench. Distinct point-like high-velocity anomalies visible below Pōrangahau and Māhia Peninsula, interpreted as subducted seamounts (Section 5.2.3). Also visible are strong velocity gradients associated with the Cook Strait, Whanganui basin, and Taranaki basin (Figure 5.1). B) Sand table seamount subduction experiments modified from Dominguez et al. (1998), showing upper plate deformation caused by a subducting conical seamount. The most striking results are the re-entrant collapse feature left in the accretionary front, localized uplift on the leading flank, and localized subsidence above the trailing flank. C) Offshore bathymetry of the East Coast adapted from Barnes et al. (2010). Offshore seamounts have been detected in various stages of subduction into the frontal wedge. Two significant bathymetric features directly offshore the proposed locations of subducted seamounts are Poverty re-entrant and Madden canyon. 
tation into two distinct patches roughly between Cape Turnagain, Cape Kidnappers, and Gisborne (Figure 1.3. Wallace, 2020). Additionally, repeated episodes of swarm seismicity have been observed near Pōrangahau (Jacobs et al., 2016). Approximately $20 \mathrm{~km}$ landward of Māhia Peninsula, the Morere thermal spring is one of only two thermal springs in this region (Reyes et al., 2010), and coincides with the Morere magnetic anomaly (+70 nT; Hunt and Glover, 1995), as well as an $8 \mathrm{~km}$ high $\mathrm{V}_{p}$ anomaly thought to be of volcanic origin (Figure 5.5, Eberhart-Phillips et al. 2015). Magnetotelluric studies here show a resistive patch on the plate interface below Māhia peninsula, with a more conductive patch further landward coinciding with the Morere anomaly, and inferred as fluid- or clay-rich sediments (Heise et al. 2017). Bell et al. (2010) propose that high reflectively zones seen at the leading flanks of offshore seamounts are underthrusted, fluid-rich sediments; if correct, then deeply subducted, fluid-rich sediments at the leading flank of the seamount below Māhia Peninsula may be the source of the various Morere anomalies.

The effect of seamount subduction on interface geometry, seismicity, and fluid distribution is a topic of current study. For example Sun et al. (2020) performed finiteelement modelling of 2D seamount subduction and their model predicted enhanced consolidation and tectonic compression in the leading flank of a seamount, and a preference for storage of elastic strain and seismicity. In the trailing flank, their model predicts a stress shadow, with reduced tectonic compression, upperplate faulting and damage, and a preference for aseismic slip.

We propose that the seamounts below Pōrangahau and Māhia Peninsula have left a trail of inelastically deformed and fractured upper plate in their wake, since initial impact at the trench. As suggested by Cummins et al. (2002) for the Nankai Trough, this would prevent elastic strain accumulation necessary for seismic rupture propagation. Anomalously shallow SSEs observed in the northern Hikurangi margin (Figure 1.3), as well as the segmentation of the slow slip patches, may reflect the first order path of the two seamounts, and the relatively undamaged upper plate between, which has retained its mechanical integrity. Tectonic compression in the leading edge of the seamounts may also coincide with the anomalously high seismicity observed at Pōrangahau. Differences in observed behavior between the two seamounts might be attributed to differences in size, shape, aspect ratio, and subducted depth, all of which will have an effect on the upper-plate 
response (Dominguez et al., 1998, 2000; Sun et al., 2020).

The relationships between subducted seamounts and entrained sediments, their interplay with fluid content and slip behavior, and the consequential effects on gravitational and magnetic anomalies, long-wavelength topographic features, coastal uplift and marine terraces, or surface seeps and thermal springs, remains unclear. More targeted studies will be required to determine the relationships between these inferred seamounts and other relevant observations in these areas.

\subsubsection{Cook Strait velocity gradient}

A strong velocity gradient in the Cook Strait is observed in the upper $10-15 \mathrm{~km}$, resulting in steep relief to the west against the South Island, and to the north (labels $\mathrm{W}$ and $\mathrm{N}$ respectively, in Figure 5.6). Shallow $(<5 \mathrm{~km})$ velocity structure offshore should be dominated by low-velocity sedimentary basins. Uruski (1992) identified three, thick, sedimentary basins in the Cook Strait: the Wairau, Wairarapa, and Flaxbourne (Clarence) basins, characterized by negative gravity anomalies and significant depth to basement. From seismic lines, significant two way travel times (3-4 s TWT) hinted at $>3 \mathrm{~km}$ deep sediment fills, while imaged basin geometries suggested that they developed as pull-apart basins in a strike-slip regime (Uruski, 1992; Barnes and Audru, 1999). Dashed white lines in Figure 5.6A show rough locations of these basins based on previous work (Uruski, 1992; Barnes and Audru, 1999; Holdgate and Grapes, 2015), which correlate well with the shallow, low velocities imaged in the Cook Strait. Also visible are the Whanganui Basin to the north, and the East Coast Basin. The far-offshore Pegasus Basin (Bland et al., 2015) shows no distinct low-velocity signature due to the lack of resolution past the trench (Figure 5.6A).

Deeper $(8 \mathrm{~km})$ velocity structure in the Cook Strait region shows roughly the same structure as the $3 \mathrm{~km}$ depth slice (Figure 5.6C), suggesting that the underlying tectonic feature that formed these sedimentary basins features extend to depths of 8 $10 \mathrm{~km}$ (Figure 5.6B). In cross section, this relief is much more striking (Figure 5.6E, F). The western boundary, separating the Cook Strait and South Island has a stark gradient of velocities going from more than $4 \mathrm{~km} \mathrm{~s}^{-1}$ to less than $2 \mathrm{~km} \mathrm{~s}^{-1}$ over $50 \mathrm{~km}$ in the upper $10 \mathrm{~km}$ (Figure 5.6F). The northern boundary, separating the Wairau Basin and the Narrows also shows more gradual relief with velocities 

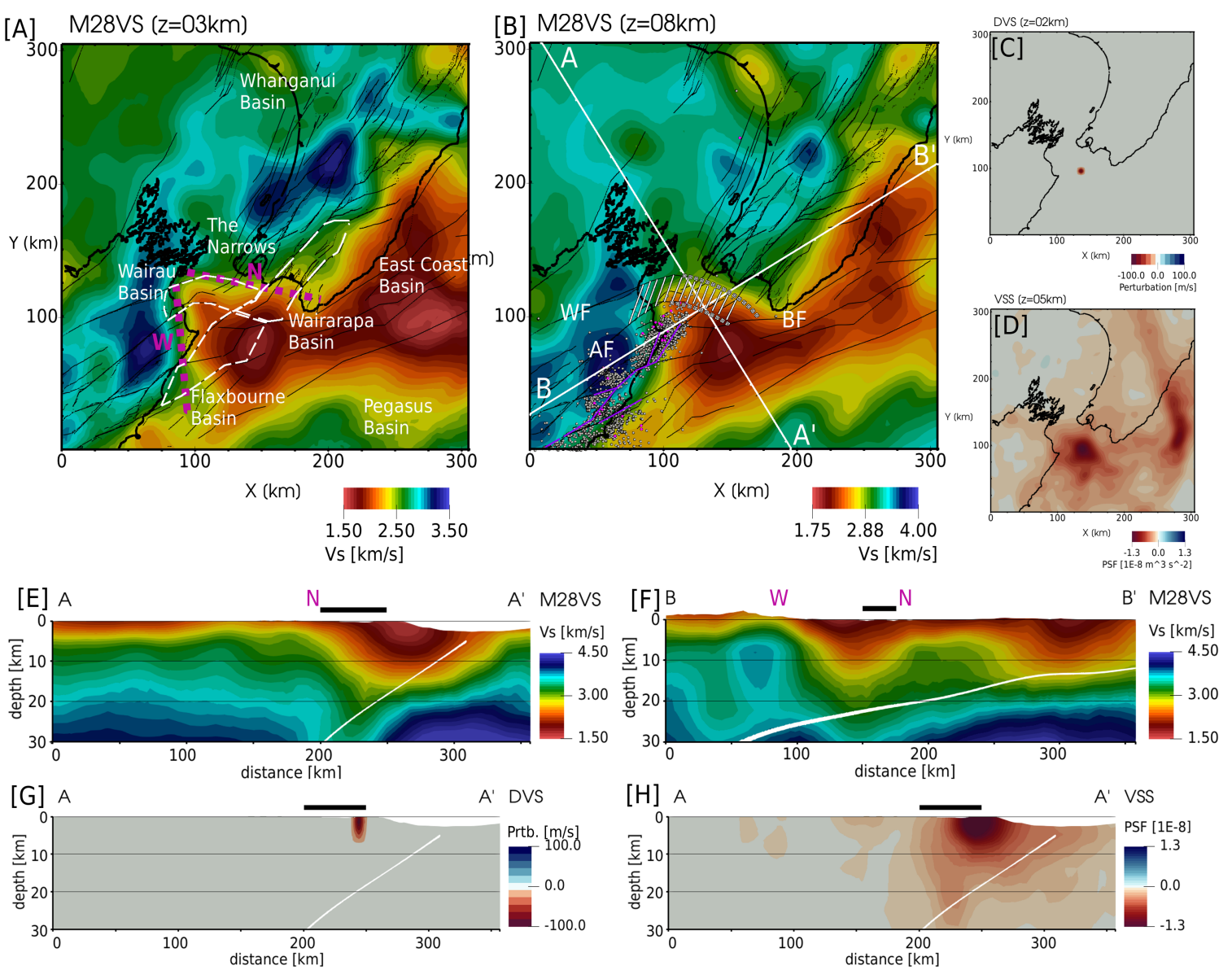

Figure 5.6: Cook Strait velocity gradient and corresponding point spread function. A) M28 $\mathrm{V}_{s} 3 \mathrm{~km}$ depth slice showing low velocities in the Cook Strait, bounded strongly to the north (N) and west (W). Dashed white lines represent approximate locations of Wairau, Wairarapa, and Flaxbourne basins; outlines based on previous work, active faults, and velocity structure. Other labels denote other prominent sedimentary basins in the area. B) M28 $\mathrm{V}_{s} 8 \mathrm{~km}$ depth slice. Relocated Kaikōura aftershocks with depth $>20 \mathrm{~km}$ and $\mathrm{M}>3$ shown as circles. Earthquakes with $\mathrm{M}>5.5$ shown as pink circles (GeoNet). The extent of the crustal transition zone identified by Henrys et al. (2020) marked by hatched pattern between Wairau (WF) and Awatere (AF) and Boo Boo (BF) faults. Surface traces $\mathrm{A}-\mathrm{A}^{\prime}$ and $\mathrm{B}-\mathrm{B}^{\prime}$ correspond to cross sections in $\mathrm{E}-\mathrm{H}$. C) $2 \mathrm{~km}$ depth slice through input $\mathrm{V}_{s}$ perturbation placed in the Cook Strait, with a depth origin at $1 \mathrm{~km}$. D) Point spread function $\mathrm{V}_{S S}$ at $5 \mathrm{~km}$ depth, for input perturbation in (C). E) A- $\mathrm{A}^{\prime}$ cross section through M28 velocity model. F) B-B' cross section through M28 velocity model. G) A-A' cross section through input perturbation shown in (C). H) A- $\mathrm{A}^{\prime}$ cross section through point spread function shown in (D). All cross sections shown to $30 \mathrm{~km}$ depth with 3x vertical exaggeration. White solid lines denotes the plate interface model of Williams et al. (2013), black solid line represents approximate location of crustal transition zone identified by Henrys et al. (2020). 
dropping from approximately $3 \mathrm{~km} \mathrm{~s}^{-1}$ to $2 \mathrm{~km} \mathrm{~s}^{-1}$ across the boundary. These features seem to persist to interface depths, and the western boundary cuts across existing tectonic features such as known active faults (Figure 5.6A)

We use a shallow, negative velocity perturbation to probe resolution in the Cook Strait (Figure 5.6C, G). The resulting point spread function indicates that spatial recovery of the perturbation comes at the cost of significant lateral smearing, due to a lack of stations or events offshore (Figure 5.6D). We take this as an indication that we are only able to resolve long-wavelength features, and that we will not see any distinct boundaries, for example the separation of the offshore basins (Figure 5.6A). It is interesting to note that strong point spread function amplitudes are seen roughly $200 \mathrm{~km}$ to the east of the perturbation (Figure 5.6D). These could be explained by shallow surface waves being guided along the low-velocity basins and reflecting off the continental shelf here, which is characterized by steep bathymetry. These phases may not be well constrained by other sources, and the point spread function indicates that velocity structure in the Cook Strait will have an effect on velocity recovery in the offshore East Coast region. A cross section through the resulting point spread function shows that vertical smearing is not as significant as lateral smearing. Similarly we see negligible mapping of the point spread function onto the strong velocity gradients, which we take as sign that they are not an artefact of the inversion procedure.

The tectonic origins of the Cook Strait, as well as whether faults of the Marlborough Fault System (MFS) and the North Island Dextral Fault Belt (NIDFB) connect beneath the strait are ongoing points of discussion. Lewis et al. (1994) suggest that the Cook Strait developed as sedimentary basins rotated and shifted into line by the oblique, convergent plate boundary. Little and Roberts (1997) provide a potential explanation for tectonic origins, in which arrest of subduction by the anomalously thick Chatham rise caused clockwise rotation of the northern South Island, resulting in internal deformation, pull-apart extension, and strikeslip splaying of the northern Northern Marlborough Domain in the northern South Island (Figure 5.7). Some have concluded that the MFS and NIDFB do not link up (e.g. Carter et al., 1988; Lewis et al., 1994; Little and Roberts, 1997; Pondard and Barnes, 2010), while others believe that the misaligned faults may connect by cutting across known structures at depth (e.g. Barnes and Audru, 1999; Holdgate and Grapes, 2015). 

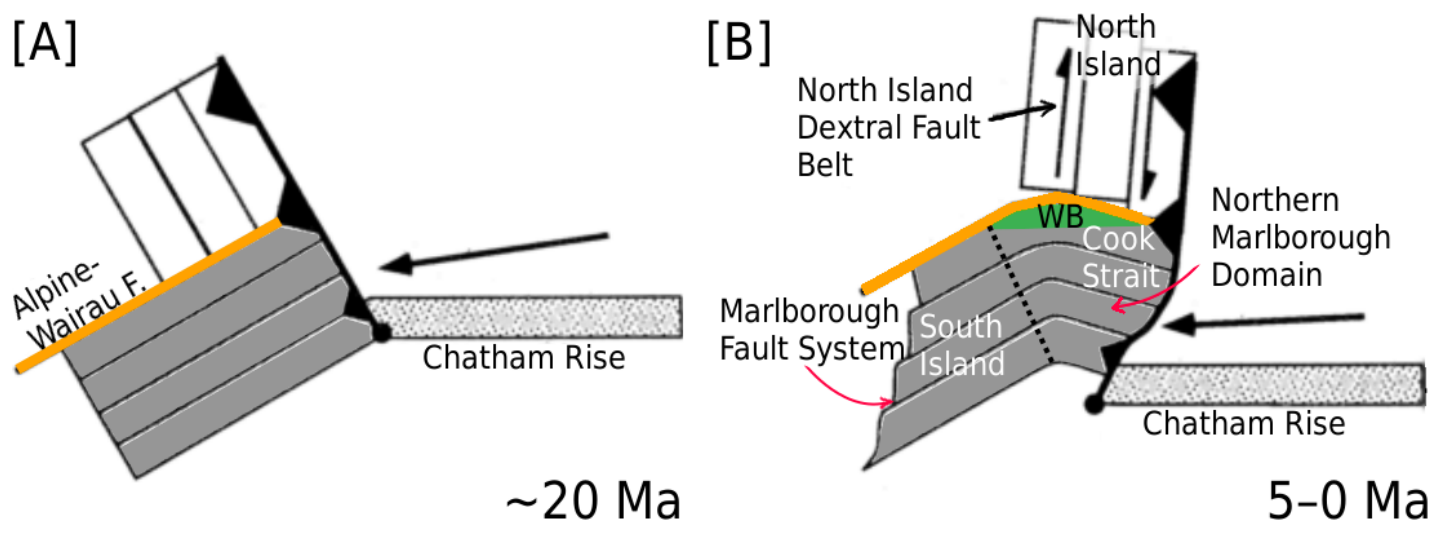

Figure 5.7: One potential tectonic interpretation for the Cook Strait velocity gradient, modified from Little and Roberts (1997). A) Proposed original plate boundary configuration with oblique collision of the Chatham Rise; strike slip motion accommodated by the Alpine-Wairau fault (orange). B) Increased interplate coupling due to the buoyant Chatham rise leads to halt of subduction and pinning of the southern end of the Hikurangi. This results in rotation of the Marlborough Fault System, and southward translation of the Hikurangi margin. Solid block rotation of the Northern Marlborough Domain (NMD) accommodated through localized internal deformation, distributed shear, horsetail splaying of strike-slip faults, and pull-apart basins. The extensional Wairau basin (WB, green area) is bounded to the north by the now-rotated Alpine-Wairau Fault. The dotted black line denotes the western edge of the Northern Marlborough Domain below the South Island. 
More recently Henrys et al. (2020) observed a crustal transition zone, (hatched pattern in Figure 5.6 $\mathrm{B}$ and solid black lines in Figure 5.6E-H) through analysis of targeted $V_{p}$ and $V_{p} / V_{s}$ tomography models, which they propose would inhibit subduction interface rupture propagation to the south. The crustal transition zone of Henrys et al. (2020) coincides with our northern velocity gradient, however they do not observe any boundaries related to the observed western velocity gradient in M28 (Figure 5.6B). Seismicity of the South Island features a point of arrest partway into the Cook Strait, visible in large magnitude $(M>5)$ earthquakes, the 2017 $\mathrm{M}_{\mathrm{w}} 7.8$ Kaikōura mainshock rupture, and resulting, relocated aftershock seismicity (Figure 5.6B; Chamberlain et al., 2021). The point of arrest for seismicity seems to correspond well with the western boundary of our M28 velocity model.

Given the sharp velocity contrasts which are present to depth, cut across known tectonic structures (western boundary), and align with previously identified tectonic features (northern boundary), the origins of the strong velocity gradient in the Cook Strait may correlate with the tectonic origins proposed by Little and Roberts (1997) (Figure 5.7). In this theory, pinning of the southern end of the subduction margin due to its inability to subduct the thick, buoyant Chatham rise (Figure 5.7A), led to southward translation of the Hikurangi margin and clockwise rotation of the Marlborough faults and the Northern Malborough Domain (NMD) (Figure 5.7B). The resulting rigid block rotation of the NMD led to the opening of the Wairau basin against the rotated Wairau Fault to the north, and internal deformation of the block to the west by extensional forces (Little and Roberts, 1997).

We propose that in the context of the framework set by Little and Roberts (1997), our northern velocity boundary may represent the inactive, rotated Wairau fault, which bounds the Wairau basin to the north (Barnes and Audru, 1999; Henrys et al. 2020). The origin of the western boundary remains unclear, however its spatial correlation with a proposed zone of distributed shear (Figure 5.7B), and arrest of seismicity in the Cook strait, suggest a tectonic feature here separating the Cook Strait from the South Island.

\subsubsection{Taupō Volcanic Zone velocity anomalies}

In the Taupō Volcanic Zone, a low-velocity zone $\left(\mathrm{V}_{s}<3 \mathrm{~km} \mathrm{~s}^{-1}\right)$ at $5 \mathrm{~km}$ depth can be seen extending from Ruapehu to the Bay of Plenty in a northeast-southwest 
trend, bounded by high velocities on either side $\left(\mathrm{V}_{s}>3.25 \mathrm{~km} \mathrm{~s}^{-1}\right)$ (Figure 5.8A). Broad scale velocity features in the TVZ correlate well with spatial boundaries related to geophysical and volcanic domains defined in previous studies. The Young TVZ (solid black line in Figure 5.8), which defines a line around the region where intense volcanic and geothermal activity has been seen in the last 350$61 \mathrm{kyr}$ (Wilson et al., 1995), and seems to correlate best with the lowest velocities $\left(\mathrm{V}_{s}<2.75 \mathrm{~km} \mathrm{~s}^{-1}\right)$ seen in this region. Negative gravity anomalies in this region have been inferred to correlate with collapse areas and large caldera complexes (purple outlines in Figure 5.8A; Stagpoole et al. 2020), and may be related to the individual low-velocity lobes seen within the Young TVZ. The wider Taupō Rift (dashed black line in Figure 5.8A; Villamor et al., 2017) also captures the general low-velocity zone that extends beyond the Young TVZ $\left(\mathrm{V}_{s}<3.25 \mathrm{~km} \mathrm{~s}^{-1}\right)$, while the triangular shaped region of positive gravity anomalies known as the Central Volcanic Region (CVR) corresponds to the high-velocity V-shape bounding the low velocities here (white dashed line in Figure 5.8A; Stern, 1985).

A negative velocity perturbation was placed at $1 \mathrm{~km}$ depth in the general location of the Okataina caldera (Figure $5.8 \mathrm{~B}$ ). The resulting point spread function reveals considerable lateral smearing in this region (Figure 5.8 C) with high point spread function amplitudes seen throughout the domain but most strongly centered on the input perturbation. Due to the limited earthquake sources to constrain structure here, coverage of this region is primarily derived from distant surface waves arriving from the south or east. For this reason it is difficult to constrain the locations of velocity anomalies here. We therefore cannot be confident that small-scale velocity anomalies imaged correlate directly to known tectonic or geologic features. For example, the low-velocity anomaly that protrudes outside the eastern border of the Young TVZ seems to correlate to the high amplitude point spread function arriving from the south, and may be the result of lateral smearing in the north-south direction. In cross-section, we see that the input perturbation extends to $5 \mathrm{~km}$ depth (Figure 5.8 E). The resulting point spread function seems to map the strongest amplitudes with good vertical resolution, however there is smearing visible down to $20 \mathrm{~km}$ depth (Figure 5.8 F). Lateral smearing is also clearly visible here.

The central TVZ, between the Taupō and Okataina calderas (Figure 5.8), is an exceptionally productive region of silicic volcanism (Wilson et al., 2009). In con- 

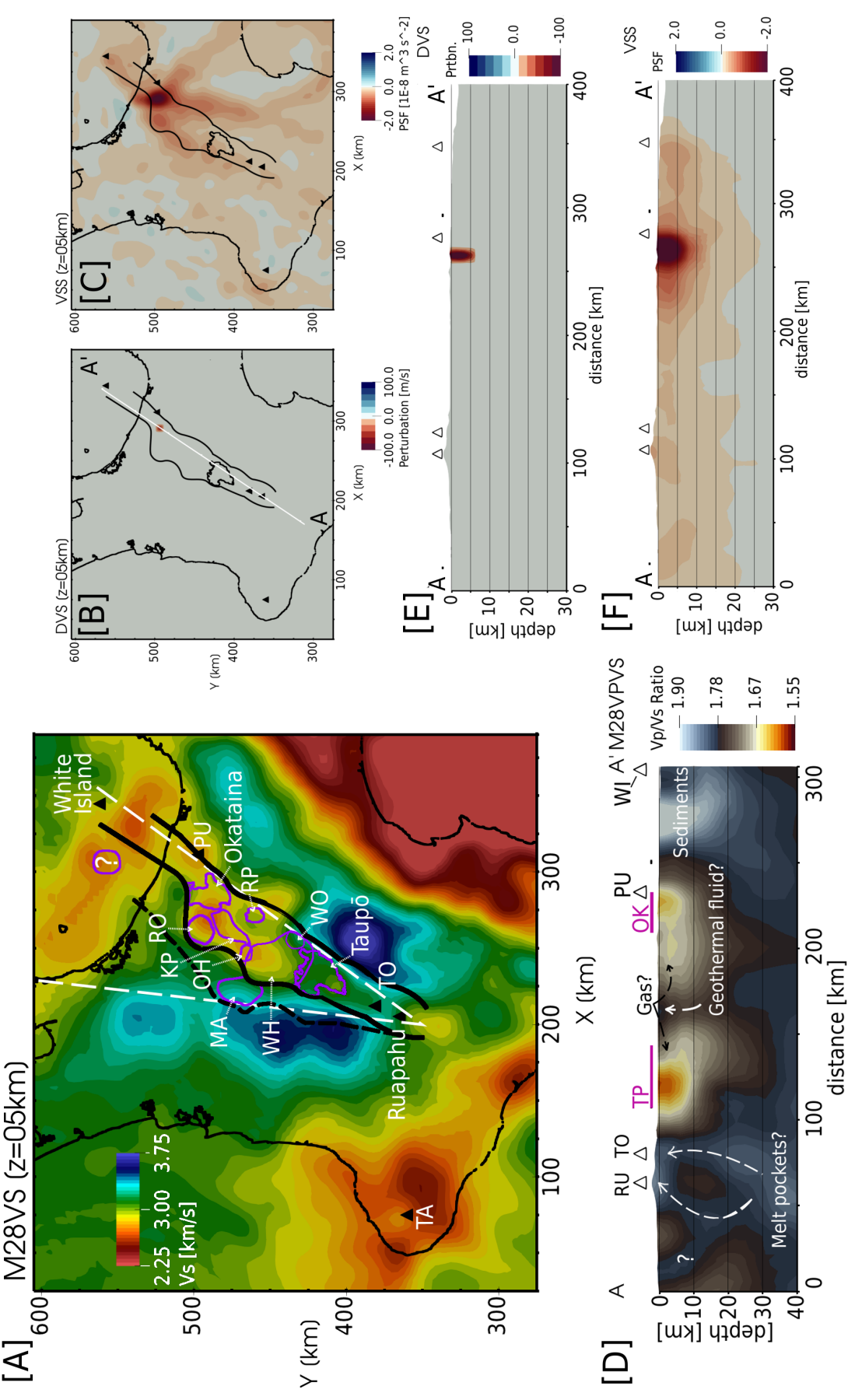
trast, back-arc andesitic volcanism is seen in the northern and southern TVZ: from Okataina caldera towards White Island in the north, and from Ruapehu to the southern end of Taupō in the south (Wilson et al., 1995). This region has previously been studied with geophysical methods. For example, magnetotelluric studies have imagined a high conductivity plume-like structure beneath the rift axis between Taupō and Okataina (Heise et al., 2010), while a study of seismic attenuation identified low $Q_{s}$ values underlying caldera structures in the central rift structure (Eberhart-Phillips et al., 2020a).

We use the $V_{p} / V_{s}$ ratio to explore the TVZ due to its sensitivity to fluid presence (Figure 5.8D); high $\mathrm{V}_{p} / \mathrm{V}_{s}$ have been inferred to indicate volumes of partial melt and the presence of magma chambers (Koulakov, 2013), although it should also be sensitive to other fluid inclusions, such as geothermal fluids at depth. Similarly, low $V_{p} / V_{s}$ has been used to indicate the presence of over-pressured gas in a volcanic system (Wilks et al. 2020). As with the interpretation of $V_{p} / V_{s}$ in Section 5.2.2, we note the possibility of apparent $V_{p} / V_{s}$ structures arising from differences in resolution of $V_{p}$ and $V_{s}$ within our inversion. In addition to these interpretations, further analysis may be required to determine to what extent resolved $\mathrm{V}_{p} / \mathrm{V}_{s}$ structure in the TVZ is affected by resolution differences between $\mathrm{V}_{p}$ and $\mathrm{V}_{s}$.

The rift-parallel $V_{p} / V_{s}$ cross section shows an interesting heterogeneous distribution correlating to variation in the types of volcanism seen at the TVZ. To the south, columns of high $\mathrm{V}_{p} / \mathrm{V}_{s}(>1.8)$ are seen rising to the andesitic Ruapehu and Tongariro volcanoes, which may represent a blurred, long-wavelength depiction of distributed melt pockets feeding these volcanoes (Figure 5.8D). Below Taupō and Okataina calderas, we might expect to see melt bodies around 5-15 km depth (Barker et al., 2020), imaged as high $\mathrm{V}_{p} / \mathrm{V}_{s}$ signatures signifying a high percentage of partial melt. In contrast what we observe is shallow, low $\mathrm{V}_{p} / \mathrm{V}_{s}(<1.6)$ signatures below each caldera (Figure 5.8D). We propose that the lowest $V_{p} / V_{s}$ may actually be found above $5 \mathrm{~km}$ depth and related to over pressured gas in the shallow crust, whose signature has been blurred to depth. In between the two calderas, high $\mathrm{V}_{p} / \mathrm{V}_{s}$ may relate to the presence of geothermal fluids, evidenced at the surface by active geothermal fields in this region (Chambefort et al., 2014). North of the coast towards White Island, $\mathrm{V}_{p} / \mathrm{V}_{s}$ rises above values of 1.9 , which is likely the result of low-velocity, fluid saturated sediments dominating structure 
here (Figure 5.8D).

\subsubsection{Implications of strong velocity changes}

The large $( \pm 30 \%)$ velocity changes recovered in M28 with respect to the initial NZ-Wide2.2 velocity model may have significant impacts on studies that rely on 3D velocity structure as input, including but not limited to: inversions of shallow subduction slow slip events, earthquake relocations, ground motion prediction simulations, and estimations of seismic hazard.

Williams and Wallace (2018) use the NZ-Wide velocity model to generate Green's functions and estimate the magnitude of slow slip events on the Hikurangi subduction interface. They find that introducing heterogeneous elastic properties has extremely large effects with respect to a homogeneous interface, increasing seismic potency by $58 \%$ or more. With our revised M28 $\mathrm{V}_{s}$ model we would expect heterogeneous changes in the Lamé parameters $\lambda$ and $\mu$, which should have significant impact on the resulting slip estimations. For example, assuming a constant density, $\mathrm{V}_{s}$ changes as much as $\pm 30 \%$, would result in almost $\pm 70 \%$ change in the shear modulus, $\mu$, which is on the order of changes introduced between the homogeneous and heterogeneous velocity model cases in Williams and Wallace (2018). If density is allowed to change, shear modulus changes would be significant but not as severe. Additionally, increased offshore resolution of the shallow subduction interface should increase spatial heterogeneity of interface properties, leading to changes in estimations in expected slip, which would consequently have an effect on estimated slip budget on the Hikurangi (Williams and Wallace, 2018).

Earthquake relocations are an important method for constraining relative and absolute depths and locations of seismic events, allowing earthquake catalogs to be used to map fault structures (Lanza et al. 2019), infer pore fluid pressures, or spatially constrain large scale tectonic features (Reyners et al., 2017). Methods like nonlinear location inversion (Lomax et al., 2000) can use input 3D velocity models to search for optimum earthquake locations, with 3D structure directly constraining location accuracy. The NZ-Wide velocity model has repeatedly been employed in earthquake relocation studies (e.g. Bannister et al., 2011; Reyners et al., 2011; Lanza et al. 2019), and we would expect that changes introduced in this inversion should have an effect on inferred earthquake locations. For example, revised 
offshore velocities should improve locations of near-offshore earthquakes, while increased resolution of shallow structure may help to better constrain earthquake depths.

Ground motion simulations can be used to provide constraints on expected ground shaking for large potential earthquakes (e.g. Graves et al., 2011; Bradley et al., 2017). In these simulations, the underlying velocity model controls 3D wave propagation effects, such as amplified shaking in sedimentary basins or directivity caused by topography or subsurface structure. In the South Island, New Zealand, for example, ground motion simulations of a large Alpine fault rupture show significant rupture directivity and basin-generated surface waves that result in notable increases in peak ground velocities (Bradley et al. 2017). Similarly, the adjoint tomography model of Tape et al. (2010) has been used to constrain strong ground motion of potential fault ruptures to estimate seismic hazard in Southern California (Graves et al., 2011).

Through the increased resolution of shallow crustal structure in M28, particularly in onshore-offshore sedimentary basins, and of strong velocity gradients related to geologic and tectonic boundaries, we expect our updated velocity model to have a significant impact on ground motion simulations, and consequently seismic risk estimates. For example, observed long-duration ground shaking in offshore regions should be more accurately captured by the updated velocity structure (Kaneko et al., 2019). In addition, Ellis et al. (2017) show that velocity gradients at depth can be used to identify previously unmapped faults which may host large, damaging earthquakes. The updated velocity model, resultant wave propagation simulations, and predictions of faults and ground shaking, will undoubtedly have an impact on the estimation of seismic hazard throughout New Zealand.

\subsection{Conclusions}

Comparisons of the final velocity model with geologic cross sections (Figure 5.2) show that shallow vertical resolutions are well constrained. Point spread tests reveal that the resolved velocity anomalies are robust features of the final velocity model, with varying degrees of lateral smearing due to the primary use of surface wave measurements. 
We interpret the most striking velocity changes in the M28 final $V_{s}$ model with respect to known geology and tectonics. Shallow $\mathrm{V}_{s}$ velocity structure correlates well with the locations of different geologic basement terranes such as the Rakaia, Pahau, Kaweka, and Waioeka terranes in the east, and the Morrinsville terrane in the west. Along-strike $V_{p} / V_{s}$ structure shows increased heterogeneity from north to south, which contrasts with current interpretations that variations in terrane permeability control interface locking. Below the east coast of the North Island we image two previously unidentified subducted seamounts and discuss their connection with terrain features and anomalous observations in these regions. In the Cook Strait we image steeply sided, deep sedimentary basins, with strong velocity contrasts between the Cook Strait and North and South Islands. The velocity gradients cut across known structures and show correlation with previously proposed tectonic features, as well as an anomalous termination point of seismicity observed in the Cook Strait. In the Taupō Volcanic zone we image slow $\left(\mathrm{V}_{s}<3 \mathrm{~km} \mathrm{~s}^{-1}\right)$, shallow velocities that, in general, correlate spatially with low-gravity anomalies inferred to be caldera locations. $V_{p} / V_{s}$ structure at depth may correlate with crustal magmatic activity. Point spread tests are used to constrain the robustness of features, and indicate a varying amount of lateral uncertainty due to smearing.

This new velocity model illuminates previously unidentified features in the crust and provides further seismological constraints on enigmatic tectonic properties of the Hikurangi subduction zone. Pronounced velocity changes revealed in the final model will have significant effect on future geophysical studies which make use of $3 \mathrm{D}$ velocity structure, including earthquake relocations, interface slip characteristics, and estimations of seismic hazard. 


\section{Chapter 6}

\section{Synthesis}

The overarching goal of this thesis was to develop and interpret a high resolution velocity model for the North Island of New Zealand using adjoint tomography. We began by assessing an existing 3D ray-based tomography model of New Zealand for its suitability in an adjoint tomography inversion, using waveform misfits and checkerboard synthetic inversions (Section 3.2). In conjunction with this analysis, we developed a set of procedures and automated tools for performing adjoint tomography in New Zealand and in other domains (Section 3.3). The datasynthetic misfit from the initial velocity model showed a systematic time shift between earthquake observations and corresponding simulations (Figure 3.4), while synthetic inversions showed that New Zealand source-receiver distributions could be used to improve the starting velocity model (Section 3.4). This work has also led to deployment of a temporary seismic network (BEACON) which targeted improved coverage over the geodetically inferred locked-to-creeping transition of the Hikurangi subduction margin (Section 2.3).

We performed a full-scale tomographic inversion using up to 1800 unique sourcereceiver pairs and 4-30 s waveforms (Chapter 4). The updated velocity model, M28, shows significant waveform improvement and resolution of strong crustal heterogeneity (Section 4.4). We interpreted the most striking velocity changes that have illuminated previously unidentified features, and discussed potential tectonic origins and implications for our understanding of the Hikurangi subduction zone (Chapter 5). The following section details how the research objectives put forth at the beginning of this thesis have been addressed (Section 6.1), and 
proposes future research directions that may stem from this work (Section 6.2).

\subsection{Revisiting research objectives}

In the following section we revisit the research objectives set out in Section 1.1. and evaluate how each has been addressed throughout this thesis. We repeat each research objective in italicized text for clarity.

1. What are the best practices for performing adjoint tomography in New Zealand?

In Chapter 3, we described a set of procedures that reflect one approach to performing adjoint tomography in New Zealand (Section 3.2), which makes use of existing and newly developed open-source tools (Section 3.3). In Chapter 4, we apply this workflow to a full-scale North Island inversion. Here we highlight some of the more important points relevant to this research objective.

Data are important. The computational cost of adjoint tomography scales with the number of events (Section 2.1.5), so each event should maximize the number of measurements it contributes to the dataset. Similarly the maximum number of seismic stations should be included and unique sourcereceiver paths should be given preference. However, as discussed in Chapter 4, repeat stations and events lead to lopsided contributions to the inversion, and these should be treated with care (Section 4.2). We note that event and station declustering or weighting is important for a well-behaved inversion. In summary: less is more, and more is not always better.

Meshing demands respect. As discussed in Section 4.3.3, meshing had an undesired effect on the inversion. Although this was deemed unimportant for interpretations, additional care during meshing might have mitigated this. Choices such as coinciding depths of doubling and tripling layers with the majority of earthquakes ( $\sim 15 \mathrm{~km})$, and squeezing mesh bounds tight around the dataset, may have resulted in unwanted numerical effects that would require exhaustive testing to understand the impacts of (Section 4.3.1). To wit, exercise caution when devising a mesh, and be more conservative than you think you should be.

"Never spend 6 minutes doing something by hand when you can spend 6 
hours failing to automate it. "11 NeSI's high performance computers were sufficient for the task at hand and we encountered no bottlenecks in terms of available compute power (Section 4.4.4). However, queue times increased consistently during this work, and long wait times were always a looming concern. Pertinent advice is to run simulations overnight and through the weekend, and not be dependent on something running asap. A moderate degree of automation makes this increasingly feasible.

2. How does the application of adjoint methods improve the current ray-based tomography model of New Zealand?

The application of adjoint tomography to a ray-based traveltime tomography model has improved overall data-synthetic time shifts for observations and simulations of large magnitude, regional earthquakes (Section 4.4). Better fits of long-period waveforms for stations around the North and South Islands (Section 4.4.2) have resulted in recovery of $\pm 30 \%$ velocity changes with respect to initial velocity values, and illumination of shallow velocity features previously unseen in the initial ray-based model (Section 4.4.1). The use of surface wave measurements in adjoint tomography has led to marked resolution of shallow $\mathrm{V}_{s}$ and $\mathrm{V}_{p} / \mathrm{V}_{s}$ structure (Figure 4.7), with particularly drastic improvements in near-offshore regions where resolution of the initial model was less well-constrained (Section 4.5).

3. Do the resulting variations in seismic velocities show similar changes to other applications of adjoint tomography?

We observe similar magnitudes of velocity changes with respect to other applications of adjoint tomography (Chapter 4). In Section 4.4.1, we note stark seismic velocity changes with respect to the initial model, that show similar changes as seen in previous applications of adjoint tomography (Figure 4.4, namely $\pm 30 \%$ velocity changes from the starting model as in Tape et al. (2010). In this work we recover peak values of $\pm 33 \% \mathrm{~V}_{s}$ changes with respect to starting values (Section 4.4.1). In $\mathrm{V}_{p}$ we see up to $\pm 25 \%$ changes (Figure E.1). These velocity changes strongly correlate with the very low velocities in the offshore accretionary prism and sedimentary basins, as well as two strong, high-velocity anomalies below the east coast of the North Island

${ }^{1}$ Zhuowei Zhang, Twitter 
(Section 4.4.1).

4. What velocity anomalies are revealed that were not resolvable with previous models and what are the physical interpretations of such anomalies?

We identify multiple striking velocity anomalies associated with our new velocity model (Section 4.4.1). In Chapter 4, we spatially distinguish these striking velocity changes as features $\mathrm{A}-\mathrm{E}$ in Section 4.4.1. These velocity anomalies are not present in the initial model. In Chapter 5, we interpret these features as: 1) two deeply subducted seamounts below the east coast of the North Island (Section 5.2.3); 2) very deep, steeply sided sedimentary basins in the Cook Strait that form a stark contrast in velocity structure between the North and South Islands, and potentially signify a tectonic boundary that cuts across existing structure here (Section 5.2.4); 3) distinct low-velocity anomalies in the Taupō Volcanic Zone, as well as underlying heterogeneous $\mathrm{V}_{p} / \mathrm{V}_{s}$ structures at depth, which may relate to volcanic activity in the shallow crust (Section 5.2.5).

5. What are the structural differences, manifest as seismic velocities, between the northern and southern Hikurangi margins, and what are their physical interpretations?

In Chapter 5 we assess along-strike material properties of the Hikurangi margin (Section 5.2.2), and find that our updated $\mathrm{V}_{p} / \mathrm{V}_{s}$ model contradicts previous hypotheses that basement terrane boundaries influence fluid distribution and consequently geodetically observed locking of the southern Hikurangi margin (Figure 5.3). Our velocity model shows strong along-strike heterogeneity, which may be linked to the deep subduction of seamounts in the northern Hikurangi margin (Section 5.2.3), and corresponding elastic and inelastic deformation of the upper plate resulting in the observed difference in slip behavior along-strike of the Hikurangi margin.

\subsection{Potential for future work}

\subsubsection{A New Zealand adjoint tomography model}

A natural extension of this work is to apply adjoint tomography to the entire New Zealand domain. In this thesis we specifically target the Hikurangi subduction 
zone below the North Island of New Zealand (Section 4.2), but the NZ-Wide2.2 velocity model is defined for the entire New Zealand domain (Eberhart-Phillips et al., 2020b). A New Zealand wide adjoint tomography inversion may follow the initial model assessment laid out in Chapter 3 , and perform an inversion using the automated tools developed in Section 3.3. An ideal target domain would include the entire New Zealand region in a single inversion, however computational costs could become a limitation. An alternative approach would be to perform separate North Island (Figure 6.1) and South Island (Figure 6.2) inversions, and combine the resulting velocity models after the fact. These two avenues are discussed below.

A unified New Zealand wide velocity model, which includes measurements from source-receiver paths that cross between the North and South Islands, would be the optimal design for a New Zealand wide adjoint tomography inversion. However, computational limitations may prove a bottleneck here. In a standard Cartesian coordinate system (e.g. UTM), New Zealand strikes at a roughly $45^{\circ}$ angle, resulting in an abundance of ocean domain within the resulting mesh. Similarly, distant source-receiver paths ( $>500 \mathrm{~km}$ ) through this domain would significantly increase simulation times. Rotating the New Zealand domain, as done with the NZWide2.2 velocity model (Eberhart-Phillips et al., 2020b), may reduce the amount of unused domain space, but also increases problem complexity. Custom made meshes could also be designed, such as those that only provide simulation domain for the largest epicentral distance of a given source, or incorporating the idea of wavefield adapted meshes as in Thrastarson et al. (2020). These solutions trade computational cost for setup cost, and may be worthwhile for the resultant unified tomography model. In addition, the use of graphics processing units (GPUs) would significantly improve computational performance and increase feasibility of a unified New Zealand inversion; GPUs were not used in this work due to a lack of available resources.

Alternatively, separate North and South Island inversions could be performed and resulting models combined post-hoc. A complete North Island inversion may extend the NZ-North domain (Section 3.2) to the north and east (Figure 6.1). Velocity structure in our domain is truncated east of East Cape (Figure 6.1), and interesting velocity features here are not fully resolved, such as: potential seamounts further north connected to the Ruatoria re-entrant (e.g. Lewis et al., 1998; Collot et al. 2001), and the low-velocity accretionary wedge responsible for ultra-long 
coda waves in the northeastern North Island (Kaneko et al., 2019). A recent large $\left(\mathrm{M}_{\mathrm{w}} 7.1\right)$ earthquake $125 \mathrm{~km}$ east of East Cape $\mathrm{f}^{2}$ has resulted in a substantial number of aftershocks with appropriate magnitude and depth ranges for use in adjoint tomography, which may provide increased resolution far offshore (Figure 6.1). We have made preliminary efforts to design a full North Island mesh, accurate to $2 \mathrm{~s}$ periods and containing roughly 1000000 elements, which could be used to target a full North Island inversion (Figure 6.1).

The South Island contains sparse, but uniform broadband seismometer coverage provided by the GeoNet permanent seismic network (Figure 6.2). Additional stations here can be used to enhance coverage. For example the Southern Alps Long and Skinny Array (SALSA) is an anticipated large-scale broadband seismometer network that will be deployed to study the Alpine fault (Figure 6.2). Additional short-period networks have been installed to study Alpine Fault seismicity (Boese et al., 2012, Chamberlain et al., 2014). Broadband stations have also been deployed at the Puyseger subduction zone during the South Island Subduction Initiation Experiment ${ }^{3}$ (SISIE) (Figure 6.2). Interesting tectonic features that could be resolved here have been discussed in previous South Island tomography studies and include: the Puyseger subduction zone, the transition from oblique subduction to oblique transpression in the Marlborough fault system (Section 5.2.4), continental root structure beneath the Alpine fault, and shallow crustal structure of the Canterbury basin (Eberhart-Phillips and Reyners, 1997; Eberhart-Phillips and Bannister, 2010; Eberhart-Phillips et al., 2018).

\subsubsection{Targeted geophysical studies}

We have put forth various tectonic interpretations of observed seismic velocity changes, including: 1) subducted seamounts below the east coast of the North Island (Section 5.2.3), 2) a steeply sided sedimentary basin forming a potential tectonic boundary between the North and South Islands (Section 5.2.4), 3) shallow low-velocity anomalies and deep high $\mathrm{V}_{p} / \mathrm{V}_{s}$ structures below the TVZ, related to volcanic activity in the crust (Section 5.2.5). Each of these features has been corroborated with other geophysical studies, however precise resolution of amplitudes, scale, locations, and depths remain unclear. Further targeted geophysical studies

\footnotetext{
${ }^{2}$ https:/ / www.geonet.org.nz/earthquake/2021p169083

${ }^{3} \mathrm{http}$ :/ / geoprisms.org/education/report-from-the-field/new-zealand-2018/sisie-spring2018/
} 

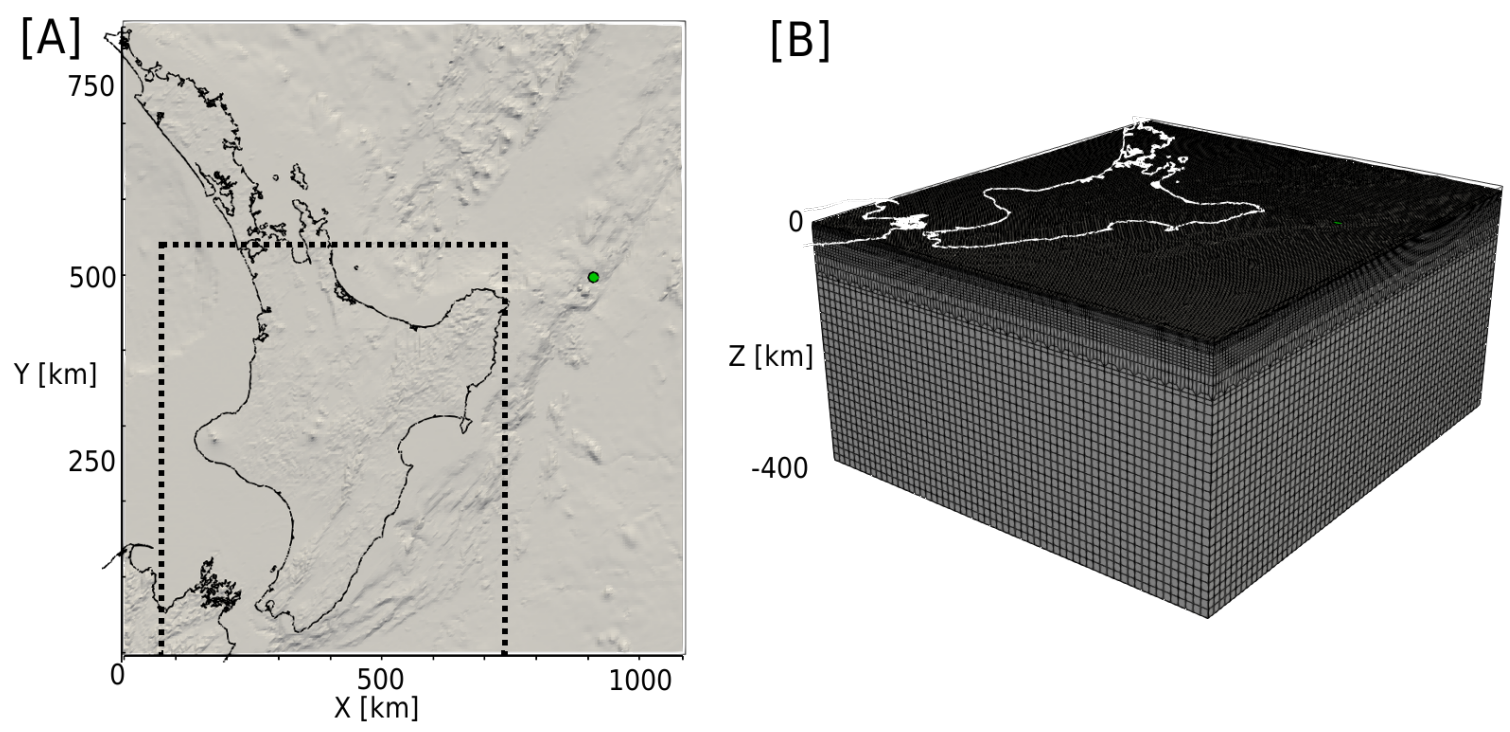

Figure 6.1: Full North Island mesh, accurate to $2 \mathrm{~s}$ periods, containing approximately 1000000 elements. A) Map view with 3X vertical exaggeration to highlight elevation differences. Topography and bathymetry defined by SRTM-30P (Becker et al., 2009). Green circle shows epicentral location of the $\mathrm{M}_{\mathrm{w}} 7.1$ Te Araroa earthquake, which produced a substantial number of aftershocks that can be used for adjoint tomography (Section 6.2.1). Dotted line shows the approximate bounds of the domain used in this study. B) Mesh viewed from side, showing mesh refinement at depth.

may help to corroborate these interpretations.

Trench-parallel onshore-offshore seismic lines through Māhia Peninsula and Pōrangahau would be an ideal method for probing the existence of deeply subducted seamounts; marine seismics have been an excellent tool for finding seamounts in the shallower offshore domain (Barnes et al., 2010; Bell et al., 2010). Targeted magnetic and gravity surveys may also provide useful evidence. In the Cook Strait, marine seismics may help pin down the western and northern velocity gradient (Section 5.2.4). Earthquake relocation studies using this updated velocity model may also help constrain the existence of seamounts, and the offshore limit of seismicity in the Cook Strait (e.g. Lanza et al., 2019); we discuss this further in Section 6.2.3. In the TVZ, targeted active seismics may help confirm if the $V_{p} / V_{s}$ ratios we observe below Taupō and Okataina are related to proposed overpressured gas and distributed melt pockets (Section 5.2.5). Local earthquake tomography or ambient noise tomography may help constrain shallow crustal structure here by leveraging smaller magnitude, localized events that were not suitable for adjoint tomography. 


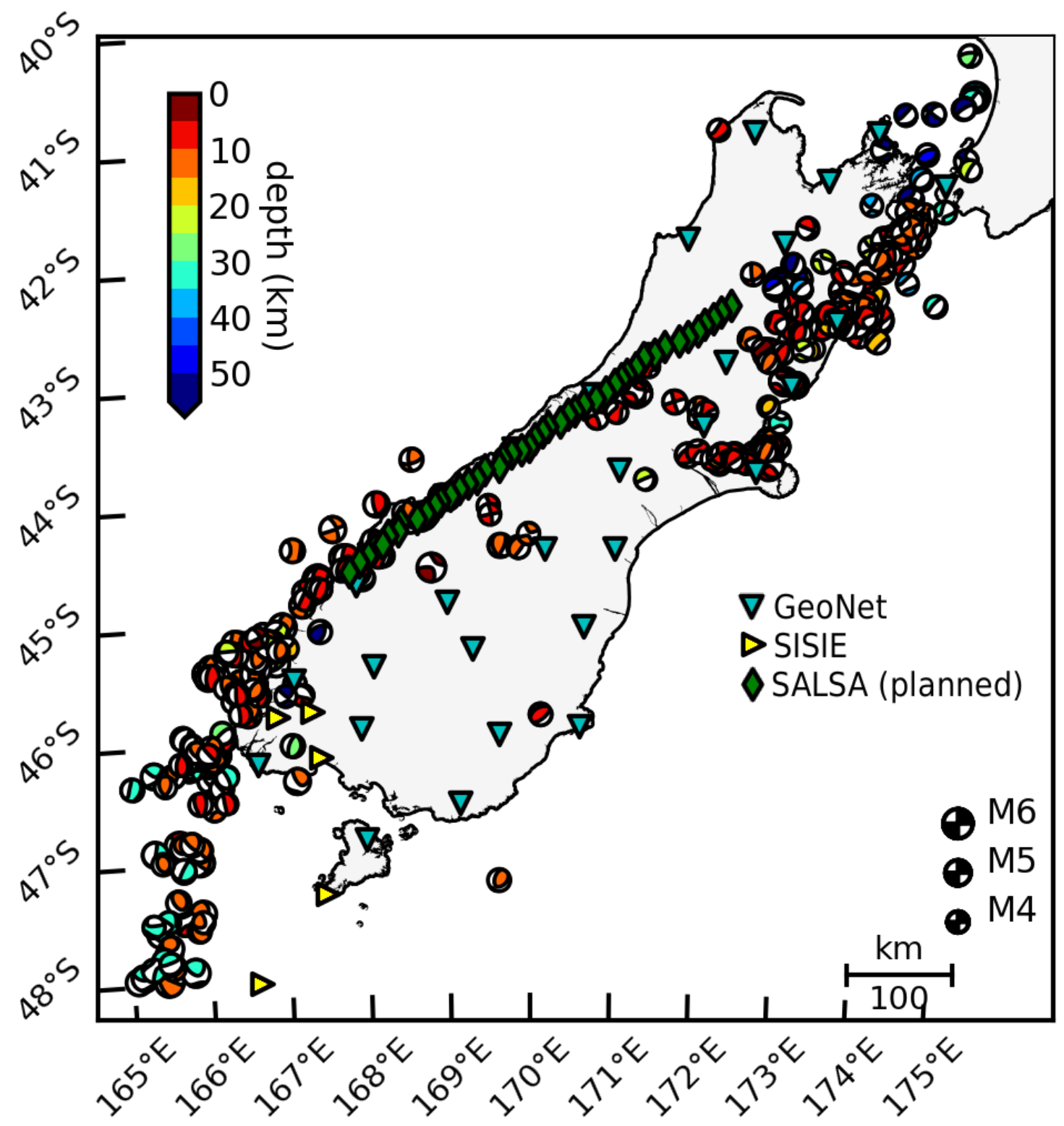

Figure 6.2: Broadband seismic stations and more than 400 earthquakes for a potential South Island adjoint tomography inversion. Earthquake moment tensors from GeoNet and are shown only for suitable magnitudes and depths for adjoint tomography $(\mathrm{Z}<$ $\left.60 \mathrm{~km} ; 4.5 \leq \mathrm{M}_{\mathrm{w}}<6.0\right)$. Stations from the GeoNet permanent seismic network shown as inverted blue triangles. Yellow triangles show broadband station locations from the South Island Subduction Initiation Experiment (SISIE). Green diamonds show planned station locations for the Southern Alps Long Skinny Array (SALSA). 


\subsubsection{Earthquake relocation}

Accurate locations of seismic events are critically dependent on the accuracy of the underlying velocity model. With our new velocity model, which is shown to improve 3D synthetic waveform fits (Section 4.4.2), and more accurately resolve shallow structure (Section 4.5), an earthquake relocation study can be undertaken using small magnitude earthquakes $\left(1.5<\mathrm{M}_{\mathrm{w}}<4.0\right)$ and non-linear earthquake location algorithms (e.g. Lomax et al., 2000). Relocated seismicity is commonly used in conjunction with tomographic models to study seismic structure, fluid distributions, and geometries of deep tectonic features (e.g. Reyners et al., 2017). With a new relocated seismicity catalog, similar studies could be performed, targeting for example: new estimates on the geometry of the Hikurangi plate interface, fluid distributions along the interface, or additional constraints on subduction-related anomalies such as the inferred seamounts below Māhia Peninsula and Pōrangahau (Section 5.2.3).

\subsection{Concluding statement}

This thesis has culminated in a new velocity model of the North Island of New Zealand, and illuminates anomalous tectonic features related to the Hikurangi subduction zone. This is the first application of adjoint tomography to the New Zealand region, and has resulted in resolution of previously unidentified velocity features around the North Island of New Zealand. The results of the work in this thesis include: a new seismic dataset from the BEACON deployment, an open-source automated workflow for adjoint tomography, and a newly revised $3 \mathrm{D}$ velocity model for the North Island of New Zealand. The work outlined herein serves to improve our ever-expanding understanding of the Hikurangi subduction zone. 


\section{Appendix A}

\section{Windowing parameters}

The following table contains the input windowing parameters used to control the windowing characteristics of the Flexwin algorithm through the Python package, Pyflex. Time window selection is explained in Section 3.3 . 


\begin{tabular}{|c|c|c|c|c|c|c|c|c|c|c|c|c|c|c|c|c|c|}
\hline પ્ & + & ले & H & $\begin{array}{l}\mathrm{L} \\
0 \\
0 \\
0\end{array}$ & $\begin{array}{l}8 \\
8 \\
0 \\
0\end{array}$ & 0 & $\stackrel{n}{-}$ & 0 & $\grave{o}$ & $m$ & $\stackrel{L}{\infty}$ & $\stackrel{\mathscr{L}}{\mathrm{N}}$ & $\begin{array}{l}\stackrel{L}{N} \\
\stackrel{N}{N}\end{array}$ & $\sigma$ & L & $\neg$ & $\neg$ \\
\hline טلِ & 6 & ले & $m$ & $\mid \begin{array}{l}\infty \\
0 \\
0\end{array}$ & $\stackrel{0}{0}$ & $\infty$ & $\stackrel{n}{-}$ & 0 & $\stackrel{\infty}{0}$ & $N$ & $\infty$ & $N$ & $\stackrel{L}{i}$ & $\mathcal{I}$ & 10 & $\stackrel{\stackrel{L}{O}}{\stackrel{\leftrightarrow}{\sim}}$ & $\neg$ \\
\hline 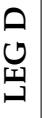 & $\infty$ & ले & $m$ & $\breve{0}$ & $\hat{0}$ & $\infty$ & $\stackrel{\text { ?ִ }}{\rightarrow}$ & 0 & $\widehat{0}$ & $N$ & + & $N$ & $\stackrel{L}{i}$ & $\approx$ & 10 & $\stackrel{\longrightarrow}{\longrightarrow}$ & -1 \\
\hline 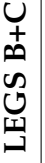 & $\stackrel{9}{\circ}$ & लि & $m$ & $\overrightarrow{0}$ & $\hat{0}$ & $\stackrel{?}{\sim}$ & $N$ & 0 & $\hat{0}$ & $N$ & $\infty$ & $N$ & $\stackrel{L}{\mathrm{i}}$ & $\approx$ & 10 & $\stackrel{\sim}{\longrightarrow}$ & $\neg$ \\
\hline 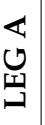 & $\stackrel{\circ}{\sim}$ & लि & $\stackrel{\llcorner}{\mathrm{N}}$ & $\begin{array}{l}\infty \\
0 \\
0 \\
0\end{array}$ & $\hat{o}$ & $\simeq$ & $\stackrel{L}{i}$ & 0 & $\hat{o}$ & $N$ & $\rightarrow$ & $N$ & $\infty$ & $\stackrel{9}{\sim}$ & 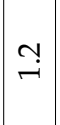 & 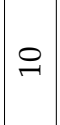 & $\neg$ \\
\hline $\begin{array}{l}\mathbf{N} \\
\mathbf{Z}\end{array}$ & 우 & கి & $\stackrel{n}{n}$ & $\begin{array}{l}0 \\
\dot{0}\end{array}$ & $\hat{o}$ & 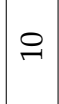 & $N$ & $H$ & $\hat{o}$ & $N$ & $\infty$ & $N$ & $\stackrel{\llcorner}{\mathrm{i}}$ & $\approx$ & $\infty$ & in & 0 \\
\hline $\begin{array}{l}\mathbf{c} \\
\mathbf{d} \\
\mathbf{N} \\
\mathbf{z}\end{array}$ & $N$ & लि & 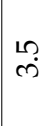 & $\ddot{0}$ & $\hat{o}$ & $\infty$ & $N$ & 0 & $\hat{0}$ & 10 & $\infty$ & $N$ & $\stackrel{L}{i}$ & $\mathcal{Z}$ & $\infty$ & in & 0 \\
\hline $\begin{array}{c}\mathbf{c} \\
\text { d } \\
\mathbf{N} \\
\mathbf{z}\end{array}$ & $\stackrel{9}{\circ}$ & கి & $\stackrel{10}{\infty}$ & $\ddot{0}$ & $\hat{o}$ & $\infty$ & $N$ & 0 & $\hat{o}$ & $N$ & $\infty$ & $N$ & $\stackrel{L}{\mathrm{i}}$ & $\mathcal{I}$ & $\infty$ & in & 0 \\
\hline 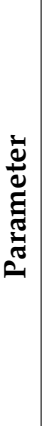 & 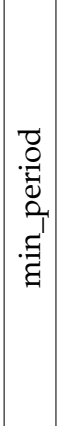 & 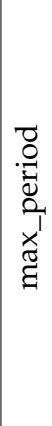 & 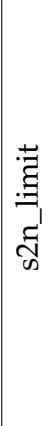 & 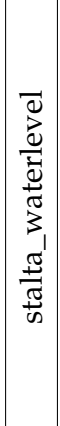 & 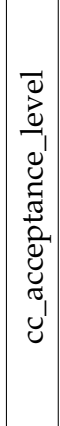 & 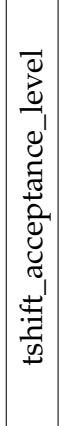 & 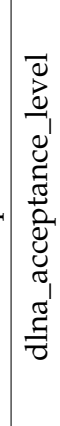 & 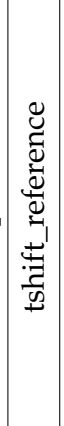 & $\begin{array}{l}0 \\
0 \\
0\end{array}$ & $\sigma^{\prime}$ & $\mid \begin{array}{c}\tilde{m}_{1} \\
u^{\prime}\end{array}$ & $\begin{array}{l}\hat{m}_{1} \\
\mathrm{u}^{\prime}\end{array}$ & $\begin{array}{l}\text { F } \\
0_{1}^{\prime}\end{array}$ & $\vec{f}_{1}$ & 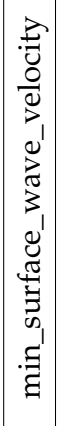 & 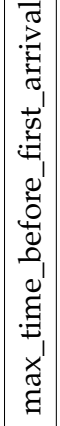 & 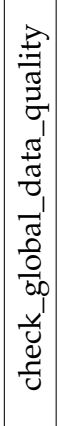 \\
\hline
\end{tabular}

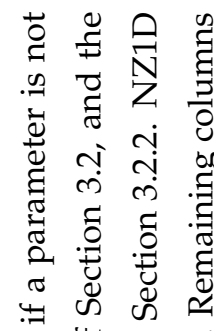

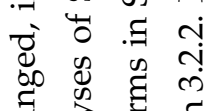

ปี त

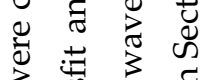
3 氙 3.9 节 荧

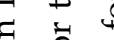

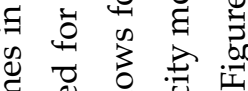

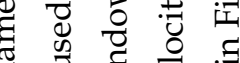

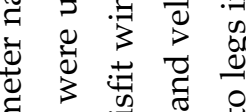

क

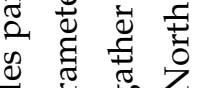

卷

웅ㅇㅇ

के

द्व $\hat{\jmath}_{0}$ के

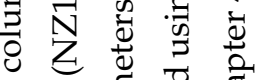

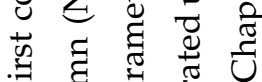

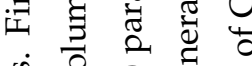

.

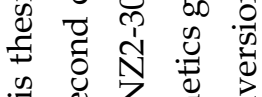

灵芯艺志

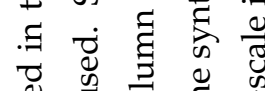

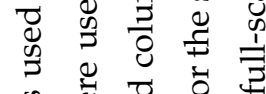

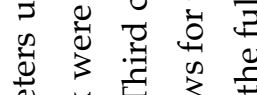

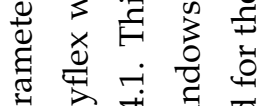

बै

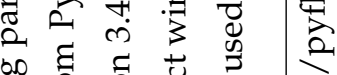

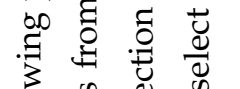




\section{Appendix B}

\section{Moment tensor preprocessing}

Moment tensors were gathered via the GeoNet GitHub data repository|1. Moment tensors are stored there in a comma separated values (csv) file, which among other parameters, lists moment tensor components in Mxx, Mxy, Mxz, Myy, Myz, Mzz. Units of moment tensors are given in 1E20 dyne cm, and are calculated following the methods of Ristau (2008).

SPECFEM expects moment tensors to be in units of RTP, that is Mrr, Mrt, Mrp, Mtt, Mrp, Mpp, and in units of dyne cm. To convert coordinates we follow Aki and Richards (2002):

- $\mathrm{Mrr}=\mathrm{Mzz}$

- $M t t=M x x$

- $\mathrm{Mpp}=$ Myy
- $\mathrm{Mrt}=\mathrm{Mxz}$

- $\operatorname{Mrp}=-1 \times \operatorname{Myz}$

- $\mathrm{Mtp}=-1 \times \mathrm{Mxy}$

Moment tensor components are multiplied by 1E-20 to convert to units of dyne $\mathrm{cm}$. Half durations that define the source time function were empirically derived following Dahlen and Tromp (1998) as:

$$
2.4 \mathrm{E}-6 \times M^{1 / 3}
$$

where $M$ is the seismic moment calculated from the components of the moment tensor. All synthetics were convolved with a Gaussian source time function with a corresponding half duration given by Equation B.1.

\footnotetext{
${ }^{1}$ https://github.com/GeoNet/data
} 


\section{Appendix C}

\section{Supplementary material for Chapter 2}

This appendix contains supplementary figures for Chapter2 Methods and data. 

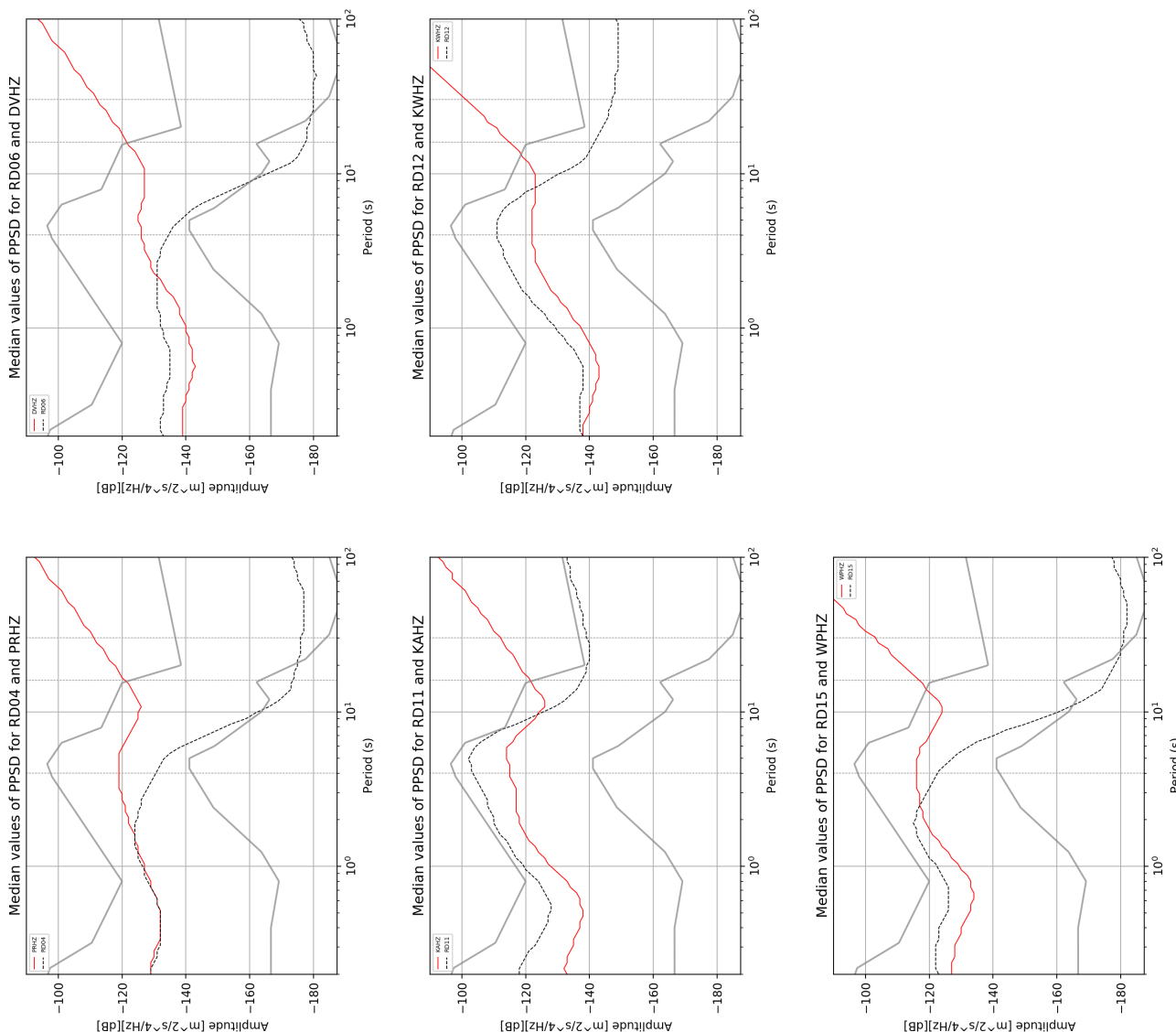

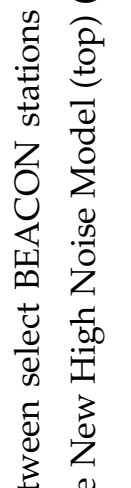
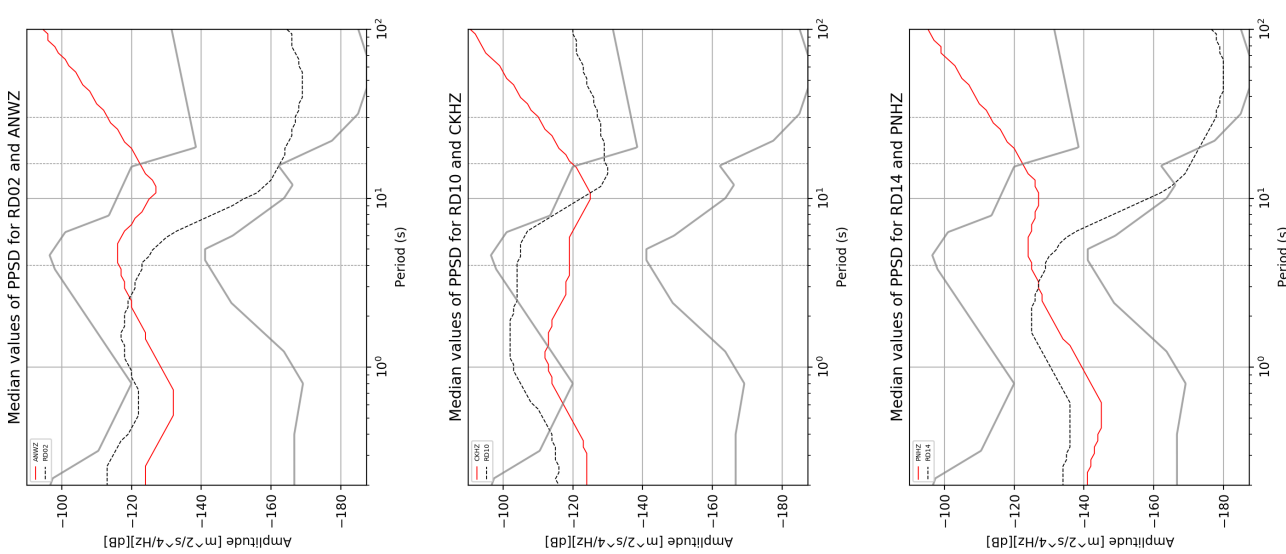

ڤั)

कि है

E द्व

索

ป

苞 $\sum_{0}^{0}$

范

की

¿ Z

30

范

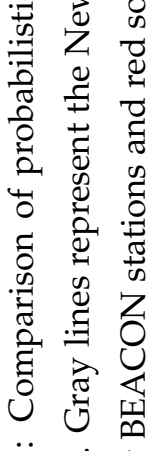

ن

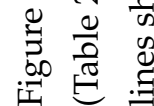
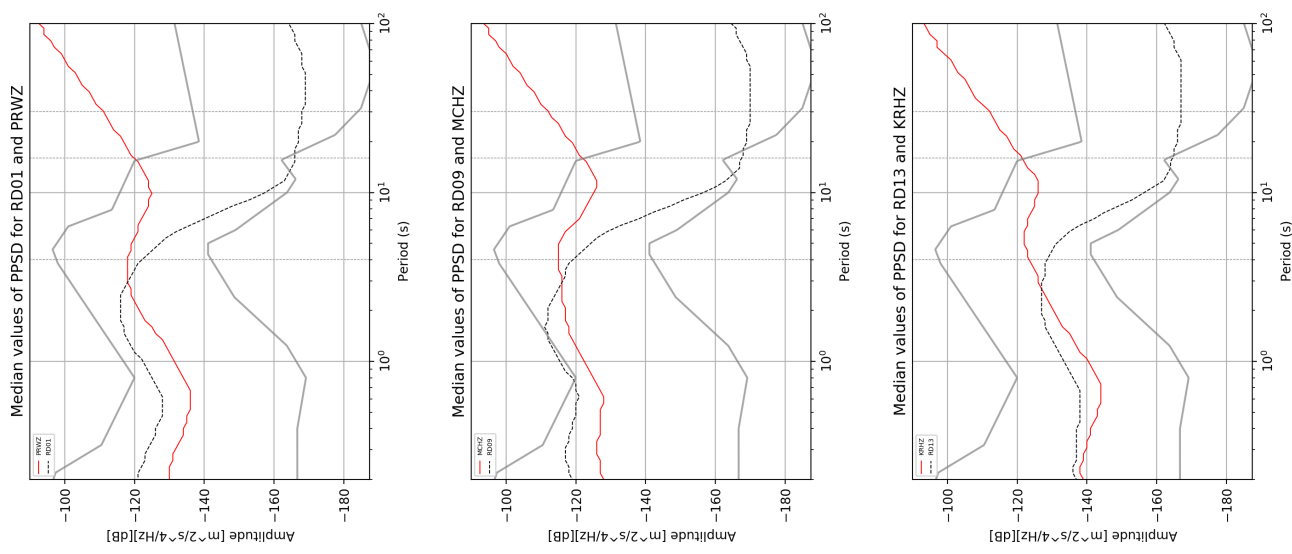


\section{Appendix D}

\section{Supplementary material for Chapter 3}

This appendix contains supplementary figures for Chapter 3 . An automated workflow for adjoint tomography. 


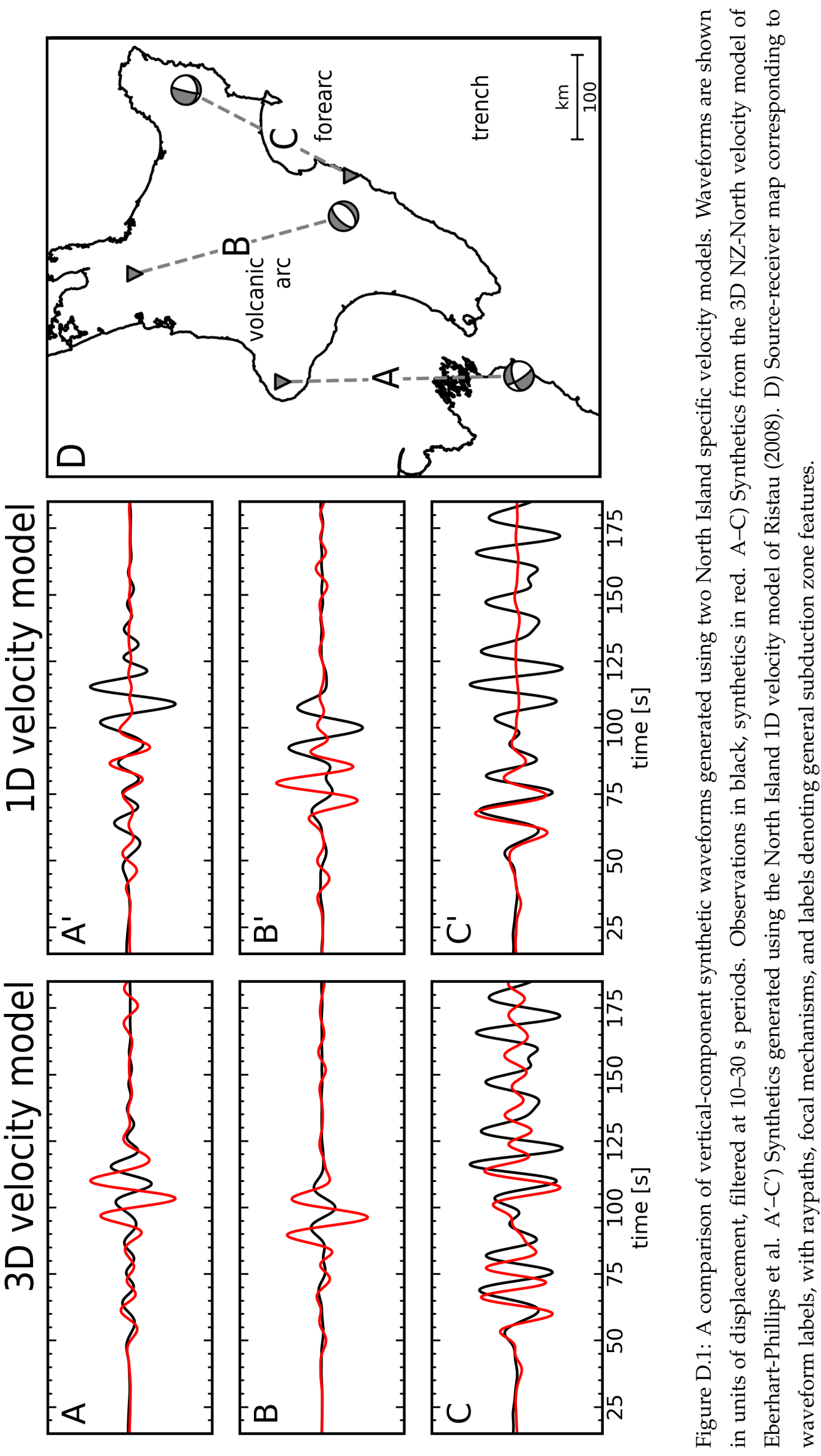



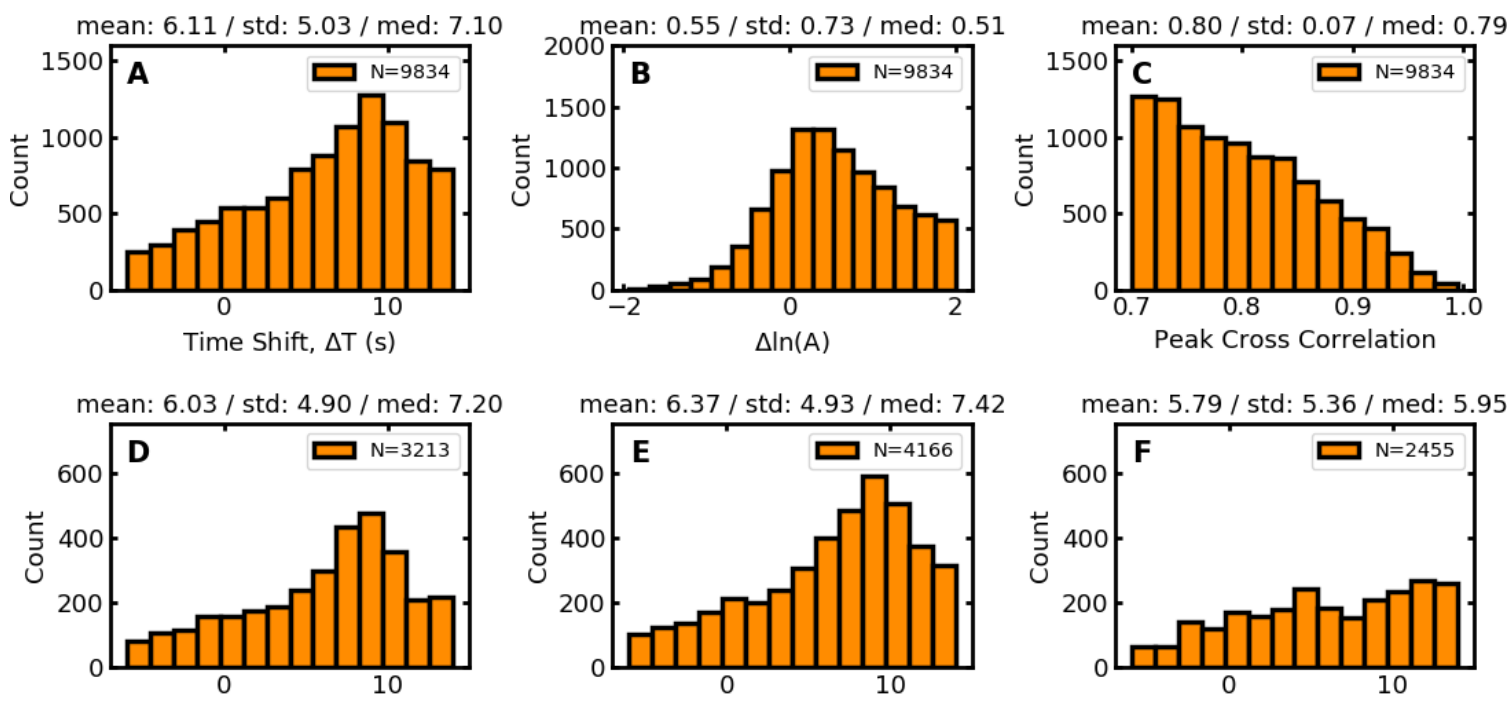

$\Delta \mathrm{T}(\mathrm{s})$, Vertical Component

$\Delta \mathrm{T}(\mathrm{s})$, Radial Component

$\Delta \mathrm{T}(\mathrm{s})$, Transverse Component
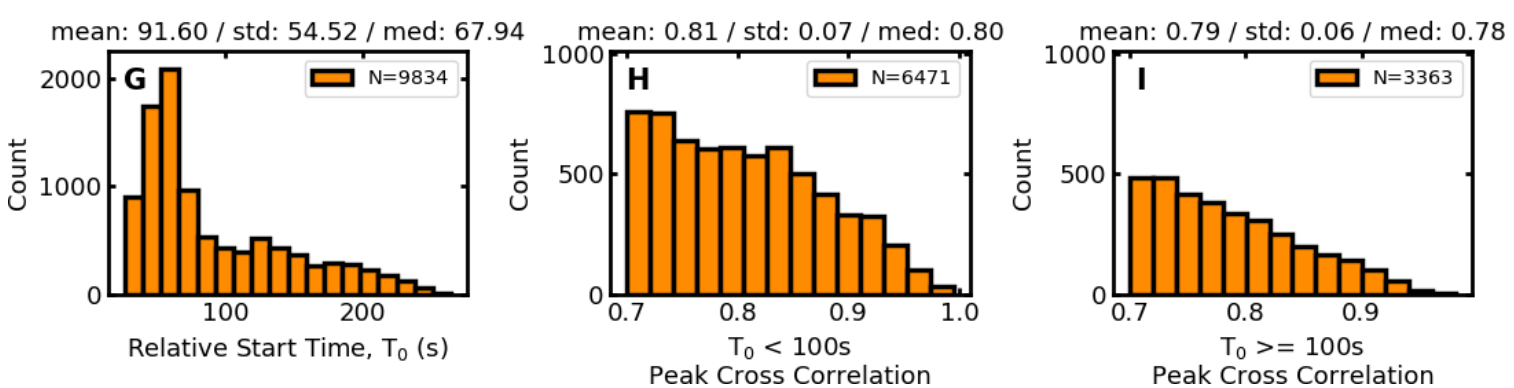

Figure D.2: Misfit histograms for forward simulations using the 1D North Island tomographic velocity model of Ristau (2008), in the same format as Figure 3.4. Measurements from 250 events recorded on three components of 45 broadband stations, with 9834 total measurements. Mean, median, and standard deviation given in the titles of each histogram, number of measurements for each provided in the legend. Windowing parameters are less conservative than those used to generate the histograms in Figure 3.4 A) Time shift corresponding to peak cross correlation between data and synthetics, in units of seconds. B) Data-synthetic amplitude difference $\Delta \ln (A)$ (Equation 3.1. C) Peak cross correlation value. D-F) Time shift for vertical, radial, and transverse component measurements. G) Measurement start time relative to event origin time. H) Peak cross correlations for measurements made before $100 \mathrm{~s}$ relative start time. I) Peak cross correlations for measurements made at or after $100 \mathrm{~s}$ relative start time. 

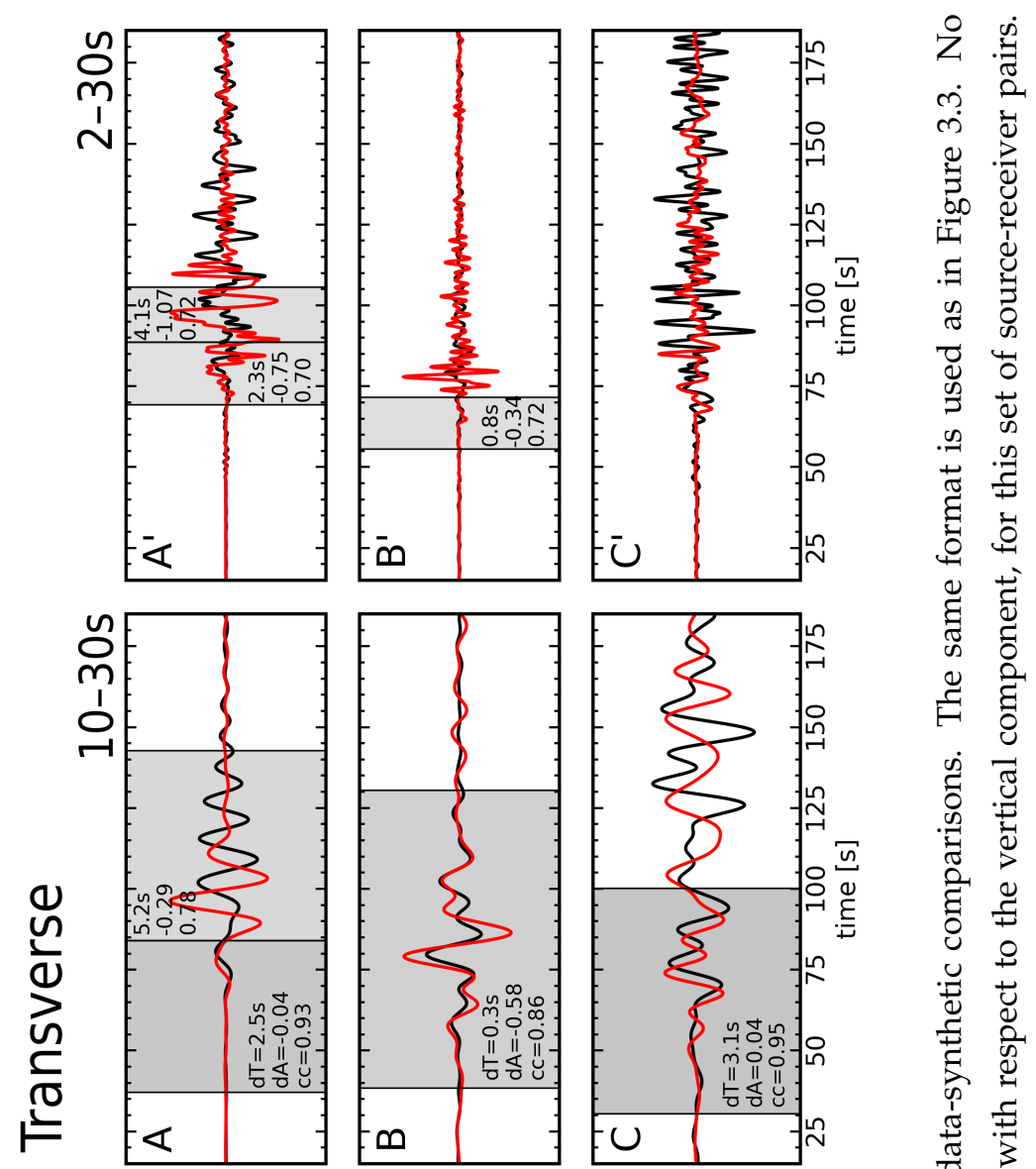

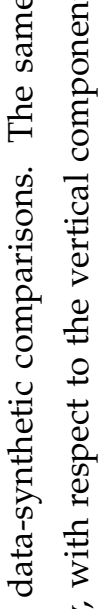
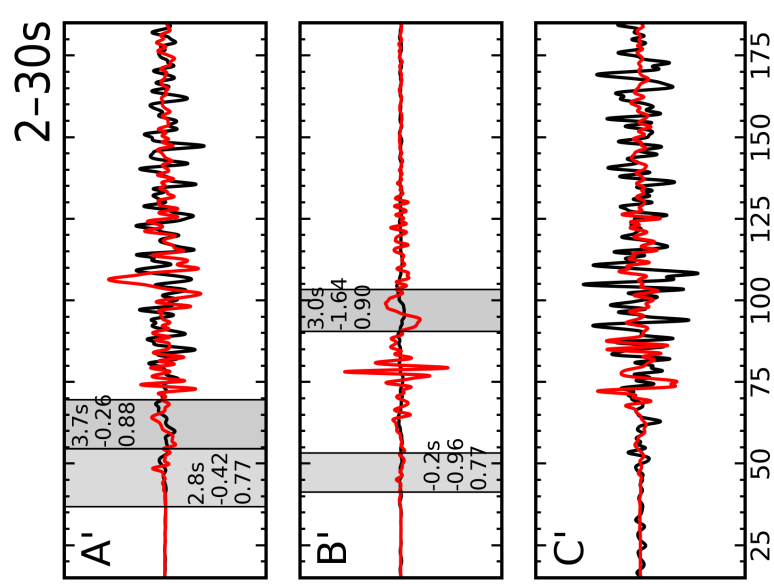

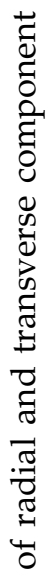
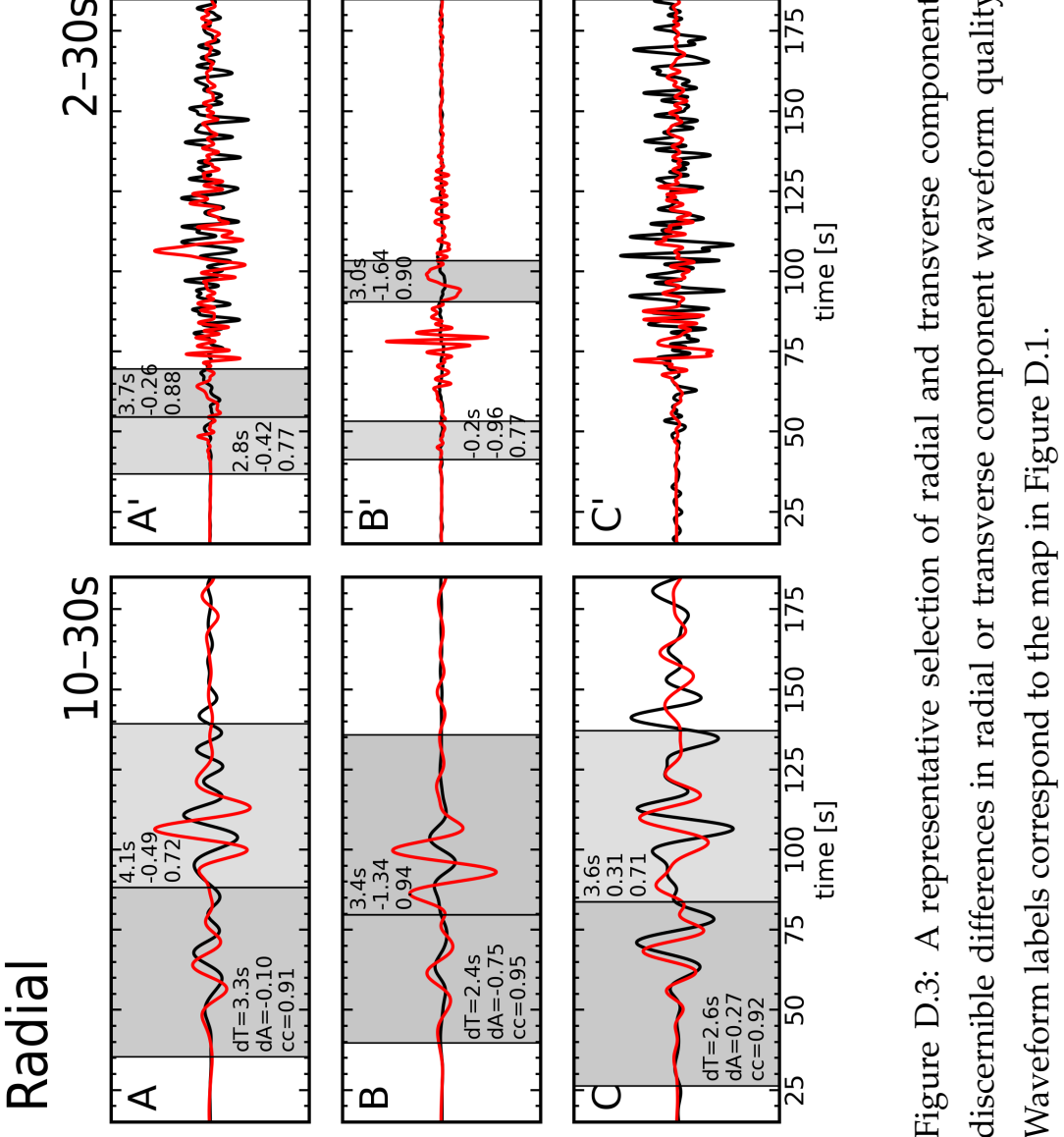
A) Inversion Preparation

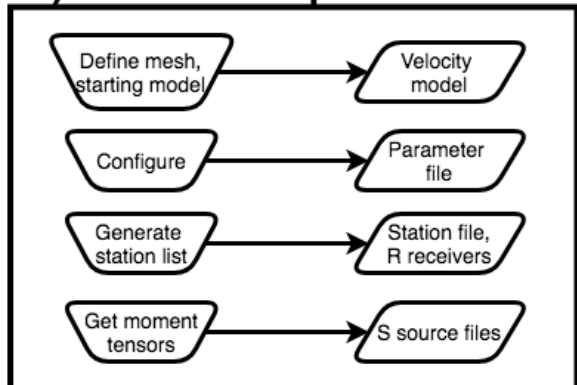

B) Seisflows Inversion

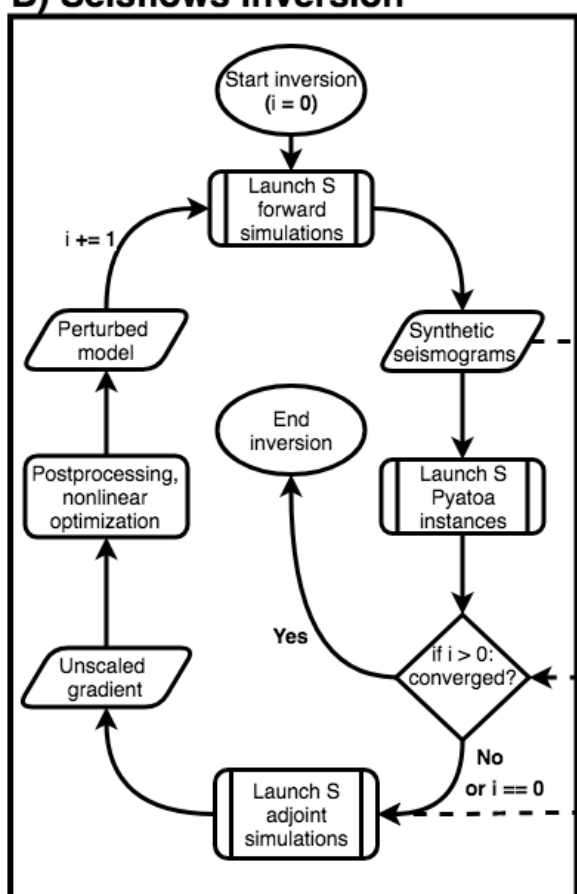

\section{D) Legend}

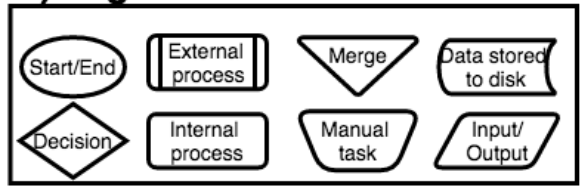

C) Pyatoa Misfit Quantification

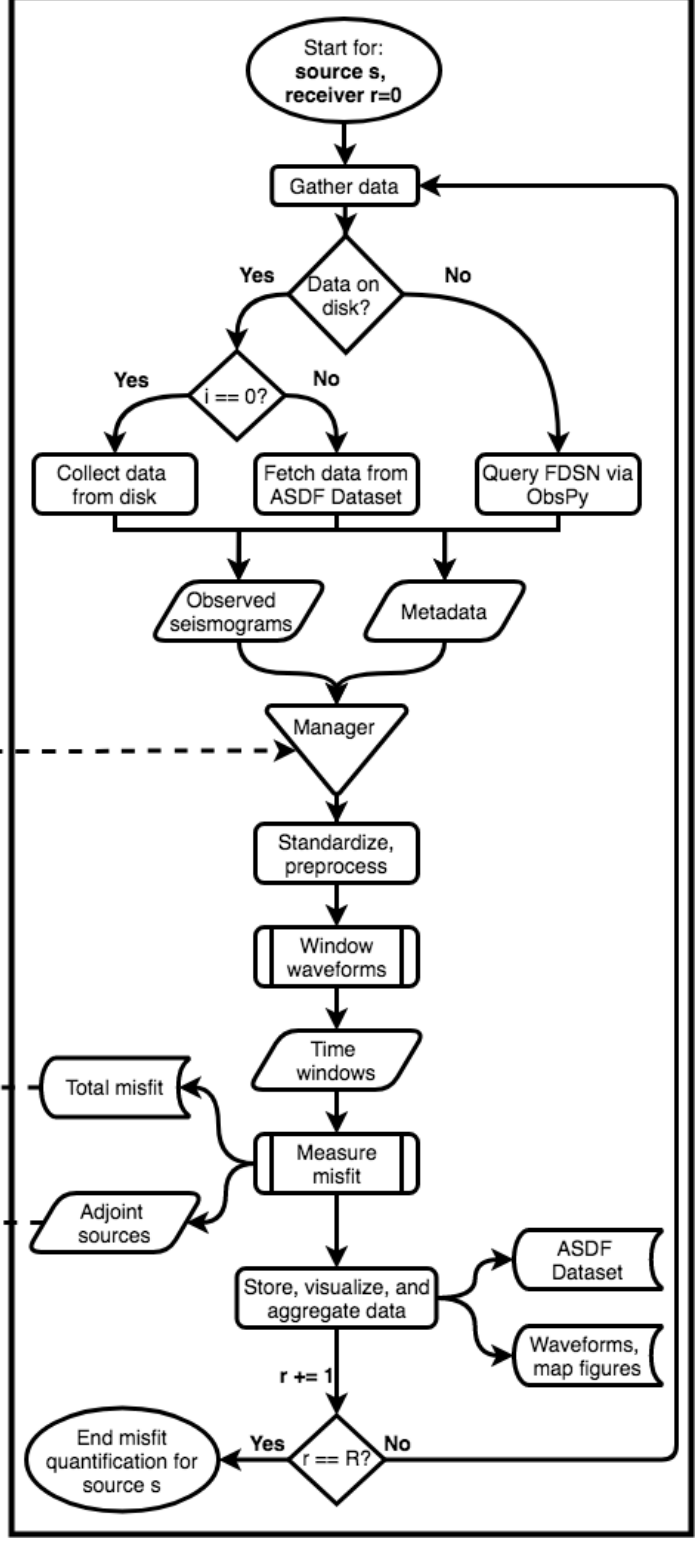

Figure D.4: A flowchart representing the inversion workflow. Dashed lines show interactions between Seisflows and Pyatoa via data stored to disk. A) A one-time manual preparation is required to generate the velocity model, define a single parameter file shared by Seisflows and Pyatoa, and create source and station files. B) A simplified Seisflows inversion, ignoring the complexities involved in, e.g., the optimization schema, workflow management, HPC job handling. Seisflows calls Pyatoa as a subroutine to calculate misfit and generate adjoint sources. C) A Pyatoa misfit quantification instance. Data is preferentially gathered from disk, but online web service queries are available via the International Federation of Digital Seismograph Networks (FDSN). Synthetic seismograms from SPECFEM are made accessible by Seisflows. A custom data structure (Manager) is responsible for collecting, processing, and measuring waveforms. Data is saved to disk using ASDF Datasets. Pyatoa provides additional auxiliary input files required for subsequent adjoint simulations launched by Seisflows. D) Legend describing the flowchart shapes. 

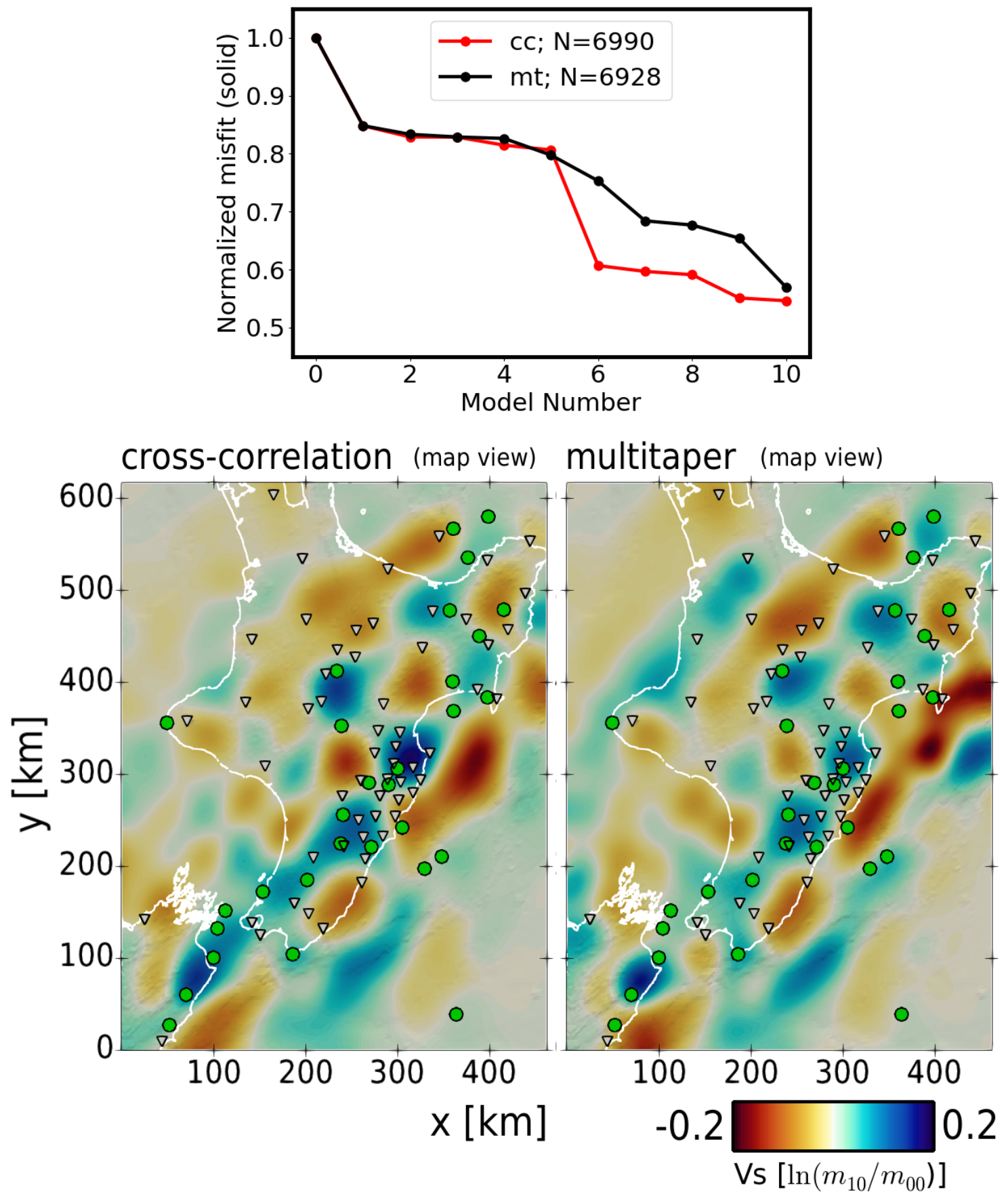

Figure D.5: Convergence behavior and map view of model 10 for synthetic inversions using the traveltime cross-correlation misfit (cc) and multitaper misfit (mt) functions, with a fixed set of time windows. Same as Figure 3.8 but with the same set of misfit windows, derived using the initial model, re-used throughout the inversion. Misfit normalized to start at 1 . As expected, misfit convergence behavior is shown to be more constrained than the open-window case. As with Figure 3.8, the final misfit is negligibly lower using the cross-correlation misfit, for this specific synthetic inversion scenario. 


\section{Appendix E}

\section{Supplementary material for Chapter 4}

This appendix contains supplementary figures for Chapter44: Adjoint tomography of the North Island of New Zealand. 

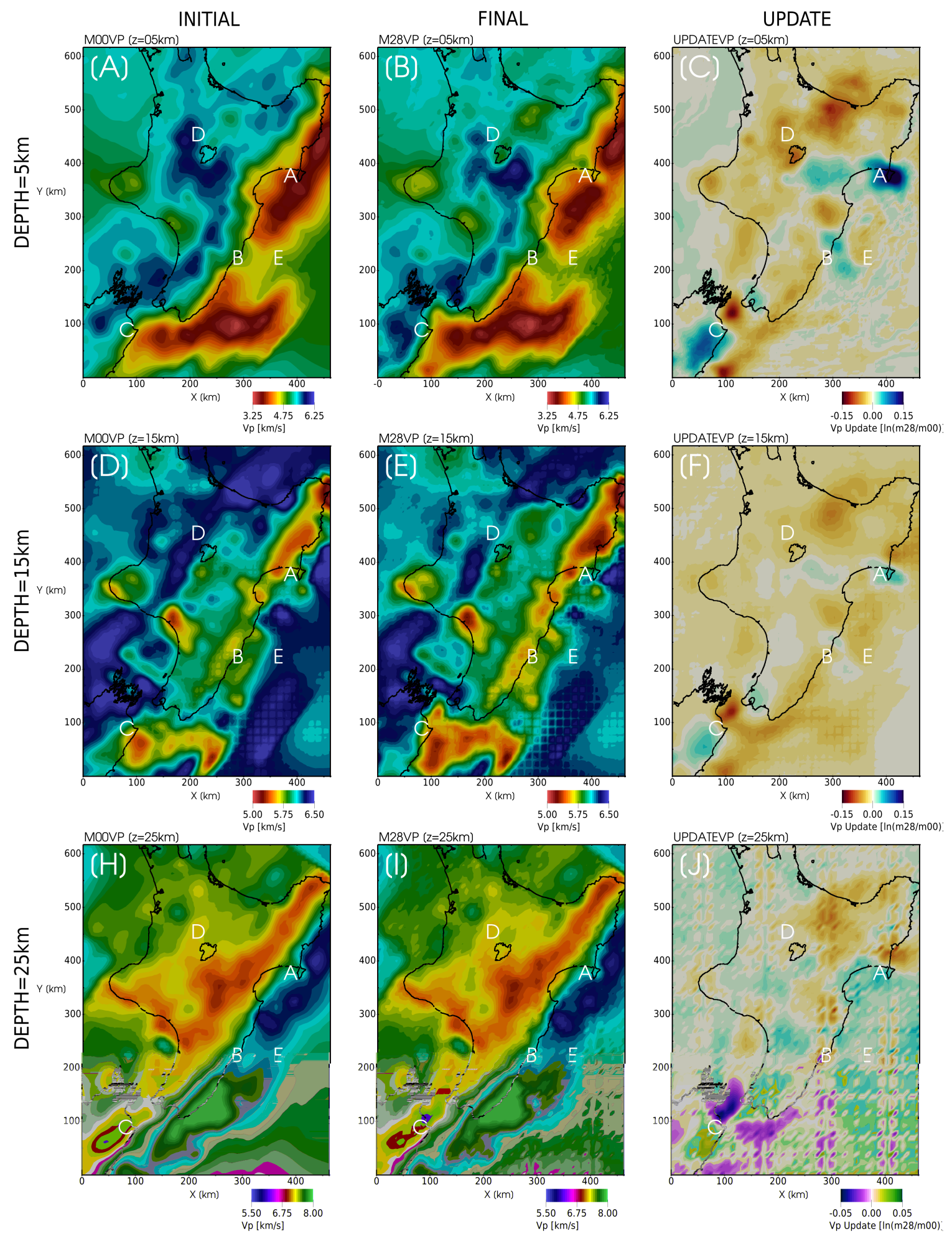

Figure E.1: Comparisons of M00 and M28 $\mathrm{V}_{p}$ velocity models at various depth slices. Columns represent initial model M00 (left), final model M28 (center), and net model update $\ln (\mathrm{M} 28 / \mathrm{M} 00)$ (right), while rows represent depth slices at $5 \mathrm{~km}$ (top), $15 \mathrm{~km}$ (middle), and $25 \mathrm{~km}$ (bottom). Annotated letters A-E relate to features discussed in Section 4.4. Note the different color scales between each row and columns. Numerical artefacts are visible in D, E, F, I, J, discussed in Section 4.3.3. 

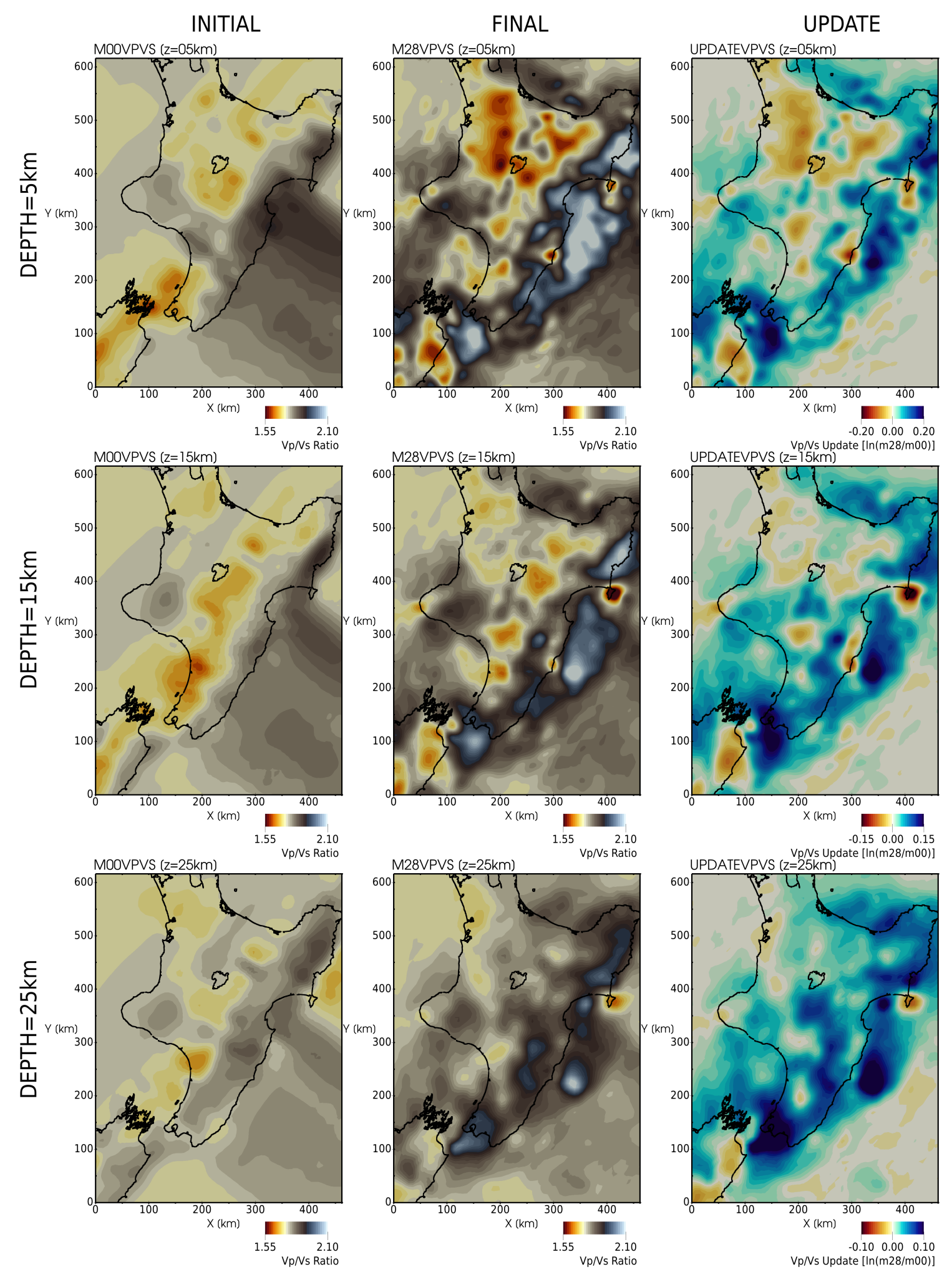

Figure E.2: Comparisons of M00 and $\mathrm{M} 28 \mathrm{~V}_{p} / \mathrm{V}_{s}$ velocity models at various depth slices. Columns represent initial model M00 (left), final model M28 (center), and net model update $\ln (\mathrm{M} 28 / \mathrm{M} 00)$ (right), while rows represent depth slices at $5 \mathrm{~km}$ (top), $15 \mathrm{~km}$ (middle), and $25 \mathrm{~km}$ (bottom). Annotated letters A-E relate to features discussed in Section 4.4 Note the differing color scales between net model update figures. 


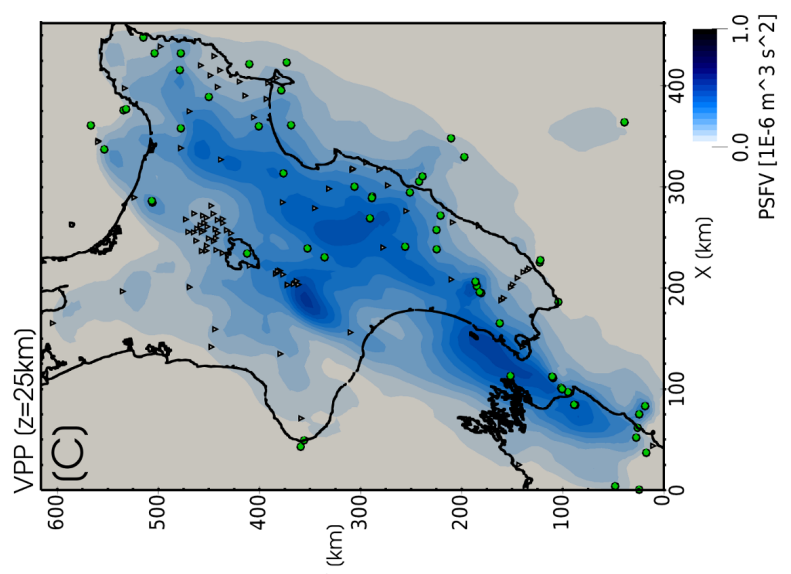

के

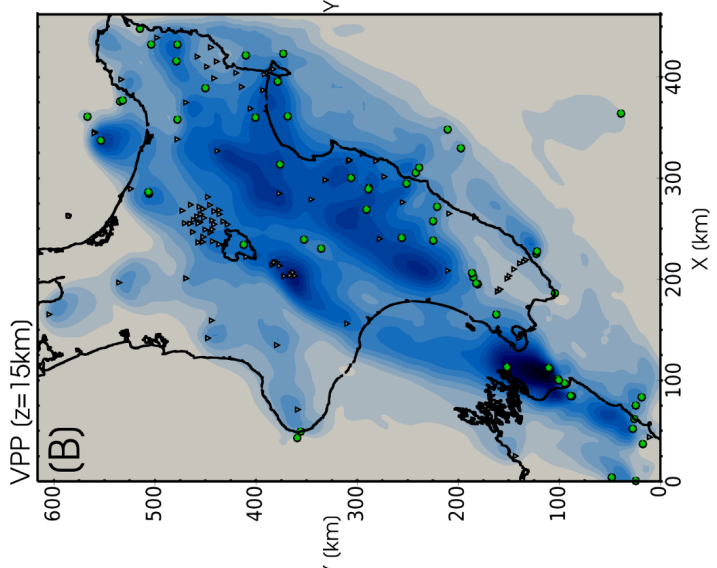

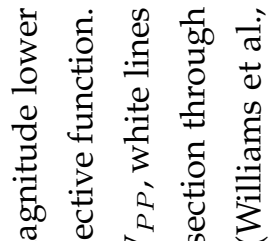

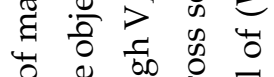

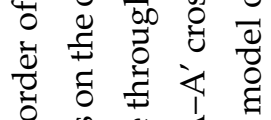

苞

.90 至

造

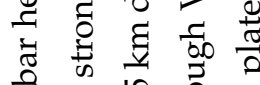

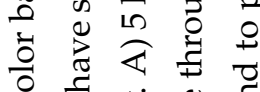

४े $\stackrel{0}{0}$

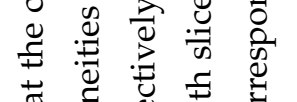

‡

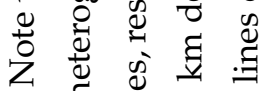

$\therefore$ व

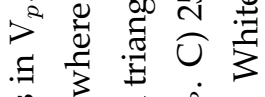

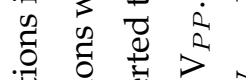

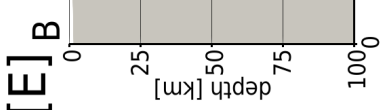

吾

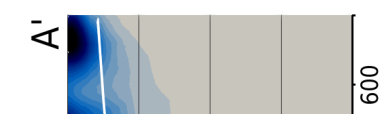

1응

过艺递

$-13 \%$

क के ट्ञ क्ज.

छ

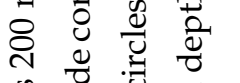

苛至苛

छे छे है

का तु

है छั

ฮ

के का चै

范

吾离离吉

。国.

艺

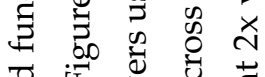

ฮ

के

吉合

ᄃ.

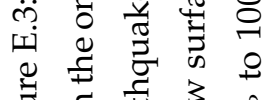

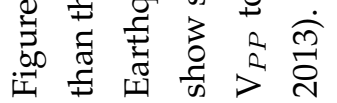




\section{Appendix F}

\section{Inversion parameter tables}

This appendix contains tables detailing the inversion and post-hoc analysis performed in Chapter 4 Table F.1 details the input and output parameters of the inversion in Chapter 4 , and is summarized visually in the convergence plot in Figure 4.3. Table F.2 and Table F.3 detail the earthquakes used in the inversion and post-hoc analysis, respectively. Table F.4 and Table F.5 respectively detail the permanent network and temporary network receivers used.

\section{Inversion parameters}

\begin{tabular}{|c|c|c|c|c|c|c|c|c|}
\hline Leg & Model & $\mathbf{T}_{\text {min-max }}[\mathbf{s}]$ & $\sigma_{h}[\mathbf{k m}]$ & $\sigma_{v}[\mathbf{k m}]$ & $\mathbf{N}_{\text {win }}$ & $\mathbf{T}_{\text {win }}[\mathbf{s}]$ & $\mathbf{F}(\mathbf{m})$ & Trials \\
\hline A & M00 & $15-30$ & 20 & 10 & 6302 & 499514 & 3.333 & - \\
\hline A & M01 & $15-30$ & 20 & 10 & 6302 & 499514 & 2.991 & 3 \\
\hline A & M02 & $15-30$ & 20 & 10 & 6302 & 499514 & 2.978 & 3 \\
\hline A & M03 & $15-30$ & 20 & 10 & 6302 & 499514 & 2.973 & 3 \\
\hline \hline B & M03 & $10-30$ & 20 & 10 & 7714 & 458422 & 1.870 & - \\
\hline B & M04 & $10-30$ & 20 & 10 & 7714 & 458422 & 1.777 & 3 \\
\hline B & M05 & $10-30$ & 20 & 10 & 7714 & 458422 & 1.769 & 3 \\
\hline B & M06 & $10-30$ & 20 & 10 & 7714 & 458422 & 1.747 & 1 \\
\hline B & M07 & $10-30$ & 20 & 10 & 7714 & 458422 & 1.708 & 1 \\
\hline B & M07 & $10-30$ & 20 & 10 & 7808 & 461039 & 1.687 & - \\
\hline B & M08 & $10-30$ & 20 & 10 & 7808 & 461039 & 1.665 & 4 \\
\hline B & M09 & $10-30$ & 20 & 10 & 7808 & 461039 & 1.639 & 1 \\
\hline B & M10 & $10-30$ & 20 & 10 & 7808 & 461039 & 1.617 & 2 \\
\hline
\end{tabular}




\begin{tabular}{|c|c|c|c|c|c|c|c|c|}
\hline C & M10 & $10-30$ & 10 & 7 & 7863 & 467184 & 1.638 & - \\
\hline C & M11 & $10-30$ & 10 & 7 & 7863 & 467184 & 1.635 & 2 \\
\hline C & M12 & $10-30$ & 10 & 7 & 7863 & 467184 & 1.634 & 2 \\
\hline C & M13 & $10-30$ & 10 & 7 & 7863 & 467184 & 1.626 & 4 \\
\hline \hline D & M13 & $8-30$ & 8.5 & 6 & 8899 & 406348 & 1.290 & - \\
\hline D & M14 & $8-30$ & 8.5 & 6 & 8899 & 406348 & 1.279 & 3 \\
\hline D & M15 & $8-30$ & 8.5 & 6 & 8899 & 408131 & 1.270 & 5 \\
\hline D & M15 & $8-30$ & 8.5 & 6 & 8928 & 408131 & 1.255 & - \\
\hline D & M16 & $8-30$ & 8.5 & 6 & 8928 & 408131 & 1.239 & 1 \\
\hline D & M17 & $8-30$ & 8.5 & 6 & 8928 & 408131 & 1.189 & 1 \\
\hline D & M18 & $8-30$ & 8.5 & 6 & 8928 & 408131 & 1.179 & 1 \\
\hline \hline E & M18 & $6-30$ & 8.5 & 6 & 11926 & 380158 & 1.506 & - \\
\hline E & M19 & $6-30$ & 8.5 & 6 & 11926 & 380158 & 1.498 & 4 \\
\hline E & M20 & $6-30$ & 8.5 & 6 & 11926 & 380158 & 1.488 & 1 \\
\hline E & M21 & $6-30$ & 8.5 & 6 & 11926 & 380158 & 1.485 & 1 \\
\hline E & M22 & $6-30$ & 8.5 & 6 & 11926 & 380158 & 1.481 & 2 \\
\hline E & M23 & $6-30$ & 8.5 & 6 & 11926 & 380158 & 1.476 & 3 \\
\hline E & M24 & $6-30$ & 8.5 & 6 & 11926 & 380158 & 1.462 & 6 \\
\hline E & M25 & $6-30$ & 8.5 & 6 & 11926 & 380158 & 1.462 & 1 \\
\hline \hline F & M25 & $4-30$ & 8 & 4 & 9256 & 216375 & 1.030 & - \\
\hline F & M26 & $4-30$ & 8 & 4 & 9256 & 216375 & 1.026 & 5 \\
\hline F & M27 & $4-30$ & 8 & 4 & 9256 & 216375 & 1.025 & 3 \\
\hline F & M28 & $4-30$ & 8 & 4 & 9256 & 216375 & 1.024 & 2 \\
\hline & & & & & & 6 & \\
\hline
\end{tabular}

Table F.1: Inversion parameters and results separated by inversion leg. Inversion legs correspond to colored lines in Figure 4.3 Waveform bandpass given in terms of period, units of seconds, as $T_{\min -\max }$. 3D smoothing Gaussian half-widths (Section 2.1.7) in the horizontal and vertical given as $\sigma_{h}$ and $\sigma_{v}$, respectively. Total number of time windows collected given as $\mathrm{N}_{\text {win }}$. Cumulative window length in seconds given in $\mathrm{T}_{\text {win }}$. Total misfit $\mathrm{F}(\mathrm{m})$ is defined by Equation 4.1. Trials column details the number of line search step counts required to find an acceptable step length to reduce misfit.

\section{Inversion events}

\begin{tabular}{|c|c|c|c|c|c|c|}
\hline & Event ID & Time [UTC] & $\mathbf{M}_{\mathrm{w}}$ & $\mathbf{Z}[\mathbf{k m}]$ & Lat $\left.^{\circ}{ }^{\circ}\right]$ & Lon $^{\circ}{ }^{\circ}$ \\
\hline 1 & 2228901 & $2004-05-11 T 23: 22: 03.427000 Z$ & 4.80 & 5.00 & -38.0250 & 178.1776 \\
\hline
\end{tabular}




\begin{tabular}{|c|c|c|c|c|c|c|}
\hline 2 & 2266782 & :58:06.069000Z & 4.90 & 5.00 & -38.0108 & 76.4969 \\
\hline 3 & 2266243 & 2004-07-18T04:22:22.727000Z & 5.40 & 5.00 & -38.0053 & 176.5141 \\
\hline 4 & 2986 & 05-01-18T08:36:04.241000Z & 5.20 & 18.76 & 1.4633 & 175.7893 \\
\hline 5 & 2354133 & 2005-01-20T18:56:31.429000Z & 5.30 & 30.84 & 1.0911 & 175.0526 \\
\hline 6 & 2359081 & 17:31:53.291000Z & 5.10 & 19.85 & -41.4572 & 175.7616 \\
\hline 7 & 2593170 & 2006-07-08T11:27:17.727000Z & 5.20 & 32.78 & -39.1795 & 176.8228 \\
\hline 8 & 2684211 & 2007-01-18T03:34:14.318000Z & 4.90 & 50.85 & -39.5410 & 175.8554 \\
\hline 9 & 2922302 & 2008-06-12T21:06:24.237000Z & 5.20 & 5.00 & 828 & 177.0946 \\
\hline 10 & 2974665 & 2008-09-26T07:23:02.845000Z & 5.00 & 23.89 & -42.3086 & 173.9235 \\
\hline 11 & 3337804 & 2010-07-11T13:23:4 & 5.10 & 33.00 & 589 & 177.3599 \\
\hline 12 & 3367989 & 2010-09-0 & 5.00 & 21.14 & 383 & 176.7229 \\
\hline 13 & 3369346 & 2010-09-09T04:18:48.330000Z & 4.90 & 15.71 & -40.4187 & 176.7836 \\
\hline 14 & 73 & $00 Z$ & 5.00 & 25.19 & 18 & 448 \\
\hline 15 & 3493233 & 2011-04-07 & 4.90 & 33.00 & 176 & 177.4264 \\
\hline 16 & 3540318 & 2011-07-04T08:36:51.677000Z & 4.80 & 12.00 & -40.7917 & 177.0092 \\
\hline 17 & 2656 & 2012 & 4.54 & 17.59 & 15 & 175.4123 \\
\hline 18 & 2012p560243 & 2012-07-26Т06:57:47. & 5.01 & 27.08 & 07 & 176.1585 \\
\hline 19 & $2012 p$ & 201 & 4.66 & 12.91 & 86 & 178 \\
\hline 20 & 2012p988084 & 2012-12-31T14:11:11.798788Z & 4.94 & 10.03 & -39.2865 & 173.6876 \\
\hline 21 & $2013 p$ & 2013 & 5.04 & 24.80 & 77 & 736 \\
\hline 22 & 2013 p507880 & 2013-07-07T21:58:46.744269Z & 4.94 & 30.38 & -39.8121 & 176.6668 \\
\hline 23 & 2 & Z & 5.69 & 2 & & 45 \\
\hline 24 & 2013p542711 & 2013-07-20T19:17:10.844582Z & 5.76 & 20.70 & -41.5537 & 174.3903 \\
\hline 25 & 2 & 2 & 5.18 & 8.86 & 4 & 93 \\
\hline 26 & 2013p613867 & 2013-08-16T03:09:07.725626Z & 5.62 & 7.46 & -41.7429 & 174.0611 \\
\hline 27 & 35 & $7 \mathrm{Z}$ & 5 & 14.96 & -41.6310 & 174.2540 \\
\hline 28 & 2013p617227 & 2013-08-17T08:58:40.318754Z & 5.49 & 15.07 & -41.7326 & 174.0642 \\
\hline 29 & 655 & 2014-03-31T01:01:19.4728 & 5.24 & 17.30 & 02 & 518 \\
\hline 30 & 2014 p240753 & $67114 Z$ & 4.55 & 13.14 & -3 & 176.5349 \\
\hline 31 & 2014 p715167 & 2014 & 5.45 & 25.39 & 04 & 175.9324 \\
\hline 32 & 2014p952799 & $.482070 Z$ & 4.91 & 33.06 & 520 & 177.9926 \\
\hline 33 & $2015 p 2$ & 2015 & 4.56 & 9.62 & -37 & 544 \\
\hline 34 & 2015 p768477 & $17692 Z$ & 5.69 & 23.28 & 780 & 176.3257 \\
\hline 35 & $2015 p 822263$ & 2015-11-01T05:42:11.671776Z & 5.38 & 54.92 & -39.3893 & 175.9597 \\
\hline 36 & 2016p105478 & 2016-02-09T00:39:00.115717Z & 5.74 & 48.12 & -42.0682 & 173.0748 \\
\hline
\end{tabular}




\begin{tabular}{|c|c|c|c|c|c|c|}
\hline 37 & 2016p198476 & 2016-03-14T10:28:04.208382Z & 5.03 & 8.83 & -42.2804 & 173.0207 \\
\hline 38 & 2016 p275188 & 2016-04-11T19:41:06.568548Z & 5.20 & 23.86 & -40.8929 & 175.4943 \\
\hline 39 & 2016p356297 & 2016-05-11T19:55:18.243942Z & 5.16 & 27.26 & -40.9171 & 175.4226 \\
\hline 40 & $2016 p 842451$ & 2016-11-07T16:59:07.167808Z & 5.14 & 13.51 & -39.3187 & 173.7597 \\
\hline 41 & 2016p858279 & 2016-11-13T13:31:27.507278Z & 5.98 & 13.95 & -42.2741 & 173.6455 \\
\hline 42 & 2016p858951 & 2016-11-13T19:28:56.934526Z & 5.58 & 5.00 & -42.3637 & 174.0193 \\
\hline 43 & 2016p862339 & 2016-11-15T01:34:45.265970Z & 5.81 & 5.81 & -41.6817 & 174.2169 \\
\hline 44 & 2016p881118 & 2016-11-22T00:19:42.525939Z & 5.54 & 29.11 & -40.6735 & 177.2288 \\
\hline 45 & 2017p015402 & 2017-01-06T16:46:12.15 & 4.83 & 27.98 & -37.7519 & 177.5345 \\
\hline 46 & 2017p084950 & 2017-02-01T10:21:29.759031Z & 5.08 & 21.13 & -42.2903 & 173.7621 \\
\hline 47 & 2017p087060 & 2017-02-02T05:05:39.319521Z & 4.82 & 27.48 & -37.7757 & 177.5468 \\
\hline 48 & 2017p266099 & 2017-04-09T10:57:59.815172Z & 4.75 & 14.25 & -41.6171 & 175.2894 \\
\hline 49 & 2017p292246 & 2017-04-19T03:08:42.632596Z & 5.08 & 38.04 & -38.5123 & 177.6910 \\
\hline 50 & 2017p795065 & 2017-10-22T04:10:59.674111Z & 5.58 & 8.27 & -42.3581 & 173.4589 \\
\hline 51 & 2017p819775 & 2017-10-31T07:36:07.022652Z & 4.75 & 11.62 & -37.4626 & 177.3622 \\
\hline 52 & 2017p852531 & 2017-11-12T10:28:56.348882Z & 4.81 & 35.70 & -41.1744 & 174.4269 \\
\hline 53 & 2018p130600 & 2018-02-18T07:43:48.127644Z & 5.15 & 20.59 & -39.9489 & 176.2995 \\
\hline 54 & $2018 \mathrm{p} 465580$ & 2018-06-22T06:21:00.801931Z & 4.90 & 16.73 & -40.3064 & 176.5974 \\
\hline 55 & 2019 p248841 & 2019-04-03Т01:42:43.082882Z & 4.60 & 13.39 & 031 & 178.0928 \\
\hline 56 & 2019p304574 & 2019-04-23T16:37:10.033368Z & 4.79 & 24.61 & -40.2598 & 175.9666 \\
\hline 57 & 2019p665658 & 2019-09-04T07:02:52.385251Z & 5.01 & 5.00 & -38.8513 & 175.9097 \\
\hline 58 & 2019 p738432 & 2019-10-01T05:16:41.168796Z & 5.65 & 22.00 & -39.1577 & 177.7724 \\
\hline 59 & 2019 p754447 & 2019-10-07Т03:29:48.367981Z & 5.28 & 38.14 & -38.2642 & 177.3336 \\
\hline 60 & 2019p927023 & 2019-12-09T23:58:01.907909Z & 5.38 & 29.01 & -38.8703 & 178.0682 \\
\hline
\end{tabular}

Table F.2: 60 earthquakes used in the inversion in Chapter 4. Events are detailed by their GeoNet Event ID, origin time in Coordinated Universal Time (UTC), moment magnitude $\left(\mathrm{M}_{\mathrm{w}}\right)$, depth in $\mathrm{km}(\mathrm{Z})$, and latitude and longitude values in degrees. Events sorted by origin time from earliest to latest, also reflected in the GeoNet Event Ids. Note the change of event ID format between 2011 and 2012. Earthquake locations and moment tensors shown in Figure 3.1

\section{Post-hoc events}

\begin{tabular}{|c|c|c|c|c|c|c|}
\hline & Event ID & Time [UTC] & $\mathbf{M}_{\mathrm{w}}$ & $\mathbf{Z}[\mathbf{k m}]$ & Lat $\left.^{\circ}{ }^{\circ}\right]$ & Lon $\left.^{\circ}{ }^{\circ}\right]$ \\
\hline 1 & 2366369 & $2005-02-17 T 21: 31: 10.175000 Z$ & 4.60 & 27.90 & -39.9041 & 174.6025 \\
\hline
\end{tabular}




\begin{tabular}{|c|c|c|c|c|c|c|}
\hline 2 & 3205715 & $09-12-0$ & 4.60 & 48.91 & -40.5 & 74.8535 \\
\hline 3 & 3361067 & 010-08-24T22:17:36.813000Z & 4.60 & 29.91 & 0.1067 & 174.8938 \\
\hline 4 & 74473 & 1-03-06Т03:43:33.682000Z & 4.70 & 9.74 & 2.3411 & 73.8629 \\
\hline 5 & 3570483 & 2011-08-29T19:11:53.399000Z & 4.50 & 3.00 & 7.8343 & 176.6283 \\
\hline 6 & 2013p128381 & 12:01:29.473847Z & 4.69 & 22.93 & .7720 & 73.8836 \\
\hline 7 & $2013 p 432496$ & 2013-06-10T00:3 & 4.66 & 22.03 & .1436 & 77.4066 \\
\hline 8 & 2013 p543121 & 2013-07-20T22:55:16.892551Z & 4.96 & 19.65 & 1.5623 & 174.3844 \\
\hline 9 & 2013 p544960 & 2013-07-21T15:15:11.707128Z & 4.90 & 14.49 & 00 & 174.3100 \\
\hline 10 & 2013p545809 & 2013-07-21T22:47:24.323905Z & 4.96 & 15.08 & 6115 & 174.2554 \\
\hline 11 & 2013p547469 & $2013-07-22$ & 4.82 & 19.88 & 619 & 174.3444 \\
\hline 12 & $2013 \mathrm{p}$ & $6534 Z$ & 4.76 & 17.07 & 582 & 866 \\
\hline 13 & 2013p614294 & 2013-08-16T06:55:58.920101Z & 5.47 & 17.07 & -41.6776 & 174.2765 \\
\hline 14 & 201 & $64 Z$ & .19 & 17.07 & 24 & 306 \\
\hline 15 & 2013p616691 & 2013 & 5.01 & 8.63 & 55 & 174.0037 \\
\hline 16 & 18032 & 2013-08-17 & 4.99 & 16.95 & 91 & 174 \\
\hline 17 & 20131 & $73 Z$ & 5.02 & 13.09 & 27 & 174.4300 \\
\hline 18 & 2013p739681 & 2013-10-01T16:22:32.953294Z & 4.67 & 21.64 & 05 & 174.3953 \\
\hline 19 & 2014 & $44 Z$ & 61 & 17.25 & 59 & 868 \\
\hline 20 & 2014 p753812 & 2014-10-06T21:51:08.633838Z & 4.57 & 12.01 & -40.4242 & 176.8101 \\
\hline 21 & 2015 & $1 Z$ & 35 & 18.01 & 24 & 05 \\
\hline 22 & 2016 p858508 & 2016-11-13T15:33:04.701947Z & 5.53 & 7.18 & -41.7899 & 174.2350 \\
\hline 23 & 3 & Z & 4.70 & 10.02 & & 00 \\
\hline 24 & 2016p858876 & 2016-11-13T18:49:25.101598Z & 4.71 & 5.78 & -41.9706 & 174.0321 \\
\hline 25 & 2 & 2 & 5.36 & 3 & 5 & 54 \\
\hline 26 & 2016p859378 & 2016-11-13Т23:17:33.343459Z & 5.17 & 9.82 & -42.1030 & 173.8491 \\
\hline 27 & 29 & 3 & 5.14 & $50-30+2$ & 0 & 24 \\
\hline 28 & 2016 p860215 & 2016-11-14T06:43:00.476414Z & 5.58 & 13.15 & -41.7669 & 174.2633 \\
\hline 29 & 2016 & $07.21 \cdot 048897$ & 5.75 & 11.48 & 67 & 872 \\
\hline 30 & 2016p860567 & $300 Z$ & 0.01 & 9.00 & 15 & 173.7742 \\
\hline 31 & 2016p861632 & 201 & 5.14 & 5.00 & -42.2380 & 173.6978 \\
\hline 32 & 2016 p862742 & 5T05:09:27.646158Z & 5.27 & 11.16 & 33 & 173.6487 \\
\hline 33 & 2016 & 2016- & 4.74 & 8.82 & -41 & 174 \\
\hline 34 & 2016p864842 & 2016 & 4.60 & 9.63 & 084 & 174.0157 \\
\hline 35 & 2016 p865557 & 2016-11-16T06:09:39.435496Z & 4.81 & 5.00 & -41.7057 & 174.4227 \\
\hline 36 & 2016 p866561 & 2016-11-16T15:03:49.905573Z & 4.78 & 5.00 & -42.4707 & 173.3717 \\
\hline
\end{tabular}




\begin{tabular}{|c|c|c|c|c|c|c|}
\hline 37 & 2016p871629 & 2016-11-18T12:03:56.860852Z & 4.60 & 5.00 & -41.9942 & 173.5889 \\
\hline 38 & $2016 p 880389$ & 2016-11-21T17:51:37.034407Z & 4.98 & 5.00 & -42.1544 & 173.5819 \\
\hline 39 & 2016p913880 & 2016-12-04T03:15:47.108522Z & 5.52 & 10.66 & -41.6903 & 174.2357 \\
\hline 40 & 2016p969664 & 2016-12-24T18:37:22.245801Z & 4.68 & 15.08 & -41.6369 & 174.2701 \\
\hline 41 & 2016p976987 & $11: 38: 46.406522 Z$ & 4.73 & 13.20 & -42.3863 & 173.7083 \\
\hline 42 & 2016p981371 & 2016-12-29T02:34:33.905128Z & 5.56 & 16.02 & -41.5491 & 174.4619 \\
\hline 43 & 2017p012082 & 2017-01-05T11:17:34.879638Z & 5.41 & 14.37 & -41.8075 & 174.0366 \\
\hline 44 & 2017p260291 & 2017-04-07Т07:23:23.803522Z & 4.58 & 10.07 & -41.9823 & 173.8672 \\
\hline 45 & 2017p346659 & 2017-05-09T06:19:58.362606Z & 4.70 & 31.02 & -37.7877 & 177.5927 \\
\hline 46 & 2017p381325 & 2017-05-22T02:10:04.883509Z & 4.81 & 5.41 & -42.2078 & 173.6417 \\
\hline 47 & 2017p861155 & 2017-11-15T15:03:33 & 4.68 & 10.97 & -41.5617 & 174.3072 \\
\hline 48 & 2018 p354699 & 2018-05-12T05:43:45.711461Z & 4.64 & 10.42 & -42.2866 & 173.8335 \\
\hline 49 & 2019 p248841 & 2019-04-03Т01:42:43.082882Z & 4.61 & 13.40 & -39.2032 & 178.0928 \\
\hline 50 & 2019p304574 & 2019-04-23T16:37:10.033368Z & 4.79 & 24.61 & -40.2598 & 175.9667 \\
\hline 51 & 2019p665661 & 2019-09-04T07:04:34.465987Z & 4.51 & 5.00 & -38.8577 & 175.9264 \\
\hline 52 & 2019 p738432 & 2019-10-01T05:16:41.168796Z & 5.65 & 22.00 & -39.1578 & 177.7724 \\
\hline 53 & 2019 p754447 & 2019-10-07Т03:29:48.367981Z & 5.28 & 38.14 & -38.2643 & 177.3337 \\
\hline 54 & 2019p927023 & 2019-12-09T23:58:01.907909Z & 5.39 & 29.01 & -38.8703 & 178.0683 \\
\hline 55 & 2019p938537 & 2019-12-14T06:12:36.879424Z & 4.87 & 44.81 & -35 & 176.1367 \\
\hline 56 & 2020p391429 & 2020-05-24T19:53:33.278747Z & 5.85 & 36.52 & -40.4461 & 174.9778 \\
\hline 57 & $2020 p 408400$ & 2020-05-31T02:35:49.974273Z & 4.95 & 5.00 & -38.7061 & 174.0467 \\
\hline 58 & $2020 p 450618$ & 2020-06-15T17:29:46.143605Z & 4.94 & 28.76 & -37.8167 & 177.5600 \\
\hline 59 & 2020 p730156 & 2020-09-27T03:47:23.861676Z & 5.15 & 22.60 & -39.1916 & 174.9135 \\
\hline 60 & 2021p064325 & 2021-01-24T19:12:34.624295Z & 4.91 & 5.00 & -38.0862 & 176.4939 \\
\hline
\end{tabular}

Table F.3: 60 earthquakes used in the post-hoc analysis in Chapter 4. Events are detailed by their GeoNet Event ID, origin time in Coordinated Universal Time (UTC), moment magnitude $\left(\mathrm{M}_{\mathrm{w}}\right)$, depth in $\mathrm{km}(\mathrm{Z})$, and latitude and longitude values in degrees. Events sorted by origin time from earliest to latest, also reflected in the GeoNet Event Ids. Note the change of event ID format between 2011 and 2012.

\section{Inversion receivers}




\begin{tabular}{|c|c|c|c|}
\hline Net & Station & Lat $\left[^{\circ}\right]^{\text {L }}$ & Lo $^{\circ}$ ] \\
\hline NZ & BFZ & -40.6796 & 176.2462 \\
\hline NZ & BHW & -41.4082 & 174.8711 \\
\hline NZ & BKZ & -39.1656 & 176.4925 \\
\hline NZ & COVZ & -39.1999 & 175.5424 \\
\hline NZ & ETVZ & -39.1357 & 175.7106 \\
\hline NZ & FWVZ & -39.2549 & 175.5529 \\
\hline NZ & HAZ & -37.7561 & 177.7826 \\
\hline NZ & HIZ & -38.5129 & 174.8556 \\
\hline NZ & KHEZ & -39.2942 & 174.0145 \\
\hline NZ & KHZ & -42.4159 & 173.5389 \\
\hline NZ & KNZ & -39.0217 & 177.6736 \\
\hline NZ & MAVZ & -39.2679 & 175.5616 \\
\hline NZ & MRZ & -40.6605 & 175.5785 \\
\hline NZ & MWZ & -38.3340 & 177.5277 \\
\hline NZ & MXZ & -37.5622 & 178.3066 \\
\hline NZ & NNZ & -41.2171 & 173.3794 \\
\hline NZ & NTVZ & -39.0984 & 175.6759 \\
\hline NZ & OPRZ & -37.8443 & 176.5549 \\
\hline NZ & OTVZ & -39.1631 & 175.6650 \\
\hline
\end{tabular}

\begin{tabular}{|c|c|c|c|}
\hline Net & Station & Lat $\left[^{\circ}\right]^{\text {Lon }}{ }^{\circ}{ }^{\circ}$ \\
\hline NZ & PUZ & -38.0715 & 178.2572 \\
\hline NZ & RATZ & -38.8664 & 175.7721 \\
\hline NZ & RTZ & -38.6154 & 176.9805 \\
\hline NZ & TLZ & -38.3294 & 175.5380 \\
\hline NZ & TMVZ & -39.1156 & 175.7040 \\
\hline NZ & TOZ & -37.7309 & 175.5018 \\
\hline NZ & TRVZ & -39.2988 & 175.5478 \\
\hline NZ & TSZ & -40.0585 & 175.9611 \\
\hline NZ & URZ & -38.2592 & 177.1108 \\
\hline NZ & VRZ & -39.1243 & 174.7584 \\
\hline NZ & WAZ & -39.7546 & 174.9855 \\
\hline NZ & WEL & -41.2840 & 174.7681 \\
\hline NZ & WHVZ & -39.2825 & 175.5886 \\
\hline NZ & WIZ & -37.5245 & 177.1893 \\
\hline NZ & WSRZ & -37.5181 & 177.1778 \\
\hline NZ & PWZ & -40.0296 & 176.8619 \\
\hline NZ & WPVZ & -39.2040 & 175.5459 \\
\hline NZ & MKAZ & -37.1041 & 175.1611 \\
\hline
\end{tabular}

Table F.4: 38 GeoNet permanent network broadband stations used in the inversion of Chapter 4 given as network and station code, and latitude and longitude values in degrees. Station locations shown on a map in Figure 2.6 


\begin{tabular}{|c|c|c|c|}
\hline Net & Station & Lat ${ }^{\circ}{ }^{\circ}$ & Lon $\left[^{\circ}\right]^{]}$ \\
\hline ZX & GA01 & -39.0331 & 177.8549 \\
\hline ZX & GA02 & -38.7793 & 177.8695 \\
\hline ZX & GA04 & -38.5529 & 178.1548 \\
\hline ZX & GA05 & -38.5844 & 177.8048 \\
\hline ZX & GA06 & -39.1110 & 177.9161 \\
\hline ZX & GA07 & -38.4322 & 178.0478 \\
\hline ZX & GA08 & -38.8312 & 177.7095 \\
\hline ZX & GA09 & -38.5194 & 177.9363 \\
\hline ZX & GA10 & -38.9087 & 177.4627 \\
\hline ZX & GA03 & -38.6059 & 177.9959 \\
\hline X2 & LE4 & -41.3579 & 175.6919 \\
\hline X2 & T004 & -41.3403 & 175.6688 \\
\hline X2 & T010 & -41.2520 & 175.5825 \\
\hline X2 & T014 & -41.2075 & 175.5063 \\
\hline X2 & T016 & -41.1893 & 175.4737 \\
\hline X2 & T020 & -41.1251 & 175.3497 \\
\hline X2 & LTN6 & -41.1033 & 175.3238 \\
\hline Z8 & HD02 & -38.6275 & 175.9196 \\
\hline Z8 & HD04 & -38.4930 & 176.2204 \\
\hline Z8 & HD05 & -38.4663 & 176.2627 \\
\hline Z8 & HD06 & -38.3932 & 176.0619 \\
\hline Z8 & HD08 & -38.6300 & 176.3063 \\
\hline Z8 & HD09 & -38.6675 & 176.1798 \\
\hline Z8 & HD10 & -38.5482 & 176.3669 \\
\hline Z8 & HD11 & -38.6320 & 176.2606 \\
\hline
\end{tabular}

\begin{tabular}{|c|c|c|c|}
\hline Net & Station & Lat $^{\circ}{ }^{\circ}$ & Lon $\left[^{\circ}\right]$ \\
\hline Z8 & HD12 & -38.3711 & 176.1570 \\
\hline Z8 & HD13 & -38.4546 & 176.3458 \\
\hline Z8 & HD14 & -38.4594 & 176.1714 \\
\hline Z8 & HD15 & -38.4872 & 176.0043 \\
\hline Z8 & HD16 & -38.4408 & 175.9444 \\
\hline Z8 & HD17 & -38.5483 & 175.0556 \\
\hline Z8 & HD18 & -38.5283 & 176.4573 \\
\hline Z8 & HD19 & -38.3762 & 176.3696 \\
\hline Z8 & HD20 & -38.5842 & 176.1467 \\
\hline Z8 & HD21 & -38.5052 & 176.0877 \\
\hline Z8 & HD22 & -38.5666 & 176.1907 \\
\hline Z8 & HD24 & -38.5671 & 176.0895 \\
\hline Z8 & HD25 & -38.5855 & 176.2945 \\
\hline Z8 & HD26 & -38.5696 & 175.9547 \\
\hline Z8 & HD27 & -38.4324 & 176.2374 \\
\hline Z8 & HD28 & -38.4424 & 176.1580 \\
\hline Z8 & HD29 & -38.4012 & 176.1991 \\
\hline Z8 & HD31 & -38.3019 & 176.3053 \\
\hline XX & RD10 & -39.6466 & 177.0765 \\
\hline XX & RD11 & -39.7873 & 176.8624 \\
\hline XX & RD12 & -39.4252 & 176.4228 \\
\hline XX & RD16 & -40.1053 & 176.6804 \\
\hline XX & RD17 & -39.9086 & 176.9561 \\
\hline XX & RD03 & -40.2656 & 176.3828 \\
\hline XX & RD19 & -39.5796 & 176.6449 \\
\hline
\end{tabular}

Table F.5: 50 temporary network broadband stations used in the inversion of Chapter 4 . given as network and station code, and latitude and longitude values in degrees. Network codes refer to data provided by Stephen Bannister (ZX, Z8), the SAHKE deployment (X2) (Henrys et al. 2013), and the BEACON deployment (XX) (Section 2.3. Network and station locations shown on a map in Figure 2.6 


\section{Bibliography}

Afanasiev, M., Boehm, C., van Driel, M., Krischer, L., Rietmann, M., May, D. A., Knepley, M. G., and Fichtner, A. (2019). Modular and flexible spectral-element waveform modelling in two and three dimensions. Geophysical Journal International, 216(3):1675-1692.

Aki, K. and Lee, W. (1976). Determination of three-dimensional velocity anomalies under a seismic array using first $\mathrm{P}$ arrival times from local earthquakes: 1 . A homogeneous initial model. Journal of Geophysical Research, 81(23):4381-4399.

Aki, K. and Richards, P. G. (2002). Quantitative seismology.

Aki Christofferson, A. and Husebye, E. (1977). Three-dimensional seismic structure of the lithosphere. Journal of Geophysical Research, 82:277-296.

Audet, P., Bostock, M. G., Christensen, N. I., and Peacock, S. M. (2009). Seismic evidence for overpressured subducted oceanic crust and megathrust fault sealing. Nature, 457(7225):76-78.

Bannister, S., Fry, B., Reyners, M., Ristau, J., and Zhang, H. (2011). Fine-scale relocation of aftershocks of the 22 February Mw 6.2 Christchurch earthquake using double-difference tomography. Seismological Research Letters, 82(6):839_ 845.

Bao, X. and Shen, Y. (2016). Assessing waveform predictions of recent threedimensional velocity models of the Tibetan Plateau. Journal of Geophysical Research: Solid Earth, 121:2521-2538.

Barker, D. H., Sutherland, R., Henrys, S., and Bannister, S. (2009). Geometry of the Hikurangi subduction thrust and upper plate, North Island, New Zealand. Geochemistry, Geophysics, Geosystems, 10(2). 
Barker, S. J., Rowe, M. C., Wilson, C. J., Gamble, J. A., Rooyakkers, S. M., Wysoczanski, R. J., Illsley-Kemp, F., and Kenworthy, C. C. (2020). What lies beneath? Reconstructing the primitive magmas fueling voluminous silicic volcanism using olivine-hosted melt inclusions. Geology, 48(5):504-508.

Barnes, P. M. and Audru, J.-C. (1999). Quaternary faulting in the offshore Flaxbourne and Wairarapa basins, southern Cook Strait, New Zealand. New Zealand Journal of Geology and Geophysics, 42(3):349-367.

Barnes, P. M., de Lépinay, B. M., Collot, J.-Y., Delteil, J., and Audru, J.-C. (1998). Strain partitioning in the transition area between oblique subduction and continental collision, Hikurangi margin, New Zealand. Tectonics, 17(4):534-557.

Barnes, P. M., Lamarche, G., Bialas, J., Henrys, S., Pecher, I., Netzeband, G. L., Greinert, J., Mountjoy, J. J., Pedley, K., and Crutchley, G. (2010). Tectonic and geological framework for gas hydrates and cold seeps on the Hikurangi subduction margin, New Zealand. Marine Geology, 272(1-4):26-48.

Barnes, P. M., Nicol, A., and Harrison, T. (2002). Late Cenozoic evolution and earthquake potential of an active listric thrust complex above the Hikurangi subduction zone, New Zealand. Geological Society of America Bulletin, 114(11):1379_ 1405.

Bassett, D., Sutherland, R., and Henrys, S. (2014). Slow wavespeeds and fluid overpressure in a region of shallow geodetic locking and slow slip, Hikurangi subduction margin, New Zealand. Earth and Planetary Science Letters, 389:1-13.

Beanland, S. (1995). The North Island Dextral Fault Belt, Hikurangi Subduction Margin, New Zealand. PhD thesis, Victoria University of Wellington.

Beanland, S. and Haines, J. (1998). The kinematics of active deformation in the North Island, New Zealand, determined from geological strain rates. New Zealand Journal of Geology and Geophysics, 41(4):311-323.

Becker, J., Sandwell, D., Smith, W., Braud, J., Binder, B., Depner, J., Fabre, D., Factor, J., Ingalls, S., Kim, S., et al. (2009). Global bathymetry and elevation data at 30 arc seconds resolution: SRTM30_PLUS. Marine Geodesy, 32(4):355-371. 
Behr, Y., Townend, J., Bannister, S., and Savage, M. K. (2011). Crustal shear wave tomography of the Taupō Volcanic Zone, New Zealand, via ambient noise correlation between multiple three-component networks. Geochemistry, Geophysics, Geosystems, 12(3).

Bell, R., Holden, C., Power, W., Wang, X., and Downes, G. (2014). Hikurangi margin tsunami earthquake generated by slow seismic rupture over a subducted seamount. Earth and Planetary Science Letters, 397:1-9.

Bell, R., Sutherland, R., Barker, D. H., Henrys, S., Bannister, S., Wallace, L., and Beavan, J. (2010). Seismic reflection character of the Hikurangi subduction interface, New Zealand, in the region of repeated Gisborne slow slip events. Geophysical Journal International, 180(1):34-48.

Berryman, K., Beanland, S., Cooper, A., Cutten, H., and Norris, R. (1992). The Alpine Fault, New Zealand: variation in Quaternary structural style and geomorphic expression.

Beyreuther, M., Barsch, R., Krischer, L., Megies, T., Behr, Y., and Wassermann, J. (2010). ObsPy: A python toolbox for seismology. Seismological Research Letters, 81(3):530-533.

Bijwaard, H. and Spakman, W. (1999). Tomographic evidence for a narrow whole mantle plume below Iceland. Earth and Planetary Science Letters, 166(3-4):121126.

Bijwaard, H., Spakman, W., and Engdahl, E. R. (1998). Closing the gap between regional and global travel time tomography. Journal of Geophysical Research: Solid Earth, 103(B12):30055-30078.

Bland, K. J., Uruski, C. I., and Isaac, M. J. (2015). Pegasus Basin, eastern New Zealand: A stratigraphic record of subsidence and subduction, ancient and modern. New Zealand Journal of Geology and Geophysics, 58(4):319-343.

Boese, C., Townend, J., Smith, E., and Stern, T. (2012). Microseismicity and stress in the vicinity of the Alpine Fault, central Southern Alps, New Zealand. Journal of Geophysical Research: Solid Earth, 117(B2). 
Borisov, D., Modrak, R., Gao, F., and Tromp, J. (2018). 3D elastic full-waveform inversion of surface waves in the presence of irregular topography using an envelope-based misfit function. Geophysics, 83(1):R1-R11.

Bormann, P. (2012). New manual of seismological observatory practice (NMSOP2), IASPEI, GFZ German Research Centre for Geosciences, Potsdam.

Bozdağ, E., Peter, D., Lefebvre, M., Komatitsch, D., Tromp, J., Hill, J., Podhorszki, N., and Pugmire, D. (2016). Global adjoint tomography: first-generation model. Geophysical Journal International, 207(3):1739-1766.

Bozdağ, E., Trampert, J., and Tromp, J. (2011). Misfit functions for full waveform inversion based on instantaneous phase and envelope measurements. Geophysical Journal International, 185(2):845-870.

Bradley, B. A., Bae, S. E., Polak, V., Lee, R. L., Thomson, E. M., and Tarbali, K. (2017). Ground motion simulations of great earthquakes on the Alpine Fault: effect of hypocentre location and comparison with empirical modelling. New Zealand Journal of Geology and Geophysics, 60(3):188-198.

Bullen, K. E. (1950). An Earth model based on a compressibility-pressure hypothesis. Geophysical Journal International, 6:50-59.

Carter, L., Lewis, K. B., and Davey, F. (1988). Faults in Cook Strait and their bearing on the structure of central New Zealand. New Zealand Journal of Geology and Geophysics, 31(4):431-436.

Carter, R. M. and Naish, T. R. (1998). A review of Wanganui Basin, New Zealand: global reference section for shallow marine, Plio-Pleistocene (2.5-0 Ma) cyclostratigraphy. Sedimentary Geology, 122(1-4):37-52.

Chambefort, I., Lewis, B., Wilson, C., Rae, A., Coutts, C., Bignall, G., and Ireland, T. (2014). Stratigraphy and structure of the Ngatamariki geothermal system from new zircon $\mathrm{U}-\mathrm{Pb}$ geochronology: Implications for Taupo Volcanic Zone evolution. Journal of Volcanology and Geothermal Research, 274:51-70.

Chamberlain, C. J., Frank, W., Lanza, F., Townend, J., and Warren-Smith, E. (2021). Illuminating the pre-, co-, and post-seismic phases of the 2016 M7. 8 Kaikourra earthquake with 10 years of seismicity. Journal of Geophysical Research: Solid Earth, page e2021JB022304. 
Chamberlain, C. J., Shelly, D. R., Townend, J., and Stern, T. A. (2014). Lowfrequency earthquakes reveal punctuated slow slip on the deep extent of the Alpine fault, New Zealand. Geochemistry, Geophysics, Geosystems, 15(7):29842999.

Chen, M., Niu, F., Liu, Q., Tromp, J., and Zheng, X. (2015). Multiparameter adjoint tomography of the crust and upper mantle beneath East Asia: 1. Model construction and comparisons. Journal of Geophysical Research: Solid Earth, 120(3):17621786.

Chen, P., Zhao, L., and Jordan, T. H. (2007a). Full 3D tomography for the crustal structure of the Los Angeles region. Bulletin of the Seismological Society of America, 97(4):1094-1120.

Chen, P., Zhao, L., and Jordan, T. H. (2007b). Full 3D tomography for the crustal structure of the Los Angeles region. Bulletin of the Seismological Society of America, 97(4):1094-1120.

Chow, B., Kaneko, Y., Tape, C., Modrak, R., and Townend, J. (2020). An automated workflow for adjoint tomography - waveform misfits and synthetic inversions for the North Island, New Zealand. Geophysical Journal International, 223(3):14611480 .

Clark, K., Howarth, J., Litchfield, N., Cochran, U., Turnbull, J., Dowling, L., Howell, A., Berryman, K., and Wolfe, F. (2019). Geological evidence for past large earthquakes and tsunamis along the Hikurangi subduction margin, New Zealand. Marine Geology, 412:139-172.

Cochran, U., Berryman, K., Zachariasen, J., Mildenhall, D., Hayward, B., Southall, K., Hollis, C., Barker, P., Wallace, L., Alloway, B., et al. (2006). Paleoecological insights into subduction zone earthquake occurrence, eastern North Island, New Zealand. GSA Bulletin, 118(9-10):1051-1074.

Cole, J., Darby, D., and Stern, T. (1995). Taupo Volcanic Zone and Central Volcanic Region backarc structures of North Island, New Zealand. In Backarc Basins, pages 1-28. Springer.

Collot, J.-Y., Delteil, J., Lewis, K. B., Davy, B., Lamarche, G., Audru, J.-C., Barnes, P., Chanier, F., Chaumillon, E., Lallemand, S., et al. (1996). From oblique subduc- 
tion to intra-continental transpression: Structures of the southern KermadecHikurangi margin from multibeam bathymetry, side-scan sonar and seismic reflection. Marine Geophysical Researches, 18(2):357-381.

Collot, J.-Y., Lewis, K., Lamarche, G., and Lallemand, S. (2001). The giant Ruatoria debris avalanche on the northern Hikurangi margin, New Zealand: Result of oblique seamount subduction. Journal of Geophysical Research: Solid Earth, 106(B9):19271-19297.

Courant, R., Friedrichs, K., and Lewy, H. (1967). On the partial difference equations of mathematical physics. IBM journal of Research and Development, 11(2):215-234.

Cummins, P. R., Baba, T., Kodaira, S., and Kaneda, Y. (2002). The 1946 Nankai earthquake and segmentation of the Nankai Trough. Physics of the Earth and Planetary Interiors, 132(1-3):75-87.

Dahlen, F. and Baig, A. M. (2002). Fréchet kernels for body-wave amplitudes. Geophysical Journal International, 150(2):440-466.

Dahlen, F., Hung, S.-H., and Nolet, G. (2000). Fréchet kernels for finite-frequency traveltimes-I. Theory. Geophysical Journal International, 141(1):157-174.

Dahlen, F. and Nolet, G. (2005). Comment on 'On sensitivity kernels for 'waveequation' transmission tomography'by de Hoop and van der Hilst. Geophysical Journal International, 163(3):949-951.

Dahlen, F. A. and Tromp, J. (1998). Theoretical global seismology. Princeton university press.

Darby, D. and Beavan, J. (2001). Evidence from GPS measurements for contemporary interplate coupling on the southern Hikurangi subduction thrust and for partitioning of strain in the upper plate. Journal of Geophysical Research: Solid Earth, 106(B12):30881-30891.

Darby, D. J., Hodgkinson, K. M., and Blick, G. H. (2000). Geodetic measurement of deformation in the Taupo Volcanic Zone, New Zealand: the north Taupo network revisited. New Zealand Journal of Geology and Geophysics, 43(2):157-170. 
Davy, B., Hoernle, K., and Werner, R. (2008). Hikurangi Plateau: Crustal structure, rifted formation, and Gondwana subduction history. Geochemistry, Geophysics, Geosystems, 9(7).

Davy, B. and Wood, R. (1994). Gravity and magnetic modelling of the Hikurangi Plateau. Marine Geology, 118(1-2):139-151.

De Hoop, M. V. and van Der Hilst, R. D. (2005). On sensitivity kernels for 'waveequation' transmission tomography. Geophysical Journal International, 160(2):621633.

DeMets, C., Gordon, R. G., Argus, D. F., and Stein, S. (1994). Effect of recent revisions to the geomagnetic reversal time scale on estimates of current plate motions. Geophysical research letters, 21(20):2191-2194.

Descartes, R. and Laurence, J. (1631). 1631. Discourse on method and meditations (trans.).

Dixon, T. H., Jiang, Y., Malservisi, R., McCaffrey, R., Voss, N., Protti, M., and Gonzalez, V. (2014). Earthquake and tsunami forecasts: Relation of slow slip events to subsequent earthquake rupture. Proceedings of the National Academy of Sciences, 111(48):17039-17044.

Dominguez, S., Lallemand, S., Malavieille, J., and von Huene, R. (1998). Upper plate deformation associated with seamount subduction. Tectonophysics, 293(34):207-224.

Dominguez, S., Malavieille, J., and Lallemand, S. E. (2000). Deformation of accretionary wedges in response to seamount subduction: Insights from sandbox experiments. Tectonics, 19(1):182-196.

Douglas, A., Beavan, J., Wallace, L., and Townend, J. (2005). Slow slip on the northern Hikurangi subduction interface, New Zealand. Geophysical Research Letters, 32(16).

Dragert, H., Wang, K., and James, T. S. (2001). A silent slip event on the deeper Cascadia subduction interface. Science, 292(5521):1525-1528.

Dreger, D. S. (2003). TDMT_INV: Time domain seismic moment tensor inversion. In International Geophysics, volume 81, page 1627. Elsevier. 
Dziewonski, A. M., Hager, B. H., and O'Connell, R. J. (1977). Large-scale heterogeneities in the lower mantle. Journal of Geophysical Research, 82(2):239-255.

Eberhart-Phillips, D. and Bannister, S. (2010). 3-D imaging of Marlborough, New Zealand, subducted plate and strike-slip fault systems. Geophysical Journal International, 182(1):73-96.

Eberhart-Phillips, D. and Bannister, S. (2015). 3-D imaging of the northern Hikurangi subduction zone, New Zealand: variations in subducted sediment, slab fluids and slow slip. Geophysical Journal International, 201(2):838-855.

Eberhart-Phillips, D., Bannister, S., and Ellis, S. (2014). Imaging P and S attenuation in the termination region of the Hikurangi subduction zone, New Zealand. Geophysical Journal International, 198(1):516-536.

Eberhart-Phillips, D., Bannister, S., and Reyners, M. (2017). Deciphering the 3-D distribution of fluid along the shallow Hikurangi subduction zone using P-and S-wave attenuation. Geophysical Journal International, 211(2):1032-1045.

Eberhart-Phillips, D., Bannister, S., and Reyners, M. (2020a). Attenuation in the mantle wedge beneath super-volcanoes of the Taupō Volcanic Zone, New Zealand. Geophysical Journal International, 220(1):703-723.

Eberhart-Phillips, D., Bannister, S., Reyners, M., and Henrys, S. (2020b). New Zealand Wide model 2.2 seismic velocity and Qs and Qp models for New Zealand. Zenodo [Data set], http://doi.org/10.5281/zenodo.3779523.

Eberhart-Phillips, D. and Fry, B. (2017). A new scheme for joint surface wave and earthquake travel-time inversion and resulting 3-D velocity model for the western North Island, New Zealand. Physics of the Earth and Planetary Interiors, 269:98-111.

Eberhart-Phillips, D. and Fry, B. (2018). Joint local earthquake and teleseismic inversion for 3-D velocity and Q in New Zealand. Physics of the Earth and Planetary Interiors, 283:48-66.

Eberhart-Phillips, D., Han, D.-H., and Zoback, M. D. (1989). Empirical relationships among seismic velocity, effective pressure, porosity, and clay content in sandstone. Geophysics, 54(1):82-89. 
Eberhart-Phillips, D. and Reyners, M. (1997). Continental subduction and threedimensional crustal structure: The northern South Island, New Zealand. Journal of Geophysical Research: Solid Earth, 102(B6):11843-11861.

Eberhart-Phillips, D. and Reyners, M. (1999). Plate interface properties in the northeast Hikurangi subduction zone, New Zealand, from converted seismic waves. Geophysical Research Letters, 26(16):2565-2568.

Eberhart-Phillips, D. and Reyners, M. (2012). Imaging the Hikurangi Plate interface region, with improved local-earthquake tomography. Geophysical Journal International, 190(2):1221-1242.

Eberhart-Phillips, D., Reyners, M., and Bannister, S. (2015). A 3D QP attenuation model for all of New Zealand. Seismological Research Letters, 86(6):1655-1663.

Eberhart-Phillips, D., Reyners, M., Bannister, S., Chadwick, M., and Ellis, S. (2010). Establishing a versatile 3-D seismic velocity model for New Zealand. Seismological Research Letters, 81(6):992-1000.

Eberhart-Phillips, D., Reyners, M., Chadwick, M., and Chiu, J.-M. (2005). Crustal heterogeneity and subduction processes: 3-D Vp, Vp/Vs and $\mathrm{Q}$ in the southern North Island, New Zealand. Geophysical Journal International, 162(1):270-288.

Eberhart-Phillips, D., Reyners, M., Upton, P., and Gubbins, D. (2018). Insights into the structure and tectonic history of the southern South Island, New Zealand, from the 3-D distribution of P-and S-wave attenuation. Geophysical Journal International, 214(2):1481-1505.

Edbrooke, S., Heron, D., Forsyth, P., and Jongens, R. (2015). Geological map of New Zealand 1:1 000 000. GNS Science Geological Map 2.

Ekstrom, G., Dziewonski, A., Smith, G., and Su, W.-j. (1997). Elastic and Anelastic Structure Beneath Eurasia. Technical report, Harvard University.

Ekström, G. and Dziewonski, A. M. (1998). The unique anisotropy of the Pacific upper mantle. Nature, 394(6689):168.

Ekström, G., Nettles, M., and Dziewoński, A. (2012). The global CMT project 20042010: Centroid-moment tensors for 13,017 earthquakes. Physics of the Earth and Planetary Interiors, 200:1-9. 
Ellis, S., Van Dissen, R., Eberhart-Phillips, D., Reyners, M., Dolan, J., and Nicol, A. (2017). Detecting hazardous New Zealand faults at depth using seismic velocity gradients. Earth and Planetary Science Letters, 463:333-343.

Faccioli, E., Maggio, F., Quarteroni, A., and Taghan, A. (1996). Spectral-domain decomposition methods for the solution of acoustic and elastic wave equations. Geophysics, 61(4):1160-1174.

Fagereng, A. and Ellis, S. (2009). On factors controlling the depth of interseismic coupling on the Hikurangi subduction interface, New Zealand. Earth and Planetary Science Letters, 278(1-2):120-130.

Fichtner, A., Bunge, H.-P., and Igel, H. (2006a). The adjoint method in seismology: I. Theory. Physics of the Earth and Planetary Interiors, 157(1-2):86-104.

Fichtner, A., Bunge, H.-P., and Igel, H. (2006b). The adjoint method in seismology-: II. Applications: traveltimes and sensitivity functionals. Physics of the Earth and Planetary Interiors, 157(1-2):105-123.

Fichtner, A., Kennett, B. L., Igel, H., and Bunge, H.-P. (2009). Full seismic waveform tomography for upper-mantle structure in the Australasian region using adjoint methods. Geophysical Journal International, 179(3):1703-1725.

Fichtner, A., Kennett, B. L., Igel, H., and Bunge, H.-P. (2010). Full waveform tomography for radially anisotropic structure: new insights into present and past states of the Australasian upper mantle. Earth and Planetary Science Letters, 290(34):270-280.

Fichtner, A. and Leeuwen, T. v. (2015). Resolution analysis by random probing. Journal of Geophysical Research: Solid Earth, 120(8):5549-5573.

Fichtner, A. and Trampert, J. (2011a). Hessian kernels of seismic data functionals based upon adjoint techniques. Geophysical Journal International, 185(2):775-798.

Fichtner, A. and Trampert, J. (2011b). Resolution analysis in full waveform inversion. Geophysical Journal International, 187(3):1604-1624.

Fichtner, A., Trampert, J., Cupillard, P., Saygin, E., Taymaz, T., Capdeville, Y., and Villasenor, A. (2013). Multiscale full waveform inversion. Geophysical Journal International, 194(1):534-556. 
Fink, M. (1992). Time reversal of ultrasonic fields. I. Basic principles. IEEE transactions on Ultrasonics, Ferroelectrics, and Frequency Control, 39(5):555-566.

Fink, M. (1997). Time reversed acoustics. Physics Today, 50(3):34-40.

Fink, M., Prada, C., Wu, F., and Cassereau, D. (1989). Self focusing in inhomogeneous media with time reversal acoustic mirrors. In Proceedings., IEEE Ultrasonics Symposium, pages 681-686. IEEE.

Francis, D., Bennett, D., and Courteney, S. (2004). Advances in understanding of onshore East Coast Basin structure, stratigraphic thickness and hydrocarbon generation. In New Zealand Petroleum Conference Proceedings, pages 1-20.

Friedrich, A., Krüger, F., and Klinge, K. (1998). Ocean-generated microseismic noise located with the Gräfenberg array. Journal of Seismology, 2(1):47-64.

Fukao, Y., Widiyantoro, S., and Obayashi, M. (2001). Stagnant slabs in the upper and lower mantle transition region. Reviews of Geophysics, 39(3):291-323.

Gao, H. and Shen, Y. (2015). Validation of recent shear wave velocity models in the United States with full-wave simulation. J. Geophys. Res. Solid Earth, 120:344-358.

Gazdag, J. (1981). Modeling of the acoustic wave equation with transform methods. Geophysics, 46(6):854-859.

Gokhberg, A. and Fichtner, A. (2016). Full-waveform inversion on heterogeneous HPC systems. Computers E Geosciences, 89:260-268.

Graves, R., Jordan, T. H., Callaghan, S., Deelman, E., Field, E., Juve, G., Kesselman, C., Maechling, P., Mehta, G., Milner, K., et al. (2011). CyberShake: A physicsbased seismic hazard model for southern California. Pure and Applied Geophysics, 168(3):367-381.

Gung, Y., Panning, M., and Romanowicz, B. (2003). Global anisotropy and the thickness of continents. Nature, 422(6933):707-711.

Gutenberg, B. (1951). PKKP, $\mathrm{P}^{\prime} \mathrm{P}^{\prime}$, and the Earth's core. Eos, Transactions American Geophysical Union, 32(3):373-390.

Hamling, I. J., Hreinsdóttir, S., Clark, K., Elliott, J., Liang, C., Fielding, E., Litchfield, N., Villamor, P., Wallace, L., Wright, T. J., et al. (2017). Complex multifault 
rupture during the $2016 \mathrm{Mw} 7.8$ Kaikōura earthquake, New Zealand. Science, 356(6334):eaam7194.

Heise, W., Caldwell, T. G., Bannister, S., Bertrand, E., Ogawa, Y., Bennie, S., and Ichihara, H. (2017). Mapping subduction interface coupling using magnetotellurics: Hikurangi margin, New Zealand. Geophysical Research Letters, 44(18):9261-9266.

Heise, W., Caldwell, T. G., Bibby, H. M., and Bennie, S. L. (2010). Threedimensional electrical resistivity image of magma beneath an active continental rift, Taupo Volcanic Zone, New Zealand. Geophysical Research Letters, 37(10).

Henrys, S., Eberhart-Phillips, D., Bassett, D., Sutherland, R., Okaya, D., Savage, M., Evanzia, D., Stern, T., Sato, H., Mochizuki, K., et al. (2020). Upper plate heterogeneity along the southern Hikurangi Margin, New Zealand. Geophysical Research Letters, 47(4):e2019GL085511.

Henrys, S., Reyners, M., Pecher, I., Bannister, S., Nishimura, Y., and Maslen, G. (2006). Kinking of the subducting slab by escalator normal faulting beneath the North Island of New Zealand. Geology, 34(9):777-780.

Henrys, S., Wech, A., Sutherland, R., Stern, T., Savage, M., Sato, H., Mochizuki, K., Iwasaki, T., Okaya, D., Seward, A., et al. (2013). SAHKE geophysical transect reveals crustal and subduction zone structure at the southern Hikurangi margin, New Zealand. Geochemistry, Geophysics, Geosystems, 14(7):2063-2083.

Herman, G. T. (2009). Fundamentals of computerized tomography: image reconstruction from projections. Springer Science \& Business Media.

Hirose, H., Hirahara, K., Kimata, F., Fujii, N., and Miyazaki, S. (1999). A slow thrust slip event following the two 1996 Hyuganada earthquakes beneath the Bungo Channel, southwest Japan. Geophysical Research Letters, 26(21):3237-3240.

Holden, C., Kaneko, Y., D’Anastasio, E., Benites, R., Fry, B., and Hamling, I. (2017). The 2016 Kaikōura earthquake revealed by kinematic source inversion and seismic wavefield simulations: Slow rupture propagation on a geometrically complex crustal fault network. Geophysical Research Letters, 44(22):11-320. 
Holdgate, G. and Grapes, R. (2015). Wairau basin and fault connections across Cook strait, New Zealand: Seismic and geological evidence. Australian Journal of Earth Sciences, 62(1):95-121.

Hung, S.-H., Dahlen, F., and Nolet, G. (2001). Wavefront healing: a bananadoughnut perspective. Geophysical Journal International, 146(2):289-312.

Hunt, T. and Glover, R. (1995). Origin of mineral springs on the east coast, North Island, NZ. Technical report, Wairakei Research Centre, IGNS, Taupo, NZ.

Igel, H. (2017). Computational seismology: a practical introduction. Oxford University Press.

Jacobs, K., Savage, M., and Smith, E. (2016). Quantifying seismicity associated with slow slip events in the Hikurangi margin, New Zealand. New Zealand Journal of Geology and Geophysics, 59(1):58-69.

Julian, B., Gubbins, D., et al. (1977). Three-dimensional seismic ray tracing. Journal of Geophysics, 43(1):95-113.

Kaneko, Y., Ito, Y., Chow, B., Wallace, L. M., Tape, C., Grapenthin, R., D'Anastasio, E., Henrys, S., and Hino, R. (2019). Ultra-long Duration of Seismic Ground Motion Arising From a Thick, Low-Velocity Sedimentary Wedge. Journal of Geophysical Research: Solid Earth, 124(10):10347-10359.

Kaneko, Y., Wallace, L. M., Hamling, I. J., and Gerstenberger, M. C. (2018). Simple physical model for the probability of a subduction-zone earthquake following slow slip events and earthquakes: Application to the Hikurangi megathrust, New Zealand. Geophysical Research Letters, 45(9):3932-3941.

Kárason, H. and Van Der Hilst, R. D. (2000). Constraints on mantle convection from seismic tomography. Geophysical monograph, 121:277-288.

Kennett, B. (2009). Seismic wave propagation in stratified media. ANU Press.

Kim, Y., Liu, Q., and Tromp, J. (2011). Adjoint centroid-moment tensor inversions. Geophysical Journal International, 186(1):264-278.

King, P. R. and Thrasher, G. P. (1996). Cretaceous-Cenozoic geology and petroleum systems of the Taranaki Basin, New Zealand, volume 2. Institute of Geological \& Nuclear Sciences. 
King Hubbert, M. and Rubey, W. W. (1959). Role of fluid pressure in mechanics of overthrust faulting: I. Mechanics of fluid-filled porous solids and its application to overthrust faulting. Geological Society of America Bulletin, 70(2):115-166.

Komatitsch, D., Ritsema, J., and Tromp, J. (2002). The spectral-element method, Beowulf computing, and global seismology. Science, 298:1737-1742.

Komatitsch, D. and Tromp, J. (2002a). Spectral-element simulations of global seismic wave propagation-I. Validation. Geophysical Journal International, 149(2):390-412.

Komatitsch, D. and Tromp, J. (2002b). Spectral-element simulations of global seismic wave propagation-II. Three-dimensional models, oceans, rotation and selfgravitation. Geophysical Journal International, 150(1):303-318.

Komatitsch, D. and Vilotte, J.-P. (1998). The spectral element method: an efficient tool to simulate the seismic response of $2 \mathrm{D}$ and $3 \mathrm{D}$ geological structures. Bulletin of the Seismological Society of America, 88(2):368-392.

Koulakov, I. (2013). Studying deep sources of volcanism using multiscale seismic tomography. Journal of Volcanology and Geothermal Research, 257:205-226.

Krischer, L. (2015a). Pyadjoint. http:/ / krischer.github.io/pyadjoint/.

Krischer, L. (2015b). Pyflex. https: / /github.com/krischer/pyflex.

Krischer, L., Fichtner, A., Boehm, C., and Igel, H. (2018). Automated large-scale full seismic waveform inversion for North America and the North Atlantic. Journal of Geophysical Research: Solid Earth, 123(7):5902-5928.

Krischer, L., Fichtner, A., Zukauskaite, S., and Igel, H. (2015a). Large-scale seismic inversion framework. Seismological Research Letters, 86(4):1198-1207.

Krischer, L., Megies, T., Barsch, R., Beyreuther, M., Lecocq, T., Caudron, C., and Wassermann, J. (2015b). ObsPy: A bridge for seismology into the scientific Python ecosystem. Computational Science $\mathcal{E}$ Discovery, 8(1):014003.

Krischer, L., Smith, J., Lei, W., Lefebvre, M., Ruan, Y., de Andrade, E. S., Podhorszki, N., Bozdağ, E., and Tromp, J. (2016). An Adaptable Seismic Data Format. Geophysical Supplements to the Monthly Notices of the Royal Astronomical Society, 207(2):1003-1011. 
Lanza, F., Chamberlain, C., Jacobs, K., Warren-Smith, E., Godfrey, H., Kortink, M., Thurber, C., Savage, M., Townend, J., Roecker, S., et al. (2019). Crustal fault connectivity of the Mw 7.8 2016 Kaikōura earthquake constrained by aftershock relocations. Geophysical Research Letters, 46(12):6487-6496.

Lee, E.-J., Chen, P., and Jordan, T. H. (2014a). Testing waveform predictions of $3 \mathrm{D}$ velocity models against two recent Los Angeles earthquakes. Seismological Research Letters, 85(6):1275-1284.

Lee, E.-J., Chen, P., Jordan, T. H., Maechling, P. B., Denolle, M. A., and Beroza, G. C. (2014b). Full-3-D tomography for crustal structure in southern California based on the scattering-integral and the adjoint-wavefield methods. Journal of Geophysical Research: Solid Earth, 119(8):6421-6451.

Lee, E.-J., Chen, P., Jordan, T. H., Maechling, P. B., Denolle, M. A. M., and Beroza, G. C. (2014c). Full-3-D tomography for crustal structure in Southern California based on the scattering-integral and the adjoint-wavefield methods. J. Geophys. Res. Solid Earth, 119:6421-6451.

Leitner, B., Eberhart-Phillips, D., Anderson, H., and Nabelek, J. L. (2001). A focused look at the Alpine fault, New Zealand: Seismicity, focal mechanisms, and stress observations. Journal of Geophysical Research: Solid Earth, 106(B2):21932220.

Lewis, K. and Pettinga, J. (1993). The emerging, imbricate frontal wedge of the Hikurangi margin. Sedimentary Basins of the World, 2:225-250.

Lewis, K. B., Carter, L., and Davey, F. J. (1994). The opening of Cook Strait: interglacial tidal scour and aligning basins at a subduction to transform plate edge. Marine Geology, 116(3-4):293-312.

Lewis, K. B., Collot, J.-Y., and Lallem, S. E. (1998). The dammed Hikurangi Trough: a channel-fed trench blocked by subducting seamounts and their wake avalanches (New Zealand-France GeodyNZ Project). Basin Research, 10(4):441468.

Li, X.-D. and Romanowicz, B. (1996). Global mantle shear velocity model developed using nonlinear asymptotic coupling theory. Journal of Geophysical Research: Solid Earth, 101(B10):22245-22272. 
Lin, F.-C., Moschetti, M., and Ritzwoller, M. (2008). Surface wave tomography of the western United States from ambient seismic noise: Rayleigh and Love wave phase velocity maps. Geophysical Journal International, 173(1):281-298.

Lin, F.-C., Ritzwoller, M., Townend, J., Bannister, S., and Savage, M. (2007). Ambient noise Rayleigh wave tomography of New Zealand. Geophysical Journal International, 170(2):649-666.

Lin, Y.-P., Zhao, L., and Hung, S.-H. (2011). Assessment of tomography models of Taiwan using first-arrival times from the TAIGER active-source experiment. Bull. Seismol. Soc. Am., 101(2):866-880.

Litchfield, N., Ellis, S., Berryman, K., and Nicol, A. (2007). Insights into subduction-related uplift along the Hikurangi Margin, New Zealand, using numerical modeling. Journal of Geophysical Research: Earth Surface, 112(F2).

Litchfield, N., Van Dissen, R., Sutherland, R., Barnes, P., Cox, S., Norris, R., Beavan, R., Langridge, R., Villamor, P., Berryman, K., et al. (2014). A model of active faulting in New Zealand. New Zealand Journal of Geology and Geophysics, 57(1):3256.

Little, T. A. and Jones, A. (1998). Seven million years of strike-slip and related offfault deformation, northeastern Marlborough fault system, South Island, New Zealand. Tectonics, 17(2):285-302.

Little, T. A. and Roberts, A. P. (1997). Distribution and mechanism of Neogene to present-day vertical axis rotations, Pacific-Australian plate boundary zone, South Island, New Zealand. Journal of Geophysical Research: Solid Earth, 102(B9):20447-20468.

Liu, D. C. and Nocedal, J. (1989). On the limited memory BFGS method for large scale optimization. Mathematical programming, 45(1-3):503-528.

Liu, Q. and Gu, Y. (2012). Seismic imaging: From classical to adjoint tomography. Tectonophysics, 566:31-66.

Liu, Q. and Tromp, J. (2006). Finite-frequency kernels based on adjoint methods. Bulletin of the Seismological Society of America, 96(6):2383-2397. 
Lomax, A., Virieux, J., Volant, P., and Berge-Thierry, C. (2000). Probabilistic earthquake location in 3D and layered models. In Advances in seismic event location, pages 101-134. Springer.

Luo, Y. and Schuster, G. T. (1991). Wave-equation traveltime inversion. Geophysics, 56:645-653.

Maggi, A., Tape, C., Chen, M., Chao, D., and Tromp, J. (2009). An automated time-window selection algorithm for seismic tomography. Geophysical Journal International, 178(1):257-281.

Malcolm, A. E. and Trampert, J. (2011). Tomographic errors from wave front healing: more than just a fast bias. Geophysical Journal International, 185(1):385-402.

Marquering, H., Dahlen, F., and Nolet, G. (1999). Three-dimensional sensitivity kernels for finite-frequency traveltimes: the banana-doughnut paradox. Geophysical Journal International, 137(3):805-815.

Matharu, G. and Sacchi, M. D. (2018). Source encoding in multiparameter full waveform inversion. Geophys. J. Int., 214:792-810.

McNamara, D. E. and Boaz, R. (2006). Seismic noise analysis system using power spectral density probability density functions: A stand-alone software package. Citeseer.

McNamara, D. E. and Buland, R. P. (2004). Ambient noise levels in the continental United States. Bulletin of the Seismological Society of America, 94(4):1517-1527.

Menke, W. (2018). Geophysical data analysis: Discrete inverse theory. Academic press.

Métivier, L. and Brossier, R. (2016). The SEISCOPE optimization toolbox: A largescale nonlinear optimization library based on reverse communication. Geophysics, 81(2):F1-F15.

Miyoshi, T., Obayashi, M., Peter, D., Tono, Y., and Tsuboi, S. (2017). Adjoint tomography of the crust and upper mantle structure beneath the Kanto region using broadband seismograms. Progress in Earth and Planetary Science, 4(1):1-20.

Mochizuki, K., Sutherland, R., Henrys, S., Bassett, D., Van Avendonk, H., Arai, R., Kodaira, S., Fujie, G., Yamamoto, Y., Bangs, N., et al. (2019). Recycling of depleted continental mantle by subduction and plumes at the Hikurangi Plateau large igneous province, southwestern Pacific Ocean. Geology, 47(8):795-798. 
Modrak, R. and Tromp, J. (2016). Seismic waveform inversion best practices: regional, global and exploration test cases. Geophysical Journal International, 206(3):1864-1889.

Modrak, R. T., Borisov, D., Lefebvre, M., and Tromp, J. (2018). SeisFlows - Flexible waveform inversion software. Computers $\mathcal{E}$ Geosciences, 115:88-95.

Montelli, R., Nolet, G., Dahlen, F., and Masters, G. (2006). A catalogue of deep mantle plumes: New results from finite-frequency tomography. Geochemistry, Geophysics, Geosystems, 7(11).

Montelli, R., Nolet, G., Dahlen, F., Masters, G., Engdahl, E. R., and Hung, S.-H. (2004a). Finite-frequency tomography reveals a variety of plumes in the mantle. Science, 303(5656):338-343.

Montelli, R., Nolet, G., Masters, G., Dahlen, F., and Hung, S.-H. (2004b). Global P and PP traveltime tomography: rays versus waves. Geophysical Journal International, 158(2):637-654.

Moore, D. E. and Rymer, M. J. (2007). Talc-bearing serpentinite and the creeping section of the San Andreas fault. Nature, 448(7155):795.

Mortimer, N. (2004). New Zealand's geological foundations. Gondwana research, 7(1):261-272.

Mortimer, N. and Parkinson, D. (1996). Hikurangi Plateau: A Cretaceous large igneous province in the southwest Pacific Ocean. Journal of Geophysical Research: Solid Earth, 101(B1):687-696.

Nazarian, S. and Stokoe, K. H. (1984). Nondestructive testing of pavements using surface waves. Transportation Research Record, 993:67-79.

Neall, V. E., Smith, I. E., and Stewart, R. (1986). History and petrology of the Taranaki volcanoes. Royal Society of New Zealand.

Nicol, A. and Beavan, J. (2003). Shortening of an overriding plate and its implications for slip on a subduction thrust, central Hikurangi Margin, New Zealand. Tectonics, 22(6). 
Nicol, A., Mazengarb, C., Chanier, F., Rait, G., Uruski, C., and Wallace, L. (2007). Tectonic evolution of the active Hikurangi subduction margin, New Zealand, since the Oligocene. Tectonics, 26(4).

Nolet, G. (1987). Seismic wave propagation and seismic tomography. In Seismic tomography, pages 1-23. Springer.

Nolet, G. (2008). A breviary of seismic tomography. A Breviary of Seismic Tomography.

Nolet, G. and Dahlen, F. (2000). Wave front healing and the evolution of seismic delay times. Journal of Geophysical Research: Solid Earth, 105(B8):19043-19054.

Norris, R. J., Cooper, A. F., Okaya, D., Stern, T., and Davey, F. (2007). The Alpine Fault, New Zealand: surface geology and field relationships. Geophysical Monograph-American Geophysical Union, 175:159.

Ohta, Y., Freymueller, J. T., Hreinsdóttir, S., and Suito, H. (2006). A large slow slip event and the depth of the seismogenic zone in the south central Alaska subduction zone. Earth and Planetary Science Letters, 247(1-2):108-116.

Pandas Development Team (2020). pandas-dev/pandas: Pandas.

Patera, A. T. (1984). A spectral element method for fluid dynamics: laminar flow in a channel expansion. Journal of Computational Physics, 54(3):468-488.

Pedley, K. L., Barnes, P. M., Pettinga, J. R., and Lewis, K. B. (2010). Seafloor structural geomorphic evolution of the accretionary frontal wedge in response to seamount subduction, Poverty Indentation, New Zealand. Marine Geology, 270(1-4):119-138.

Plessix, R.-E. (2006). A review of the adjoint-state method for computing the gradient of a functional with geophysical applications. Geophysical Journal International, 167(2):495-503.

Pondard, N. and Barnes, P. M. (2010). Structure and paleoearthquake records of active submarine faults, Cook Strait, New Zealand: Implications for fault interactions, stress loading, and seismic hazard. Journal of Geophysical Research: Solid Earth, 115(B12). 
Price, R., Stewart, R., Woodhead, J., and Smith, I. (1999). Petrogenesis of high-K arc magmas: evidence from Egmont volcano, North Island, New Zealand. Journal of Petrology, 40(1):167-197.

Priolo, E., Carcione, J. M., and Seriani, G. (1994). Numerical simulation of interface waves by high-order spectral modeling techniques. The Journal of the Acoustical Society of America, 95(2):681-693.

Radiguet, M., Cotton, F., Vergnolle, M., Campillo, M., Walpersdorf, A., Cotte, N., and Kostoglodov, V. (2012). Slow slip events and strain accumulation in the Guerrero gap, Mexico. Journal of Geophysical Research: Solid Earth, 117(B4).

Rawlinson, N., Pilia, S., Young, M., Salmon, M., and Yang, Y. (2016). Crust and upper mantle structure beneath southeast Australia from ambient noise and teleseismic tomography. Tectonophysics, 689:143-156.

Reyes, A., Christenson, B., and Faure, K. (2010). Sources of solutes and heat in low-enthalpy mineral waters and their relation to tectonic setting, New Zealand. Journal of Volcanology and Geothermal Research, 192(3-4):117-141.

Reyners, M. (1998). Plate coupling and the hazard of large subduction thrust earthquakes at the Hikurangi subduction zone, New Zealand. New Zealand Journal of Geology and Geophysics, 41(4):343-354.

Reyners, M., Eberhart-Phillips, D., and Bannister, S. (2011). Tracking repeated subduction of the Hikurangi Plateau beneath New Zealand. Earth and Planetary Science Letters, 311(1-2):165-171.

Reyners, M., Eberhart-Phillips, D., and Bannister, S. (2017). Subducting an old subduction zone sideways provides insights into what controls plate coupling. Earth and Planetary Science Letters, 466:53-61.

Reyners, M., Eberhart-Phillips, D., Stuart, G., and Nishimura, Y. (2006). Imaging subduction from the trench to $300 \mathrm{~km}$ depth beneath the central North Island, New Zealand, with Vp and Vp/Vs. Geophysical Journal International, 165(2):565583.

Ristau, J. (2008). Implementation of routine regional moment tensor analysis in New Zealand. Seismol. Res. Lett., 79(3):400-415. 
Ristau, J. (2013). Update of regional moment tensor analysis for earthquakes in New Zealand and adjacent offshore regions. Bulletin of the Seismological Society of America, 103(4):2520-2533.

Ritsema, J., van Heijst, H. J., and Woodhouse, J. H. (1999). Complex shear wave velocity structure imaged beneath Africa and Iceland. Science, 286(5446):19251928.

Robinson, R. and Webb, T. H. (1996). AMPRAT and MECHTOOL: programs for determining focal mechanisms of local earthquakes, volume 96. Institute of Geological \& Nuclear Sciences Ltd.

Romanowicz, B. (1991). Seismic tomography of the Earth's mantle. Annual Review of Earth and Planetary Sciences, 19(1):77-99.

Rowland, J. V., Wilson, C. J., and Gravley, D. M. (2010). Spatial and temporal variations in magma-assisted rifting, Taupō Volcanic Zone, New Zealand. Journal of Volcanology and Geothermal Research, 190(1):89-108.

Ruan, Y., Lei, W., Modrak, R., Örsvuran, R., Bozdağ, E., and Tromp, J. (2019). Balancing unevenly distributed data in seismic tomography: a global adjoint tomography example. Geophysical Journal International, 219(2):1225-1236.

Rusmanugroho, H., Modrak, R., and Tromp, J. (2017). Anisotropic full-waveform inversion with tilt-angle recovery. Geophysics, 82(3):1-17.

Seriani, G. z. and Priolo, E. (1994). Spectral element method for acoustic wave simulation in heterogeneous media. Finite Elements in Analysis and Design, 16(3):337348.

Shannon, C. E. (1949). Communication in the presence of noise. Proceedings of the IRE, 37(1):10-21.

Shapiro, N., Campillo, M., Stehly, L., and Ritzwoller, M. (2005). High-resolution surface-wave tomography from ambient seismic noise. Science, 307(5715):16151618.

Sherburn, S. and White, R. S. (2006). Tectonics of the Taranaki region, New Zealand: earthquake focal mechanisms and stress axes. New Zealand Journal of Geology and Geophysics, 49(2):269-279. 
Sherburn, S., White, R. S., and Chadwick, M. (2006). Three-dimensional tomographic imaging of the Taranaki volcanoes, New Zealand. Geophysical Journal International, 166(2):957-969.

Sibson, R. H. (1982). Fault zone models, heat flow, and the depth distribution of earthquakes in the continental crust of the United States. Bulletin of the Seismological Society of America, 72(1):151-163.

Simons, F. J. and van der Hilst, R. D. (2003). Seismic and mechanical anisotropy and the past and present deformation of the Australian lithosphere. Earth and Planetary Science Letters, 211(3-4):271-286.

Smith, J. A., Borisov, D., Cudney, H., Miller, R. D., Modrak, R., Moran, M., Peterie, S. L., Sloan, S. D., Tromp, J., and Wang, Y. (2019). Tunnel detection at Yuma Proving Ground, Arizona, USA-Part 2: 3D full-waveform inversion experiments. Geophysics, 84(1):B95-B108.

Snieder, R., Wapenaar, K., et al. (2010). Imaging with ambient noise. Physics Today, 63(9):44-49.

Stagpoole, V., Miller, C., Caratori Tontini, F., Brakenrig, T., and Macdonald, N. (2020). A two million-year history of rifting and caldera volcanism imprinted in new gravity anomaly compilation of the Taupō Volcanic Zone, New Zealand. New Zealand Journal of Geology and Geophysics, pages 1-14.

Stein, S. and Wysession, M. (2009). An introduction to seismology, earthquakes, and earth structure. John Wiley \& Sons.

Stern, T. (1985). A back-arc basin formed within continental lithosphere: the Central Volcanic Region of New Zealand. Tectonophysics, 112(1-4):385-409.

Stirling, M., McVerry, G., Gerstenberger, M., Litchfield, N., Van Dissen, R., Berryman, K., Barnes, P., Wallace, L., Villamor, P., Langridge, R., Lamarche, G., Nodder, S., Reyners, M., Bradley, B., Rhoades, D., Smith, W., Nicol, A., Pettinga, J., Clark, K., and Jacobs, K. (2012). National seismic hazard model for New Zealand: 2010 update. Bulletin of the Seismological Society of America, 102(4):15141542.

Su, W.-j., Woodward, R. L., and Dziewonski, A. M. (1992). Deep origin of midocean-ridge seismic velocity anomalies. Nature, 360(6400):149-152. 
Sun, T., Saffer, D., and Ellis, S. (2020). Mechanical and hydrological effects of seamount subduction on megathrust stress and slip. Nature Geoscience, 13(3):249-255.

Sutherland, R., Eberhart-Phillips, D., Harris, R., Stern, T., Beavan, J., Ellis, S., Henrys, S., Cox, S., Norris, R., Berryman, K., et al. (2007). Do great earthquakes occur on the Alpine fault in central South Island, New Zealand? Geophysical Monagraph - American Geophysical Union, 175:237.

Taborda, R., Azizzadeh-Roodpish, S., Khoshnevis, N., and Cheng, K. (2016). Evaluation of the southern California seismic velocity models through simulation of recorded events. Geophysical Journal International, 205:1342-1364.

Talagrand, O. and Courtier, P. (1987). Variational assimilation of meteorological observations with the adjoint vorticity equation. I: Theory. Quarterly Journal of the Royal Meteorological Society, 113(478):1311-1328.

Tao, K., Grand, S. P., and Niu, F. (2018). Seismic structure of the upper mantle beneath eastern Asia from full waveform seismic tomography. Geochemistry, Geophysics, Geosystems, 19(8):2732-2763.

Tape, C., Liu, Q., Maggi, A., and Tromp, J. (2010). Seismic tomography of the southern California crust based on spectral-element and adjoint methods. Geophysical Journal International, 180(1):433-462.

Tape, C., Liu, Q., and Tromp, J. (2007). Finite-frequency tomography using adjoint methods-Methodology and examples using membrane surface waves. Geophysical Journal International, 168(3):1105-1129.

Tape, C. H. (2009). Seismic tomography of southern California using adjoint methods. $\mathrm{PhD}$ thesis, California Institute of Technology.

Tarantola, A. (1984). Inversion of seismic reflection data in the acoustic approximation. Geophysics, 49(8):1259-1266.

Tarantola, A. (2005). Inverse problem theory and methods for model parameter estimation, volume 89. Siam.

Taylor, B. (2006). The single largest oceanic plateau: Ontong Java-ManihikiHikurangi. Earth and Planetary Science Letters, 241(3-4):372-380. 
Thrastarson, S., van Driel, M., Krischer, L., Boehm, C., Afanasiev, M., Van Herwaarden, D.-P., and Fichtner, A. (2020). Accelerating numerical wave propagation by wavefield adapted meshes. Part II: full-waveform inversion. Geophysical Journal International, 221(3):1591-1604.

Thurber, C. (1993). Local earthquake tomography: velocities and V_p/V_sTheory. Seismic tomography: theory and practice, pages 563-583.

Townend, J., Sherburn, S., Arnold, R., Boese, C., and Woods, L. (2012). Threedimensional variations in present-day tectonic stress along the Australia-Pacific plate boundary in New Zealand. Earth and Planetary Science Letters, 353:47-59.

Tromp, J. and Bachmann, E. (2019a). Source encoding for adjoint tomography. Geophysical Journal International, 218(3):2019-2044.

Tromp, J. and Bachmann, E. (2019b). Source encoding for adjoint tomography. Geophysical Journal International, 218:2019-2044.

Tromp, J., Tape, C., and Liu, Q. (2005). Seismic tomography, adjoint methods, time reversal and banana-doughnut kernels. Geophysical Journal International, 160(1):195-216.

Uruski, C. (1992). Sedimentary Basins \& Structure of Cook Strait, volume 92. Institute of Geological and Nuclear Sciences Limited.

Van Der Hilst, R. D. and De Hoop, M. V. (2005). Banana-'-doughnut kernels and mantle tomography. Geophysical Journal International, 163(3):956-961.

van der Hilst, R. D. and Kárason, H. (1999). Compositional heterogeneity in the bottom 1000 kilometers of Earth's mantle: Toward a hybrid convection model. Science, 283(5409):1885-1888.

Van Dissen, R. J. and Berryman, K. R. (1996). Surface rupture earthquakes over the last 1000 years in the Wellington region, New Zealand, and implications for ground shaking hazard. Journal of Geophysical Research: Solid Earth, 101(B3):5999_ 6019.

van Driel, M., Boehm, C., Krischer, L., and Afanasiev, M. (2020). Accelerating numerical wave propagation using wavefield adapted meshes. Part I: forward and adjoint modelling. Geophysical Journal International, 221(3):1580-1590. 
van Herwaarden, D. P., Boehm, C., Afanasiev, M., Thrastarson, S., Krischer, L., Trampert, J., and Fichtner, A. (2020). Accelerated full-waveform inversion using dynamic mini-batches. Geophysical Journal International, 221(2):1427-1438.

Villamor, P. and Berryman, K. (2006). Evolution of the southern termination of the Taupō Rift, New Zealand. New Zealand Journal of Geology and Geophysics, 49(1):23-37.

Villamor, P., Berryman, K., Ellis, S., Schreurs, G., Wallace, L., Leonard, G., Langridge, R., and Ries, W. (2017). Rapid evolution of subduction-related continental intraarc rifts: The Taupo Rift, New Zealand. Tectonics, 36(10):2250-2272.

Walcott, R. (1978). Present tectonics and late Cenozoic evolution of New Zealand. Geophysical Journal International, 52(1):137-164.

Wallace, L., Barnes, P., Beavan, J., Van Dissen, R., Litchfield, N., Mountjoy, J., Langridge, R., Lamarche, G., and Pondard, N. (2012a). The kinematics of a transition from subduction to strike-slip: An example from the central New Zealand plate boundary. Journal of Geophysical Research: Solid Earth, 117(B2).

Wallace, L., Reyners, M., Cochran, U., Bannister, S., Barnes, P., Berryman, K., Downes, G., Eberhart-Phillips, D., Fagereng, A., Ellis, S., Nicol, A., McCaffrey, R., Beavan, R., Henrys, S., Sutherland, R., Barker, D., Litchfield, N., Townend, J., Robinson, R., Bell, R., Wilson, K., and Power, W. (2009). Characterizing the seismogenic zone of a major plate boundary subduction thrust: Hikurangi Margin, New Zealand. Geochemistry, Geophysics, Geosystems, 10(10).

Wallace, L. M. (2020). Slow slip events in New Zealand. Annual Review of Earth and Planetary Sciences, 48:175-203.

Wallace, L. M. and Beavan, J. (2006). A large slow slip event on the central Hikurangi subduction interface beneath the Manawatu region, North Island, New Zealand. Geophysical Research Letters, 33(11).

Wallace, L. M. and Beavan, J. (2010). Diverse slow slip behavior at the Hikurangi subduction margin, New Zealand. Journal of Geophysical Research: Solid Earth, 115(B12).

Wallace, L. M., Beavan, J., Bannister, S., and Williams, C. (2012b). Simultaneous long-term and short-term slow slip events at the Hikurangi subduction margin, 
New Zealand: Implications for processes that control slow slip event occurrence, duration, and migration. Journal of Geophysical Research: Solid Earth, 117(B11).

Wallace, L. M., Beavan, J., McCaffrey, R., Berryman, K., and Denys, P. (2007). Balancing the plate motion budget in the South Island, New Zealand using GPS, geological and seismological data. Geophysical Journal International, 168(1):332352.

Wallace, L. M., Beavan, J., McCaffrey, R., and Darby, D. (2004). Subduction zone coupling and tectonic block rotations in the North Island, New Zealand. Journal of Geophysical Research: Solid Earth, 109(B12).

Wallace, L. M., Cochran, U. A., Power, W. L., and Clark, K. J. (2014). Earthquake and tsunami potential of the Hikurangi subduction thrust, New Zealand: Insights from paleoseismology, GPS, and tsunami modeling. Oceanography, 27(2):104-117.

Warren-Smith, E., Fry, B., Wallace, L., Chon, E., Henrys, S., Sheehan, A., Mochizuki, K., Schwartz, S., Webb, S., and Lebedev, S. (2019). Episodic stress and fluid pressure cycling in subducting oceanic crust during slow slip. Nature Geoscience, 12(6):475-481.

Webb, S. C. (1998). Broadband seismology and noise under the ocean. Reviews of Geophysics, 36(1):105-142.

Wes McKinney (2010). Data Structures for Statistical Computing in Python. In Stéfan van der Walt and Jarrod Millman, editors, Proceedings of the 9th Python in Science Conference, pages 56-61.

Wilks, M., Rawlinson, N., Kendall, J.-M., Nowacki, A., Biggs, J., Ayele, A., and Wookey, J. (2020). The coupled magmatic and hydrothermal systems of the restless Aluto caldera, Ethiopia.

Williams, C. and Wallace, L. (2018). The impact of realistic elastic properties on inversions of shallow subduction interface slow slip events using seafloor geodetic data. Geophysical Research Letters, 45(15):7462-7470.

Williams, C. A., Eberhart-Phillips, D., Bannister, S., Barker, D. H., Henrys, S., Reyners, M., and Sutherland, R. (2013). Revised interface geometry for the Hikurangi subduction zone, New Zealand. Seismological Research Letters, 84(6):1066-1073. 
Wilson, C., Gravley, D., Leonard, G., and Rowland, J. (2009). Volcanism in the central Taupo Volcanic Zone, New Zealand: tempo, styles and controls. Studies in Volcanology: the Legacy of George Walker. Special Publications of IAVCEI, 2:225247.

Wilson, C., Houghton, B., McWilliams, M., Lanphere, M., Weaver, S., and Briggs, R. (1995). Volcanic and structural evolution of Taupo Volcanic Zone, New Zealand: a review. Journal of Volcanology and Geothermal Research, 68(1-3):1-28.

Wilson, C. J. and Rowland, J. V. (2016). The volcanic, magmatic and tectonic setting of the Taupo Volcanic Zone, New Zealand, reviewed from a geothermal perspective. Geothermics, 59:168-187.

Wood, R. and Davy, B. (1994). The Hikurangi Plateau. Marine Geology, 118(12):153-173.

Woodhouse, J. H. and Dziewonski, A. M. (1984). Mapping the upper mantle: Three-dimensional modeling of Earth structure by inversion of seismic waveforms. Journal of Geophysical Research: Solid Earth, 89(B7):5953-5986.

Yao, H., Van Der Hilst, R., and Montagner, J.-P. (2010). Heterogeneity and anisotropy of the lithosphere of SE Tibet from surface wave array tomography. Journal of Geophysical Research: Solid Earth, 115(12).

Zhang, Y.-S. and Tanimoto, T. (1993). High-resolution global upper mantle structure and plate tectonics. Journal of Geophysical Research: Solid Earth, 98(B6):97939823.

Zhao, L., Jordan, T. H., and Chapman, C. H. (2000). Three-dimensional Fréchet differential kernels for seismicdelay times. Geophysical Journal International, 141(3):558-576.

Zhu, H., Bozdağ, E., and Tromp, J. (2015). Seismic structure of the European upper mantle based on adjoint tomography. Geophysical Journal International, 201(1):1852.

Zienkiewicz, O. and Taylor, R. (1989). The finite element method: Basic formulation and linear problems, Vol. I. Maidenhead: McGraw-Hill. 